\title{
A Mechanistic Approach to Tactile Friction
}

Julien van Kuilenburg 

A Mechanistic Approach to Tactile Friction

Julien van Kuilenburg 
Part of this research was carried out under project numbers MA.08113 and M63.2.10409 in the framework of the Research Program of the Materials innovation institute M2i (www.m2i.nl).

De promotiecommissie is als volgt opgesteld:

prof. dr. G.P.M.R. Dewulf, Universiteit Twente, voorzitter en secretaris prof. dr. ir. E. van der Heide, Universiteit Twente, promotor dr. ir. M.A. Masen, Imperial College London / Universiteit Twente, assistent-promotor

prof. dr. R. Lewis, University of Sheffield, UK

prof. dr. U. Olofsson, KTH Royal Institute of Technology, Sweden

prof. dr. ir. A. de Boer, Universiteit Twente

prof. dr. ir. A.J. Huis in 't Veld, Universiteit Twente

prof. dr. ir. H.F.J.M. Koopman, Universiteit Twente

Kuilenburg, Julien van

A Mechanistic Approach to Tactile Friction

PhD Thesis, University of Twente, Enschede, The Netherlands,

October 2013

ISBN 978-90-365-0034-0

Keywords: tribology, skin, friction, contact modelling, surface textures

Copyright (C)2013, J. van Kuilenburg, Eindhoven, The Netherlands

Printed by Wöhrmann Print Service, Zutphen, The Netherlands 


\section{A MECHANISTIC APPROACH TO TACTILE FRICTION}

\section{PROEFSCHRIFT}

ter verkrijging van

de graad van doctor aan de Universiteit Twente, op gezag van de rector magnificus, prof. dr. H. Brinksma, volgens besluit van het College voor Promoties in het openbaar te verdedigen op woensdag 23 oktober 2013 om 14:45 uur

door

Julien van Kuilenburg

geboren op 12 september 1974

te Tiel 
Dit proefschrift is goedgekeurd door:

de promotor prof. dr. ir. E. van der Heide de assistent-promotor dr. ir. M.A. Masen 
voor Maaike en Igor 

Part I 



\section{Summary}

To a large extent, the functionality and comfort experienced during the use of everyday products, such as apparel, household appliances and sports equipment, are determined by the frictional behaviour of contact that occurs with the skin. For product engineers who aim to control and optimize the sensorial properties of a product surface interacting with the skin, it is essential to understand this frictional behaviour. This involves the study of local friction behaviour at the scale of the surface roughness.

In this work a mechanistic approach was adopted in which analytical models from contact mechanics theory were used to develop a model which describes the tactile friction behaviour against the human fingerpad as a function of asperity geometry and operational conditions.

A model system of a rigid sphere sliding against the skin of the volar forearm was used to investigate friction theoretically. To account for the multilayered and nonhomogeneous structure of the skin the concept of an effective elastic modulus $E=f(a)$ was adopted, providing a closed-form expression describing the elasticity of the skin of the volar forearm as a function of length scale $a$. The effective elastic modulus decreases several orders of magnitude with increasing length scale, from about $1 \mathrm{MPa}$ at a length scale of $1 \mu \mathrm{m}$ to several $\mathrm{kPa}$ at the millimeter scale. The distinctive surface topography of the skin is referred to as the skin microrelief; at the volar forearm a pattern of plateaus and valleys. A multiscale model was developed in this work to take into account the influence of the skin microrelief. Incorporation of the contact model into a two-term friction model enables the prediction of the friction behaviour. 
Friction in contacts where the human fingerpad is one of the interacting surfaces, which is referred to as 'tactile friction', was described as analogous to the smooth spherical probe in contact with the skin by considering the fingerpad as a deformable sphere. The fingerprint ridges were modelled as annulus-shaped line contacts, characterized by a radius and contact width. The model developed in this thesis estimates the friction behaviour as a function of asperity geometry and operational conditions. To investigate the friction behaviour at the asperity level, surface textures consisting of evenly distributed spherically-tipped asperities were produced using ultra-short pulsed laser technology. The resulting friction force is calculated from the real area of contact and the indentation of the spherical asperities.

Sliding friction was measured in vivo between textured samples and the skin of the human fingerpad. The model gives insight into the mechanisms which determine the measured coefficient of friction. The results showed that the friction behaviour one observes when exploring a surface by touching is susceptible to different sources of variation, which are intrinsic to tactile exploration. The variation in observed results is the largest under conditions where normal adhesion plays an important role, i.e. the actual contact area with the skin is large. Therefore, a well-defined experimental procedure should be followed when carrying out a tactile friction experiment. 


\section{Samenvatting}

Functionaliteit en comfort tijdens het gebruik van dagelijkse producten, zoals kleding, huishoudelijke apparaten en sportproducten, worden voor een groot deel bepaald door het wrijvingsgedrag in het contact met de huid. Voor de productontwikkelaars, die de sensorische eigenschappen van een productoppervlak in contact met huid willen optimaliseren, is het van groot belang dat zij dit wrijvingsgedrag begrijpen. Dit brengt het onderzoek naar het lokale wrijvingsgedrag op het niveau van oppervlakteruwheid met zich mee.

In dit proefschrift is een mechanistische benadering gehanteerd, waarbij analytische modellen uit de contactmechanica zijn gebruikt om een model te ontwikkelen dat het wrijvingsgedrag tegen de vingertop beschrijft als functie van ruwheidsgeometrie en operationele condities.

Een modelsysteem van een bol glijdend over de huid van de onderarm is gebruikt voor een theoretisch onderzoek van huidwrijving. Rekening houdend met de gelaagde en inhomogene structuur van de huid, is gebruik gemaakt van het idee van een effectieve elasticiteitsmodulus $E=f(a)$, een gesloten vergelijking die de elasticiteit van de huid beschrijft als functie van de lengteschaal $a$. De effective elasticiteitsmodulus neemt enkele ordes in grootte af met toenemende lengteschaal, van ongeveer $1 \mathrm{MPa}$ op een lengteschaal van $1 \mathrm{~mm}$ tot enkele $\mathrm{kPa}$ 's op een schaal van millimeters. De karakteristieke oppervlakte topografie van de huid bestaat op de onderarm uit een patroon van plateaus en groeven. Om de invloed van de oppervlaktetopografie van de huid mee te nemen in het model is een 'multiscale' model ontwikkeld. Samenvoeging van het contact model met het wrijvingsmodel maakt het mogelijk het wrijvingsgedrag te voorspellen. 
Wrijving in contacten waarin de vingertop een van de contactoppervlakken is, wordt 'tactiele wrijving' genoemd. Het contact tussen de vinger en een nominaal vlak, hard oppervlak is beschreven analoog aan de situatie van de gladde bol in contact met de huid door de vingertop te beschouwen als een vervormbare bol. De oppervlaktetopografie van de vingertop is gemodelleerd als een combinatie van ringvormige lijncontacten, gekarakteriseerd door een radius en contactbreedte. Het ontwikkelde model berekent de wrijvingskracht als functie van de ruwheidsgeometrie en operationele condities. Voor het onderzoek van het wrijvingsgedrag op het niveau van een oppervlakteruwheid zijn er oppervlaketexturen geproduceerd door middel van 'ultra-short pulsed laser' technologie. De resulterende wrijvingskracht is berekend uit het ware contactoppervlak en de indringing van de bolvormige ruwheidstoppen.

Wrijving is in vivo gemeten tussen getextureerde oppervlakken en de huid van de vingertop. Het model verschaft inzicht in de mechanismen die verantwoordelijk zijn voor de gemeten wrijvingscoëfficiënt. De resultaten laten zien dat de wrijving die men waarneemt wanneer men een oppervlak tactiel onderzoekt onderhevig is aan verschillende bronnen van variatie, welke inherent zijn aan tast. De spreiding in de gemeten resultaten is het grootst wanneer adhesie tussen de oppervlakken een rol speelt, met andere woorden, als het werkelijke contactoppervlak met de huid groot is. Daarom moet bij het meten van tactiele wrijving een welomschreven experimentele procedure worden gevolgd. 


\section{Contents}

Summary I

Samenvatting III

Contents

$\begin{array}{lll}\text { Nomenclature } & \text { VII }\end{array}$

1 Introduction 1

1.1 Tactile perception . . . . . . . . . . . . . . 1

1.2 Skin tribology . . . . . . . . . . . . . . . . . . . . . . . . . . . . . . . . .

1.3 Tactile friction . . . . . . . . . . . . . . 6

1.4 Objectives of this research . . . . . . . . . . . 7

1.5 This thesis ..................... 8

2 Contact and Friction of Skin 10

2.1 Two-term friction model . . . . . . . . . . . . . . . . 10

2.2 Contact between a sphere and the skin . . . . . . . . . . 13

2.2.1 Length scale effect . . . . . . . . . . . . . . . . . . . . . . . . . . 13

2.2 .2 Skin microrelief . . . . . . . . . . . . . . . . 18

2.2.3 Adhesion. . . . . . . . . . . . . . . 21

2.3 Friction between a sphere and the skin . . . . . . . . . . 22

3 Modelling Tactile Friction 25

3.1 Review of fingerpad friction . . . . . . . . . . . . . 25

3.2 Contact between the fingerpad and a textured surface . . . . . 26

3.2.1 Fingerprint area . . . . . . . . . . . . . 27

3.2.2 Regular surface textures . . . . . . . . . . . 29 
3.3 Friction between the fingerpad and a textured surface . . . . . 31

4 Measuring Tactile Friction 33

4.1 Experimental methods . . . . . . . . . . . . . . . 33

4.1.1 Surface textures . . . . . . . . . . . . . 33

4.1.2 Friction measurement . . . . . . . . . . . . . . 35

4.1 .3 In vivo experiments . . . . . . . . . . . . . . . . . . . . . . . . . . . . .

4.2 Experiments using textured surfaces . . . . . . . . . . . . . 41

4.3 Experiments using sandpaper . . . . . . . . . . . . . 45

5 Conclusions and Recommendations $\quad 47$

5.1 Conclusions . . . . . . . . . . . . . . . . . . . . 47

5.2 Recommendations for future research . . . . . . . . . . . . 49

$\begin{array}{ll}\text { Bibliography } & 57\end{array}$ 


\section{Nomenclature}

\section{Roman symbols}

$\begin{array}{lll}a & \text { contact radius } & {[\mathrm{m}]} \\ A & \text { contact area } & {\left[\mathrm{m}^{2}\right]} \\ A_{0} & \text { apparent contact area } & {\left[\mathrm{m}^{2}\right]} \\ A_{1} & \text { skin contact area } & {\left[\mathrm{m}^{2}\right]} \\ A_{\text {real }} & \text { real contact area } & {\left[\mathrm{m}^{2}\right]} \\ C & \text { model parameter } & \\ d & \text { displacement } & {[\mathrm{m}]} \\ D & \text { diameter } & {[\mathrm{m}]} \\ E_{i} & \text { elastic modulus of body } i & {[\mathrm{~Pa}]} \\ E^{*} & \text { reduced elastic modulus } & {[\mathrm{Pa}]} \\ E_{e f f}^{*} & \text { effective elastic modulus } & {[\mathrm{Pa}]} \\ F & \text { normal load } & {[\mathrm{N}]} \\ F_{a d h} & \text { adhesion force } & {[\mathrm{N}]} \\ F_{a p p} & \text { apparent normal load } & {[\mathrm{N}]} \\ F_{\mu} & \text { friction force } & {[\mathrm{N}]} \\ h & \text { height } & {[\mathrm{m}]} \\ K & \text { model parameter } & \\ l & \text { contact length } & {[\mathrm{m}]} \\ n & \text { number of repeats } & {[-]} \\ N & \text { number of asperity contacts } & {[-]} \\ p & \text { pressure } & {[\mathrm{Pa}]} \\ Q & \text { heat flux } & {\left[\mathrm{J} / \mathrm{m}^{2} \mathrm{~s}\right]} \\ R & \text { (tip) radius of curvature } & {[\mathrm{m}]} \\ R_{a} & \text { centre-line-average roughness } & {[\mathrm{m}]} \\ t_{i} & \text { thickness of } i \text { th layer } & {[\mathrm{m}]} \\ v & \text { sliding velocity } & {[\mathrm{m} / \mathrm{s}]} \\ W_{12} & \text { work of adhesion } & {[\mathrm{N} / \mathrm{m}]}\end{array}$




\section{Greek symbols}

$\begin{array}{lll}\beta & \text { viscoelastic loss fraction } & {[-]} \\ \gamma_{i} & \text { surface free energy of material } i & {[\mathrm{~N} / \mathrm{m}]} \\ \delta & \text { indentation, deformation } & {[\mathrm{m}]} \\ \varphi & \text { angle } & {[-]} \\ \eta & \text { (areal) density of surface features } & {[/ \mathrm{m}],\left[/ \mathrm{m}^{2}\right]} \\ \lambda & \text { spacing } & {[\mathrm{m}]} \\ \mu & \text { coefficient of friction } & {[-]} \\ \nu & \text { Poisson's ratio } & {[-]} \\ \tau & \text { shear strength of the interface } & {[\mathrm{Pa}]} \\ \tau & \text { subsurface shear stress } & {[\mathrm{Pa}]}\end{array}$

\section{Indices}

adh due to adhesion

def due to deformation

$\mathrm{H}$ calculated using Hertz's theory

JKR calculated using JKR theory 


\section{Chapter 1}

\section{Introduction}

\subsection{Tactile perception}

Every day from the moment we wake up, and even before, we touch, hold and wear a wide range of products. From the personal care products in our bathroom, the cutlery we use during breakfast to the machinery we operate at work, the frictional behaviour of contact that occurs with the skin determines to a large extent the functionality and comfort during use of these products. For example; in consumer electronics, the rotating knobs to control sound volume and balance require high friction to provide grip and, in combination with a resistance torque, a certain amount of force feedback. In handheld devices, where those knobs are eliminated, touchscreens require low friction to enable the fingerpad to slide comfortably and to allow precise operation. Not only functional performance, such as grip or sliding behaviour, but also the touch properties of the product surface are influenced by the friction behaviour. These properties, together with the other sensorial properties sight, smell, taste and hearing, create a sensorial experience which determines the impression of a product [1]. The objective properties of products and materials, such as the frictional properties, which are perceived by our senses, will generate subjective impressions.

Analysis of the tactile qualities described from a tribological point of view shows that in the sliding contact between the fingerpad and a rough surface, friction plays a role in the perception of warmth, roughness and slipperiness. The correlation with friction may be direct or indirect. In a 
rubbing contact, the contact temperature will increase proportional to the coefficient of friction as described by the theory of contact flash temperatures. However, the relation may be less clear. Heat transfer between a product surface and the skin depends on the magnitude of the real contact area, hence on the topography of the surface. The same dependencies are found for the coefficient of friction.

Clear correlations are reported in the literature between roughness amplitude of the surface and the coefficient of friction. When touching a rough surface, the roughness produces an uneven pressure distribution on the skin and vibrations when the fingerpad slides over the surface. The perceived roughness of sandpapers is found to increase with particle diameter following a power law. The results reported by several authors show that for textured surfaces the perceived roughness increases when the distance between elements increases, i.e. when the asperity density decreases. For random rough surfaces, although correlations were found between average roughness amplitude $R_{a}$ and perceived roughness, it was found that peak values of the roughness profile are better descriptors to relate roughness to associated feelings. It may be concluded that there is not one single roughness parameter that correlates best to perceived roughness, but that roughness is a multidimensional sensation that is determined by roughness amplitude, asperity density and friction between the surface and the fingerpad. Several studies report a positive correlation between perceived roughness and friction.

The perception of slipperiness, or stickiness, relates to the frictional properties of a surface. Only a few studies have been carried out on the perception of slipperiness, finding a correlation with the measured coefficient of friction. Contact mechanics theory shows that the maximum tensile stress at the skin surface is proportional to the coefficient of friction. Furthermore, the magnitude of the maximum subsurface shear stress is proportional to the coefficient of friction, whereas its location, the distance underneath the surface, changes with friction. Various mechanoreceptors in the skin detect the variation and magnitude of these stresses when the fingerpad slides over a surface, giving rise to a certain perception of slipperiness. Figure 1.1 shows the relation between the interaction with the skin and the sensorial responses schematically. 

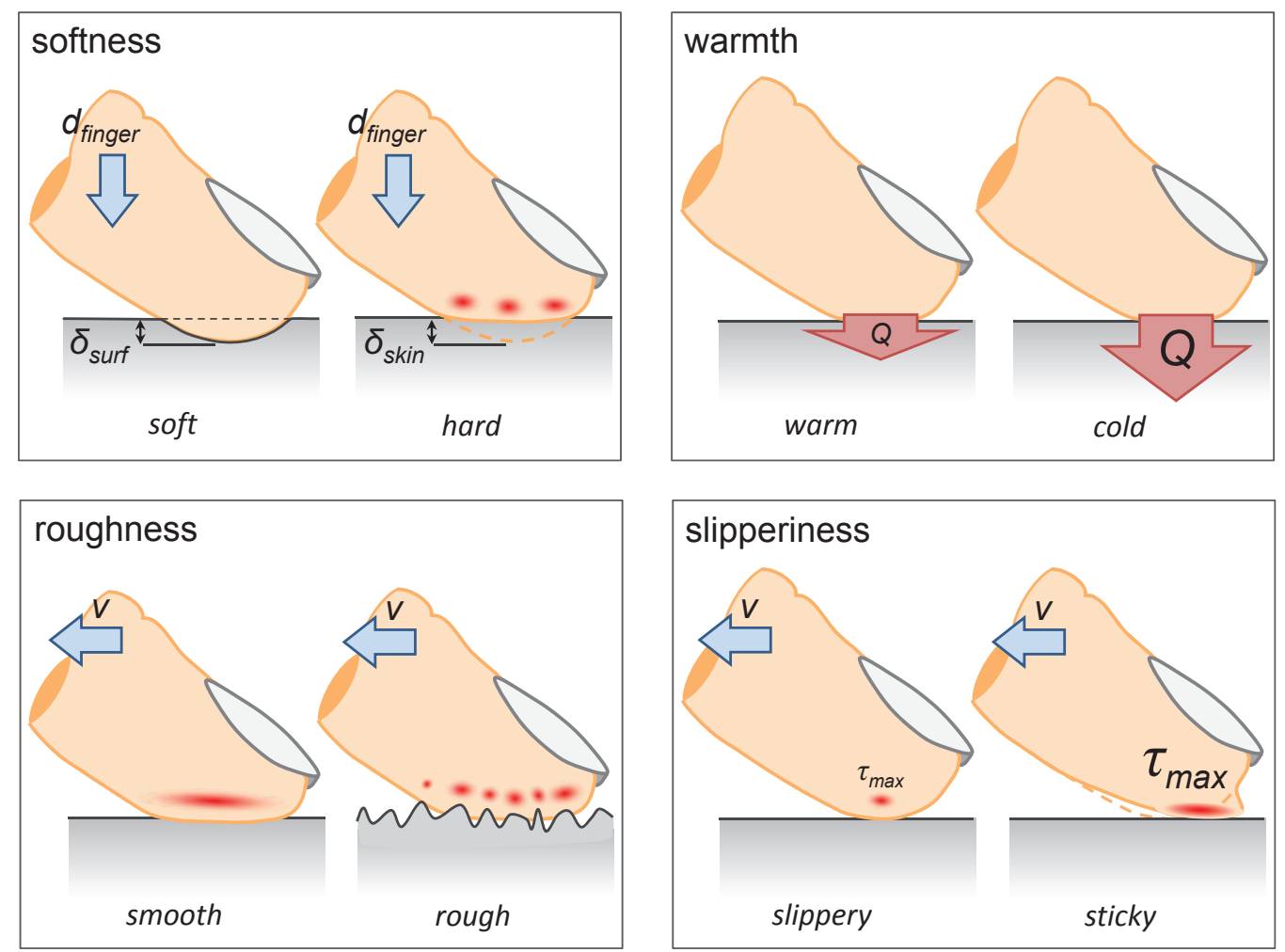

Figure 1.1: Schematic overview of the relation between the stimulus at the skin surface and the sensorial properties softness, warmth, roughness and slipperiness. 
In recent years,engineering tribology has shown interest in touch perception. One of the first studies on touch properties of surfaces published in tribology literature was carried out at the University of Leeds by Barnes et al. [2]. The main conclusion from their investigation of the sliding contact of a fingertip over glass surfaces with different roughnesses was that desirable feelings are generated when the finger slides over a surface which is less rough than the fingertip, whereas negative feelings are generated when the finger slides over a surface which is rougher than the fingertip. Following this publication a number of studies have appeared in tribology journals. The relation between surface - and material - properties of consumer products and consumer's judgments was the subject of further research by the Leeds group [3, 4]. Darden and Schwartz investigated the relation between the tribological behaviour and the tactile attributes of polymer fabrics [5]. Recently, several studies were carried out on the relation between friction properties and perceived surface properties, such as coarseness [6] and grip [7]. Understanding the relationship between the perceptual responses and properties of the material is the subject of affective engineering. To this end, the relation between the surface properties of the material, the friction behaviour against the skin, and the sensorial and affective responses is of interest.

\section{$1.2 \quad$ Skin tribology}

The science of tribology studies the contact between interacting surfaces in relative motion. One of the contact phenomena that occur between two surfaces sliding against each other is friction, a tangential force resisting motion. It is common to define 'friction' and 'the frictional behaviour' by the coefficient of friction which is calculated as the quotient of the friction force and the normal load. This coefficient of friction depends on the material combination, the micro-geometry of the surfaces, any lubricants and the environmental conditions, as well as the operational conditions of the contact, which together form the tribological system [8]. Figure 1.2 shows a schematic illustration of the tribological system of skin friction.

In skin tribology at least one of the interacting surfaces is the human skin, a living material. As a material, skin behaves in a complex manner; it 


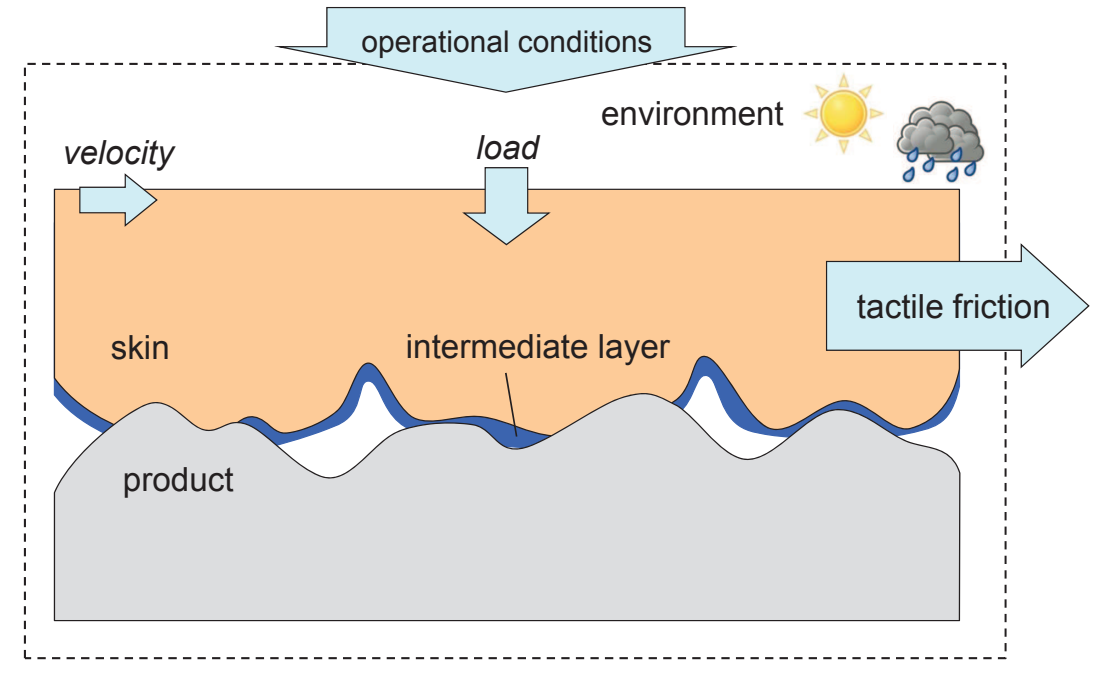

Figure 1.2: Schematic illustration of the tribological system of skin friction.

has a layered structure with highly changing properties through the layers, its behaviour is viscoelastic, anisotropic and there may be an influence of underlying tissue and bones. The properties of the skin vary with anatomical location and with subject, age, gender, level of care and hydration. A schematic illustration of the anatomy of the human skin is shown in Fig. 1.3. The outermost layer of the skin is the stratum corneum, which is actually the top layer of the epidermis, the first layer of the skin. The second layer is the dermis, which is supported by the much softer hypodermis, or subcutis. Underneath the hypodermis is the subcutaneous tissue; muscle, tendons and bone. The contact surface of the skin is formed by the stratum corneum, which is composed of cornified cells [9]. This layer has a high stiffness, which lies in the order of 10 to $1000 \mathrm{MPa}$ depending on hydration level [10, 11] and at the fingerpad has a thickness of several hundreds of micrometers as opposed to $10-20 \mu \mathrm{m}$ on the hairy skin. The surface topography of the fingerpad skin is formed by the so-called fingerprint ridges, a pattern of ridges and furrows having a width of approximately $200 \mu \mathrm{m}$ and $120 \mu \mathrm{m}$ respectively [12]. On the ridges the sweat glands are found with a surface density of $150-300$ per $\mathrm{cm}^{2}$ [13].

The friction force that occurs in the contact with the skin is a combination of the forces required to break the adhesive bonds between the two surfaces 
and the forces related to the deformation of the bodies in contact $[14,15]$. These forces occur in the contact spots which are formed between the asperity summits of the surfaces, which together constitute the so-called real contact area. The real contact area is only a fraction of the apparent contact area which is calculated using contact mechanics equations, such as provided by Hertz [16].

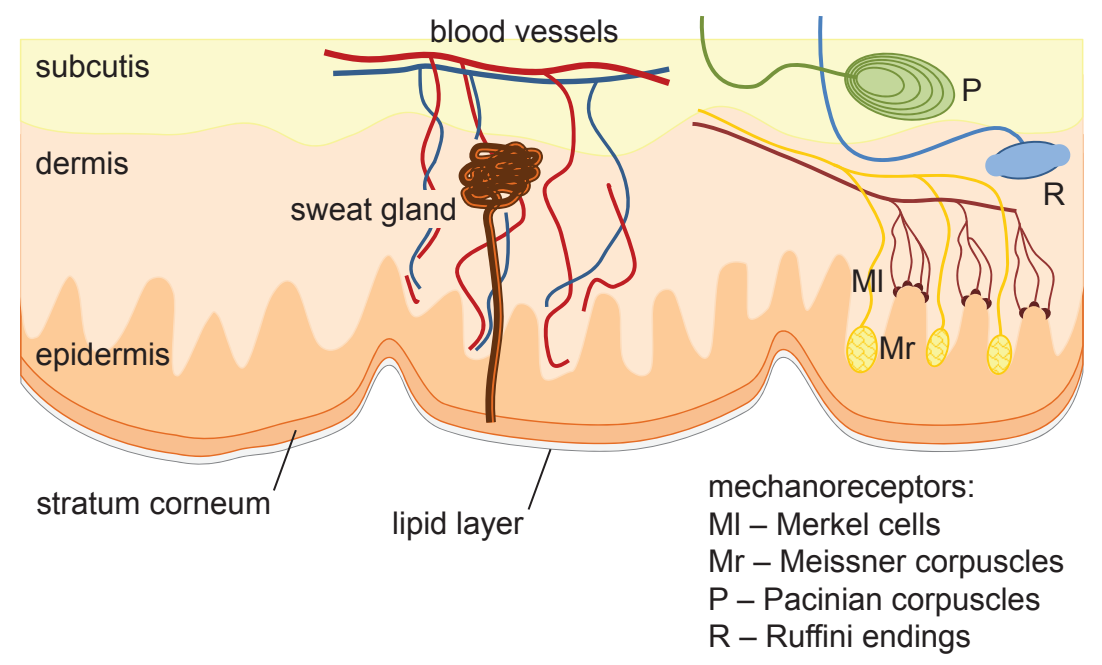

Figure 1.3: Anatomy of the skin of the human fingerpad.

\subsection{Tactile friction}

Friction in contacts where the human fingerpad is one of the interacting surfaces is referred to in this thesis as 'tactile friction'. Typically the applied pressing loads are low, in the order of several Newtons, as opposed to 'grip', where loads up to $50 \mathrm{~N}$ may be applied. In the sliding contact between the fingerpad and a rough surface when touching a product's surface, friction plays a role in the perception of roughness, slipperiness and warmth. For product engineers who aim to control and optimize the sensorial properties of a product surface interacting with the skin it is essential to understand this frictional behaviour. However, the frictional behaviour of skin is yet poorly understood. Numerous studies have reported on the friction behaviour of the human fingerpad. A wide range of coefficients of friction has been presented 
as a function of countersurface material, surface roughness, applied normal load and skin condition as shown in Paper A. Analysis of the data collected from the literature shows some consistent trends:

- The coefficient of friction increases considerably with increasing hydration level of the skin, due to softening of the top layer of the skin.

- The coefficient of friction against the fingerpad decreases with normal load to a constant value, which can be attributed to effects of normal adhesion and the deformation behaviour of the fingerpad.

- Friction decreases with increasing $R_{a}$-roughness. When the $R_{a}$-roughness increases further, the contribution of deformation causes an increase in the friction, after which it remains constant.

However, the collected coefficients of friction are the average coefficients of friction of the steady state part of the measuring signal, which have been obtained by dividing the measured friction force by the applied normal load. Consequently, any local effects, whether in the spatial or in the temporal domain, remain unobserved.

\subsection{Objectives of this research}

Understanding 'product feel' and the interaction of human skin with product surfaces requires a thorough knowledge of the tribological phenomena occurring at the interface between the human skin and the product surface. This involves the study of local friction behaviour at the scale of the surface roughness. This research aims at building an understanding of the friction behaviour of the human skin as a function of surface topography. To be able to control the frictional behaviour as a function of asperity geometry, a predictive model is required, which takes into account the effects of the layered composition, the surface topography and the condition of the skin. Furthermore, this research requires the development of an experimental method for measuring the friction of in vivo skin at the scale of the surface roughness. 
The objective of the research in this thesis is the development of a model for the contact and tactile friction behaviour of human skin as a function of countersurface asperity geometry and operational conditions.

\subsection{This thesis}

This thesis describes the development of a model for the contact and tactile friction behaviour of human skin as a function of countersurface asperity geometry and operational conditions. A mechanistic approach has been adopted in which analytical models from contact mechanics theory were used to develop a model which describes the friction behaviour against the human fingerpad. Sliding friction was measured in vivo between the skin of the human fingerpad and different surface textures produced using ultra-short pulsed laser technology. Figure 1.4 shows a schematic illustration of the selected approach. The sliding contact between the skin of the human fingerpad and rigid surface textures consisting of evenly distributed spherically-tipped asperities was investigated within the scope of tactile exploration.
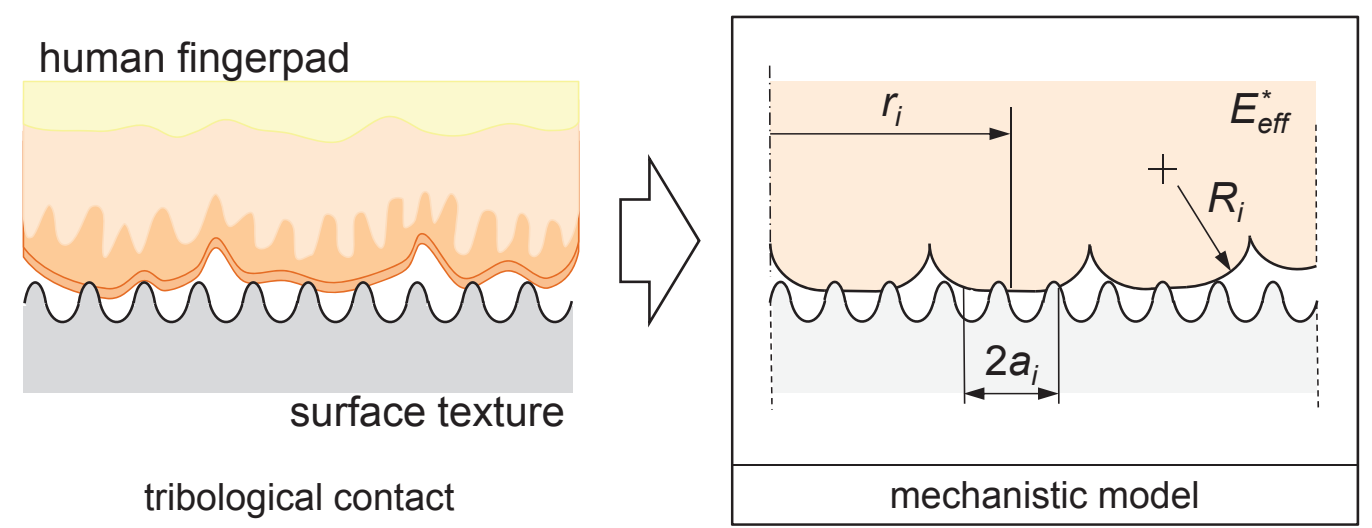

Figure 1.4: Schematic illustration of the tribological contact and the mechanistic approach used in this thesis.

This thesis is divided into two parts: Part I is an overview of theory presented in the papers appended in Part II. 
The chapter layout of Part I follows the mechanistic approach that was used in this research. The first step was the development of a contact and friction model for human skin, for which 'a rigid smooth sphere sliding against human skin' was choosen as a model system. Chapter 2 describes the development of the model and a comparison of the results to data collected from the literature. In Chapter 3, the multiscale model developed for the contact of a rigid smooth sphere is extended to a model describing the contact and friction behaviour of the human fingerpad sliding against a textured surface. For a rigid textured surface, the friction behaviour against the human fingerpad can be calculated as a function of asperity tip radius and asperity density. To investigate the friction behaviour as a function of asperity geometry experimentally, surface textures consisting of evenly distributed spherically-tipped asperities were produced. Chapter 4 describes the experiments as well as an investigation of the sources of variation which are intrinsic to in vivo experiments. Finally, the conclusions of this research and some recommendations for future research are presented in Chapter 5. 


\section{Chapter 2}

\section{Contact and Friction of Skin}

\subsection{Two-term friction model}

A set-up commonly used to investigate skin friction is a smooth spherical probe sliding against the skin [see e.g. 15, 17-25]. Figure 2.1 shows friction data for smooth spherical probes sliding against the skin collected from the literature. The measured friction force is plotted against the normal load applied on the probe.

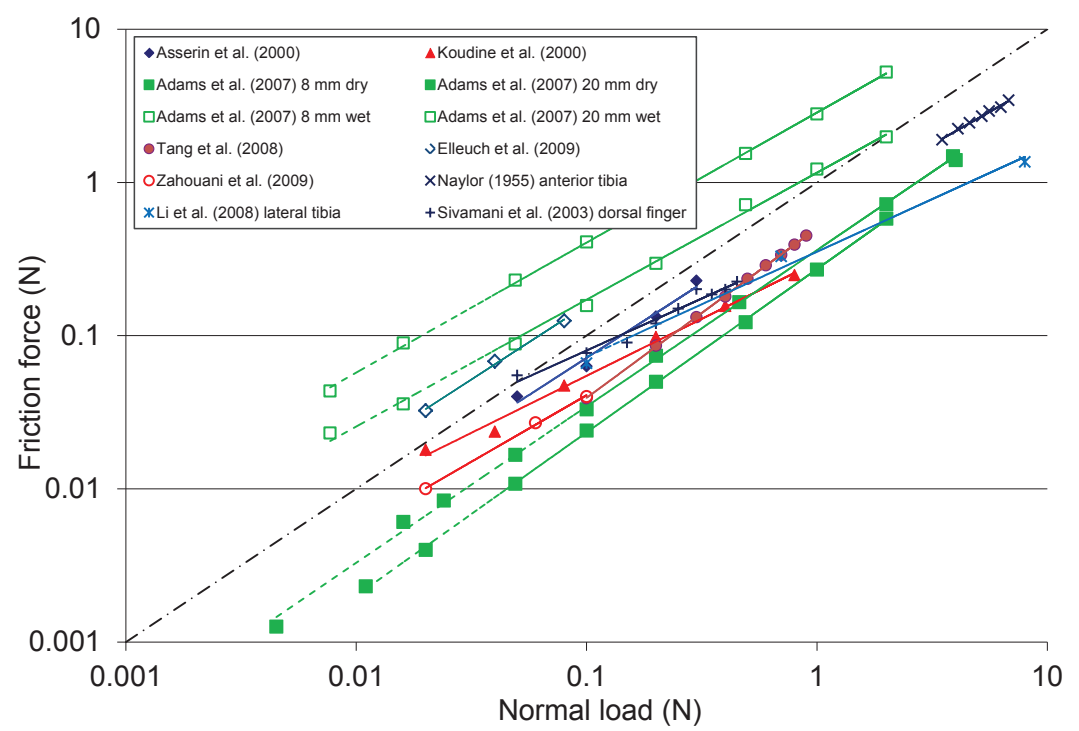

Figure 2.1: Measured friction force of a smooth spherical probe sliding against the skin of the human volar forearm unless stated otherwise. 
The coefficient of friction is defined as the quotient of the measured friction force and the applied normal load following

$$
\mu=\frac{F_{\mu}}{F}
$$

This simplified representation hides the fact that the friction that occurs in the contact is the result of different mechanisms. To a first approximation, Johnson et al. [14] and Adams et al. [15] give the total friction force of a spherical indenter sliding against the human skin by the sum of two non-interacting terms. This so-called two-term model gives the friction force as the sum of an adhesion term and a deformation term.

$$
F_{\mu}=F_{\mu, a d h}+F_{\mu, d e f}
$$

The first term in equation (2.2) is the force required to break the adhesive bonds between the two surfaces, which can be calculated from

$$
F_{\mu, a d h}=\tau A_{\text {real }}
$$

in which $\tau$ is the shear strength of the interface and $A_{\text {real }}$ is real contact area. When the surface asperities indenting the softer skin are moved forward, work is dissipated due to the viscoelastic nature of the skin. The deformation component equals the amount of work lost by viscoelastic hysteresis per unit sliding distance, thus being proportional to a viscoelastic loss fraction $\beta$ and the relative indentation of the asperities $\delta / a=a / R$ into the skin

$$
F_{\mu, d e f}=\frac{3}{16} \beta \frac{a}{R} F
$$

In many cases, the indentation behaviour of the skin can be assumed to be elastic, so that the contact theory developed by Hertz applies [11, 22, 26]. Hertz's theory [16, 27] is based on the assumption that the material is homogeneous and isotropic, and is valid for relatively small deformations. The dimensions of the Hertzian contact are determined by the geometrical and loading parameters and the elastic behaviour of the skin. By regarding the skin as an elastic half-space loaded over a small circular region of its plane surface, the contact radius $a$ and indentation depth $\delta$ can be calculated from 
the normal load $F$ and the radius of curvature of the body $R$ using Hertz's theory as shown in Equations (2.5) and (2.6) respectively

$$
\begin{gathered}
a_{\mathrm{H}}=\sqrt[3]{\frac{3}{4} \frac{R F}{E^{*}}} \\
\delta_{\mathrm{H}}=\sqrt[3]{\frac{9}{16} \frac{F^{2}}{R E^{* 2}}}
\end{gathered}
$$

in which $E^{*}$ represents the reduced elastic modulus, defined as

$$
\frac{1}{E^{*}}=\frac{1-\nu_{1}^{2}}{E_{1}}+\frac{1-\nu_{2}^{2}}{E_{2}}
$$

with $E_{1}$ and $E_{2}$ the respective elastic moduli and $\nu_{1}$ and $\nu_{2}$ the Poisson's ratios of the contacting materials. In the case of contact between skin and a stiff counterbody, $E_{\text {counterbody }} \gg E_{\text {skin }}$ and the reduced elastic modulus depends solely on the properties of the skin so that $E^{*} \approx E_{\text {skin }} /\left(1-\nu_{\text {skin }}^{2}\right)$.

Although theoretically described as the sum of two components, in skin tribology literature it is common to describe the relation between the measured friction force and the applied normal load as a power law so that

$$
F_{\mu}=F_{\mu, a d h}+F_{\mu, d e f} \approx k F^{n}
$$

When $n=1$, as indicated by the dash-dotted line Fig. 2.1, the coefficient $k$ equals the coefficient of friction $\mu$ as defined by Amontons' law of friction. Figure 2.1 shows that the measured friction force is approximately proportional to the applied normal load. The exponent $n$ in Equation (2.8) varies between 0.67 and 1.11. This behaviour can be explained using Hertz's theory [16], assuming the skin that is smooth. However, although the effect of the probe roughness of a smooth probe can be neglected, the contribution of the surface features of the skin to the contact behaviour should be considered. 


\subsection{Contact between a sphere and the skin}

When developing a contact model that predicts the contact area and indentation depth of the probe in contact with the skin, several difficulties are encountered. An essential input parameter in such a model is the elastic modulus, which is highly scale-dependent due to the layered composition of the skin. If possible at all, an exact description of the friction behaviour of the skin would thus require an anisotropic, nonlinear, viscoelastic model [28]. Furthermore, the distinctive skin microrelief hinders the use of statistical models to calculate the real area of contact. In this thesis, analytical models from contact mechanics theory are used to explain the observed friction behaviour of the smooth spherical probe sliding against the skin of the volar forearm.

\subsubsection{Length scale effect}

Data collected from the literature shows that the measured elastic modulus of the skin varies orders of magnitude with varying contact length scale. An overview is given in Paper B. At the length scale of surface roughness, i.e. smaller than $10 \mu \mathrm{m}$, the elastic properties of the skin are determined by the top layer of the skin. The elastic modulus of isolated stratum corneum decreases with indentation depth, reported values ranging for dry stratum corneum from $1 \mathrm{GPa}$ at the outer surface down to $3.44 \mathrm{MPa}[10,11,29]$. For wet stratum corneum, a considerably lower elasticity was measured decreasing from about $50 \mathrm{MPa}$ to $10 \mathrm{MPa}$ at $0.2 \mu \mathrm{m}$ and $2 \mu \mathrm{m}$ indentation, respectively [10]. Mechanical properties of the stratum corneum are strongly dependent on environmental temperature and relative humidity $(\mathrm{RH})$. It can be seen that the elasticity of the stratum corneum tends to decrease with increasing relative humidity [30-32]. At larger length scales, i.e. millimeters, the properties of the dermis and hypodermis determine the elastic behaviour of the whole skin. From indentation experiments carried out at the inner forearm using 4 to $8 \mathrm{~mm}$ radius indenters, elastic moduli ranging from about 5 to $53 \mathrm{kPa}$ were found for normal loads between 0.06 and $1 \mathrm{~N}$ and indentation depths between 0.6 and $3 \mathrm{~mm}$ [11, 14, 22, 26, 33-35].

To account for the multilayered and nonhomogeneous structure of the skin, as well as the relatively large deformations and any nonlinear effects 
in a relatively straightforward manner, it is suggested to use the effective elastic modulus $E_{\text {eff }}$, sometimes referred to as the apparent or equivalent elastic modulus. Although it might formally not be correct from a physical point of view, the concept of an effective elastic modulus is very useful in contact and friction modelling as it reduces a multiparameter expression to a single parameter. The effective elastic modulus is a combination of the respective moduli of the skin layers and of the underlying tissue such as muscles and bones. The relative contribution of each individual skin layer to the effective elastic modulus is determined by the ratio of the thickness of the layer $t$ to the length scale of the contact $a$.

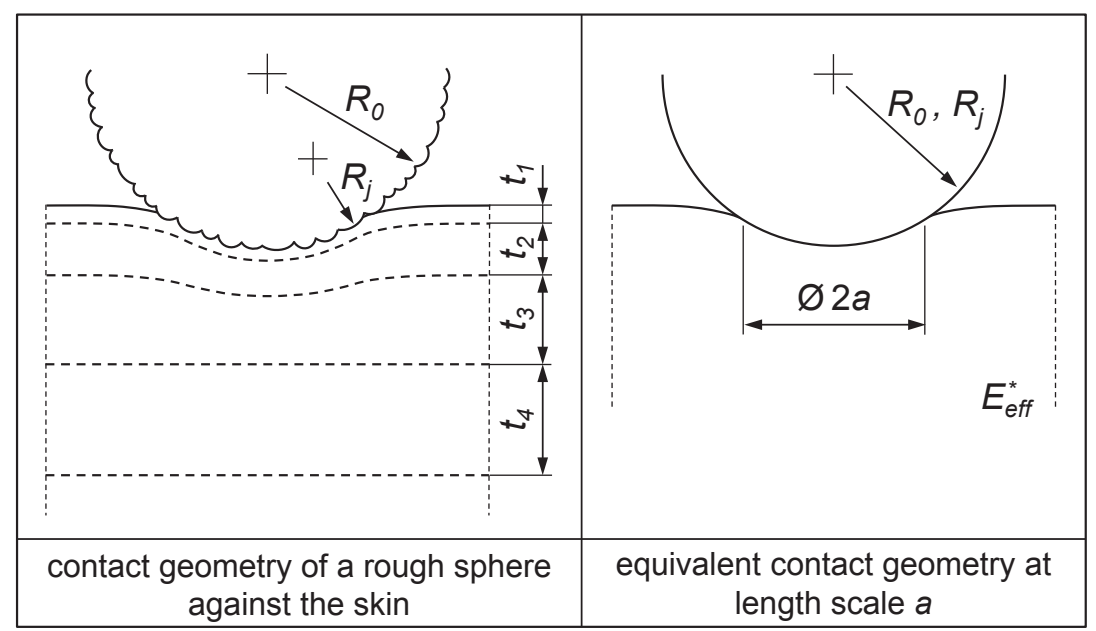

Figure 2.2: Schematic figure of the concept of the effective elastic modulus.

In the current study, the analytical single-layer model developed by Bec et al. [36] was extended to a four-layer model. The global stiffness of the skin $K_{g}$ is given as the reciprocal sum of the stiffnesses of the different skin layers $K_{i}$ and of the underlying tissue $K_{n}$. From the definition of the stiffnesses $K_{i}=\pi a^{2} E_{i}^{*} / t$ and $K_{n}=2 E_{n}^{*} a$, the effective elastic modulus is given as

$$
\frac{1}{E_{e f f}^{*}}=2 \sum_{i=1}^{n-1} \frac{t_{i}}{f_{i}(a) \pi a E_{i}^{*}}+\frac{1}{f_{n}(a) E_{n}^{*}}
$$

To ensure correct boundary conditions, the polynomial functions $f_{i}(a)$ were introduced in the expressions of the different moduli [36, 37]. 
- When the contact size is very small as compared to the thickness of the upper layer, the effective elastic modulus equals the elastic modulus of the upper layer.

- If the properties of the different layers are the same as those of the substrate, the effective elastic modulus equals the elastic modulus of the substrate.

- If the properties of neighbouring layers are the same, those layers respond as one thick layer with the same elastic properties.

In analogy to the work of Bec et al. [36] and Pailler-Mattei et al. [37], for the four-layer model this leads to the following expressions for the functions $f_{i}(a)$ :

$$
\begin{aligned}
f_{1}(a) & =1+\frac{2 t_{1}}{\pi a} \\
f_{i}(a) & =\left(1+\frac{2}{\pi a} \sum_{j=1}^{i-1} t_{j}\right)\left(1+\frac{2}{\pi a} \sum_{j=1}^{i} t_{j}\right), \text { for } i=2 \ldots n-1 \\
f_{n}(a) & =\left(1+\frac{2}{\pi a} \sum_{j=1}^{n-1} t_{j}\right)
\end{aligned}
$$

From Equations (2.9) and (2.10) it can be seen that the relation between the effective elastic modulus of the skin $E_{\text {eff }}^{*}$ and the size of the contact $a$ depends solely on the respective thicknesses $t_{i}$ and elastic moduli $E_{i}^{*}$ of the different skin layers, indicated by the subscripts $i=1 \ldots 4$, and the underlying tissue indicated by the subscript $n=5$. Table 2.1 shows the parameter values for layer thickness $t_{i}$ and elastic modulus $E_{i}^{*}$ that have been used to calculate the effective elastic modulus of the skin of the volar forearm $E_{e f f}^{*}$ as a function of contact length scale $a$. Average values were calculated from the ranges found in the literature as shown in Paper B.

Figure 2.3 shows the effective elastic modulus as a function of length scale calculated using the data from Table 2.1. The complex behaviour of the skin of the volar forearm can be characterized by a single curve describing the elastic behaviour as a function of the length scale of the contact. Although the model omits the nonlinear characteristics of the skin and does not take into account the presence of hair follicles, the different 
Table 2.1: Parameters used for calculating the skin effective elastic modulus of skin of the volar forearm.

\begin{tabular}{lllll}
\hline$i$ & Skin layer, tissue & & $\begin{array}{l}\text { Elastic modulus } \\
E_{i}^{*}(\mathrm{MPa})\end{array}$ & $\begin{array}{l}\text { Thickness } \\
t_{i}(\mathrm{~mm})\end{array}$ \\
\hline 1 & Stratum corneum & dry & 500 & 0.025 \\
& & wet & 30 & \\
2 & Viable epidermis & 1.5 & 0.095 \\
3 & Dermis & 0.02 & 1.4 \\
4 & Hypodermis & $2 \times 10^{-3}$ & 0.8 \\
5 & Muscle & 0.25 & \\
\hline
\end{tabular}

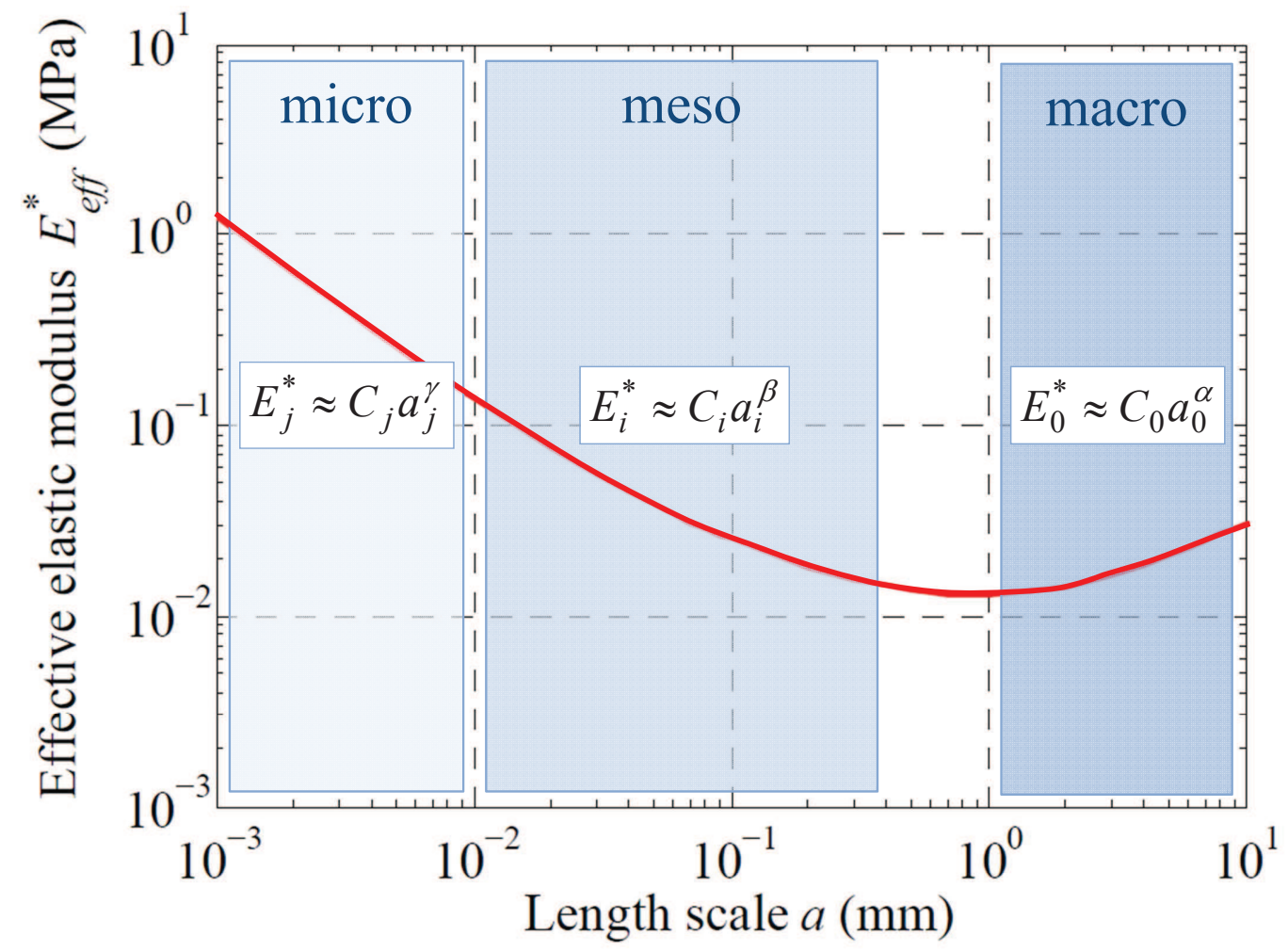

Figure 2.3: Effective elastic modulus of the skin of the volar forearm $E_{e f f}^{*}$ as a function of length scale $a$. At different scales the evolution of the effective elastic modulus with length scale can be described by power laws $(\alpha \approx 0.47$, $\beta \approx-0.64, \gamma \approx-0.96)$. 
glands and bloodvessels, the curve describes the evolution of the effective elastic modulus with the length scale of the contact rather well. The curve is compared to results collected from the literature in Paper B. The evolution of the effective elastic modulus with contact length scale depicted in Figure 2.3 is a consequence of the rather complex anatomy of the skin. At different length scales different layers contribute to the apparent elasticity. The elastic moduli of the respective layers decrease with depth. With increasing length scale, the contribution of the lower and softer skin layers to the effective elastic modulus increases, its magnitude being determined by the ratio of elastically deformed volumes $a^{3} / t_{i} a^{2}$, or relative length scale $a / t_{i}$. At small indentation depths, the effective elastic modulus decreases with increasing indenter radius or, in other words, increasing elastically deformed volume due to the increasing contribution of the lower and softer skin layers. After a decrease of several orders of magnitude with the length scale increasing from the micron to millimeter range, from a length scale of several millimeters a slight increase of the effective elastic modulus of the skin is caused by the underlying tissues such as muscle. Variations of properties of the lower and softer skin layers with age, gender or anatomical site give rise to variations in elastic properties. With increasing age, the reduced elastic modulus at the macroscale tends to decrease; for females the elasticity is lower than for males.

At different scales the evolution of the effective elastic modulus with contact length scale can be described using a power law, which enables substitution into the available contact mechanics formula. At the macro scale the elastic modulus of the skin varies with the contact radius following

$$
E_{0}^{*}=C_{0} a_{0}^{\alpha}
$$

Assuming that when normal loads are small the skin behaves elastically, the apparent contact area of the sphere in contact with the skin can be obtained from Equations (2.5) and (2.11) by

$$
A_{0}=\pi K_{0}^{2} F^{\frac{2}{3+\alpha}}
$$


where

$$
K_{0}=\left(\frac{3 R_{0}}{4 C_{0}}\right)^{\frac{1}{3+\alpha}}
$$

Equation (2.12) shows that when substituting the mechanical behaviour shown in Fig. 2.3, with $\alpha \approx 0.47$, the apparent contact area grows with the normal load following a power law with exponent 0.58 . It is widely assumed that adhesive friction is the dominant mechanism in the sliding contact between a rigid probe and the skin. The friction force due to adhesive shear is proportional to the real area of contact which is related to the skin microrelief.

\subsubsection{Skin microrelief}

The surface topography of the skin is characterized by primary and secondary furrows, which give shape to triangular and rectangular surface features of varying size. This distinctive surface topography is referred to as the skin microrelief, a pattern of plateaus and valleys. The depth of the primary lines varies between 20 and $100 \mu \mathrm{m}$, whereas the depth of the secondary lines varies between 5 and $40 \mu \mathrm{m}[38]$.

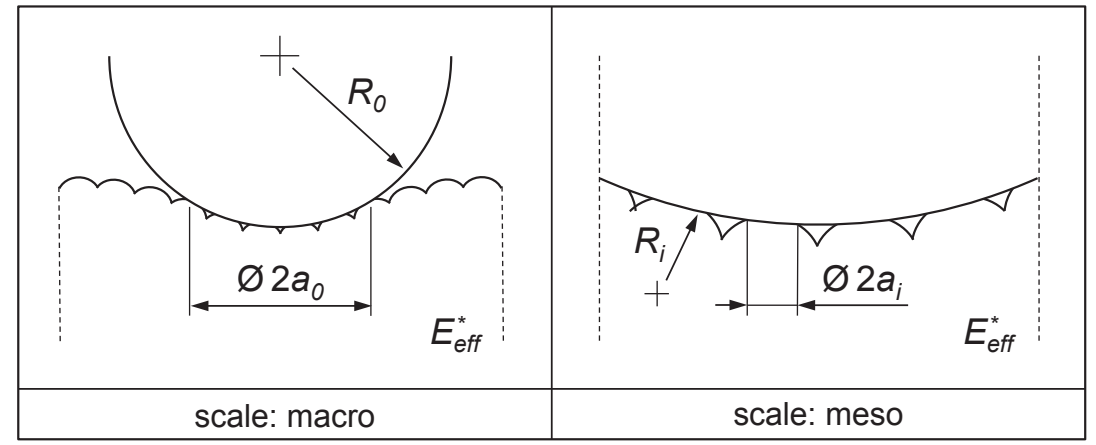

Figure 2.4: Contact dimensions of a smooth spherical probe in contact with the skin.

Although in most mechanical components the contact between two rough surfaces can be described by the contact between a flat and a surface having a so-called equivalent roughness, for skin contacts this assumption does not hold. In the contact between the skin and, in most cases, the 
stiffer product surface length scales and mechanical properties of the surface features are considerably different. This is why, the contact areas formed by the skin microrelief and the topography of the countersurface are calculated in subsequent steps. To calculate the real area of contact of an indenter sliding against the skin, the theory proposed by Archard [39] is followed, which takes into account the Hertzian pressure distribution in the macro-contact when calculating the normal loads at the asperity level. The skin microrelief is described as being composed of spherical contacts. The radius of curvature of the plateaus of the skin microrelief can be described as

$$
R_{i}=\frac{1}{\pi^{2} \eta R_{z}}
$$

where $\eta$ is the areal density of the surface features and the $R_{z}$-roughness is defined as the average distance between the highest peak and lowest valley in a given length. The areal density is the reciprocal of the average area of the plateaus of the surface features of the skin.

Areal density and average height of the surface features can be obtained from a 3D topography measurement of the skin. Typical values of the areal density $\eta$ found in the literature range between $\sim 9.1$ and $\sim 14.3$ per $\mathrm{mm}^{2}$ $[15,40,41]$ Boyer et al. [41] found that the areal density of the skin surface features decreases with increasing age. The $R_{z}$-roughness was reported to increase with age by Jacobi et al. [42], who reported values of 175, 194 and $228 \mu \mathrm{m}$ at average ages 25, 42 and 65 years respectively.

The Hertzian pressure distribution within the contact between the probe and the skin is given by $[16,27]$

$$
p(r)=\frac{3}{2} \frac{F}{A_{0}}\left(1-\frac{r^{2}}{a_{0}^{2}}\right)^{1 / 2}
$$

Equation (2.15) shows that the contact pressure decreases with radius from a maximum in the middle of the contact $(r=0)$ to zero at $r=a_{0}$ so that the normal load acting on an annulus of radius $r$ is given by

$$
\mathrm{d} F=2 \pi r p(r) \mathrm{d} r
$$

The number of skin surface features $N$ in contact with the spherical probe 
can be calculated from the nominal contact area $A_{0}$ and the density $\eta$ of the surface features of the skin microrelief. Following Archard [39], the normal load acting on each individual skin feature can be described as

$$
F_{i}=\frac{3}{2 \eta K_{0}^{2}} F^{\frac{1+\alpha}{3+\alpha}}\left(1-\frac{r^{2}}{a_{0}^{2}}\right)^{1 / 2}
$$

and the contact area between a single skin surface feature and the probe can be described analogous to Equation (2.12) as

$$
A_{i}=\pi K_{i}^{2} F_{i}^{\frac{2}{3+\beta}}
$$

where $K_{i}$ is a constant depending on the skin mechanical and geometrical properties defined analogous to Equation (2.13) and the effective elastic modulus at the meso scale is given by

$$
E_{i}^{*}=C_{i} a_{i}^{\beta}
$$

For a smooth spherical probe sliding against the skin, the real area of contact is determined by the behaviour of the skin microrelief. The real area of contact can now be calculated from

$$
A_{\text {real }}=\int_{r=0}^{r=a_{0}} A_{i} \eta 2 \pi r \mathrm{~d} r
$$

which leads to

$$
A_{\text {real }}=K_{1} F^{\frac{2}{3+\alpha}\left(\frac{1+\alpha}{3+\beta}+1\right)}
$$

with

$$
K_{1}=\pi^{2} \cdot \frac{3}{2 \pi}^{\frac{2}{3+\beta}} \cdot \frac{3+\beta}{4+\beta} \cdot K_{0}^{\frac{2(1+\beta)}{3+\beta}} \cdot \eta^{\frac{1+\beta}{3+\beta}} \cdot K_{i}^{2}
$$

Equation (2.21) shows that when substituting the mechanical behaviour shown in Fig. 2.3, with $\alpha \approx 0.47$ and $\beta \approx-0.64$, the real contact area grows with the normal load following a power law with exponent 0.94. Comparing this relation to the results summarized in Fig. 2.1 shows that its value lies close to the average trend seen in the literature.

The real contact area can now be calculated from Equation (2.21), which is based on Hertz's theory. However, since untreated human skin is covered 
with a lipid film, when studying the contact behaviour of the skin adhesive forces cannot be neglected [43].

\subsubsection{Adhesion}

The contact theory developed by Johnson, Kendall and Roberts [44] takes into account the surface forces due to adhesion. The JKR theory is applied to contacts of large, soft bodies with high surface energies. The adhesive force acting on a rigid asperity with tip radius $R$ equals the normal force necessary to separate the two surfaces and is given as

$$
F_{a d h}=\frac{3}{2} \pi R W_{12}
$$

The work of adhesion $W_{12}$ can be measured from indentation experiments or be approximated from the surface energy $\gamma_{i}$ of the materials [45] following

$$
W_{12} \approx 2 \sqrt{\gamma_{1} \gamma_{2}}
$$

For human skin, the work of adhesion was found to vary between 16 and $28 \mathrm{mN} / \mathrm{m}$ for radii of curvature 2.25 to $6.35 \mathrm{~mm}$ and normal loads between 20 and $80 \mathrm{mN}$ [43]. Water treatment increased the work of adhesion considerably $\left(W_{12}=107 \mathrm{mN} / \mathrm{m}\right)$ [43]. From Equation (2.23) it can be seen that the adhesive force acting on an asperity is independent of the elastic properties and the applied normal load. The apparent normal force acting on the asperity is given by [44] as

$$
F_{a p p}=F+2 F_{a d h}+2 \sqrt{F_{a d h}\left(F+F_{a d h}\right)}
$$

where $F$ is the externally applied normal load, so that for an asperity with radius $R$, the contact radius $a_{\mathrm{JKR}}$ is given by

$$
a_{\mathrm{JKR}}=\sqrt[3]{\frac{3}{4} \frac{R F_{a p p}}{E^{*}}}
$$

Due to the additional load on the contact the radius of the adhesive contact is larger than the radius of the non-adhesive contact predicted by Hertz. The

ratio of the contact radii depends on the ratio between the apparent normal 
load and the applied normal load following

$$
\frac{a_{\mathrm{JKR}}}{a_{\mathrm{H}}}=\left(\frac{F_{a p p}}{F}\right)^{1 / 3}
$$

\subsection{Friction between a sphere and the skin}

For a smooth probe sliding against the skin of the inner forearm, Equation (2.2) can be written as

$$
F_{\mu}=\tau A_{\text {real }}+\frac{3}{16} \beta \frac{a_{0}}{R_{0}} F
$$

so that after substitution of Equations (2.12), (2.21) and (2.27) the friction force can be calculated as a function of normal load.

Using the developed contact model, the friction force was calculated as a function of normal load for nine different cases collected from the literature, in which both measurement results and data on measurement conditions were provided. Table 2.2 summarizes the experimental data of the selected cases. Friction forces were calculated as a function of normal load, within the load ranges applied in the experiments. Based on the reported skin condition the value for the adhesion energy $W_{12}$ was assumed to be 0,20 and 100 $\mathrm{mN} / \mathrm{m}$ for 'cleaned', 'normal' and 'hydrated' skin respectively. To describe the mechanical behaviour of the skin, the generalized curve depicted in Fig. 2.3 was used. Input parameters in Equations (2.11) and (2.19) are $C_{0}=1 \cdot 10^{5}$, $\alpha=0.47, C_{i}=6$ and $\beta=-0.64$. For the viscoelastic loss fraction $\beta$ values varying between 0.24 and 0.43 are reported in the literature [14, 26, 41, 46]. The values for $\tau$ were chosen so that results for the coefficient $k$, which determines the magnitude of the average coefficient of friction, correspond to the values reported in the literature. This makes the interfacial shear strength $\tau$ the fitting parameter in this model. 
Table 2.2: Summary of experimental conditions for cases used for model calculations

\begin{tabular}{|c|c|c|c|c|c|c|c|c|c|}
\hline No. & Study & Material & $R_{0}(\mathrm{~mm})$ & $F(\mathrm{~N})$ & Skin & $T\left({ }^{\circ} \mathrm{C}\right)$ & RH (\%) & Age & Gender \\
\hline 1 & Asserin et al. [19] & ruby & 1.5 & $0.05-0.3$ & normal & $20-25$ & $40-50$ & 42 & female \\
\hline 2 & Koudine et al. [21] & glass & 10 & $0.02-0.8$ & normal & - & - & - & female \\
\hline 3 & Elleuch et al. [22] & steel & 6.35 & $0.02-0.08$ & normal & - & - & 25 & female \\
\hline $\begin{array}{l}4 \\
5\end{array}$ & Adams et al. [15] & PP & 20.2 & $0.005-5$ & $\begin{array}{l}\text { cleaned } \\
\text { wet }\end{array}$ & 20 & 50 & 30 & male \\
\hline $\begin{array}{l}6 \\
7\end{array}$ & & glass & 7.8 & $0.01-2$ & $\begin{array}{l}\text { cleaned } \\
\text { wet }\end{array}$ & 20 & 50 & 30 & male \\
\hline 8 & Tang et al. [24] & $\mathrm{PP}$ & 5 & $0.1-0.9$ & normal & $20-21$ & $50-60$ & $20-30$ & - \\
\hline 9 & Zahouani et al. [25] & steel & 6.36 & $0.02-0.1$ & normal & - & - & 40 & female \\
\hline
\end{tabular}

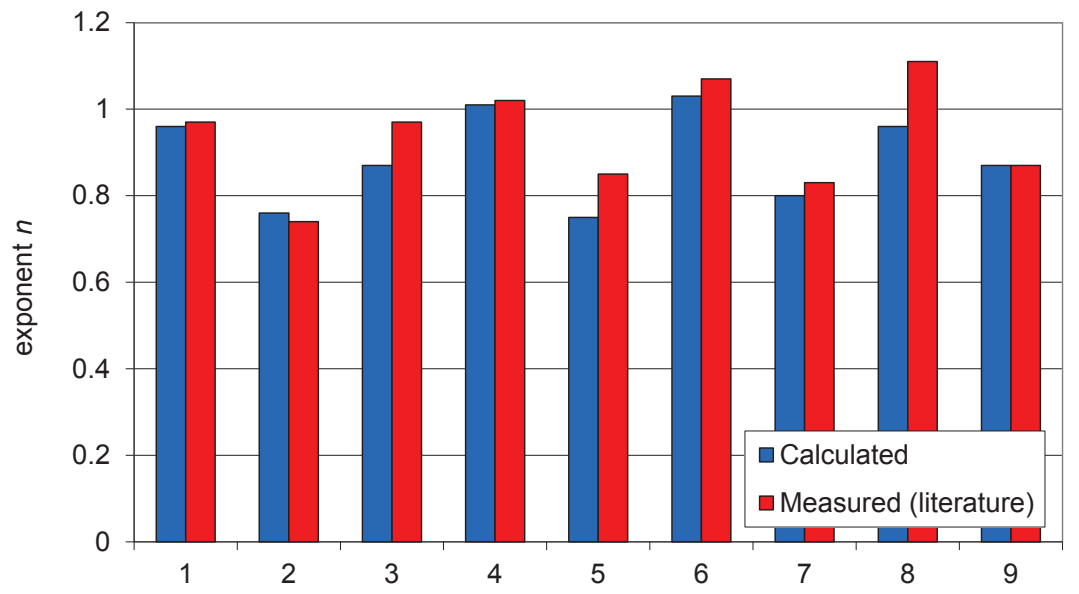

Figure 2.5: Comparison of model predictions with experimental results collected from the literature.

Figure 2.5 shows a comparison between measured and calculated values for the exponent $n$. The exponent $n$, which is commonly used to describe the relation between the friction force and normal load, i.e. the frictional behaviour, gives information about the mechanisms underlying the observed frictional behaviour. When the skin is cleaned, and free of surface lipids, the adhesion energy becomes negligible and Hertz's contact theory applies. Hence, the adhesion friction force is approximately proportional to the normal load. The contribution of a small, but increasing force due to deformation losses gives an exponent $n>1$. For untreated skin, the contribution of normal adhesion forces to the applied normal load is relatively large at low normal loads. With increasing normal load this contribution becomes less and, at high normal loads, negligible. Especially in the low 
normal load range, the increase of the measured friction force with applied normal load becomes less prominent, so that the exponent $n<1$. When the skin is hydrated, normal adhesion forces increase considerably and the exponent $n$ becomes even smaller, as can be seen in cases 5 and 7 , where $n \rightarrow 0.8$.

Calculations were carried out using a generalized set of input data, disregarding any differences between subjects in the different studies. One single relation was used to describe the mechanical behaviour of the skin, ignoring the effect of age and gender, which influences the evolution of the effective elastic modulus with length scale at the macro scale. Despite the approximate nature of the input parameters for the model calculations, the results of the calculations of the exponent $n$ correspond remarkably well to the values obtained from the reported measurements. The observed agreement between measured and calculated results for the relation between friction force and normal load shows that the two-term friction model is capable of describing the friction between a smooth spherical probe and the skin of the volar forearm. 


\section{Chapter 3}

\section{Modelling Tactile Friction}

\subsection{Review of fingerpad friction}

An analysis of experimental data collected from the literature, reported in Paper A, shows that the coefficient of friction measured against the human fingerpad parameter depends on changes in operational conditions, countersurface properties and measurement conditions. In engineering applications, the real contact area of the tribological contacts grows proportional to the normal load so that Amontons' law of friction applies, which states that the friction force is proportional to the normal load: the coefficient of friction has a constant value independent of normal load. Although several authors report a linear dependence of the friction force on normal load, others found that the coefficient of friction decreases with normal load following a power law with exponents between -0.68 and -0.24 . The behaviour might be different for different materials. Tomimoto [47] compared the influence of orientation of the finger on the friction behaviour. Whereas for the fingerpad the coefficient of friction decreases with increasing normal load, for the fingertip it remains approximately constant. The orientation of the finger is often referred to as 'pad', 'tip' or 'intermediate'. Furthermore, it was shown that the orientation angle has a significant influence on the magnitude of the coefficient of friction, the friction decreasing with increasing orientation angle. Warman and Ennos [48] showed that this can be attributed to the contact area and its relation to applied load. The evolution of the friction force with normal load can be explained partly by 
the growth of the apparent contact area. Several studies show that as a function of normal load the measured apparent contact area follows a power law with exponents ranging between 0.15 [49] and 1.33 [50]. Obviously, the relation between the contact area of the fingertip and normal load deviates from the behaviour predicted by Hertz [16], which gives an exponent 0.66 (see Paragraph 2.1).

Only a few studies report on the influence of sliding velocity on the measured friction. In general, the coefficient of friction of the fingerpad tends to decrease with increasing sliding velocity.

In most studies the surface topography of the sample is characterized by the $R_{a}$-roughness. For smooth surfaces, such as polished surfaces, the friction decreases with an increase of $R_{a}$-roughness. However, for rougher surfaces an increase is observed after which friction remains constant. Amplitude parameters such as the $R_{a}$-roughness are, in fact, statistical parameters describing the height distribution of the measured profile. Those parameters do not contain any information about size, shape or density of the asperities, properties that are the result of the finishing method. It is beyond doubt that these are the properties that define the surface topography and determine the frictional behaviour. Consistent results have been reported over the years on the effect of hydration. The coefficient of friction increases considerably with increasing hydration level of the skin. Up to threefold increases in coefficient of friction are reported in the literature. It should be noted that friction increases with an increasing hydration level of the skin, whereas above a certain level the presence of moisture at the skin surface reduces friction again.

\subsection{Contact between the fingerpad and a textured surface}

It was shown for a spherical probe in contact with the skin that the elastic behaviour can be described as a function of the contact length scale by a closed-form expression by using the concept of an effective elastic modulus. Due to the large variation in finger properties, consistent data sets of the mechanical properties of the fingerpad are not available. However, given the layered structure of the fingerpad skin, which is supported by the stiffer 
bone of the distal phalanx, the same trends in mechanical behaviour can be expected for the fingerpad.

When considering different size levels, for a spherical contact the evolution of the elastic modulus can be described as a function of the contact radius by a power law. At the macro scale, i.e. the fingerpad, the elastic modulus increases with increasing contact radius due to the increasing contribution to the effective elastic modulus of the much stiffer bone of the distal phalanx. At the scale of surface roughness, at contact radii of several micrometers, the elastic modulus is determined mainly by the stratum corneum, thus being almost independent of the length scale $a$. In between, the meso scale is the level of the fingerprint ridges. A multiscale model describing the friction between the fingerpad and a textured surface is reported in Papers C and D.

\subsubsection{Fingerprint area}

At the macro scale, the elastic modulus of the fingerpad varies with the contact radius following

$$
E_{0}^{*}=C_{0} a_{0}^{\alpha}
$$

Assuming that when normal loads are small the fingertip behaves as an elastic sphere, the nominal contact area of the fingerpad can be obtained from Equations (2.5) and (2.11) by

$$
A_{0}=\pi K_{0}^{2} F^{\frac{2}{3+\alpha}}
$$

where

$$
K_{0}=\left(\frac{3 R_{0}}{4 C_{0}}\right)^{\frac{1}{3+\alpha}}
$$

In Paper A an overview of data collected from the literature is given, showing that the apparent contact area grows with applied normal load following a power law, so that $A_{0} \propto F^{n}$ analogous to Equation (3.2). The exponent of the power law fitted through the measurement results depends on the range of applied normal loads. At higher loads, the apparent elastic modulus of the fingerpad increases with normal load and the exponent is smaller than the $2 / 3$ according to Hertz's theory. At very low loads, the relation between normal load and apparent contact area follows a power law with an exponent 
larger than 2/3. At normal loads around 1 Newton, the exponent $n$ varies between 0.27 and 0.58 . The growth of the contact area with normal load was further found to depend on the finger (index, ring etc.) and orientation of the finger; tip, pad or intermediate.

Figure 3.1a plots the apparent contact area $A_{0}$ as a function of normal load $F$. An exponent $n \approx 0.36$ was obtained experimentally by measuring the contact area of the fingerpad (using an inkpad) for various values of the applied normal load. Using Equation (3.2) this gives $\alpha=2.56$, showing that at the macro scale the effective elastic modulus increases strongly with the contact length scale. From Equations (3.1) and (3.3) the effective elastic modulus can be calculated, which at $1 \mathrm{~N}$ normal load, is approximately $47 \mathrm{kPa}$, which is comparable to values obtained by other researchers as shown in Paper A.
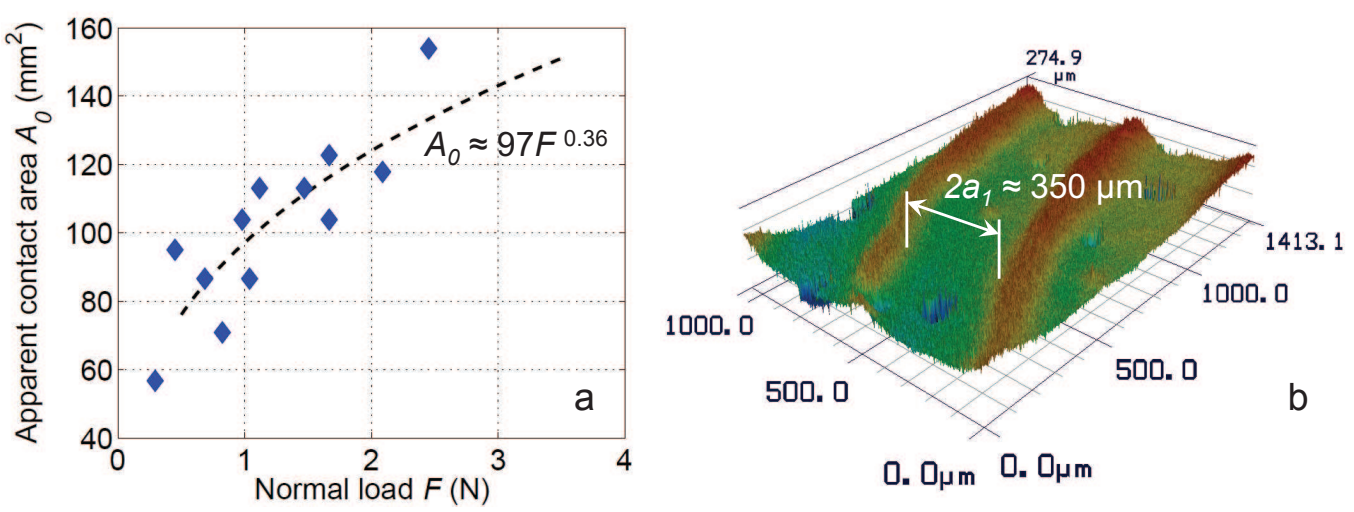

$0.0 \mu \mathrm{m} \quad 0.0 \mu \mathrm{m}$

Figure 3.1: Apparent contact area $A_{0}$ of the fingerpad measured as a function of normal load $F$ (a) and width and topography of a (replica of a) fingerprint ridge (b). Figure taken from Paper E.

In a next step, the fingerprint ridges are modelled as annulus-shaped line contacts. Surface topography measurements show that the shape of the fingerprint ridges is approximately trapezoidal. Although different ridge patterns (such as arch, loop, symmetrical and spiral) exist, to a first approximation the contacting areas between the fingerprint ridges and the countersurface are calculated by describing these contacts as annulus-shaped contacts. Assuming that the contact area is determined by the number of ridges that are in contact rather than deformation of the ridges [12], the 
contact area can be determined from the ridge density $\eta$ and ridge width $2 a_{1}$. From the number of fingerprint ridges in contact with the skin $\eta a_{0}$ the total length of the contacts is calculated as

$$
l=\pi \eta a_{0}^{2}
$$

which gives for the apparent contact area of the fingerprint ridges

$$
A_{1}=2 \pi \eta a_{1} K_{0}^{2} F^{\frac{2}{3+\alpha}}
$$

The topography of the skin of the fingerpad, the fingerprint ridges, was obtained from a replica made using a synthetic rubber replica compound (Compound 101RF, Microset Products Ltd, UK) as shown in Fig. 3.1b. The width of the fingerprint ridges varies between 300 and $400 \mu \mathrm{m}$, whereas the width of the furrows is $210-280 \mu \mathrm{m}$. The average density of the fingerprint ridges obtained from these values $\eta \approx 1.680 / \mathrm{mm}$. The amplitude of the surface features is $60-80 \mu \mathrm{m}$. These results give a contact ratio $A_{1} / A_{0}$ of approximately 0.59 , which corresponds to values reported in the literature which range from 0.27 to 0.59 as shown in Paper A.

When in contact with a surface texture which is composed of spherically-tipped asperities, as described in Paragraph 4.1.1, the real area of contact is determined by the contact behaviour at the micro scale.

\subsubsection{Regular surface textures}

When the asperities are arranged in a regular pattern having a spacing $\lambda$, the number of surface features $N$ in contact with the fingerpad can be calculated from the contact area $A_{1}$ following

$$
A_{1}=N \lambda^{2}
$$

Since the contact between the asperities and the fingerprint ridges is formed by the stratum corneum, the top layer of the skin, the size of the real contact area is determined by the properties of this top layer. The most important characteristic of this layer is the dependence of its properties on the hydration level. With the hydration level increasing, the elasticity of the stratum corneum reduces by several orders of magnitude, leading to an 
increase of the contacting area and hence of the measured friction. Although the glabrous skin of the hand does not contain any sebaceous glands, it does have a high density of sweat ducts supplying the skin of the fingerpad with a small amount of sweat to enhance grip by increasing adhesion. So, in contrast to the apparent fingerprint area, which is determined by the applied load or pressing force, the real contact area is determined by an apparent normal load $F_{a p p}$, given by Equation (2.25), which is a combination of the applied load and adhesive forces between the skin and the contacting surface. The contact ratio and relative indentation for the contact between the fingerpad and a regular surface texture can now be described as the following

$$
\frac{A_{\text {real }}}{A_{0}}=\pi\left(2 a_{1} \eta\right)^{\frac{1+\gamma}{3+\gamma}}\left(\frac{3}{4 C_{i}}\right)^{\frac{2}{3+\gamma}} R_{j}^{\frac{2}{3+\gamma}} \lambda^{\frac{-2(1+\gamma)}{3+\gamma}}\left(\frac{F_{a p p}}{A_{0}}\right)^{\frac{2}{3+\gamma}}
$$

and

$$
\frac{a_{j}}{R_{j}}=\left(2 a_{1} \eta\right)^{\frac{-1}{3+\gamma}}\left(\frac{3}{4 C_{i}}\right)^{\frac{1}{3+\gamma}} R_{j}^{\frac{-(2+\gamma)}{3+\gamma}} \lambda^{\frac{2}{3+\gamma}}\left(\frac{F_{a p p}}{A_{0}}\right)^{\frac{1}{3+\gamma}}
$$

Adhesive friction and deformation friction are proportional to, respectively, the real contact area and relative indentation.

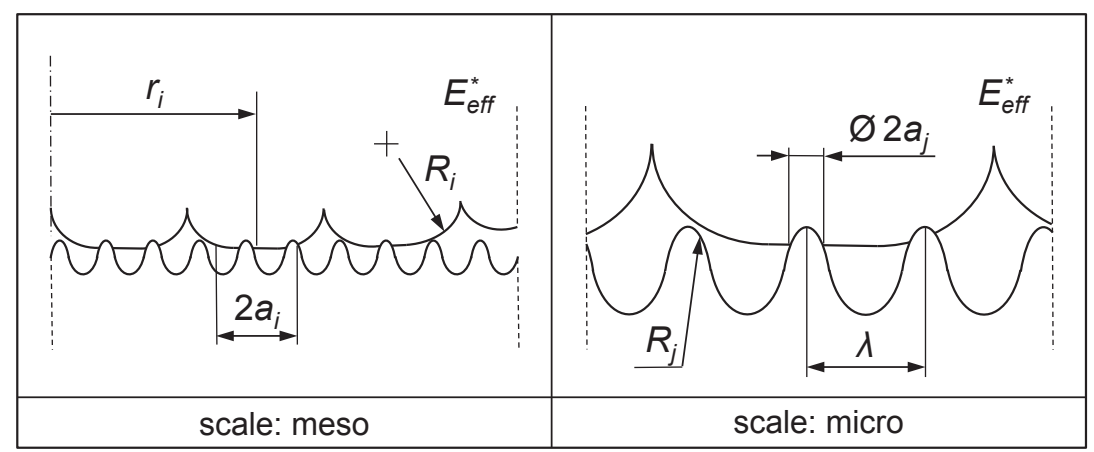

Figure 3.2: Contact dimensions of the fingertip ridges in contact with a regular surface texture. 


\subsection{Friction between the fingerpad and a textured surface}

For the fingerpad sliding against a regular surface texture, the friction force can be calculated as the following

$$
F_{\mu}=\tau A_{\text {real }}+\frac{3}{16} \beta \frac{a_{j}}{R_{j}} F
$$

so that after substitution of Equations (3.7), (3.8) and (2.27) the friction force as a function of texture characteristics and normal load can be calculated.

Figure 3.3 shows the calculated contact ratio and relative indentation as a function of texture characteristics. Considering the fact that adhesive shear is the dominant friction mechanism, the friction behaviour can be explained from the contact model, which shows that the real contact area increases with asperity tip radius and decreases with tip spacing. Furthermore, the contribution of normal adhesion to the effective normal load on the asperities increases with increasing tip radius, thus increasing the coefficient of friction. This effect becomes less pronounced for larger spacing. At a constant normal load the force acting on each asperity increases with increasing spacing so that the relative contribution of the normal adhesion to the effective normal load decreases. Since the indentation depth increases with spacing, after reaching a minimum the coefficient of friction will increase with spacing due to the growing contribution of deformation to the total friction force. 

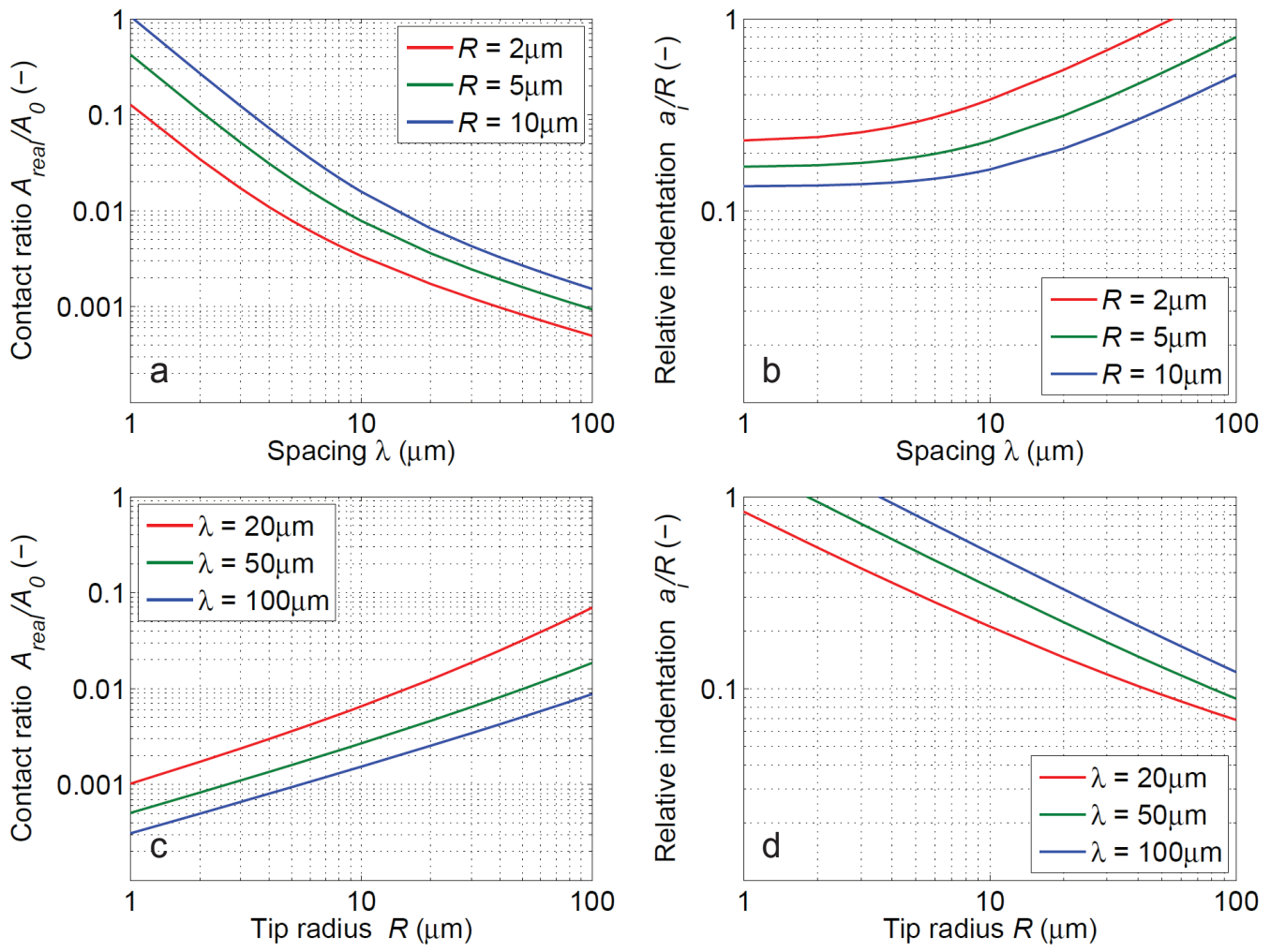

Figure 3.3: Contact ratio $A_{\text {real }} / A_{0}$ and relative indentation $a_{i} / R$ calculated as a function of spacing $\lambda$ for different tip radii $R(\mathrm{a}, \mathrm{b})$ and as a function of tip radius $R$ for different spacing $\lambda(\mathrm{c}, \mathrm{d})$ at $F=1 \mathrm{~N}$. 


\section{Chapter 4}

\section{Measuring Tactile Friction}

\subsection{Experimental methods}

A well-accepted method for measuring tactile friction is the principle of a stationary sample that is attached to a load measuring device, over which the subjects slide their fingerpad. The execution of these friction experiments is straightforward, so that several researchers used this principle in combination with questionnaire research to investigate the relation between friction properties and the various aspects of tactile perception. An overview of these studies is given in Paper A. When investigating friction of rough surfaces, a common approach is the focus on the friction behaviour of a single asperity as a first step, followed by the investigation of the multi-asperity contact.

\subsubsection{Surface textures}

The complicated scale dependence of roughness makes it difficult to pinpoint the friction or feel to a certain geometric parameter [2]. The amplitude parameters which are commonly used to characterize a surface's roughness, are in fact statistical parameters describing the height distribution of the measured surface profile. Those parameters do not contain any information about size, shape or density of the asperities. Because of the motion of the skin caused by, for example, blood circulation, carrying out experiments in vivo at the level of a single asperity, which is generally at the micrometer scale, is deemed impossible. By producing well defined surface topographies these obstacles can be avoided [3]. 


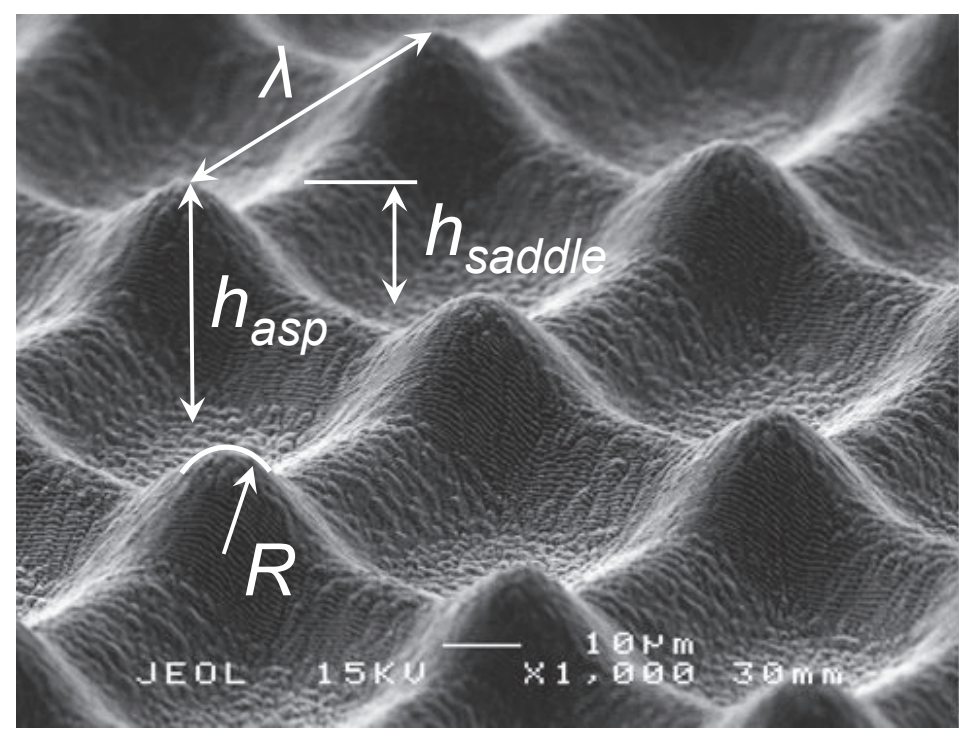

Figure 4.1: SEM image of a laser-textured sample with tip radius $R=5 \mu \mathrm{m}$ and spacing $\lambda=50 \mu \mathrm{m}$ (courtesy of Lightmotif, the Netherlands).

To investigate the friction behaviour as a function of asperity geometry, surface textures consisting of evenly distributed spherically-tipped asperities were produced using ultra-short pulsed laser technology (Lightmotif, the Netherlands). Figure 4.1 shows a SEM image of a representative sample. The surface textures are composed of bumps having a spherical tip with radius $R$, which are evenly distributed with a spacing $\lambda$ between the tips. A series of 6 samples was produced with tip radii of 2, 5, 20 and $20 \mu \mathrm{m}$ and spacings varying between 20 and $200 \mu \mathrm{m}$. The height of the asperities is $20 \pm 2 \mu \mathrm{m}$, the height of the saddle points is $10 \pm 2 \mu \mathrm{m}$. Surface topography was measured using a 3D Laser Confocal Microscope model VK-9700 (Keyence, Japan). Tip radii of the asperities were obtained from linescans of the 3D surface profile. The asperities have a conical shape with a spherical tip, as can be seen from Fig. 4.1. Table 4.1 summarizes the characteristics of the textured samples. The textures were applied onto flat stainless steel samples measuring 20 by $40 \mathrm{~mm}$ for in vivo friction experiments. More details can be found in Papers D and E. 
Table 4.1: Characteristics of the textured samples used in the in vivo experiments

\begin{tabular}{ccccc}
\hline$R(\mu \mathrm{m})$ & $\lambda(\mu \mathrm{m})$ & $R / \lambda(-)$ & $h_{\text {asp }}(\mu \mathrm{m})$ & $h_{\text {saddle }}(\mu \mathrm{m})$ \\
\hline 2 & 20 & 0.1 & 18 & 9 \\
5 & 20 & 0.25 & 18 & 9 \\
5 & 50 & 0.1 & 22 & 12 \\
5 & 100 & 0.05 & 24 & 12 \\
10 & 100 & 0.1 & 24 & 12 \\
20 & 200 & 0.1 & 25 & 11 \\
\hline
\end{tabular}

\subsubsection{Friction measurement}

The sliding friction between the fingerpad and the samples was measured using a load cell model ATI Gamma (ATI Industrial Automation, USA) as shown schematically in Fig. 4.2. The resolution of the 3-axis force/torque sensor is $25 \mathrm{mN}$ in the normal direction and $12.5 \mathrm{mN}$ in tangential direction, with a sample frequency of $100 \mathrm{~Hz}$. Samples were attached to the load cell using double-sided adhesive tape. Measurements were carried out by sliding the index finger over the sample in the horizontal plane. The normal load can be applied by pressing the finger onto the sample, while visually monitoring the measured normal load on the computer display. Improved load stability has been observed when a dead weight is attached to the finger. In this study, weights of $100 \mathrm{~g}$ and $200 \mathrm{~g}$ respectively were attached to the finger using double-sided adhesive tape, yielding normal loads of approximately $1 \mathrm{~N}$ and $2 \mathrm{~N}$. Normal loads exceeding this value were applied actively by the subject, these weights being too large to attach to the finger. The sliding velocity was approximately $10 \mathrm{~mm} / \mathrm{s}$. All tests were carried out at a room temperature of $20 \pm 2^{\circ} \mathrm{C}$ and relative humidity of $45 \pm 5 \%$. The coefficient of friction was calculated as the quotient of the measured friction force $F_{\mu}$ and normal load $F_{n}$ following equation (2.1). The normal load equals the load measured in vertical direction $F_{n}=F_{z}$, whereas the friction load is calculated from the loads measured in the lateral $x$ - and $y$-directions following

$$
F_{\mu}=\sqrt{F_{x}^{2}+F_{y}^{2}}
$$




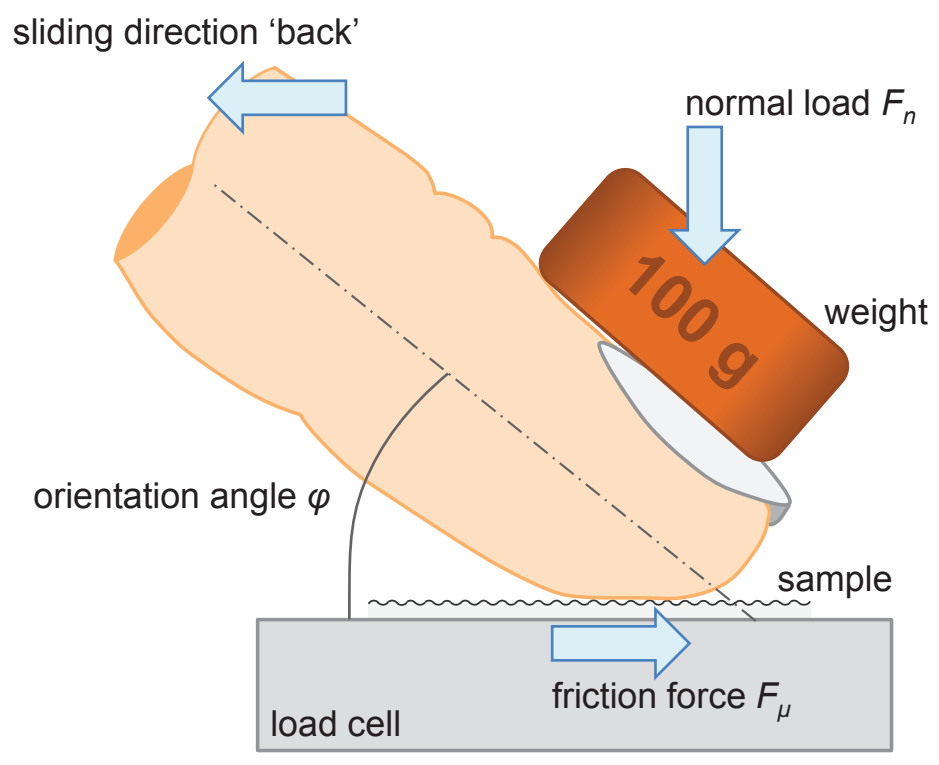

Figure 4.2: Experimental set-up for measuring the sliding friction between the fingerpad and textured samples.

Before the experiments the hands were washed using water and soap and air dried. After a rest period of approximately $10 \mathrm{~min}$. to allow the washed skin to regain its normal condition, the experiments were carried out. Experiments on hydrated skin were carried out after submersion in water for at least 10 min. Before the experiments any excess water was removed using a paper tissue. In its normal state, i.e. untreated, the skin may be referred to as dry. Damp skin is the skin after submersion and wiping off of free water. With damp skin, the top layer of the skin is hydrated, while there is no free water left on the surface of the skin.

Before and during the measurements the hydration level of the skin surface is monitored using a Corneometer CM 825 (Courage+Khazaka GmbH, Germany). The Corneometer measures the changing capacitance of a precision capacitor, which represents the change in the dielectric constant due to hydration of the skin surface, up to a measurement depth of 10-20 $\mu \mathrm{m}$. It reports the hydration of the skin on a scale of 0 to approximatley 100, in arbitrary units. The hydration of the skin has been linked to increasing friction by e.g. Cua et al. [51], Gerhardt et al. [52] and Kwiatkowska et al. [53]. 
The topography of the skin surface, the skin microrelief, was obtained from a replica made using a synthetic rubber replica compound (Compound 101RF, Microset Products Ltd, UK) on the area used in the tribological experiment. Surface roughness parameters were measured using a 3D Laser Confocal Microscope model VK-9700 (Keyence, Japan).

\subsubsection{In vivo experiments}

When carrying out experiments in vivo, the condition of the living skin will depend on the temperature and humidity of the environment, which are seldom controlled, the time of day and other personal aspects [54]. Obviously, this variation in conditions will lead to a variation in measured coefficients of friction. To be able to draw the correct conclusions on the relation between the surface parameters and frictional behaviour of the skin, one needs to understand the influence of conditions such as the measuring method and skin cleanliness and hydration level.

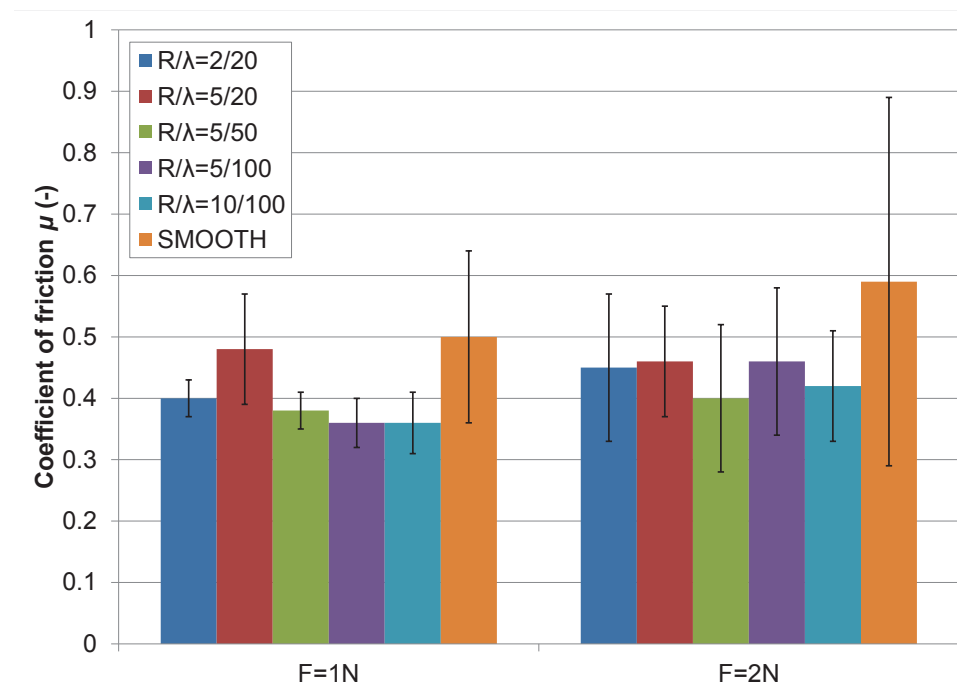

Figure 4.3: Coefficient of friction of the index finger as a function of normal loads for different texture parameters.

Since in general we explore surfaces with more fingers than just the index finger, the variation in frictional response has been evaluated. Figure 4.4 compares the frictional behaviour as a function of texture characteristics for 
the index finger, middle finger and ring finger. The skin of the fingers is in its normal state. The friction measured against the ring finger is considerably higher than the friction measured against the other fingers. The average hydration level of the ring finger during the tests was $66 \mathrm{AU}$, as opposed to 42 and $51 \mathrm{AU}$ for the index finger and middle finger respectively. Furthermore, the difference in geometry of the different fingertips causes a variation in the measured friction behaviour between the different fingers.

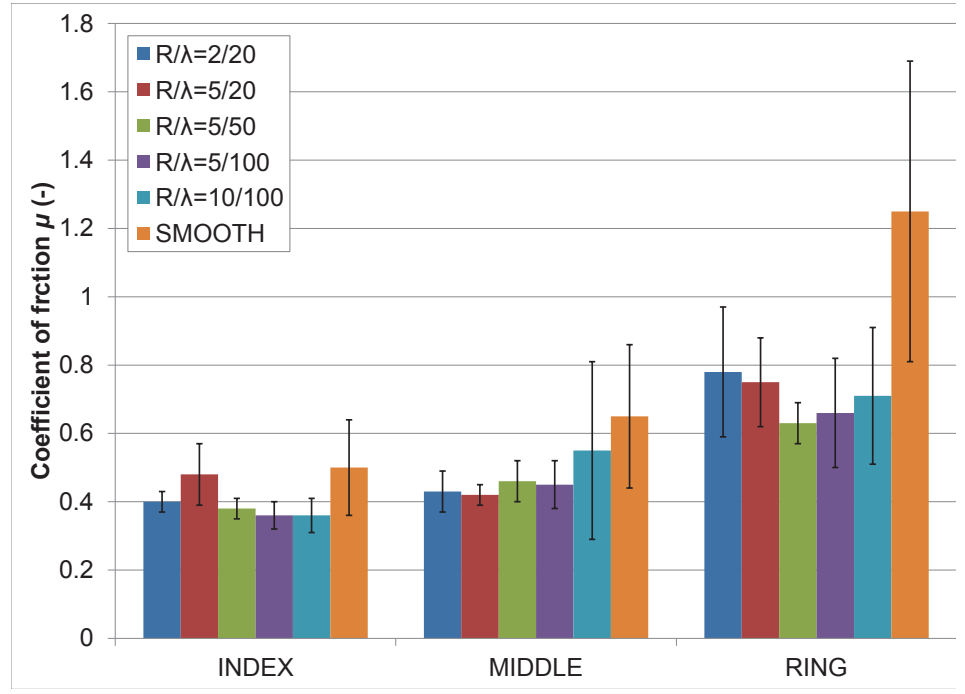

Figure 4.4: Coefficient of friction of untreated skin for different fingers at $1 \mathrm{~N}$ normal load for different texture parameters.

While sliding over a surface, the finger might have different orientations, varying from parallel to the surface i.e. using the fingerpad to perpendicular i.e. using the fingertip. Figure 4.5 shows the coefficient of friction as a function of texture characteristics and orientation of the fingerpad. The figure shows that the coefficient of friction increases significantly with decreasing orientation angle. The orientation angle being defined as 0 for the fingerpad and $\sim 80^{\circ}$ for the fingertip, as shown in Fig. 4.2. The decrease in measured friction with increasing orientation angle can be attributed to the reduction of the contact area with increasing angle [47, 48].

Figure 4.6 shows there is a slight dependence of the measured friction on the stroking direction, the coefficient of friction tending towards lower values in the forward direction. Although the average coefficient of friction was 


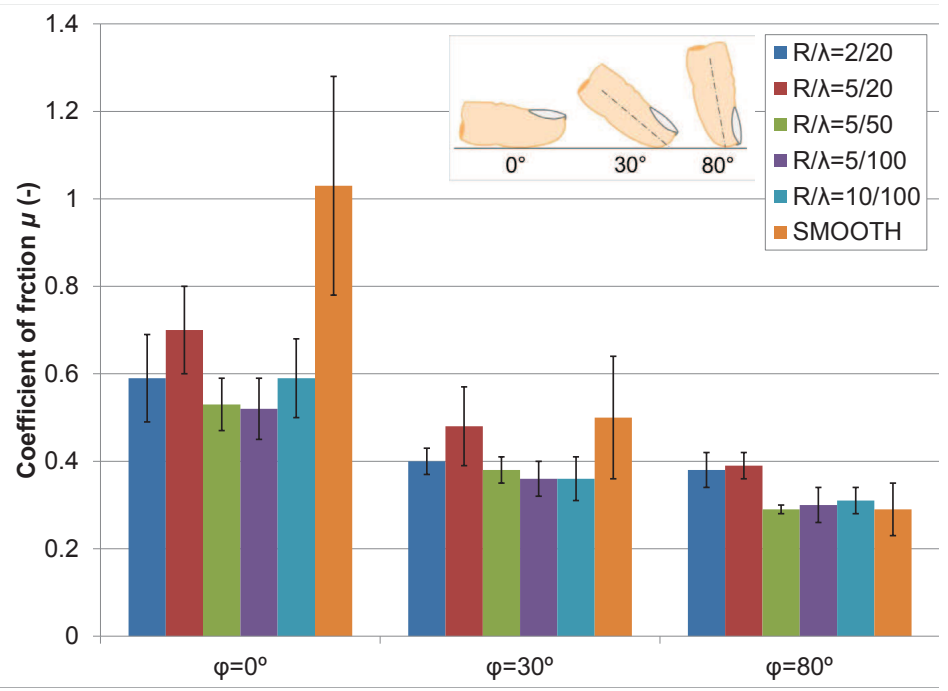

Figure 4.5: Coefficient of friction of the index finger for different orientation angles of the finger at $1 \mathrm{~N}$ normal load for different texture parameters.

observed to be lower, in the forward direction the sliding of the fingerpad was prone to stick-slip behaviour. The intermittent sticking, which was clearly noticed during the measurements, causes an irregular measurement signal characterized by large differences in friction between the sticking and the sliding phases.

During the experiments sweat, dead cells and other possible contamination are transferred from the skin of the fingerpad onto the countersurface. Figure 4.7 shows the coefficient of friction measured against the fingerpad as a function of the number of repeated sliding cycles. The coefficient of friction was found to decrease with the number of repeats, and after 20 repeats the coefficient of friction reaches a constant value. Several authors observed the transfer of lipid material from the finger to the sample surface $[12,13,55]$. Multiple use of test samples demands a thorough cleaning to remove this transfer layer, as it influences the friction between the two surfaces. This is achieved by cleaning all samples ultrasonically using ethanol before and in between the experiments.

It can be concluded that there are many causes of variations in the observed friction behaviour. Many of these are intrinsic to the tactile exploration of product surfaces. This thesis focuses on the role of the 


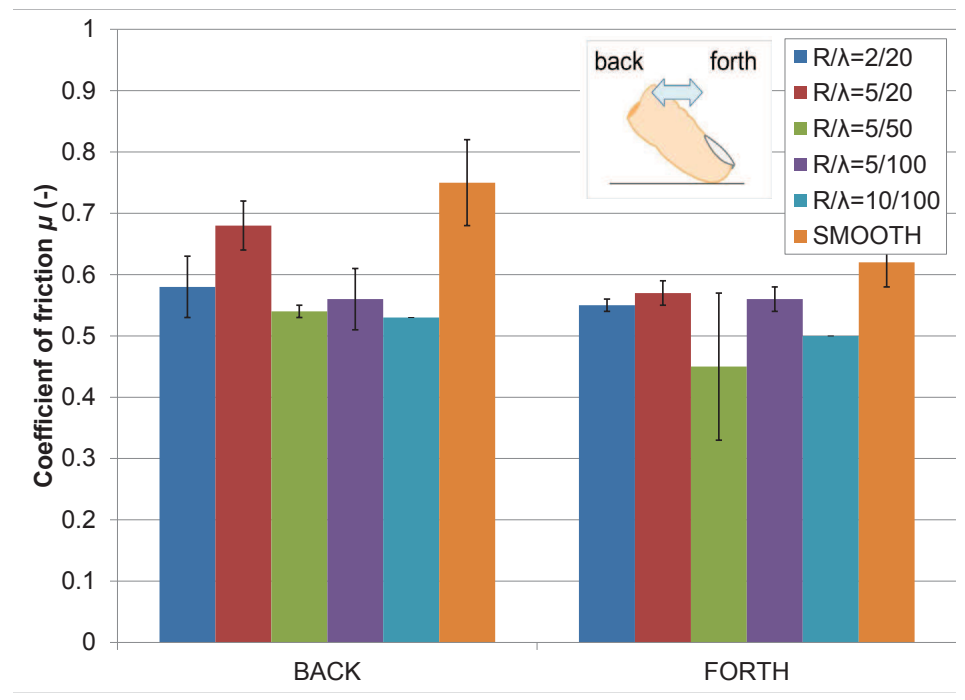

Figure 4.6: Coefficient of friction of the index finger for sliding directions back and forth.

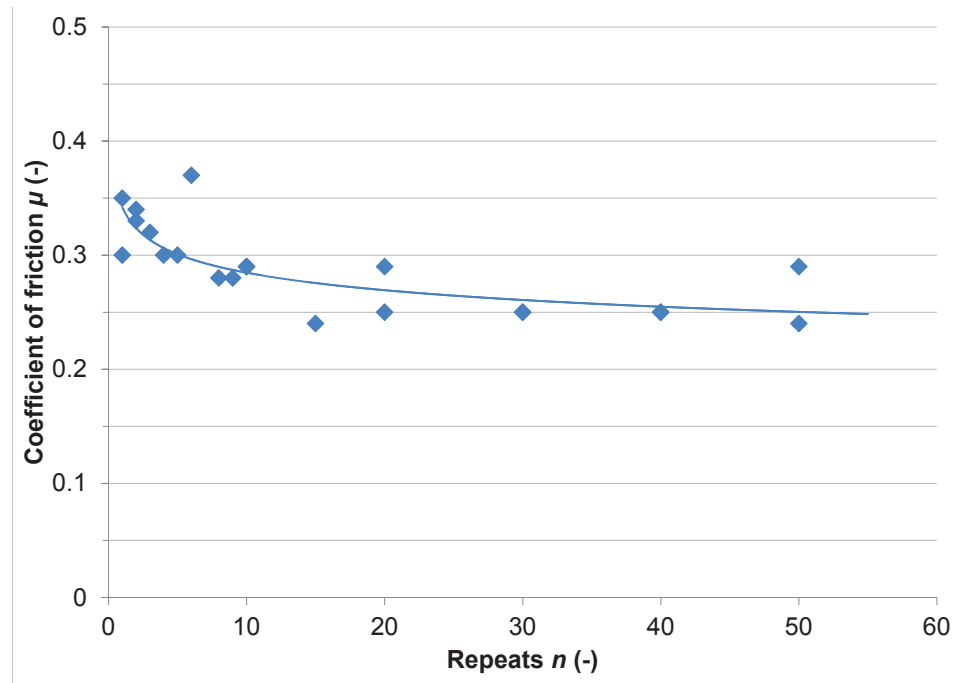

Figure 4.7: Coefficient of friction between the normal skin of the index finger and a textured sample at $1 \mathrm{~N}$ normal load as a function of the number of repeats. 
product surface properties, such as the surface microgeometry, in the friction behaviour.

\subsection{Experiments using textured surfaces}

In vivo experiments were carried out by sliding the fingerpad of the left (dominant) index finger of a 38-year-old male subject against surfaces with regular surface textures. The standard deviation of the measurements carried out between the textured samples and dry skin varies between approximately 10 and 20\%. The average coefficient of friction was obtained from measurements involving the left index finger of a single subject. The only planned variation in test conditions was the time and day: measurements were carried out on different days within a period of two months, at different times of the day. The average hydration level measured during the test program is $42 \mathrm{AU}$, with a minimum of $34 \mathrm{AU}$ and a maximum of $49 \mathrm{AU}$. Even though this range is considered too small to be of influence, closer observation of the results shows that there is no correlation between the measured hydration level and the friction of skin in its normal state. Analysis of the measurement data revealed that the variation of the normal load is small. The average normal load is $1.23 \mathrm{~N}$ with $21 \%$ standard deviation. When taking into account the minor effect the normal load has on the friction within the range 1 to $2 \mathrm{~N}$, the influence of variation of normal load on the measured friction is considered to be negligible within this load range. In this study the intended orientation of the fingerpad is approximately $30^{\circ}$. However, in practice the angle varies between $20^{\circ}$ and $40^{\circ}$, so that the orientation of the fingerpad is expected to contribute considerably to the observed variation in test results.

Figure 4.8 shows the coefficient of friction measured against textured samples as a function of tip spacing and normal load. With the tip spacing increasing from 20 to 50 and $100 \mu \mathrm{m}$, at $1 \mathrm{~N}$ normal load the coefficient of friction, averaged over five measurements, decreases from 0.48 to 0.38 and 0.36 respectively. Since this corresponds to the trend predicted by equation (3.7), it can be concluded that adhesive friction is the dominant mechanism especially at small spacing. At larger spacing the deformation component of friction becomes more important so that the trend of a decreasing coefficient 




Figure 4.8: Coefficient of friction as a function of tip spacing for the normal skin of the index finger at $1 \mathrm{~N}$ normal load and $\sim 30^{\circ}$ orientation: mean values and standard deviations.

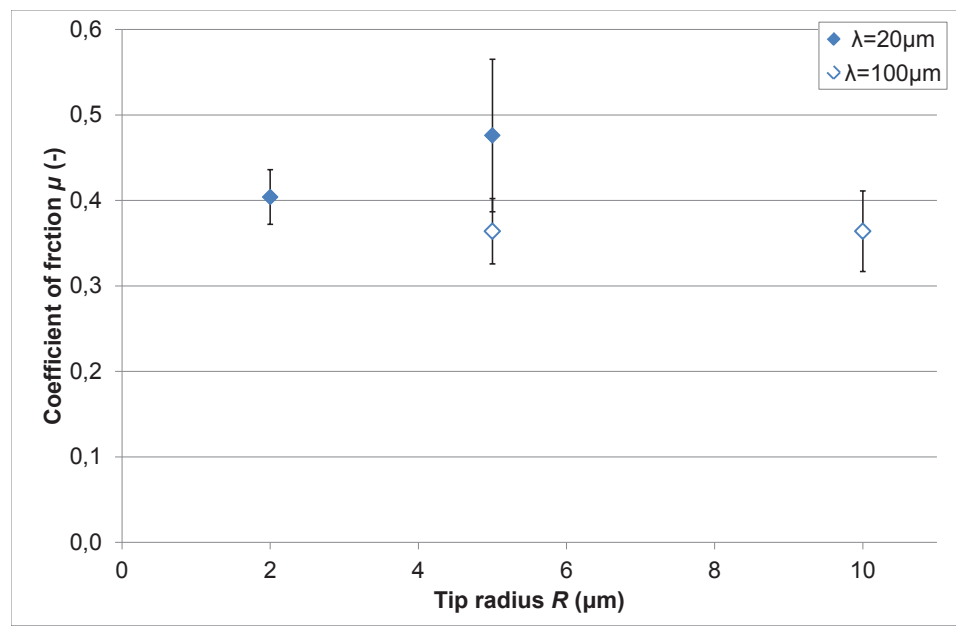

Figure 4.9: Coefficient of friction as a function of tip radius for the normal skin of the index finger at $1 \mathrm{~N}$ normal load and $\sim 30^{\circ}$ orientation: mean values and standard deviations 
of friction becomes weaker. Adhesive friction being the dominant friction mechanism suggests a clear dependence of the coefficient of friction on tip radius as described by Equation (3.7).

Figure 4.9 shows the coefficient of friction measured against textured samples as a function of tip radius of curvature and normal load. At $1 \mathrm{~N}$ normal load and $20 \mu \mathrm{m}$ tip spacing the average coefficient of friction increases from 0.4 to 0.48 when the tip radius increases from 2 to $5 \mu \mathrm{m}$. At $100 \mu \mathrm{m}$ tip spacing the average coefficient of friction remains constant at 0.36 when the tip radius increases from 5 to $10 \mu \mathrm{m}$.

Since the number of contacts is high when the distance between the tips is small, the applied load on each individual asperity is low. Therefore, the normal adhesion between the skin and the countersurface contributes considerably to the actual load. The contribution of normal adhesion increases with increasing tip radius, hence the higher coefficient of friction. Different mechanisms determine the magnitude of the coefficient of friction for these regular surface geometries. At larger tip spacings the contribution of normal adhesion is not as important as it is at smaller tip spacings. Due to the larger tip spacing, the deformation component of friction comes into play. The effect of tip radius on the different mechanisms, adhesive and deformation friction is reversed as shown in Equations (3.7) and (3.8). The relative contributions of the different friction mechanisms are sensitive to differences in test conditions such as the state of the skin.

Figs. 4.8 and 4.9 show that the variation in results is the largest when, following the theory developed earlier, adhesion has the most influence. It is common to describe the frictional properties of a surface by the coefficient of friction which is obtained by dividing the measured friction force by the applied normal load following Equation (2.1). However, what is measured is the friction force which results from the real normal load, which is the applied load plus the adhesion force between the two surfaces

$$
\begin{aligned}
F_{\mu} & =\mu_{\text {real }} \cdot F_{\text {real }} \\
& =\mu_{\text {real }} \cdot\left(F_{n}+F_{a d h}\right)
\end{aligned}
$$

When the measured coefficient of friction $\mu$ is calculated by using equation 
(2.1), what is actually calculated is

$$
\mu=\mu_{\text {real }} \cdot\left(1+\frac{F_{a d h}}{F_{n}}\right)
$$

Equation (4.3) shows that the effect of adhesion becomes important when the normal load is of the same order of magnitude as the adhesion force. For example, for a textured sample with $5 \mu \mathrm{m}$ radius asperities at $20 \mu \mathrm{m}$ spacing, at $1 \mathrm{~N}$ normal load the total adhesion force $F_{a d h}$ varies between 0 and $0.3 \mathrm{~N}$, depending on the surface condition of the skin. So under conditions where adhesion plays a role, variations in hydration level and cleanliness of the skin and the sample surface will result in considerable variations in the adhesion force and thus in variations in the observed friction.

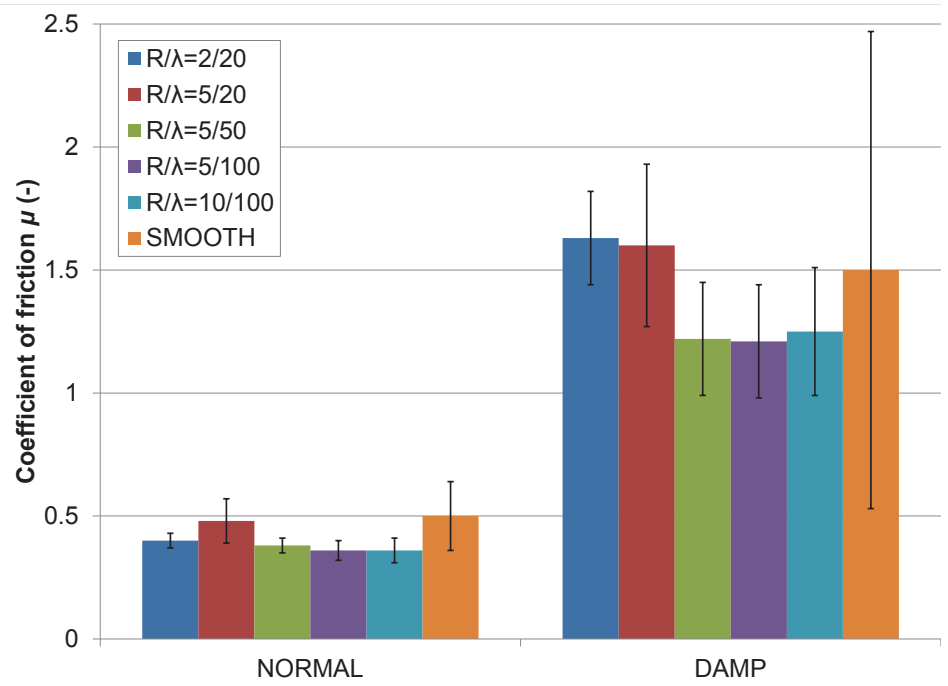

Figure 4.10: Coefficient of friction for normal and damp skin of the index finger at $1 \mathrm{~N}$ normal load and $\sim 30^{\circ}$ orientation for different texture parameters.

Figure 4.10 shows the coefficient of friction measured against textured samples for normal and damp skin. The average hydration level during the tests was $42 \mathrm{AU}$ for normal skin, within the range 34-50 AU, and $97 \mathrm{AU}$ for damp skin, within the range 77-108 AU. The coefficient of friction measured against damp skin is considerably larger than against normal, or dry, skin. For damp skin the coefficient of friction decreases from about 1.6 at $20 \mu \mathrm{m}$ 
tip spacing to about 1.2 at 50 and $100 \mu \mathrm{m}$ and is independent of tip radius. The increase is more than threefold when compared to the skin in its normal state. From the friction results depicted in Figs. 4.8 and 4.9, the viscoelastic loss modulus $\beta$ and surface shear strength $\tau$ can be approximated using Equations (3.7) and (3.8). Yuan and Verma measured the viscoelastic loss modulus $\beta$ from dynamic indentation experiments finding a value of 0.31 for dry and 0.79 for wet skin [10]. Since the skin of the fingerpad is slightly hydrated as shown earlier, a value for $\beta=0.55$ was used in the calculations. A surface shear strength varying between 1.4 and $4.5 \mathrm{MPa}$ is calculated from the measured friction coefficients, which is comparable to values reported by e.g. Persson et al. [56].

\subsection{Experiments using sandpaper}

A sample material commonly used to investigate perceived roughness is sandpaper, see for instance $[57,58]$. Furthermore, a positive correlation was observed between perceived roughness and the measured coefficient of friction [59]. Friction experiments were carried out using different grades of sandpaper. 3M Wetordry Tri-M-ite abrasive paper was used in different grades between $\mathrm{p} 80$ and p2000, so that average particle diameters varied from 201 down to $10.3 \mu \mathrm{m}$. The silicon carbide grit is bonded with a waterproof resin and covers $100 \%$ of the closed coat paper.

Figure 4.11 shows the coefficient of friction between the fingerpad and sandpaper for normal and damp skin. For normal skin, friction increases with increasing particle diameter from an approximate coefficient of friction of 0.93 at a diameter of $10.3 \mu \mathrm{m}$ to 1.48 at a particle diameter of $201 \mu \mathrm{m}$. For damp skin, the coefficient of friction decreases with increasing particle diameter from about 1.62 at $10.3 \mu \mathrm{m}$ to 1.15 at $201 \mu \mathrm{m}$.

With increasing particle diameter the density of the abrasive particles decreases, which increases the load on each individual particle. It was shown earlier that when the load on an individual asperity increases, deformation becomes a more important friction mechanism. Increased ploughing explains the increase of the coefficient of friction with particle diameter when sliding against dry skin.

The decreasing friction observed with damp skin is caused by the 


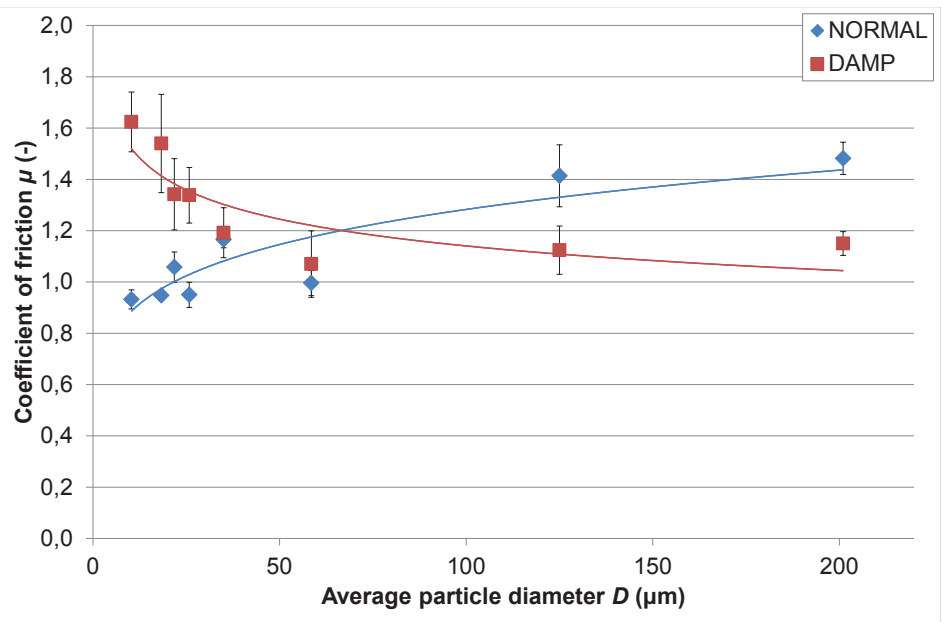

Figure 4.11: Coefficient of friction measured against sandpaper as a function of particle diameter for normal and damp skin of the index finger at $1 \mathrm{~N}$ normal load and $\sim 30^{\circ}$ orientation: mean values and standard deviations

dependence of the mechanical properties on the hydration level of the stratum corneum, the top layer of the skin. Softening of the stratum corneum leads to an increased contact area and increased indentation depth. The adhesive component of the friction force increases, especially at small particle diameters, where the density of the particles is high. Whereas for normal skin the deformation component is negligible at small particle diameters, for damp skin deformation friction does play a role.

Although Fig. 4.11 plots the coefficient of friction as a function of particle diameter, friction is also determined by other factors, such as the shape and the particle density. Since these properties do not necessarily correlate, the relation between particle diameter and the coefficient of friction plotted in Fig. 4.11 does not follow a smooth line. 


\section{Chapter 5}

\section{Conclusions and \\ Recommendations}

In this thesis a model was developed for the contact and tactile friction behaviour of human skin as a function of asperity geometry and operational conditions. The mechanistic approach adopted in this research (summarized in Paper F) has resulted in the conclusions which are presented per chapter in the following section. Finally, some recommendations for future research are given.

\subsection{Conclusions}

\section{Contact and friction of skin}

- The contact behaviour of skin can be described accurately by a single parameter: the effective elastic modulus. For contact modelling involving relatively small deformations, the complex behaviour of the skin of the volar forearm is described using a closed-form expression $E=f(a)$ giving the elastic modulus $E$ as a function of contact length scale $a$.

- The effective elastic modulus decreases several orders of magnitude with increasing length scale, from about $1 \mathrm{MPa}$ at a length scale of 1 $\mu \mathrm{m}$ to several $\mathrm{kPa}$ at the millimeter scale. 
- Analytical models from contact mechanics theory were used to develop a model able to predict the contact of human skin. To incorporate the influence of the skin microrelief of the non-glabrous skin, in this thesis a multiscale contact model was developed in which the surface topography of the skin is considered as a nominal flat surface having a profile characterized by a wavelength and amplitude.

- Incorporation of the contact model into the two-term friction model enables the prediction of the friction behaviour of a spherical smooth probe sliding against the skin of the inner forearm.

\section{Modelling tactile friction}

- The model developed in this thesis can be used to estimate the friction behaviour of the human fingerpad as a function of asperity geometry and operational conditions. The developed model explains the mechanisms which determine the coefficient of friction.

- For textures consisting of evenly distributed spherically-tipped asperities:

- Friction increases with increasing asperity tip radius because of increasing normal adhesion.

- The two-term friction model shows that at high asperity density adhesive friction is the dominant mechanism and friction increases with increasing asperity density. At low asperity density deformation friction becomes more important and friction decreases with increasing asperity density.

\section{Measuring tactile friction}

- The variation of the friction observed in the tactile friction experiments described in this thesis can be assigned to differences in contacting area which are caused by a combination of orientation of the finger and pressing force.

- Under conditions where normal adhesion plays an important role, such as with smooth surfaces or textures with high asperity densities, 
measured friction changes with hydration level and cleanliness of the skin leading to a large variation in observed results.

- When carrying out a tactile friction measurement a well-defined experimental procedure should be followed:

- It is recommended to measure the frictional behaviour of all the fingers, especially when the objective is to relate the measured friction properties to the perception of slipperiness or smoothness.

- The samples should be thoroughly cleaned to assure high levels of repeatability. The measurements should be carried out under constant environmental conditions.

- The friction measurement should comprise the measurement of the tangential friction force as well as the normal load on the contact. For low loads the use of dead weights attached to the fingerpad is preferred. The hydration level of the skin should be monitored during the test program.

- It is important to acknowledge that variations in the orientation angle and pressing force, and changes in skin condition, lead to considerable changes in the measured coefficient of friction.

\subsection{Recommendations for future research}

- The contact and friction model developed in this thesis explains the tribological phenomena occurring at the interface between the human skin and a product surface. To understand 'product feel' the model should be extended to predict the stresses and strains at the mechanoreceptor locations underneath the skin surface.

- The developed contact model is valid for model surfaces, i.e. regular textures, and should be extended to engineering surfaces, i.e. random surface topographies, taking into account interaction between asperities.

- The developed model has been validated against surface textures applied on steel, a material which is rigid as compared to human skin. 
To apply the model to a larger range of product surfaces, such as 'soft-touch' elastomers, deformation of the countersurface should be taken into account.

- The two-term friction model is capable of predicting the friction behaviour of the human fingerpad. Further optimization requires an improved understanding of the chemical and mechanical aspects that determine the interfacial shear strength at the scale of the contacting summits. 


\section{Bibliography}

[1] E. Karana, P. Hekkert, and P. Kandachar. Meanings of materials through sensorial properties and manufacturing processes. Materials \& Design, 30(7):2778-2784, 2009.

[2] C.J. Barnes, T.H.C. Childs, B. Henson, and C.H. Southee. Surface finish and touch - a case study in a new human factors tribology. Wear, 257(7-8):740-750, 2004.

[3] T.H.C. Childs and B. Henson. Human tactile perception of screen-printed surfaces: Self-report and contact mechanics experiments. Proceedings of the Institution of Mechanical Engineers, Part J: Journal of Engineering Tribology, 221(3):427-441, 2007.

[4] X. Chen, C.J. Barnes, T.H.C. Childs, B. Henson, and F. Shao. Materials' tactile testing and characterisation for consumer products' affective packaging design. Materials \&5 Design, 30(10):4299-4310, 2009.

[5] M.A. Darden and C.J. Schwartz. Investigation of skin tribology and its effects on the tactile attributes of polymer fabrics. Wear, 267(5-8):1289-1294, 2009.

[6] L. Skedung, K. Danerlöv, U. Olofsson, C.M. Johannesson, M. Aikala, J. Kettle, M. Arvidsson, B. Berglund, and M.W. Rutland. Tactile perception: Finger friction, surface roughness and perceived coarseness. Tribology International, 44(5):505-512, 2011.

[7] S. Schreiner, M. Rechberger, and J. Bertling. Haptic perception of friction: correlating friction measurements of skin against polymer surfaces with subjective evaluations of the surfaces' grip. Tribology International, 63:21-28, 2013. 
[8] H. Czichos, editor. Tribology: A systems approach to the science and technology of friction, lubrication and wear. Elsevier, 1978.

[9] D.J. Tobin. Biochemistry of human skin - our brain on the outside. Chemical Society Reviews, 35(1):52-67, 2006.

[10] Y. Yuan and R. Verma. Measuring microelastic properties of stratum corneum. Colloids and Surfaces B: Biointerfaces, 48(1):6-12, 2006.

[11] C. Pailler-Mattéi, S. Pavan, R. Vargiolu, F. Pirot, F. Falson, and H. Zahouani. Contribution of stratum corneum in determining bio-tribological properties of the human skin. Wear, 263(7-12):1038-1043, 2007.

[12] R.T. Spurr. Fingertip friction. Wear, 39(1):167-171, 1976.

[13] O.S. Dinç, C.M. Ettles, S.J. Calabrese, and H.A. Scarton. Some parameters affecting tactile friction. Journal of Tribology, 113(3):512-517, 1991.

[14] S.A. Johnson, D.M. Gorman, M.J. Adams, and B.J. Briscoe. The friction and lubrication of human stratum corneum. In C.M. Taylor D. Dowson, editor, Thin Films in Tribology, Proceedings of the 19th Leeds-Lyon Symposium on Tribology held at the Institute of Tribology, University of Leeds, volume 25, pages 663-672. Elsevier, 1993.

[15] M.J. Adams, B.J. Briscoe, and S.A. Johnson. Friction and lubrication of human skin. Tribology Letters, 26(3):239-253, 2007.

[16] H. Hertz. Über die Berührung fester elastischer Körper. Journal für die reine und angewandte Mathematik, 92:156-171, 1882.

[17] P.F.D. Naylor. The skin surface and friction. British Journal of Dermatology, 67(7):239-248, 1955.

[18] A.F. El-Shimi. In vivo skin friction measurements. Journal of the Society of Cosmetic Chemists, 28(2):37-52, 1977.

[19] J. Asserin, H. Zahouani, Ph. Humbert, V. Couturaud, and D. Mougin. Measurement of the friction coefficient of the human skin in vivo: 
Quantification of the cutaneous smoothness. Colloids and Surfaces B: Biointerfaces, 19(1):1-12, 2000.

[20] R. K. Sivamani, J. Goodman, N.V. Gitis, and H.I. Maibach. Friction coefficient of skin in real-time. Skin Research and Technology, 9(3):235-239, 2003.

[21] A.A. Koudine, M. Barquins, Ph. Anthoine, L. Aubert, and J.-L. Lèvêque. Frictional properties of skin: proposal of a new approach. International Journal of Cosmetic Science, 22(1):11-20, 2000.

[22] K. Elleuch, R. Elleuch, and H. Zahouani. Comparison of elastic and tactile behavior of human skin and elastomeric materials through tribological tests. Polymer Engineering \& Science, 46(12):1715-1720, 2006.

[23] W. Li, M. Kong, X.D. Liu, and Z.R. Zhou. Tribological behavior of scar skin and prosthetic skin in vivo. Tribology International, 41(7):640-647, 2008 .

[24] W. Tang, S.-R. Ge, H. Zhu, X.-C. Cao, and N. Li. The influence of normal load and sliding speed on frictional properties of skin. Journal of Bionic Engineering, 5(1):33-38, 2008.

[25] H. Zahouani, R. Vargiolu, G. Boyer, C. Pailler-Mattei, L. Laquièze, and A. Mavon. Friction noise of human skin in vivo. Wear, 267(5-8):1274-1280, 2009.

[26] J. Jachowicz, R. McMullen, and D. Prettypaul. Indentometric analysis of in vivo skin and comparison with artificial skin models. Skin Research and Technology, 13(3):299-309, 2007.

[27] K. L. Johnson. Contact mechanics. Cambridge University Press, Cambridge, 1999.

[28] S. Derler and L.-C. Gerhardt. Tribology of skin: Review and analysis of experimental results for the friction coefficient of human skin. Tribology Letters, 45:1-27, 2011. 
[29] M. Geerligs, L. van Breemen, G. Peters, P. Ackermans, F. Baaijens, and C. Oomens. In vitro indentation to determine the mechanical properties of epidermis. Journal of Biomechanics, 44(6):1176-1181, 2011.

[30] R.H. Wildnauer, J.W. Bothwell, and A.B. Douglass. Stratum corneum biomechanical properties I. Influence of relative humidity on normal and extracted human stratum corneum. J Investig Dermatol, 56(1):72-78, 1971.

[31] A.C. Park and C.B. Baddiel. Rheology of stratum corneum - i: A molecular interpretation of the stress-strain curve. J Soc Cosmet Chem, 23:3-12, 1972.

[32] Y.S. Papir, K.-H. Hsu, and R.H. Wildnauer. The mechanical properties of stratum corneum: I. The effect of water and ambient temperature on the tensile properties of newborn rat stratum corneum. Biochimica et Biophysica Acta (BBA) - General Subjects, 399(1):170-180, 1975.

[33] A. Delalleau, G. Josse, J.-M. Lagarde, H. Zahouani, and J.-M. Bergheau. Characterization of the mechanical properties of skin by inverse analysis combined with the indentation test. Journal of Biomechanics, 39(9):1603-1610, 2006.

[34] C. Pailler-Mattéi, S. Nicoli, F. Pirot, R. Vargiolu, and H. Zahouani. A new approach to describe the skin surface physical properties in vivo. Colloids and Surfaces B: Biointerfaces, 68(2):200-206, 2009.

[35] H. Zahouani, C. Pailler-Mattei, B. Sohm, R. Vargiolu, V. Cenizo, and R. Debret. Characterization of the mechanical properties of a dermal equivalent compared with human skin in vivo by indentation and static friction tests. Skin Research and Technology, 15(1):68-76, 2009.

[36] S. Bec, A. Tonck, J.-M. Georges, E. Georges, and J.-L. Loubet. Improvements in the indentation method with a surface force apparatus. Philosophical Magazine A, 74(5):1061-1072, 1996.

[37] C. Pailler-Mattéi, S. Bec, and H. Zahouani. In vivo measurements of the elastic mechanical properties of human skin by indentation tests. Medical Engineering \& Physics, 30(5):599-606, 2008. 
[38] C. Pailler-Mattéi and H. Zahouani. Study of adhesion forces and mechanical properties of human skin in vivo. Journal of Adhesion Science and Technology, 18:1739-1758, 2004.

[39] J.F. Archard. Elastic deformation and the laws of friction. Proceedings of the Royal Society of London. Series A. Mathematical and Physical Sciences, 243(1233):190-205, 1957.

[40] J.M. Lagarde, C. Rouvrais, and D. Black. Topography and anisotropy of the skin surface with ageing. Skin Research and Technology, 11(2):110-119, 2005.

[41] G. Boyer, L. Laquièze, A. Le Bot, S. Laquièze, and H. Zahouani. Dynamic indentation on human skin in vivo: ageing effects. Skin Research and Technology, 15(1):55-67, 2009.

[42] U. Jacobi, M. Chen, G. Frankowski, R. Sinkgraven, M. Hund, B. Rzany, W. Sterry, and J. Lademann. In vivo determination of skin surface topography using an optical 3D device. Skin Research and Technology, 10(4):207-214, 2004.

[43] C. Pailler-Mattéi and H. Zahouani. Analysis of adhesive behaviour of human skin in vivo by an indentation test. Tribology International, 39(1):12-21, 2006.

[44] K.L. Johnson, K. Kendall, and A.D. Roberts. Surface energy and the contact of elastic solids. Proceedings of the Royal Society of London. A. Mathematical and Physical Sciences, 324(1558):301-313, 1971.

[45] J. Škvarla. Hydrophobic interaction between macroscopic and microscopic surfaces. Unification using surface thermodynamics. Advances in Colloid and Interface Science, 91(3):335-390, 2001.

[46] C. Flynn, A. Taberner, and P. Nielsen. Measurement of the force-displacement response of in vivo human skin under a rich set of deformations. Medical Engineering \&f Physics, 33(5):610-619, 2011.

[47] M. Tomimoto. The frictional pattern of tactile sensations in anthropomorphic fingertip. Tribology International, 44(11):1340-1347, 2011. 
[48] P.H. Warman and A.R. Ennos. Fingerprints are unlikely to increase the friction of primate fingerpads. Journal of Experimental Biology, 212(13):2016-2022, 2009.

[49] M.M. Taylor and S.J. Lederman. Tactile roughness of grooved surfaces: A model and the effect of friction. Perception 8 Psychophysics, 17(1):23-36, 1975.

[50] T. Maeno, K. Kobayashi, and N. Yamazaki. Relationship between the structure of human finger tissue and the location of tactile receptors. JSME International Journal Series C, 41:94-100, 1998.

[51] A.B. Cua, K.P. Wilhelm, and H.I. Maibach. Frictional properties of human skin: relation to age, sex and anatomical region, stratum corneum hydration and transepidermal water loss. British Journal of Dermatology, 123(4):473-479, 1990.

[52] L.-C Gerhardt, V. Strässle, A. Lenz, N. D. Spencer, and S. Derler. Influence of epidermal hydration on the friction of human skin against textiles. Journal of The Royal Society Interface, 5(28):1317-1328, 2008.

[53] M. Kwiatkowska, S. E. Franklin, C. P. Hendriks, and K. Kwiatkowski. Friction and deformation behaviour of human skin. Wear, 267(5-8):1264-1273, 2009.

[54] N.K. Veijgen, M.A. Masen, and E. van der Heide. Relating friction on the human skin to the hydration and temperature of the skin. Tribology Letters, 49(1):251-262, 2013.

[55] L. Skedung, K. Danerlöv, U. Olofsson, M. Aikala, K. Niemi, J. Kettle, and M.W. Rutland. Finger friction measurements on coated and uncoated printing papers. Tribology Letters, 37(2):389-399, 2010.

[56] B.N.J. Persson, A. Kovalev, and S.N. Gorb. Contact mechanics and friction on dry and wet human skin. Tribology Letters, 50(1):17-30, 2013.

[57] S.S. Stevens and J.R. Harris. The scaling of subjective roughness and smoothness. Journal of Experimental Psychology, 64(5):489-494, 1962. 
[58] R.T. Verrillo, S.J. Bolanowski, C.M. Checkosky, and F.P. McGlone. Effects of hydration on tactile sensation. Somatosensory \& Motor Research, 15(2):93-108, 1998.

[59] W.M. Bergmann Tiest. Tactual perception of material properties. Vision Research, 50(24):2775-2782, 2010. 



\section{Part II}

Paper A: J. van Kuilenburg, M.A. Masen and E. van der Heide. A review of fingerpad contact mechanics and friction and how this affects tactile perception. Proceedings of the IMechE Part J: Journal of Engineering Tribology, accepted for publication.

Paper B: J. van Kuilenburg, M.A. Masen and E. van der Heide. Contact modelling of human skin: What value to use for the modulus of elasticity? Proceedings of the IMechE Part J: Journal of Engineering Tribology, 227(4):347-359, 2013.

Paper C: J. van Kuilenburg, M.A. Masen and E. van der Heide. The role of the skin microrelief in the contact behaviour of human skin: Contact between the human finger and regular surface textures. Tribology International, 65:81-90, 2013.

Paper D: J. van Kuilenburg, M.A. Masen, M.N.W. Groenendijk, V. Bana and E. van der Heide. An experimental study on the relation between surface texture and tactile friction. Tribology International, 48:15-21, 2012.

Paper E: J. van Kuilenburg, M.A. Masen and E. van der Heide. Touch properties of surfaces: How to measure tactile friction in vivo. Materials $\&$ Design, submitted.

Paper F: J. van Kuilenburg, M.A. Masen and E. van der Heide. A mechanistic approach to predicting the friction behaviour of human skin. In Proceedings of the ASME/STLE 2012 International Joint Tribology Conference, Denver, USA, 2012. 



\section{Paper A}

\section{A review of fingerpad contact mechanics and friction}

and how this affects tactile perception

J. van Kuilenburg, M.A. Masen and E. van der Heide

Accepted for publication in

Proceedings of the IMechE Part J: Journal of Engineering Tribology

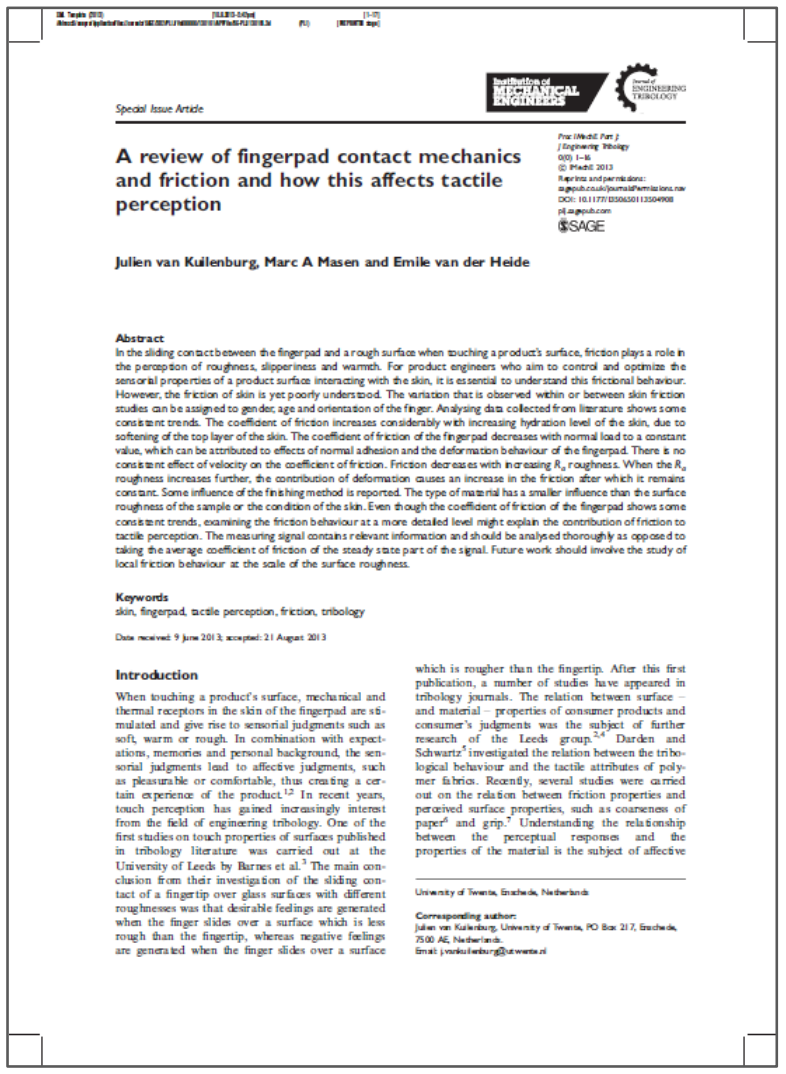





\title{
A review of fingerpad contact mechanics and friction and how this affects tactile perception
}

\author{
Julien van Kuilenburg, Marc A. Masen and Emile van der Heide
}

\begin{abstract}
In the sliding contact between the fingerpad and a rough surface when touching a product's surface, friction plays a role in the perception of roughness, slipperiness and warmth. For product engineers who aim to control and optimize the sensorial properties of a product surface interacting with the skin, it is essential to understand this frictional behaviour. However, the friction of skin is yet poorly understood. The variation that is observed within or between skin friction studies can be assigned to gender, age and orientation of the finger. Analyzing data collected from the literature shows some consistent trends. The coefficient of friction increases considerably with an increasing hydration level of the skin, due to softening of the top layer of the skin. The coefficient of friction of the fingerpad decreases with normal load to a constant value, which can be attributed to effects of normal adhesion and the deformation behaviour of the fingerpad. There is no consistent effect of velocity on the coefficient of friction. Friction decreases with increasing $R_{a}$ roughness. When the $R_{a}$ roughness increases further, the contribution of deformation causes an increase in the friction, after which it remains constant. Some influence of the finishing method is reported. The type of material has a smaller influence than the surface roughness of the sample or the condition of the skin. Even though the coefficient of friction of the fingerpad shows some consistent trends, examining the friction behaviour at a more detailed level might explain the contribution of friction to tactile perception. The measuring signal contains relevant information and should be analyzed thoroughly, as opposed to taking the average coefficient of friction of the steady-state part of the signal. Future work should involve the study of local friction behaviour at the scale of the surface roughness.
\end{abstract}

\section{Keywords}

skin, fingerpad, tactile perception, friction, tribology 


\section{Introduction}

When touching a product's surface, mechanical and thermal receptors in the skin of the fingerpad are stimulated and give rise to sensorial judgments such as soft, warm or rough. In combination with expectations, memories and personal background, the sensorial judgments lead to affective judgments, such as pleasurable or comfortable, thus creating a certain experience of the product $[1,2]$. In recent years, engineering tribology has increasingly shown interest in touch perception. One of the first studies on touch properties of surfaces published in tribology literature was carried out at the University of Leeds by Barnes et al. [3]. The main conclusion from their investigation of the sliding contact of a fingertip over glass surfaces with different roughnesses was that desirable feelings are generated when the finger slides over a surface which is less rough than the fingertip, whereas negative feelings are generated when the finger slides over a surface which is rougher than the fingertip. Following this first publication a number of studies have appeared in tribology journals. The relation between surface - and material - properties of consumer products and consumer's judgments was the subject of further research by the Leeds group [2, 4]. Darden and Schwartz investigated the relation between the tribological behaviour and the tactile attributes of polymer fabrics [5]. Recently, several studies were carried out on the relation between friction properties and perceived surface properties, such as coarseness of paper [6] and grip [7]. Understanding the relationship between the perceptual responses and the properties of the material is the subject of affective engineering. From the viewpoint of the tribologist it is the relation between the surface properties of the material and the sensorial and affective responses that is of most interest. The sensorial responses, also psychophysical responses, can be related almost directly to physical measurements of material and surface properties. Perceptions of roughness, warmth, softness and slipperiness can be related to measures of surface roughness, thermal conductivity, compliance and friction [8] using statistical techniques.

The senses of roughness, softness and slipperiness are mediated through mechanoreceptive afferents located in the skin. The different types of mechanoreceptors respond to spatio-temporal stimuli such as strains, or changes therein, and vibrations. This is where contact mechanics and, in the case of sliding motion between the finger and a surface, the friction between the surfaces comes in. For instance, in a sliding contact the maximum tensile stress at the surface and the maximum shear stress beneath the surface are proportional to the coefficient of friction [9]. The coefficient of friction $\mu$, which is calculated as the quotient of the measured friction force $F_{\mu}$ and the applied normal load $F$, is commonly 
used in mechanical engineering to describe the friction behaviour of a system.

$$
\mu=\frac{F_{\mu}}{F}
$$

Numerous studies have reported on the friction behaviour of the human fingerpad. In their review paper, Derler and Gerhardt [10] present a wide range of coefficients of friction as a function of countersurface material, surface roughness, normal load and skin condition. They further conclude that tactile perception and touch in relation to skin tribology is largely understudied and poorly understood. This paper aims at building an understanding of tactility and tactile friction from an engineering tribology background.

\section{Tactile perception}

Understanding product feel and the interaction of human skin and product surfaces requires a thorough knowledge of the tribological phenomena occurring at the interface between the human skin and the product surface. On the other hand, touch perception, which for the larger part starts with the stimulation of mechanoreceptors beneath the skin surface, can be explained from a mechanical point of view. When sliding the fingerpad over a surface, the characteristics of the surface and the material beneath will stimulate the skin senses, leading to sensorial judgments of softness, warmth, roughness and slipperiness. Figure 1 summarizes the physical stimuli and the psychophysical sensations in a schematical way.

\section{Softness}

In engineering, hardness refers to indentation hardness, which is basically the resistance of the surface and the underlying material against plastic deformation. In psychophysics, the 'softness' of an object is related to the compliance, or the lack of stiffness. The compliance can be expressed in terms of stiffness $k$, which gives the relation between the applied force and the resulting deformation, or by the elastic modulus $E$, which describes the relation between the applied stresses and the resulting strains. Obviously, stiffness and elastic modulus are related. Whereas the elastic modulus is a material property, the stiffness is a property of the object, also depending on the geometry. Whether the stiffness or the elastic modulus is the representative parameter of an object's softness depends on how soft or hard the object is perceived: it has been found that for the softer stimuli the judgment of subjects is based on force-displacement information, thus stiffness, whereas for the harder stimuli the judgment is based on surface information, which is determined by the elastic modulus [8]. From a physiological point of view this can be explained by the fact that part 


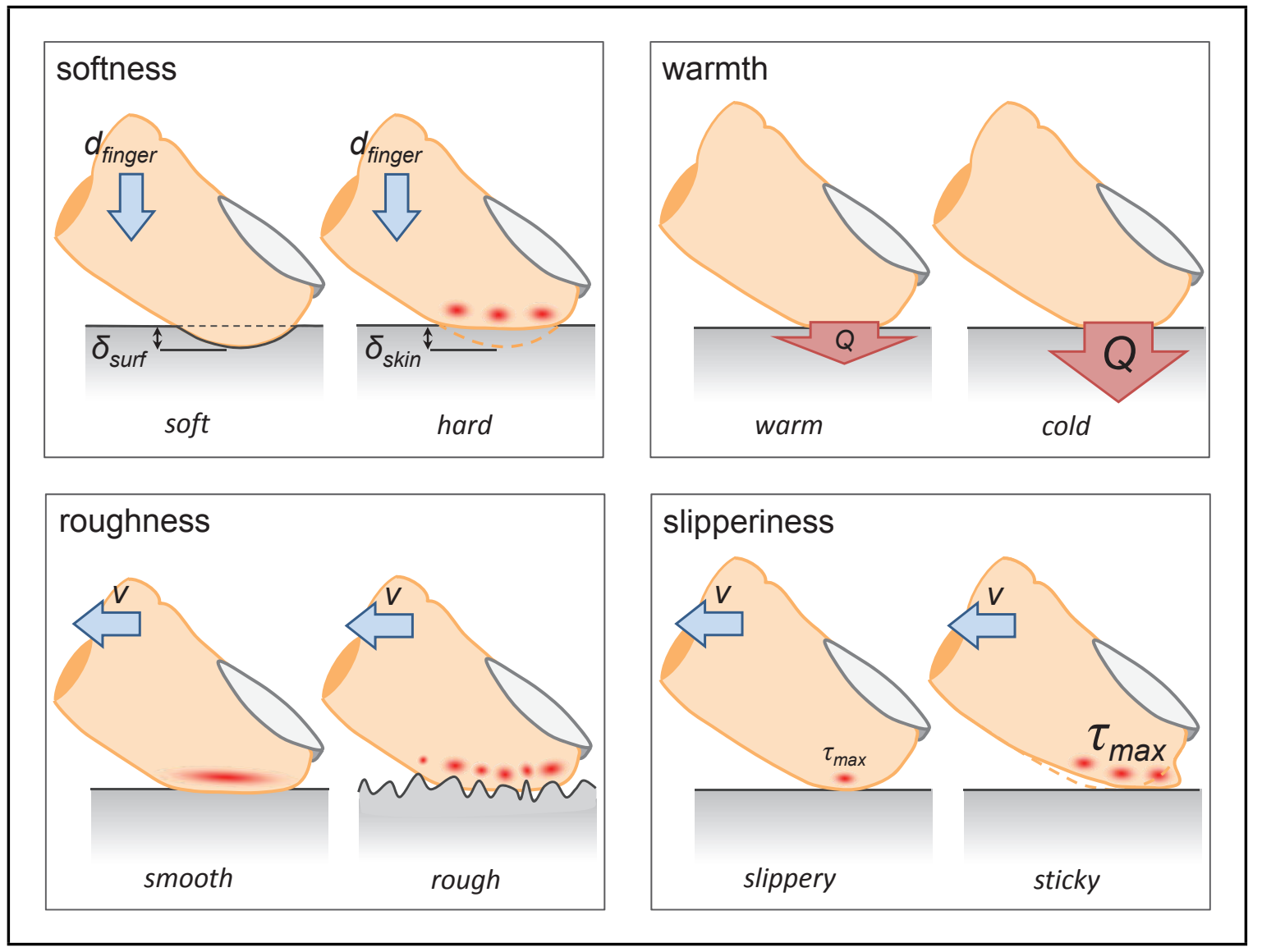

Figure 1. Schematic overview of the sensorial properties softness, warmth, roughness and slipperiness and used definitions. 'Soft' and 'hard' relate to surface and skin deformation; 'warm' and 'cold' to small and large heat flux out of the skin; 'smooth' and 'rough' to a uniform or irregular pressure distribution; 'slippery' and 'sticky' to small and large strains in the skin. 
of the information comes from cutaneous stimuli caused by surface deformation, and part of the information comes kinaesthetic stimuli combining force and displacement [8].

In mechanical terms: from the point where the finger touches a surface and starts pressing, the relation between the applied force $F$ and resulting displacement $d_{\text {finger }}$ can be described as

$$
F=k^{*} d_{\text {finger }}
$$

where the reduced stiffness $k^{*}$ of the contact can be defined as the product of the diameter of the contact $2 a$ and reduced elastic modulus $E^{*}$

$$
k^{*}=2 a E^{*}
$$

The displacement of the fingerpad, the kinaesthetic stimulus, is composed of the deformation of the object $\delta_{\text {surf }}$ and the deformation of the fingerpad $\delta_{\text {skin }}$, the cutaneous stimulus

$$
d_{\text {finger }}=\delta_{\text {surf }}+\delta_{\text {skin }}
$$

For softer objects $d_{\text {finger }} \rightarrow \delta_{\text {surf }}$, so the object is squeezed while there is no or little skin deformation. For hard objects $d_{\text {finger }} \rightarrow \delta_{\text {skin }}$, the displacement of the finger is accompanied by a significant deformation of the skin.

\section{Warmth}

The perception of warmth is related to the change in skin temperature through the rate and direction of heat flow between the finger and the surface. This depends on the bulk material's thermal properties such as thermal diffusivity and specific heat, the density and the geometry of the contact. Heat extraction from the fingers causes a perception of coldness, or coolness, whereas the conduction of heat into the skin results in a perception of warmth. Since in most cases the temperature of the surface is at room temperature, which is lower than that of the skin of the fingerpad, which ranges from 25 to $36^{\circ} \mathrm{C}$ [11], it is the rate of heat extraction from the fingerpad skin that determines the perception of warmth. Ho and Jones [12] have shown that the cooling curves of the fingerpad can be modelled by exponential decay functions, characterized by a time constant. Different materials have different time constants, which are found to be inversely related to the logarithm of the material's thermal effusivity, which is the square root of the product of the material's thermal conductivity, specific heat and density. The thermal effusivity defines the material's ability to exchange heat with its surroundings. To be able to discriminate 
between two materials, the difference should at least vary by a factor of three in thermal effusivity, although it should be noted that the thermal effusivity does not take into account the geometry of the contact. In addition to the thermal properties of the material, the thermal contact resistance between finger and object, and the geometry of the object should be taken into account, as discussed by Bergmann Tiest [8]. The more compliant a material, the larger the contact area with the finger when the material is touched. An increase in contact area gives a larger heat transfer rate, so that softer materials feel colder. The real contact area is determined by the roughness of the material, and decreases with increasing roughness. The heat transfer rate is larger for smooth surfaces, so that smoother surfaces feel colder than rough surfaces. Ashby and Johnson [13] present the square root of the materials density $\rho$, thermal conductivity $K$ and specific heat $c_{p}$ as a measure of the coldness of the material. The quantity of heat leaving each unit of area of the fingerpad skin is proportional to this measure. The larger the quantity of heat $Q$ leaving the skin, the colder a material feels.

In a rubbing contact the contact temperature will increase proportional to the coefficient of friction following [14]

$$
\Delta T=\frac{\mu F v}{a K_{e f f}}
$$

where the effective thermal conductivity is given as

$$
K_{\text {eff }}=\frac{8}{3}\left(K_{\text {stat }}+K_{\text {mov }} \sqrt{K_{\text {mov }}+0.4 a v \rho_{\text {mov }} c_{\text {mov }}}\right)
$$

Equation (6) should be considered at various length scales. In the case of the fingerpad sliding against a smooth surface, the macro scale, the fingerpad is the stationary contact partner, whereas 'moving' applies to the countersurface. However, at the micro scale, the scale of an asperity, it is the skin that is moving against the stationary asperity summit. In this case, it is the local friction behaviour, at the asperity level, that is of interest.

\section{Roughness}

The roughness of a surface, or rather it's surface topography, is generally expressed in statistical measures which indicate the height differences of this topography measured with a profilometer, such as $R_{a}, R_{t}$ or $R_{z}$ roughess. Psychophysical roughness is related not to just one, but to a combination of these parameters, and also includes other mechanical properties of the surface, such as friction and compliance [15, 16].

When touching a rough surface, the roughness produces an uneven pressure distribution 
on the skin and vibrations when the fingerpad slides over the surface. Hollins and Bensmaïa [17] found that people spontaneously slide their finger over a surface, when asked to make judgments on its roughness. It was already reported by Taylor et al. [18] that when statically touching a rough surface, the perception of irregularities is diminished. When skin deformation changes are sufficiently rapidly to prevent adaptation and the hand moves slowly enough so that skin deformation can occur, there is no influence of sliding velocity or type of motion [15, 18, 19]. The duplex theory of tactile texture perception [17, 20] states that different mechanisms are responsible for tactile perception: a spatial code for coarse textures, with elements larger than about $100 \mu \mathrm{m}$, and a vibrotactile code for finer textures. The codes depend on the responses that arise in different types of mechanoreceptors. Hollins [20] suggests that for relatively coarse surfaces, those with elements more than about $100 \mu \mathrm{m}$ in size, information about the arrangement and density of surface features is carried from the finger to the brain primarily by SAI mechanoreceptors; the Merkel cells. For a textured surface, the SAI responses are found to be relatively sensitive to dot height and diameter [17], which are spatial parameters. For finer surfaces, vibrotactile channnels are involved, activated in part by vibrations set up when an exploring fingertip moves across a surface. Bergmann Tiest [8] argues that, since there is no direct relation between perceived roughness and sliding velocity, perceived roughness is associated with the amplitude of the vibrations, weighted with the frequency response of the Pacinian receptors.

A sample material commonly used to investigate perceived roughness is sandpaper. The perceived roughness of sandpapers is found to increase with particle diameter, or grit size, in the range 14 to $2000 \mu \mathrm{m}$, following a power law [11, 21]. Results reported by several authors show that for textured surfaces the perceived roughness increases when the distance between elements, e.g. groove width, increases: Lederman [19] found that perceived roughness increases with increasing groove width $(0.175$ to $1 \mathrm{~mm})$ and increasing pressing force $(0.27$ to $2.2 \mathrm{~N})$. Smith et al. [15] reported that perceived roughness increases with the distance between elements and height of the elements, whereas the size of the elements has a negligible effect. Kawasoe et al. [22] found that a change of height affects the perception of roughness less than a change of width does. With increasing distance between elements, the deformed volume of the fingerpad skin increases as can be shown by modelling [18].

Although correlations were found between $R_{a}$ roughness and perceived roughness [23], for random rough surfaces it appears that peak values such as $R_{z}$ and $R_{p}$ are better descriptors to relate roughness to associated feelings [2, 3]. Liu et al. [23] found a correlation of about -0.8 between surface kurtosis and perceived roughness, which indicates that the fingertip does not detect narrow large-amplitude surface features. Statistical roughness 
parameters such as the $R_{a}$ or $R_{q}$ are somehow related to asperity density and radius of curvature. Whitehouse and Archard [24] state that for many engineering surfaces the product of the density of summits $\eta$, their radius of curvature $R$ and the standard deviation of the summit height distribution $\sigma=R_{q}$ is constant. Given the fact that $\eta$ does not change very much with changing roughness, $R$ and $R_{q}$ are inversely proportional [25]. A dependence of perceived roughness on $R_{a}$ or $R_{q}$ roughness can thus be interpreted as a dependence on asperity radius of curvature, the equivalent of the particle diameter in sandpaper experiments.

Just as there is no single parameter that uniquely represents a surface's roughness, there is no single physical roughness measure that best describes what is perceived as roughness. Bergmann Tiest [16] reports that when asked what they were paying attention to while assessing roughness, subjects mentioned the presence of irregularities, ridges or bits of fluff, whether they could depress the surface, the structure and size of the irregularities, the presence of fine or coarse bumps, whether the material was hard or soft, and whether

the fingers slid easily over the surface. The definition of roughness perception has been found to be different for different people.

It may be concluded that there is not one single roughness parameter that correlates to perceived roughness, but that roughness is a multidimensional sensation that is determined by roughness amplitude, density or spacing of the asperities and friction between the surface and the fingerpad. Several studies report a positive correlation between roughness perception and friction [see e.g. 8, 15, 18, 22, 23]. However, this may be assigned to the correlation between physical roughness and friction, which will be described later in this paper.

\section{Slipperiness}

Slipperiness is the psychophysical description that is related to the friction between the finger and the surface. It has been found that slipperiness correlates well to the coefficient of friction, as shown by, for example, Liu et al. [23] who measured a correlation of 0.79 between the measured coefficient of friction and perceived slipperiness rankings using aluminium samples within a range of roughnesses.

In the sliding contact between a sphere and a smooth flat, the maximum tensile stress $\sigma_{x}$ at the surface is shown by Hamilton [9] to be proportional to the coefficient of friction following

$$
\sigma_{x}=\frac{3}{2} \frac{F}{\pi a^{2}}\left(\frac{1-2 \nu}{3}+\frac{4+\nu}{8} \pi \mu\right)
$$


whereas the magnitude of the maximum subsurface shear stress $\tau_{v M}$ is proportional to the friction as well.

$$
\tau_{v M}=\frac{3}{2} \frac{F}{\pi a^{2}}\left(\frac{(1-2 \nu)^{2}}{9}+\frac{(1-2 \nu)(2-\nu)}{12} \pi \mu+\frac{16-4 \nu+7 \nu^{2}}{192} \pi^{2} \mu^{2}\right)^{1 / 2}
$$

so that assuming a Poisson ratio $\nu_{\text {skin }}=0.49$ gives $\sigma_{x} / p_{0} \approx 1.76 \mu$ and $\tau_{v M} / p_{0} \approx 0.9 \mu$, with $p_{0}$ the maximum contact stress. Furthermore, the location of the maximum shear stress beneath the skin surface changes with the magnitude of the friction. The different mechanoreceptors in the skin detect the variation and magnitude of these stresses when the fingerpad slides against a surface, creating a certain perception of slipperiness. As with the perception of softness, Bergmann Tiest [8] argues that slipperiness is perceived through both kinaesthetic and cutaneous channels, detecting force and skin stretch respectively. These phenomena can be considered at different levels: at the level of the fingerpad sliding against a smooth surface, and at the micro level, where asperity summits are sliding against the smooth skin. The latter clarifies the importance of considering local coefficients of friction in the perception of roughness.

Bergmann Tiest [8] states that only a few studies have been carried out on the perception of slipperiness and that relatively little is known about the haptic perception of friction. It is suggested that this might be due to the fact that the friction behaviour itself cannot simply be quantified.

\section{Tactile friction}

Friction in contacts where the human fingerpad is one of the interacting surfaces is referred to as tactile friction. As opposed to grip, where contact loads up to $50 \mathrm{~N}$ may be applied, in tactile friction the touching force is low, generally in the order of several Newtons. The expressions presented in the preceeding paragraphs show that in the sliding contact between the fingerpad and a rough surface, friction plays a role in the perception of roughness, slipperiness and sometimes warmth. Statistical analysis confirms this statement, as shown by Chen et al. [2], although the correlations may be direct or indirect. They concluded that cross-correlations arise from correlations between the physical measures, such as between friction and compliance, and friction and roughness.

In engineering it is common to quantify friction and 'frictional behaviour' by the coefficient of friction, which is calculated by dividing the measured friction force by the applied normal load. Childs et al. [4] found that it is not the mean coefficient of friction that is important, but the local peak values of friction coefficient within a contact. Smith et al. [15] found a significant correlation between the friction and perceived roughness of surfaces 


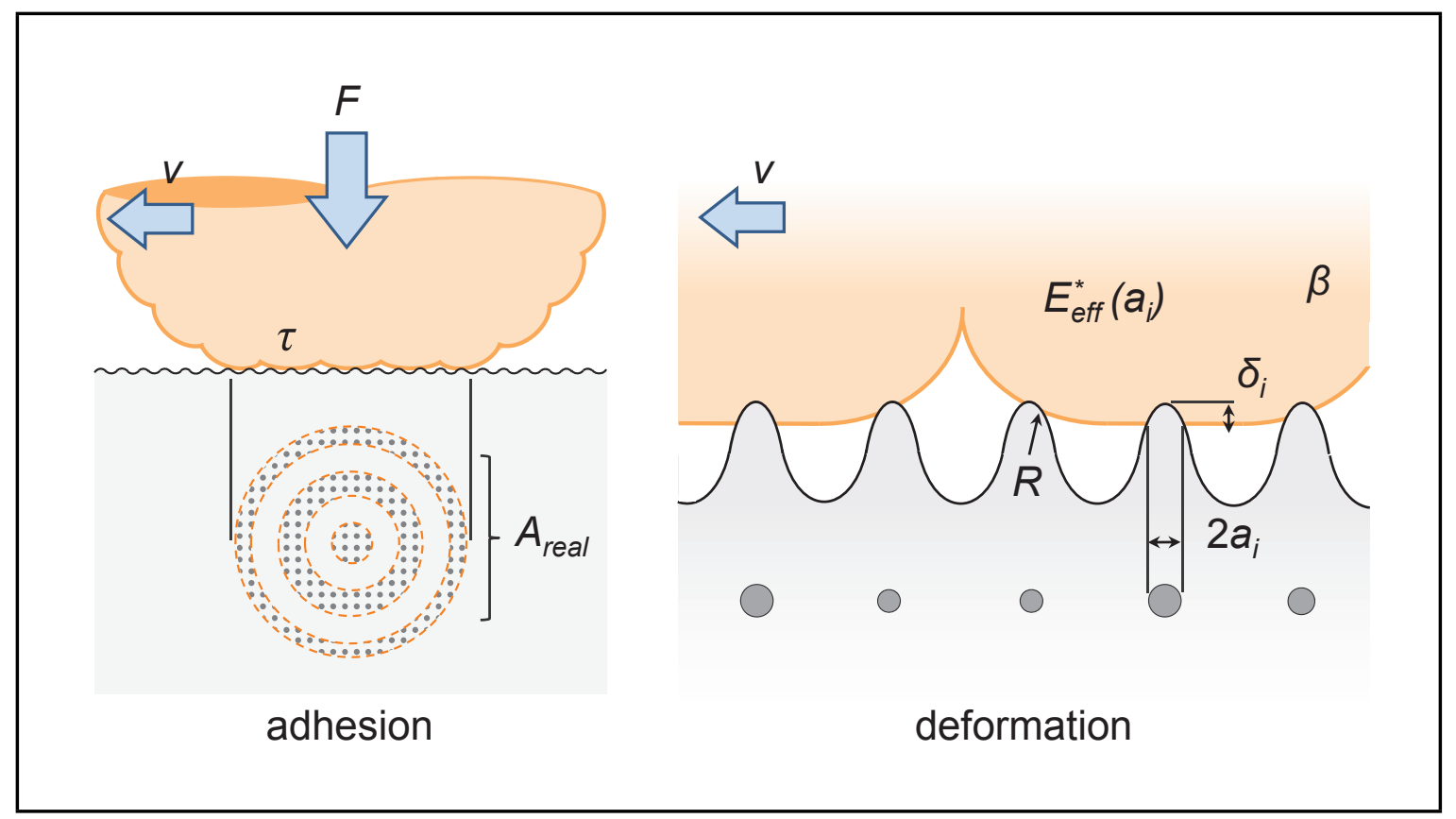

Figure 2. Schematic overview of the sliding contact between the fingerpad and a rough surface.

with arrays of raised dots. They concluded that the rate of change in the friction force seemed to be a more important parameter than the mean coefficient of friction.

Furthermore, the simplified representation of a mean coefficient of friction ignores the fact that the friction force that occurs in the contact is the result of different mechanisms. The so-called two-term model gives the friction force as the sum of an adhesion term and a deformation term $[26,27]$

$$
F_{\mu}=F_{\mu, a d h}+F_{\mu, d e f}
$$

The first term in Equation (9) can be calculated from the shear strength of the interface $\tau$ and the contact area $A$ by [28]

$$
F_{\mu, a d h}=\tau \cdot A
$$

Assuming that the interfacial shear strength $\tau$ between a probe sliding with velocity $v$ and the fingerpad rises from shear in the lipid layer which covers the skin, the interfacial shear stress can be estimated from

$$
\tau=\eta \frac{\mathrm{d} v}{\mathrm{~d} t} \approx \eta \frac{v}{t}
$$


Paper A

where $\eta$ is the dynamic viscosity and $t$ is the thickness of the lipid film. The thickness of the lipid film can be obtained from the skin surface lipid content SSL, which can be measured using a Sebumeter (Courage+Khazaka $\mathrm{GmbH}$, Germany). Typical values reported in the literature range between 1.5 and $4 \mu \mathrm{m} / \mathrm{cm}^{2}[29-31]$. From the density $\rho \approx 0.9 \mathrm{~g} / \mathrm{cm}^{3}$ and dynamic viscosity $\eta \approx 0.17 \mathrm{~Pa} \cdot \mathrm{s}$ of the lipid film [32], the layer thickness and shear stress can be calculated. Since sliding velocities in friction experiments are generally in the range 1 to $10 \mathrm{~mm} / \mathrm{s}$, an interfacial shear strength $\tau$ in the order of 10 to $100 \mathrm{kPa}$ is expected.

Adams et al. [27] argue that, for countersurfaces on which there is a thin organic film, such as skin, the interfacial shear strength is a linear function of the contact pressure $p$ following

$$
\tau=\tau_{0}+\alpha p
$$

where $\tau_{0}$ is the intrinsic interfacial shear strength, $\alpha$ is the pressure coefficient and $p$ is the mean contact pressure, which is calculated as the quotient of the applied normal load and real contact area. For wet skin sliding against a spherical glass probe they found $\tau_{0} \approx 4.8 \mathrm{kPa}$ and $\alpha \approx 0.8$, whereas for $\mathrm{PP} \tau_{0} \approx 6.1 \mathrm{kPa}$ and $\alpha \approx 2$ were found. Contact pressures in the experiments varied between 1 and $17 \mathrm{kPa}$, yielding interfacial shear strengths varying between 5 and $25 \mathrm{kPa}$ at $8 \mathrm{~mm} / \mathrm{s}$ sliding velocity. Hendriks et al. [33] adopted the same approach and estimated these parameters for dry and hydrated skin of the inner forearm. The intrinsic shear strength was found to have an order of magnitude $\tau_{0} \approx 1 \mathrm{kPa}$ and tends to increase with increasing hydration. For dry skin $\alpha \approx 0.8$ was found, increasing to $\alpha \approx 1.7$ for hydrated skin.

When an asperity indenting the skin is moved forward, work is dissipated due to the viscoelastic nature of the skin. The deformation component equals the amount of work lost by viscoelastic hysteresis per unit sliding distance, thus being proportional to a viscoelastic loss fraction $\beta$ and the indentation $\delta$ of the asperity into the skin $[26,34]$.

$$
F_{\mu, \text { def }}=\frac{3}{16} \beta \frac{\delta}{a} F
$$

For dry skin sliding against smooth surfaces the deformation term can be neglected. However, when sliding against rough surfaces, and when the skin is hydrated, deformation should be taken into account. The viscoelastic loss fraction $\beta$ can be obtained from static or dynamic indentation experiments. In a static indentation experiment $\beta$ is estimated from the area between the loading and unloading curve. Values varying between 0.24 and 0.27 are reported in the literature $[26,35,36]$. In a dynamic indentation experiment, in which a sinusoidal deformation profile is applied, $\beta$ is calculated from the viscoelastic loss 
tangent $\tan \delta$ following $\beta=\pi \tan \delta$. The viscoelastic loss tangent is defined as the ratio between the storage and loss modulus $E^{\prime \prime} / E^{\prime}$. Boyer et al. [37] found that $\beta$ increased with increasing age from $\sim 0.35$ to $\sim 0.4$ and $\sim 0.43$ for the age groups 18-30,31-50 and 51-70 years respectively. The viscoelastic loss fraction of isolated stratum corneum was measured by Yuan and Verma [38], who obtained values of 0.31 and 0.79 for dry and wet skin respectively.

It should be noted that $\beta$ should be measured at a deformation rate which is comparable to the loading-unloading rate of the sliding contact. For a sphere in sliding contact with the skin, the loading-unloading rate $v_{z}$ can be estimated from the contact dimensions and the sliding velocity following

$$
v_{z}=\frac{a}{R} v
$$

The reported data have been measured at indentation velocities between 0.06 and $2.5 \mathrm{~mm} / \mathrm{s}$, which is in accordance with the sliding velocity in tactile friction experiments, which is in general in the order of several $\mathrm{mm} / \mathrm{s}$. Figure 2 shows a schematic illustration of adhesion and deformation in a sliding contact between the fingerpad and a rough surface.

When in contact with a ridged surface an additional term, the interlocking term, can be attributed to the fingerprint ridges climbing over the surface features of the countersurface. This third term is described in further detail by Tomlinson et al. [39].

Numerous studies have reported on the friction behaviour of the human fingerpad, see for instance [25, 40-46]. A wide range of coefficients of friction is found as a function of countersurface material, surface roughness, normal load and skin condition.

\section{Contact mechanics of the fingertip}

For engineering materials, Hertz's theory $[47,48]$ provides us with expressions which describe the relation between radius of curvature $R$, normal load $F$ and the contact radius $a_{H}$ and deformation $\delta_{H}$ for a spherical contact as shown in Equations (15) and (16).

$$
\begin{aligned}
a_{\mathrm{H}} & =\left(\frac{3}{4} \frac{R F}{E^{*}}\right)^{1 / 3} \\
\delta_{\mathrm{H}} & =\left(\frac{9}{16} \frac{F^{2}}{R E^{* 2}}\right)^{1 / 3}
\end{aligned}
$$


Paper A

In which $E^{*}$ represents the reduced elastic modulus, defined as

$$
\frac{1}{E^{*}}=\frac{1-\nu_{1}^{2}}{E_{1}}+\frac{1-\nu_{2}^{2}}{E_{2}}
$$

with $E_{1}$ and $E_{2}$ the respective elastic moduli and $\nu_{1}$ and $\nu_{2}$ the Poisson ratios of the contacting materials. In the case of contact between skin and a much stiffer counterbody, $E_{\text {counterbody }} \gg E_{\text {skin }}$ and the reduced elastic modulus depends solely on the properties of the skin so that $E^{*} \approx E_{\text {skin }} /\left(1-\nu_{\text {skin }}^{2}\right)$. Since the skin is composed of different layers, and the elastic modulus of these layers decreases with depth, at different length scales different layers determine the magnitude of the apparent elasticity $E_{\text {skin }}$. To account for the multilayered and nonhomogeneous structure of the skin, Van Kuilenburg et al. [49] adopted the concept of an effective elastic modulus $E=f(a)$, providing a closedform expression describing the elasticity of the skin of the volar forearm as a function of length scale. In the case of a spherical contact, the length scale of the contact equals the contact radius $a$. Figure 3 shows that the effective elastic modulus of the volar forearm varies considerably with length scale. At different scales, the evolution of the effective modulus with length scale can be described by power laws. Although the anatomy of the fingerpad is different from the volar forearm and the skin differs in layer thickness and surface topography, the same trend is expected for the skin of the fingerpad. At the micro scale, i.e. the scale of surface roughness, the effective elastic modulus is determined by the top layer of the skin, the stratum corneum. With increasing length scales, the lower, and more compliant, skin layers will determine the elastic behaviour, so that a decrease of the effective elastic modulus is expected. Finally, the much stiffer bone of the distal phalanx causes the elasticity to increase. The length scale effect determines the contact behaviour of the human skin, such as the growth of the fingerpad area with normal load.

\section{Contact area}

Different measuring methods have been used to measure the relation between the apparent contact area $A_{0}$ and applied load $F$. The main difference is the principle which is used for measuring the contact area for different values of the applied normal load. A relatively simple method is to use an inkpad and to press the inked finger on a piece of smooth paper at different normal loads [4, 50-53]. The normal load can be monitored using a load cell or even a kitchen scale. The size of the fingerprints can be measured using image analysis software.

To observe the contact area and applied load simultaneously, some researchers use a dedicated set-up to measure the contact area optically using microscopy [54-57] or optical 


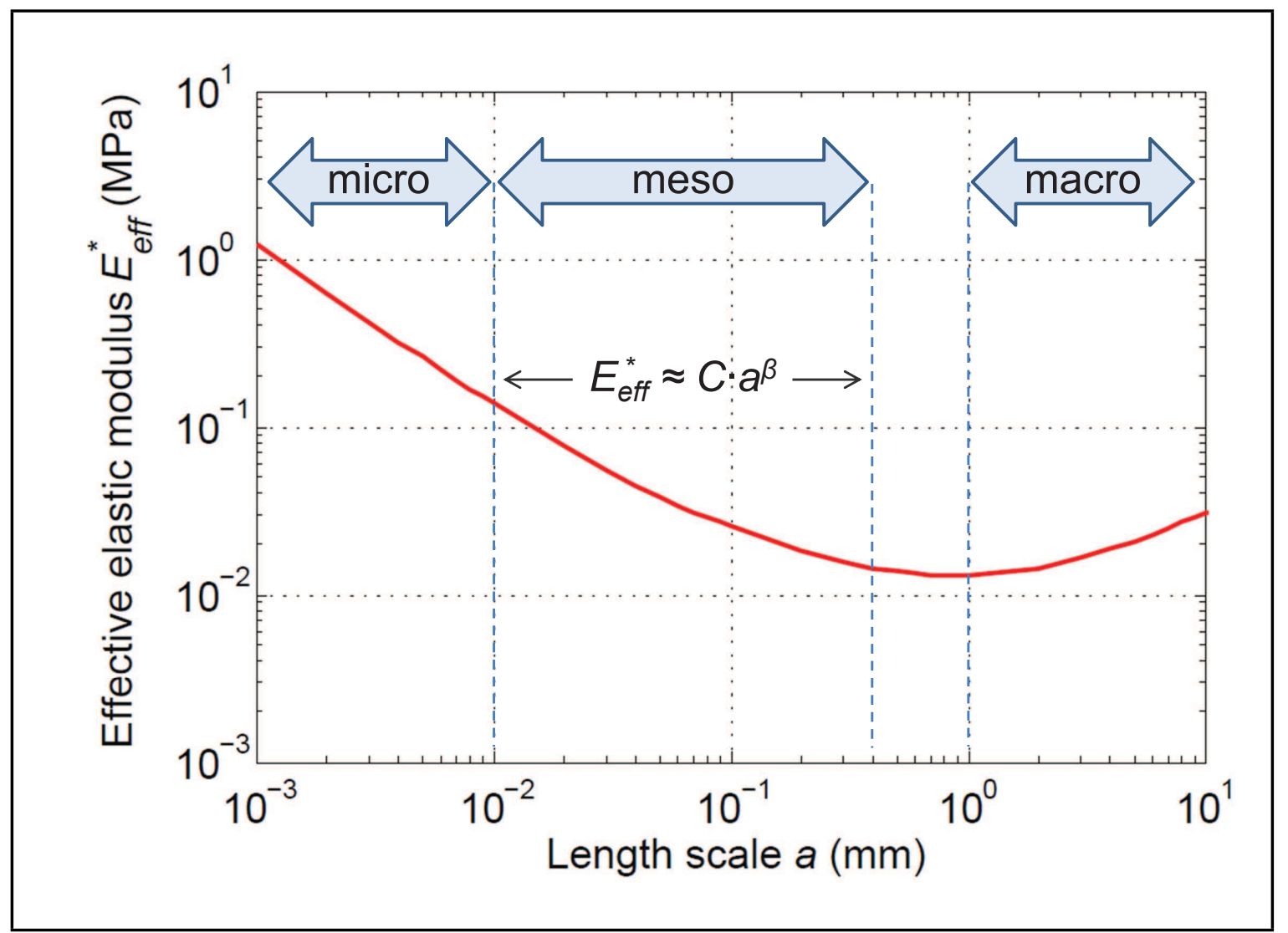

Figure 3. Effective elastic modulus of the skin of the volar forearm as a function of contact length scale. Figure adapted from [49]. 
Paper A

coherence tomography [58]. An indirect method is the use of a pressure sensitive film $[59,60]$.

Analysis of data collected from the literature $[4,18,40,50,52-57,59,60]$ shows that as a function of normal load the measured apparent contact area follows a power law

$$
A_{0} \propto F^{n}
$$

with exponents ranging between 0.15 and 1.33 . Obviously the relation between the contact area of the fingertip and normal load deviates from the behaviour predicted by equation (15), which gives an exponent $n=0.66$.

The exponent of the power law fitted through the measurement results depends on the range of applied normal loads. At higher loads, the effective elastic modulus increases with normal load. From Equations (15) and (18) it can be seen that when the effective elastic modulus of the fingertip increases with normal load, an exponent $n<0.66$ is expected. At low loads, the relation between normal load and apparent contact area follows a power law with an exponent larger than 0.66. The results obtained by Maeno et al. [54] illustrate those two regimes. To approximately $0.1 \mathrm{~N}$ a steep increase of the apparent contact area with normal load is observed, characterized by $n \approx 1.33$, whereas from 0.1 to $1 \mathrm{~N}$, a weaker decrease gives $n \approx 0.35$. Warman and Ennos [52] showed that, at loads between 0 and $1.6 \mathrm{~N}$, the exponent $n$ depends on the finger and the orientation of the finger. The middle and ring finger yield higher exponents (0.75 and 0.85) than the index finger (0.54). For the index finger the fingerpad yields a higher exponent (0.54) than the tip of the finger (0.43). For the fingerpad and fingertip respectively, exponents 0.55 and 0.36 were found by Tomimoto et al. [53].

Figure 4 shows an overview of data collected from the literature for the apparent contact area measured as a function of normal load. At $1 \mathrm{~N}$ normal load the contact area varies between approximately 80 and $200 \mathrm{~mm}^{2}$. The contact area of the index finger decreases with increasing orientation angle, at $1 \mathrm{~N}$ normal load being approximately $155 \mathrm{~mm}^{2}$ for the fingerpad, $40 \mathrm{~mm}^{2}$ at intermediate orientation and $20 \mathrm{~mm}^{2}$ for the tip as found by Warmann and Ennos [52]. Kinoshita et al. [50] found that the thumb contact area was $\sim 1.5$ times the area of the index finger regardless of the applied normal force for four subjects.

As well as the apparent contact area, the real contact area follows a power law with normal load. Soneda and Nakano measured the real area of contact of the fingertip against smooth glass, finding exponents between 0.58 and 0.81 [56]. At small loads the contact ratio increases with normal load from 0.27 to 0.43 [61], whereas at higher loads the contact ratio was found to be independent of normal load and a contact ratio varying between 


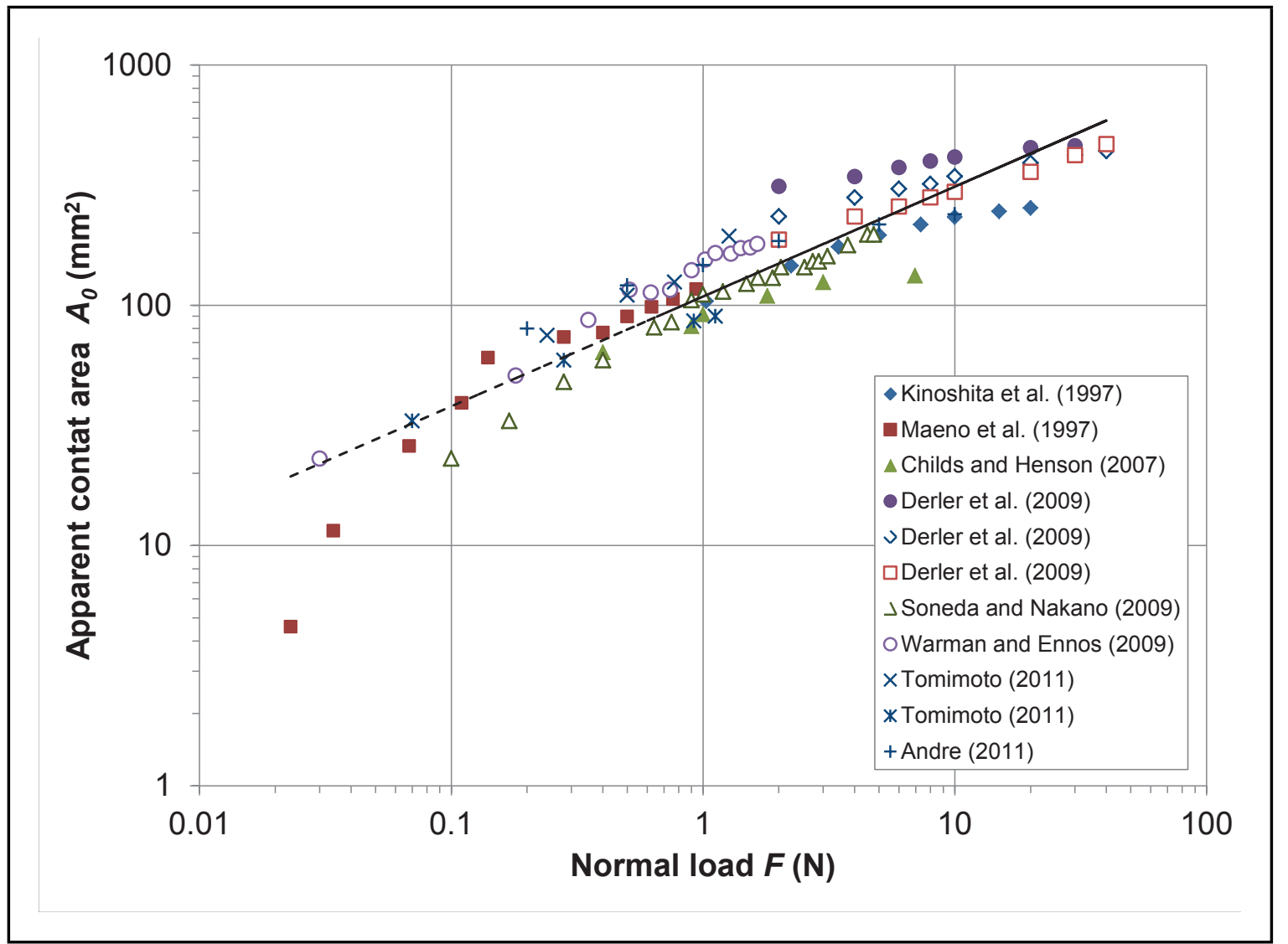

Figure 4. Apparent contact area of the fingerpad as a function of normal load. Data taken from Kinoshita et al. [50], Maeno et al. [54], Childs and Henson [4], Derler et al. [59], Soneda and Nakano [56], Warmann and Ennos [52], Tomimoto [53] and André et al. $[57]$. 
Paper A

0.43-0.59 was found [51]. Assuming that the real area of contact increases by fresh ridges of the fingerprint pattern coming into contact rather than ridges increasing in width [40], the real contact area can be calculated from the density and the width of the fingerprint ridges. The topography of the skin of the fingerpad, the fingerprint ridges, can be obtained from a three-dimensional surface topography measurement e.g. using a silicone replica of the fingerprint skin.

\section{Hydration}

The fingerprint ridges contain sweat glands, which form single rows of pores of diameter 5-20 $\mathrm{mm}$ [41]. The number of pores per unit of area varies considerably between subjects. Liu et al. [58] measured between 310 and 1090 sweat ducts per $\mathrm{cm}^{2}$ measured for seven subjects. The sweat ducts supply the skin of the fingerpad with a small amount of sweat to enhance grip by increasing adhesion. Besides, hydration of the top layer of the skin decreases its stiffness [38, 49, 62], thus increasing the real contact area.

The hydration level of the skin can be measured using a Corneometer CM 825 (Courage + Khazaka GmbH, Germany). The Corneometer measures the changing capacitance of a precision capacitor in arbitrary units AU, which represent the change in the dielectric constant due to hydration of the skin surface, up to a depth of 10-20 $\mu \mathrm{m}$. The Corneometer is an established equipment for measuring hydration in skin tribology studies, [see e.g. 6365]. Typical Corneometer readings are 20 AU for dry skin, 55 AU for normal skin and $85 \mathrm{AU}$ for moist skin [66]. Another device to measure the amount of skin moisture is the Moistsense [66].

Even when in the same environment or doing the same activity, the hydration level varies between subjects [66], although no correlation has been found between the number of sweat ducts and the hydration level [58].

The hydration level increases significantly when the subject is active, and with the duration of the activity [66]. Especially, when holding an object, such as a pen during writing, the moisture content at the interface is influenced by the occlusion time. Pasumarty et al. [67] suggest that the occlusion time is a critical factor that determines friction behaviour of skin.

\section{Fingertip friction}

In an early study on fingertip friction carried out using nearly 400 schoolchildren, Spurr [40] measured coefficients of friction ranging between 0.1 and 1.2. The average coefficient of friction obtained from measurements carried out on different days ranged between 0.42 
and 0.54. Similar results were obtained on 30 adult subjects, where an average coefficient of friction of 0.48 was measured within a range $0.2-0.9$. More recently Liu et al. [58] measured the friction of the fingerpad sliding against steel for seven subjects. The coefficient of friction varied between 0.38 and 2.19 and an average of 1.03 was found with a standard deviation $\sigma=0.64$. These panel studies clearly show that the friction measured between the fingertip and a certain countersurface is subject to various sources of variation.

\section{Measuring}

In tribology it is common to measure friction and wear using a sphere-on-flat configuration, such as a pin-on-disk tribometer, under well-controlled conditions. The initial Hertzian contact stress is determined by the sample geometry and applied load, mostly applied using a dead weight. Friction and sometimes wear depth are measured instantaneously. A well-accepted method for measuring tactile friction is the principle of a stationary sample that is attached to a load measuring device over which the subjects slide their fingerpads. In several studies researchers use a load cell [5, 15, 25, 45, 46, 59, 68-71], although sometimes a custom-made force plate is used $[22,23,41,43,44,53,58,72,73]$. As opposed to the subject applying a normal load and imposing a sliding motion, in a machine controlled experiment the fixated finger is in contact with a sliding probe [67].

An important aspect in tribological research is the characterization of sample surfaces before and after testing, such as determination of the elastic modulus, hardness, surface topography and surface chemistry. In skin tribology some dedicated equipment is used to evaluate the condition of the skin surface. The hydration level of the skin, which interacts with the mechanical and surface chemical properties of the skin, is measured using an apparatus such as the Corneometer, whereas the lipid content can be measured using a Sebumeter.

\section{Variation}

The frictional behaviour of two surfaces that are in sliding contact depends on the surfaces, any lubricant and the environmental conditions as well as the operational conditions [74]. Any changes in these contact conditions will inevitably lead to a noticeable variation in

friction results. The variation which is observed when analyzing skin friction data, whether those data are collected from the literature or obtained from an experimental program, can be attributed to several sources. Considering operational conditions such as the normal load, the sliding velocity and the hydration level of the skin as the experimental parameters still leaves several variables which may influence the results. An overview of studies which 
Paper A

Table 1. Sources of variation.

\begin{tabular}{|c|c|c|c|c|}
\hline Study & Variable & Range & Countersurface & Coefficient of friction \\
\hline Spurr [40] & gender & & metal cooking foil & girls $>$ boys \\
\hline Sasada [42] & gender & & steel & $\begin{array}{l}\text { female }>\text { male }(<20 \mathrm{yrs}) \\
\text { female }<\text { male }(>20 \mathrm{yrs})\end{array}$ \\
\hline Ramalho et al. [68] & gender & & glass & female $<$ male \\
\hline Savescu et al. [70] & gender & & $\begin{array}{l}\text { sandpaper, silk, polyester } \\
\text { cotton, rayon }\end{array}$ & $\begin{array}{l}\text { female }>\text { male } \\
\text { female }<\text { male }\end{array}$ \\
\hline Derler et al. [59] & gender & & glass & female $<$ male \\
\hline Sasada [42] & age & $0 \ldots 80$ years & steel & decreases \\
\hline Han et al. [72] & orientation angle & $30^{\circ}, 45^{\circ}, 60^{\circ}$ & PMMA & decreases \\
\hline Childs et al. [4] & orientation angle & $0^{\circ}, 45^{\circ}$ & textured polymer & decreases \\
\hline Tomimoto [53] & orientation angle & $0^{\circ}, 80^{\circ}$ & PMMA & decreases \\
\hline Gee et al. [43] & sliding direction & left-right & miscellaneous & constant \\
\hline Skedung et al. [45] & sliding direction & back-forth & paper & constant \\
\hline
\end{tabular}

report on the influences of subject gender and age, orientation angle of the finger and type of motion is given in Table 1 .

Most authors observed a lower friction for adult females than for adult males. Below a certain age the friction observed for female subjects, or rather girls, is higher than for boys $[40,42]$. In a study on the friction between the fingerpad and different textiles Savescu et al. [70] measured a lower friction for females than for males, although against some materials this effect was reversed. When sliding against relatively hard surfaces such as steel or glass, the lower friction observed for females can be explained from the contact behaviour of the fingertip. Generally women have a smaller fingertip than men, so the contacting area between the finger and the countersurface will be smaller, hence the lower friction. Sasada [42] further reports the influence of age: for both males and females a decrease of the coefficient of friction was observed when the age increases from 0 to 80 years.

After receiving instructions, in most studies the subject controls the position and motion of the fingertip. The orientation of the finger is often referred to as 'pad', 'tip' or 'intermediate'. It has been shown that the orientation angle has a significant influence on the coefficient of friction, the friction decreasing with increasing orientation angle [4, 53, 72]. As discussed earlier in this paper, Warman and Ennos [52] showed that the contact area, and its relation to applied load, depends strongly on the orientation angle. No significant differences have been reported whether sliding from left to right [43], nor between back and forth [45].

Before carrying out a tribological experiment it is common to clean the sample material in an ultrasonic bath using solvents such as ethanol or acetone. In most studies involving the fingerpad as one of the contacting partners, before the experiments the hands are washed with soap and air dried to achieve a condition which can be referred to as 'untreated normal skin' $[23,25,41,44-46,58,60,66,70-72,75]$. To achieve a condition that more closely resembles practical applications Derler et al. [69] applied no cleaning or treatment of the skin at all. 
The thin lipid layer which may be present on the skin alters the friction behaviour. It could increase the friction through its adhesive properties, but this same lipid layer might also act as a lubricating layer on the interface, which against a smooth counter surface forms a friction reducing layer. To remove the lipid layer on the skin, it can be cleaned with ethanol $[42,53,59,60,73]$, propanol [43] or another surfactant [67]. Sasada [42] found that after cleaning the skin with ethanol, the measured friction is very low. However, after 10 minutes a gradual generation of sweat was observed at the finger surface and the friction increased. Spurr [40] observed oily contamination which remained after sliding a clean finger over glass and found that only a little contamination can reduce the measured friction. Sliding the finger over a previously used part of the surfaces resulted in significantly lower friction. This same effect was observed by Skedung et al. [45], who measured a decrease in coefficient of friction against paper with the number of repetitions. XPS measurements showed that lipid material was transferred to the paper. The effect was stronger for smoother papers. A layer of transferred lipid material was observed by Dinç et al. [41] as well.

\section{Hydration}

Consistent results have been reported over the years showing that the coefficient of friction increases considerably with increasing hydration level of the skin [25, 41, 58, 66, 67, 69].

Masen [25] found an influence of the countersurface roughness on the effect of hydration: whereas for intermediate roughness $\left(R_{q}\right.$ between $0.005 \mu \mathrm{m}$ and $\left.1 \mu \mathrm{m}\right)$ a two- to threefold increase in the coefficient of friction was measured, for surfaces that are relatively smooth or relatively rough the increase in the coefficient of friction for hydrated skin is only moderate.

The hydration level of the skin increases as a result of ambient relative humidity, occlusion or application of water onto the skin. Pasumarty et al. [67] found that the coefficient

of friction increases with increasing contact time, or occlusion time, until it reaches a steady-state value.

Tomlinson et al. $[66,75]$ showed that friction increases with increasing hydration level of the skin, whereas above a certain level the presence of moisture at the skin surface reduces friction again.

\section{Normal load}

In engineering applications the real contact area of the tribological contacts grows proportional to the normal load so that Amontons' law of friction applies, which states that the friction force is proportional to the normal load: the coefficient of friction has a constant value. Some authors report a linear dependence of the friction force on normal load. In 
Paper A

one of the earliest studies on fingertip friction, Spurr [40] found that the friction force was approximately proportional to the load. Against a smooth glass surface Ramalho et al. [68] measured that the friction force is proportional to the normal load within a large load range from 0 to $70 \mathrm{~N}$. Also, at lower loads against textured polymers [4], different types of textiles [70] and paper [45], a linear relation was observed. In a comparative study Tomlinson et al. [44] found that the friction force and normal load showed a linear relation between 0 and $40 \mathrm{~N}$ normal load for different types of materials; metals, plastics as well as elastomers.

However, for materials such as elastomers and skin the real contact area is a function of normal load. The friction force, and the coefficient of friction, are expected to depend on the applied normal load. Several authors report that the coefficient of friction decreases with normal load following a power law with exponents between -0.68 and -0.24 [42, 46, $60,70,72,73]$

Masen [25] found a more complex friction behaviour as the measured coefficient of friction varied strongly with normal load. At low loads $(1 \mathrm{~N})$ the coefficient of friction is relatively high $(\mu \approx 1)$. At a load in the order of $2.5 \mathrm{~N}$ it reaches a minimum $(\mu \approx 0.3)$ after which it increases with increasing load $(\mu \approx 0.4$ at $5 \mathrm{~N}$ load). Seo et al. [71] report that the coefficient of friction decreased when the normal load increased from 1 to $10 \mathrm{~N}$. A further increase to $20 \mathrm{~N}$ normal load had no significant effect. An increase at higher normal loads was found by Liu et al. [58] who reported a linear increase of the coefficient of friction when the normal load increases from 7.5 to $25 \mathrm{~N}$.

The behaviour might depend on the countersurface material. Dinç et al. [41] report that the coefficient of friction against polymers, such as PMMA, PA and PC, decreases with friction when the normal load increases from 1 to $20 \mathrm{~N}$. The same trend was observed independent of roughness and ambient humidity. However, when sliding against PTFE the coefficient of friction remained constant.

Tomimoto [53] showed that for the fingerpad $\left(\varphi \approx 0^{\circ}\right)$ the coefficient of friction decreases with increasing normal load, whilst for the fingertip $\left(\varphi \approx 90^{\circ}\right)$ it remains approximately constant.

Derler et al. [59] report friction as a function of normal load in the range 0 to $50 \mathrm{~N}$ against smooth and rough glass. They found that the coefficient of friction decreases with increasing normal load when the fingertip is sliding against smooth glass, for both dry and wet skin. The decrease with increasing load was larger for females than for males, and against the wet glass the decrease was larger than against the smooth glass. When sliding against rough glass the coefficient of friction in the dry state is almost independent of normal load.

Figure 5 shows data for the coefficient of friction of the fingerpad obtained from the 


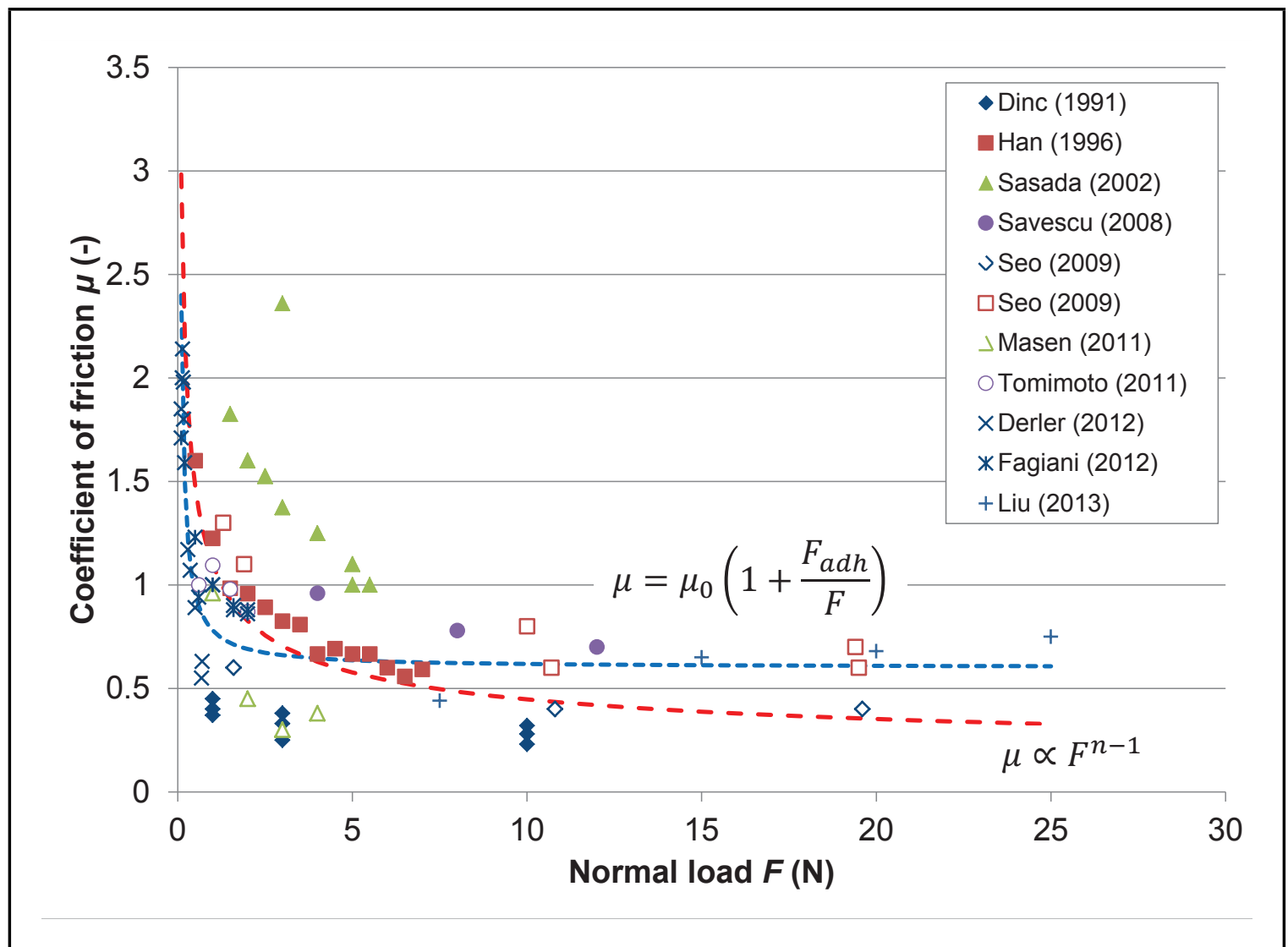

Figure 5. Coefficient of friction of the index finger as a function of normal load. Data taken from Dinç et al. [41], Han et al. [72], Sasada [42], Savescu et al. [70], Seo et al. [71], Masen [25], Tomimoto [53], Derler et al. [59], Fagiani et al. [73], Liu et al.[58] 
Paper A

literature. Although the data is obtained from different studies, carried out in different laboratories, using different subjects and against different surfaces, the data show a consistent trend of a coefficient of friction decreasing with increasing normal load. The figure points out that the strongest decrease occurs at relatively low normal loads. This can be explained from the evolution of the contact area with increasing normal load following a power law with exponent $n$. Assuming that the friction force is proportional to the contact area gives for the relation between the coefficient of friction and normal load

$$
\mu \propto F^{n-1}
$$

A fit through all data shown in Fig. 4 gives $n=0.54$, so that $n-1=-0.46$. A line representing this relation is drawn in Fig. 5.

Calculating the measured coefficient of friction by dividing the measured friction force by the applied normal load does not take into account the effect of adhesion on the contact load. What is actually measured is the friction force that results from the real normal load $F_{\text {real }}$, which is the applied load plus the adhesion force between the two surfaces

$$
\begin{aligned}
F_{\mu} & =\mu_{\text {real }} \cdot F_{\text {real }} \\
& =\mu_{\text {real }} \cdot\left(F+F_{\text {adh }}\right)
\end{aligned}
$$

When the measured coefficient of friction $\mu$ is calculated by using Eq. (1), what is actually calculated is

$$
\mu=\mu_{\text {real }} \cdot\left(1+\frac{F_{a d h}}{F}\right)
$$

Equation (21) shows that the effect of adhesion becomes important when the normal load is of the same order of magnitude as the adhesion force, i.e. at relatively low normal loads. A line representing this relation is drawn in Fig. 5.

\section{Velocity}

Only a few studies report on the influence of sliding velocity on the measured friction. A decrease of the coefficient of friction with increasing sliding velocity $(6-60 \mathrm{~mm} / \mathrm{s})$ was observed by Dinç et al. [41] for different plastics at normal and high humidity. An exception was PTFE for which the friction remained constant. Fagiani et al. [73] found that the friction between the fingerpad and aluminium was only slightly affected by the sliding velocity $(10-30 \mathrm{~mm} / \mathrm{s})$, the coefficient of friction decreasing with increasing velocity. When sliding against different types of textile they found that the coefficient of friction tends 
to decrease when the sliding velocity increases within the range $10-50 \mathrm{~mm} / \mathrm{s}$. Recently, Derler et al. [60] found that when sliding against wet smooth glass the coefficient of friction decreases with sliding velocity $(0-1500 \mathrm{~mm} / \mathrm{s})$ following a power law with exponent -0.37 . Although in general the coefficient of friction of the fingerpad tends to decrease with increasing sliding velocity, Pasumarty et al. [67] observed different trends depending on the type of material and condition of the skin. Within the velocity range between 3 and $24 \mathrm{~mm} / \mathrm{s}$ the coefficient of friction against glass was almost independent of sliding velocity, whereas against PP a slight decrease was observed and friction against steel was found to increase with velocity. Against hydrated skin the coefficient of friction for these materials was found to be independent of sliding velocity.

\section{Material}

The data collected from the literature covers a wide range of materials, but differences in test conditions make it difficult to compare between studies. Only a few studies provide a comparison of the friction behaviour of different materials.

Sasada [42] measured the friction as a function of $R_{a}$ roughness for PMMA and a ceramic. For a roughness between 4 and $12 \mu \mathrm{m} R_{a}$ the coefficient of friction against PMMA is lower than against the ceramic. Results of an experimental program using different types of materials are reported by Liu et al. [23]. Comparing the results obtained using samples having a comparable roughness in terms of $R_{a}$ shows that the coefficient of friction against aluminium is larger than against wood and ABS. The friction measured against PP is higher than measured against aluminium. These results correspond to observations by Tomlinson et al. [75] who found higher coefficients of friction for PP than for metal. The friction they observed against different soft elastomers is even higher. Pasumarty et al. [67] measured a coefficient of friction between 0.2 and 0.24 between the fingerpad and smooth glass $\left(R_{a}=0.01 \mu \mathrm{m}\right)$ which is considerably lower than the coefficient of friction they measured against smooth steel $\left(R_{a}=0.05 \mu \mathrm{m}\right)$, between 0.5 and 1 .

\section{Roughness}

In most studies the surface topography of the sample is characterized by the centre line average roughness, in other words the $R_{a}$ roughness; a measure commonly used in mechanical engineering. This parameter basically is the arithmetic mean of the surface heights relative to the mean line and in general gives an acceptable indication of the roughness of a test surface.

Dinç et al. [41] found that the coefficient of friction between the fingerpad and PMMA decreased when the $R_{a}$ roughness increased from 0.033 to 1.55 and $6.15 \mu \mathrm{m}$. This effect 
Paper A

was found to be independent of load $(1-20 \mathrm{~N})$ and sliding velocity $(6-60 \mathrm{~mm} / \mathrm{s})$ and was observed for dry as well as hydrated skin. The results correspond to the observations by Sasada [42] who found a decrease in friction when the $R_{a}$ roughness of the PMMA countersurface increased from 0.2 to $10.5 \mathrm{\mu m}$. In the same study a decrease was observed when sliding against porous ceramics $\left(R_{a}=4.1-22 \mu \mathrm{m}\right)$. The same trends were observed against metals such as aluminium, when the roughness increased from 0.3 to $13 \mu \mathrm{m} R_{a}$ [23] and steel, when the roughness increased from 0.003 to $1.44 \mu \mathrm{m} R_{a}$ [25]. When sliding against different types of paper Skedung et al. [45] found that rougher papers tend to result in lower coefficients of friction than smoother papers $\left(R_{a}=1-4 \mu \mathrm{m}\right)$.

A completely different behaviour is reported for very rough surfaces by Tomlinson et al. [44], who report for different metals an increase of the coefficient of friction with roughness, from $0.46 \mu \mathrm{m} R_{a}$ onwards. For roughness values exceeding approximately $26 \mu \mathrm{m}$ $R_{a}$, the friction did not increase any further. An increase of the friction of dry skin was also measured against glass when the $R_{a}$ roughness increased from 0.01 to $4.2 \mu \mathrm{m}$ by Pasumarty et al. [67]. On the contrary, when sliding against hydrated skin they observed a considerable decrease of the friction with roughness.

Amplitude parameters such as the $R_{a}$ roughness are, in fact, statistical parameters describing the height distribution of the measured profile. Those parameters do not contain any information about size, shape or density of the asperities. It is beyond doubt that these properties also influence the surface topography and the frictional behaviour. The surface topography is the result of the finishing method that is used. Gee et al. [43] compared samples with roughness amplitudes, in terms of $R_{a}$ roughness, of $0.8,3.2$ and $25 \mu \mathrm{m}$, finished using different techniques. It was found that the coefficient of friction decreased with $R_{a}$ roughness when the surfaces were spark eroded and increased when the surfaces were grit blasted or ground. For planed surfaces the friction showed a minimum at intermediate $R_{a}$ roughness, being higher for both smoother and rougher surfaces. Smith et al. [15] investigated the friction behaviour of the fingerpad against macro-textures built up from $1.8 \mathrm{~mm}$ amplitude truncated cones. They found that the coefficient of friction increased when the spatial period between the cones increased from 1.5 to $8.5 \mathrm{~mm}$. The friction behaviour between the fingerpad and rectangular ridged metal surfaces was investigated by Tomlinson et al. [76]. They found that the coefficient of friction increased with increasing ridge height $(0.5-2.5 \mathrm{~mm})$ and increasing spacing $(2-12 \mathrm{~mm})$. The coefficient of friction decreased with increasing ridge width $(1-4 \mathrm{~mm})$, whereas from 4-8 $\mathrm{mm}$ no change was observed. 


\section{Summarizing conclusion}

When touching a product's surface, mechanical and thermal receptors in the skin are stimulated and give rise to sensorial judgments of softness, warmth, roughness and slipperiness. The phenomena occurring in the contact between the fingerpad and the surface, which give rise to these perceptions, can be understood by considering deformations, subsurface stresses and vibrations in the skin. Contact mechanics theory shows that the friction against the skin influences the magnitudes, locations and gradients of subsurface stresses and strains and temperatures, thus influencing the perception of roughness, slipperiness and sometimes warmth.

For product engineers who aim to control and optimize the sensorial properties of a product surface interacting with the skin, it is essential to understand this frictional behaviour. However, the friction of skin is yet poorly understood. In the literature, a wide range of coefficients of friction as a function of countersurface material, surface roughness, normal load and skin condition is reported. The variation that is observed within or between studies can be assigned to gender, age and orientation of the finger. Together with the skin condition, these parameters should be taken into account when comparing different studies. Despite these sources of variation, which are intrinsic to tactile exploration, analyzing data collected from the literature shows some consistent trends:

- The coefficient of friction increases considerably with increasing hydration level of the skin, mainly due to softening of the top layer of the skin.

- Measurements show that the contact area of the fingerpad changes with normal load following a power law. Since the friction force is proportional to the contact area, the coefficient of friction changes with normal load. Although there is a large variation in geometry and properties of the fingerpad between different people, measurements of the mechanical behaviour of the fingerpad show a consistent trend. The relation between contact area and normal load can easily be evaluated using a load measuring device and an inkpad in combination with image analysis software.

- The coefficient of friction of the fingerpad decreases with normal load to a constant value. The decrease, which is most clear at relatively low loads, below $5 \mathrm{~N}$, can be attributed to the effect of normal adhesion and to the contact behaviour of the fingerpad. At low normal loads the contribution of the adhesion force between the skin and the countersurface becomes apparent, leading to an increase in friction with decreasing normal load.

- The coefficient of friction changes with surface roughness. Data collected from the literature show that for low values of $R_{a}$ roughness, friction decreases with increasing 
Paper A

roughness, caused by a decrease in the real contact area. When the $R_{a}$ roughness increases further, the contribution of deformation causes an increase in the friction, after which the coefficient of friction remains constant. Although it is arguable whether the $R_{a}$ roughness is a suitable parameter to describe surface roughness, the trends found in the literature are consistent. However, some influence of the finishing method is reported. It should be noted, that when investigating roughness perception, alternative roughness parameters should be included as well.

Analysis of the tactile qualities described from a tribological point of view shows that in the sliding contact between the fingerpad and a rough surface, friction plays a role in the perception of warmth, roughness and slipperiness. In a rubbing contact the contact temperature increases proportional to the coefficient of friction. The relation between perceived roughness and friction is less obvious and may be assigned to the correlation which exists between physical roughness and friction. The perception of slipperiness has been show to correlate to the frictional properties of the surface. Slipperiness can be related to the magnitude and location of (sub)surface stresses and strains in the skin, which are proportional to the coefficient of friction.

Even though the coefficient of friction of the fingerpad shows some consistent trends, examining the friction behaviour at a more detailed level might further explain the contribution of friction to tactile perception. The measuring signal contains relevant information, such as local peaks in friction and friction versus time gradients, and should be analyzed thoroughly as opposed to taking the average coefficient of friction of the steady-state part of the signal. Furthermore, future work should involve the study of local friction behaviour at the scale of the surface roughness.

\section{Funding}

This research was carried out under project number M63.2.10409 in the framework of the Research Program of the Materials innovation institute M2i (www.m2i.nl).

\section{References}

1. E Karana, $\mathrm{P}$ Hekkert, and $\mathrm{P}$ Kandachar. Meanings of materials through sensorial properties and manufacturing processes. Materials \& Design, 30(7):2778-2784, 2009.

2. X Chen, CJ Barnes, THC Childs, B Henson, and F Shao. Materials' tactile testing and characterisation for consumer products' affective packaging design. Materials \& Design, 30(10):4299-4310, 2009. 
3. CJ Barnes, THC Childs, B Henson, and CH Southee. Surface finish and touch - a case study in a new human factors tribology. Wear, 257(7-8):740-750, 2004.

4. THC Childs and B Henson. Human tactile perception of screen-printed surfaces: Selfreport and contact mechanics experiments. Proceedings of the Institution of Mechanical Engineers, Part J: Journal of Engineering Tribology, 221(3):427-441, 2007.

5. MA Darden and CJ Schwartz. Investigation of skin tribology and its effects on the tactile attributes of polymer fabrics. Wear, 267(5-8):1289-1294, 2009.

6. L Skedung, K Danerlöv, U Olofsson, CM Johannesson, M Aikala, J Kettle, M Arvidsson, B Berglund, and MW Rutland. Tactile perception: Finger friction, surface roughness and perceived coarseness. Tribology International, 44(5):505-512, 2011.

7. S Schreiner, M Rechberger, and J Bertling. Haptic perception of friction - correlating friction measurements of skin against polymer surfaces with subjective evaluations of the surfaces' grip. Tribology International, 63:21-28, 2013.

8. WM Bergmann Tiest. Tactual perception of material properties. Vision Research, 50(24):2775-2782, 2010.

9. GM Hamilton. Explicit equations for the stresses beneath a sliding spherical contact. Proceedings of the Institution of Mechanical Engineers, Part C: Journal of Mechanical Engineering Science, 197(1):53-59, 1983.

10. S Derler and L-C Gerhardt. Tribology of skin: Review and analysis of experimental results for the friction coefficient of human skin. Tribology Letters, 45:1-27, 2011.

11. RT Verrillo, SJ Bolanowski, CM Checkosky, and FP McGlone. Effects of hydration on tactile sensation. Somatosensory \&3 Motor Research, 15(2):93-108, 1998.

12. H-N Ho and LA Jones. Contribution of thermal cues to material discrimination and localization. Perception $\& 3$ psychophysics, 68(1):118-128, 2006.

13. M Ashby and K Johnson. Materials and design - The art and science of material selection in product design. Elsevier, Oxford, 2006.

14. E van der Heide and DJ Schipper. On the frictional heating in single summit contacts: Towards failure at asperity level in lubricated systems. Journal of Tribology, 126(2):275-280, 2004. 
15. AM Smith, CE Chapman, M Deslandes, J-S Langlais, and M-P Thibodeau. Role of friction and tangential force variation in the subjective scaling of tactile roughness. Experimental Brain Research, 144(2):211-223, 2002.

16. WM Bergmann Tiest and AML Kappers. Haptic and visual perception of roughness. Acta Psychologica, 124(2):177-189, 2007.

17. M Hollins and SJ Bensmaïa. The coding of roughness. Canadian Journal of Experimental Psychology, 61(3):184-195, 2007.

18. MM Taylor and SJ Lederman. Tactile roughness of grooved surfaces: A model and the effect of friction. Perception \&3 Psychophysics, 17(1):23-36, 1975.

19. SJ Lederman. The perception of surface roughness by active and passive touch. Bulletin of the Psychonomic Society, 18(5):253-255, 1981.

20. M Hollins, SJ Bensmaïa, and S Washburn. Vibrotactile adaptation impairs discrimination of fine, but not coarse, textures. Somatosensory 83 Motor Research, 18(4):253-262, 2001.

21. SS Stevens and JR Harris. The scaling of subjective roughness and smoothness. Journal of Experimental Psychology, 64(5):489-494, 1962.

22. T Kawasoe, M Kakizawa, and H Shimizu. Tribology in the hair surface and tactile perception. Tribology Online, 3(2):127-130, 2008.

23. X Liu, Z Yue, Z Cai, DG Chetwynd, and ST Smith. Quantifying touch-feel perception: tribological aspects. Measurement Science and Technology, 19(8):084007, 2008.

24. DJ Whitehouse and JF Archard. The properties of random surfaces of significance in their contact. Proceedings of the Royal Society of London. Series A, Mathematical and Physical Sciences, 316(1524):97-121, 1970.

25. MA Masen. A systems based experimental approach to tactile friction. Journal of the Mechanical Behavior of Biomedical Materials, 4:1620-1626, 2011.

26. SA Johnson, DM Gorman, MJ Adams, and BJ Briscoe. The friction and lubrication of human stratum corneum. In D Dowson and CM Taylor, editors, Thin Films in Tribology, Proceedings of the 19th Leeds-Lyon Symposium on Tribology held at the Institute of Tribology, University of Leeds, volume 25, pages 663-672. Elsevier, 1993.

27. MJ Adams, BJ Briscoe, and SA Johnson. Friction and lubrication of human skin. Tribology Letters, 26(3):239-253, 2007. 
28. FP Bowden and D Tabor. The friction and lubrication of solids. Cambridge University Press, Oxford, 1950.

29. AB Cua, KP Wilhelm, and HI Maibach. Skin surface lipid and skin friction: relation to age, sex and anatomical region. Skin Pharmacology, 8(5):246-251, 1995.

30. A Mavon, H Zahouani, D Redoules, P Agache, Y Gall, and Ph Humbert. Sebum and stratum corneum lipids increase human skin surface free energy as determined from contact angle measurements: A study on two anatomical sites. Colloids and Surfaces B: Biointerfaces, 8(3):147-155, 1997.

31. L-C Gerhardt, A Lenz, ND Spencer, T. Münzer, and S Derler. Skin-textile friction and skin elasticity in young and aged persons. Skin Research and Technology, 15(3):288298, 2009.

32. L-C Gerhardt, A Schiller, B Müller, ND Spencer, and S Derler. Fabrication, characterisation and tribological investigation of artificial skin surface lipid films. Tribology Letters, 34(2):81-93, 2009.

33. CP Hendriks and SE Franklin. Influence of surface roughness, material and climate conditions on the friction of human skin. Tribology Letters, 37(2):361-373, 2009.

34. JA Greenwood and D Tabor. The friction of hard sliders on lubricated rubber: The importance of deformation losses. Proceedings of the Physical Society, 71(6):989-1001, 1958.

35. C Flynn, A Taberner, and P Nielsen. Measurement of the force-displacement response of in vivo human skin under a rich set of deformations. Medical Engineering 83 Physics, 33(5):610-619, 2011.

36. J Jachowicz, R McMullen, and D Prettypaul. Indentometric analysis of in vivo skin and comparison with artificial skin models. Skin Research and Technology, 13(3):299-309, 2007.

37. G Boyer, L Laquièze, A Le Bot, S Laquièze, and H Zahouani. Dynamic indentation on human skin in vivo: ageing effects. Skin Research and Technology, 15(1):55-67, 2009.

38. Y Yuan and R Verma. Measuring microelastic properties of stratum corneum. Colloids and Surfaces B: Biointerfaces, 48(1):6-12, 2006.

39. SE Tomlinson, MJ Carré, R Lewis, and SE Franklin. Human finger contact with small, triangular ridged surfaces. Wear, 271(9-10):2346-2353, 2011. 
Paper A

40. RT Spurr. Fingertip friction. Wear, 39(1):167-171, 1976.

41. OS Dinç, CM Ettles, SJ Calabrese, and HA Scarton. Some parameters affecting tactile friction. Journal of Tribology, 113(3):512-517, 1991.

42. T Sasada. The friction of human skin. In Proceedings of the 21st Biotribology Symposium, JSME, Fukuoka, 2000, pages 46-52, 2000.

43. MG Gee, P Tomlins, A Calver, RH Darling, and M Rides. A new friction measurement system for the frictional component of touch. Wear, 259(7-12):1437-1442, 2005.

44. SE Tomlinson, R Lewis, and MJ Carré. The effect of normal force and roughness on friction in human finger contact. Wear, 267(5-8):1311-1318, 2009.

45. L Skedung, K Danerlöv, U Olofsson, M Aikala, K Niemi, J Kettle, and MW Rutland. Finger friction measurements on coated and uncoated printing papers. Tribology Letters, 37(2):389-399, 2010.

46. S Derler, J Süess, A Rao, and G-M Rotaru. Influence of variations in the pressure distribution on the friction of the finger pad. Tribology International, 63:14-20, 2013.

47. H Hertz. Über die Berührung fester elastischer Körper. Journal für die reine und angewandte Mathematik, 92:156-171, 1882.

48. KL Johnson. Contact mechanics. Cambridge University Press, Cambridge, 1999.

49. J Van Kuilenburg, MA Masen, and E Van der Heide. Contact modelling of human skin: What value to use for the modulus of elasticity? Proceedings of the Institution of Mechanical Engineers, Part J: Journal of Engineering Tribology, 227(4):347-359, 2013.

50. H Kinoshita, L Bäckström, JR Flanagan, and RS Johansson. Tangential torque effects on the control of grip forces when holding objects with a precision grip. Journal of Neurophysiology, 78(3):1619-1630, 1997.

51. SE Tomlinson. Understanding the friction between human fingers and contacting surfaces. PhD thesis, The University of Sheffield, 2009.

52. PH Warman and AR Ennos. Fingerprints are unlikely to increase the friction of primate fingerpads. Journal of Experimental Biology, 212(13):2016-2022, 2009.

53. M Tomimoto. The frictional pattern of tactile sensations in anthropomorphic fingertip. Tribology International, 44(11):1340-1347, 2011. 
54. T Maeno, K Kobayashi, and N Yamazaki. Relationship between the structure of human finger tissue and the location of tactile receptors. JSME International Journal Series C, 41:94-100, 1998 .

55. N Nakazawa, R Ikeura, and H Inooka. Characteristics of human fingertips in the shearing direction. Biological Cybernetics, 82(3):207-214, 2000.

56. T Soneda and K Nakano. Investigation of the vibration detectability of human fingers supported by the observation of contact zones. In Proceedings of the 2nd International Conference on Advanced Tribology, Singapore, 2008, paper no. iCAT288, 2008.

57. T André, V Lévesque, V Hayward, P Lefèvre, and J-L Thonnard. Effect of skin hydration on the dynamics of fingertip gripping contact. Journal of The Royal Society Interface, 8(64):1574-1583, 2011.

58. X Liu, Z Lu, R Lewis, MJ Carré, and SJ Matcher. Feasibility of using optical coherence tomography to study the influence of skin structure on finger friction. Tribology International, 63:34-44, 2013.

59. S Derler, L-C Gerhardt, A Lenz, E Bertaux, and M Hadad. Friction of human skin against smooth and rough glass as a function of the contact pressure. Tribology International, 42(11-12):1565-1574, 2009.

60. S Derler and G-M Rotaru. Stick-slip phenomena in the friction of human skin. Wear, 301(1-2):324-329, 2013.

61. T Soneda and K Nakano. Investigation of vibrotactile sensation of human fingerpads by observation of contact zones. Tribology International, 43(1-2):210-217, 2010.

62. C Pailler-Mattéi, S Pavan, R Vargiolu, F Pirot, F Falson, and H Zahouani. Contribution of stratum corneum in determining bio-tribological properties of the human skin. Wear, 263(7-12):1038-1043, 2007.

63. AB Cua, KP Wilhelm, and HI Maibach. Frictional properties of human skin: relation to age, sex and anatomical region, stratum corneum hydration and transepidermal water loss. British Journal of Dermatology, 123(4):473-479, 1990.

64. L-C Gerhardt, V Strässle, A Lenz, ND Spencer, and S Derler. Influence of epidermal hydration on the friction of human skin against textiles. Journal of The Royal Society Interface, 5(28):1317-1328, 2008. 
Paper A

65. M Kwiatkowska, SE Franklin, CP Hendriks, and K Kwiatkowski. Friction and deformation behaviour of human skin. Wear, 267(5-8):1264-1273, 2009.

66. SE Tomlinson, R Lewis, X Liu, C Texier, and MJ Carré. Understanding the friction mechanisms between the human finger and flat contacting surfaces in moist conditions. Tribology Letters, 41(1):283-294, 2011.

67. Friction of the human finger pad: Influence of moisture, occlusion and velocity. 44(2).

68. A Ramalho, CL Silva, AACC Pais, and JJS Sousa. In vivo friction study of human palmoplantar skin against glass. In Proceedings of the Nordtrib 2006-12th Nordic Tribology Symposium, Denmark, 2006.

69. S Derler, U Schrade, and L-C Gerhardt. Tribology of human skin and mechanical skin equivalents in contact with textiles. Wear, 263(7-12):1112-1116, 2007.

70. AV Savescu, ML Latash, and VM Zatsiorsky. A technique to determine friction at the fingertips. Journal of Applied Biomechanics, 24(1):43-50, 2008.

71. NJ Seo and TJ Armstrong. Friction coefficients in a longitudinal direction between the finger pad and selected materials for different normal forces and curvatures. Ergonomics, 52(5):609-616, 2009.

72. H-Y Han, A Shimada, and S Kawamura. Analysis of friction on human fingers and design of artificial fingers. In Proceedings of the 1996 IEEE International Conference on Robotics and Automation, volume 4, pages 3061-3066. IEEE, 1996.

73. R Fagiani, F Massi, E Chatelet, JP Costes, and Y Berthier. Contact of a finger on rigid surfaces and textiles: Friction coefficient and induced vibrations. Tribology Letters, 48(2):145-158, 2012.

74. H Czichos. Tribology: A systems approach to the science and technology of friction, lubrication and wear. Elsevier, Amsterdam, 1978.

75. S Tomlinson, R Lewis, S Ball, A Yoxall, and M Carré. Understanding the effect of finger-ball friction on the handling performance of rugby balls. Sports Engineering, 11(3):109-118, 2009.

76. SE Tomlinson, R Lewis, MJ Carré, and SE Franklin. Human finger friction in contacts with ridged surfaces. Wear, 301(1-2):330-337, 2013. 


\section{Appendix}




Paper A

$\sigma \quad$ stress $(\mathrm{Pa})$

$\tau \quad$ shear stress $(\mathrm{Pa})$ 



\section{Paper B}

Contact modelling of human skin: What value to use for the modulus of elasticity?

J. van Kuilenburg, M.A. Masen and E. van der Heide

Proceedings of the IMechE Part J: Journal of Engineering Tribology,

Vol.227, No.4, 2013, pp. 347-359

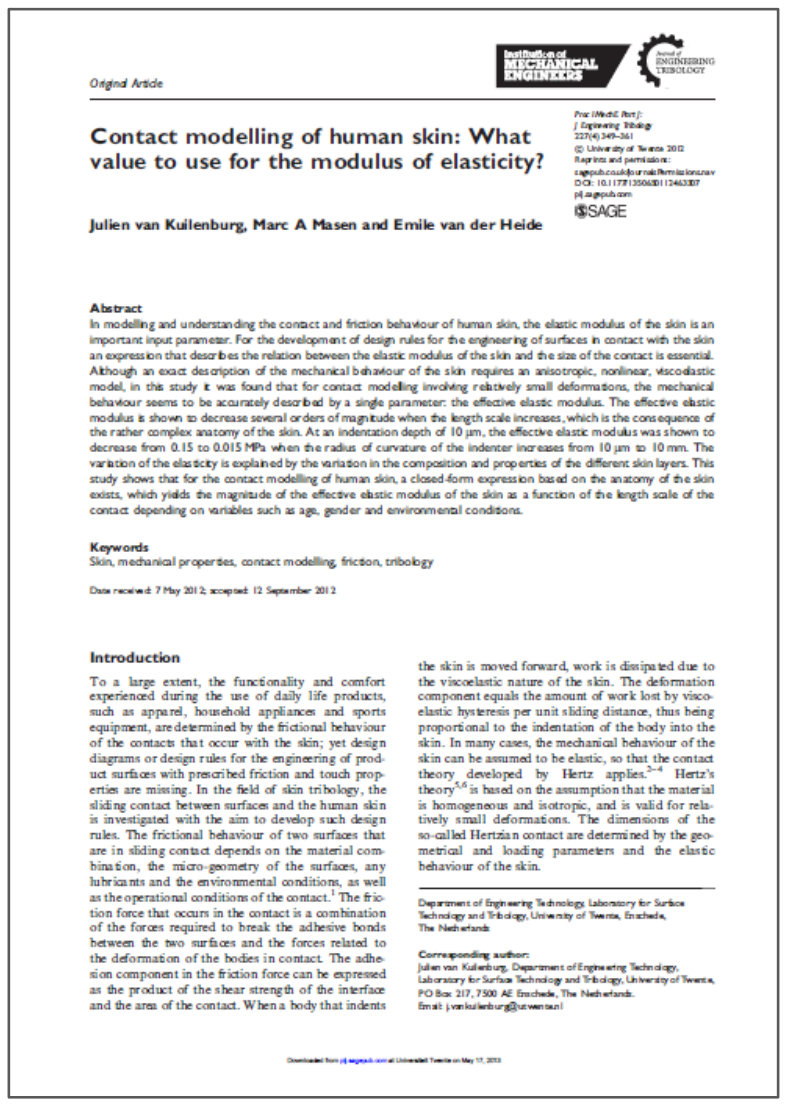





\title{
Contact modelling of human skin: What value to use for the modulus of elasticity?
}

\author{
Julien van Kuilenburg, Marc A. Masen and Emile van der Heide
}

\begin{abstract}
In modelling and understanding the contact and friction behaviour of human skin, the elastic modulus of the skin is an important input parameter. For the development of design rules for the engineering of surfaces in contact with the skin, it is essential to have an expression that describes the relation between the elastic modulus of the skin and the size of the contact.

Although an exact description of the mechanical behaviour of the skin requires an anisotropic, nonlinear, viscoelastic model, in this study it was found that for contact modelling involving relatively small deformations, the mechanical behaviour seems to be accurately described by a single parameter: the effective elastic modulus. The effective elastic modulus is shown to decrease several orders of magnitude when the length scale increases, which is the consequence of the rather complex anatomy of the skin. At an indentation depth of $10 \mu \mathrm{m}$, the effective elastic modulus was shown to decrease from 0.15 to 0.015 $\mathrm{MPa}$ when the radius of curvature of the indenter increases from $10 \mu \mathrm{m}$ to $10 \mathrm{~mm}$. The variation of the elasticity is explained by the variation in the composition and properties of the different skin layers.

This study shows that for the contact modelling of human skin, a closed-form expression based on the anatomy of the skin exists, which yields the magnitude of the effective elastic modulus of the skin as a function of the length scale of the contact, depending on variables such as age, gender and environmental conditions.
\end{abstract}

\section{Keywords}

Skin, mechanical properties, contact modelling, friction, tribology 


\section{Introduction}

To a large extent, the functionality and comfort experienced during the use of everyday products, such as apparel, household appliances and sports equipment, are determined by the frictional behaviour of the contacts that occur with the skin; yet design diagrams or design rules for the engineering of product surfaces with prescribed friction and touch properties are missing. In the field of skin tribology, the sliding contact between surfaces and the human skin is investigated with the aim of developing such design rules. The frictional behaviour of two surfaces that are in sliding contact depends on the material combination, the micro-geometry of the surfaces, any lubricants and the environmental conditions, as well as the operational conditions of the contact [1]. The friction force that occurs in the contact is a combination of the forces required to break the adhesive bonds between the two surfaces and the forces related to the deformation of the bodies in contact. The adhesion component in the friction force can be expressed as the product of the shear strength of the interface and the area of the contact. When a body that indents the skin is moved forward, work is dissipated due to the viscoelastic nature of the skin. The deformation component equals the amount of work lost by viscoelastic hysteresis per unit sliding distance, thus being proportional to the indentation of the body into the skin. In many cases, the mechanical behaviour of the skin can be assumed to be elastic, so that the contact theory developed by Hertz applies [2-4]. Hertz's theory [5, 6] is based on the assumption that the material is homogeneous and isotropic, and is valid for relatively small deformations. The dimensions of the so-called Hertzian contact are determined by the geometrical and loading parameters and the elastic behaviour of the skin.

In modelling and understanding the contact and friction behaviour of human skin, the elastic modulus of the skin $E$ is thus an essential input parameter [7, 8]. As a material, skin behaves in a complex manner; it has a layered structure with highly changing properties through the layers, its behaviour is viscoelastic, anisotropic and there may be an influence of underlying tissue and bones. The properties of the skin vary with anatomical location and with subject, age, gender, level of care and hydration. The different layers of the skin have different thicknesses and mechanical properties. The outermost surface of the skin, the stratum corneum, is a 20-40 $\mu \mathrm{m}$ thick layer, which is composed of hornified

cells giving this layer its high stiffness, whereas the thickness of the extremely viscous and soft hypodermis ranges from 1 to $50 \mathrm{~mm}$, depending on anatomical site and dietary habits $[9,10]$. Common methods for quantifying the skin mechanical behaviour are extension, torsion, suction and indentation. When investigating the friction behaviour of human skin, a commonly used experimental set-up is the spherical probe sliding over the skin. At the microlevel, the contact between a rough surface and the skin can be imagined as a collection 
of spherical tipped asperities, each asperity indenting the skin [11]. This is why the indentation principle is the most appropriate technique for measuring the mechanical properties which determine the contact and friction behaviour of human skin. Elastic moduli reported throughout the literature have been measured with a wide range of indenter geometries, making a straight translation to the particular geometry of interest difficult. Even though numerous results from experiments using spherical indenters can be found in the literature, the variation of used tip radii and normal loads does not allow an easy comparison of these values. The inner, also volar, forearm is a site commonly used for investigating tribological and mechanical properties of the skin: not only is it representative for nonglabrous skin, but it also has a relatively low hair fibre density and its surface is relatively smooth. Figure 1 shows data for the measured elastic modulus of in vivo skin of the inner forearm obtained from the literature. Reported data for the measured elastic modulus of isolated stratum corneum as a function of indentation depth are shown in Figure 2.

Figures 1 and 2 point out that different elastic moduli should be taken into account whether modelling macrocontacts, such as skin in contact with product parts, or microcontacts, such as skin, i.e. stratum corneum, against surface features. For the purpose of contact modelling, it is of practical use to present the skin elastic properties as a function of the size of the contact. For an axisymmetric indenter this characteristic length scale can be the contact radius $a$. The appropriate value of $E$ to be used should be determined at the correct length scale and under representative conditions. A simple expression describing the relation between the elastic modulus of the skin and the size of the contact from microlevel to macrolevel is currently not available. For the development of design rules for the engineering of surfaces in contact with the skin such an expression $E=f(a)$ is essential.

\section{Theory}

The contact of an individual asperity is generally modelled as a spherical contact. To a first approximation, Johnson et al. [12] and Adams et al. [19] give the total friction force of a spherical indenter sliding against the human skin by the sum of two non-interacting terms.

$$
F_{\mu}=F_{\mu, a d h}+F_{\mu, d e f}
$$




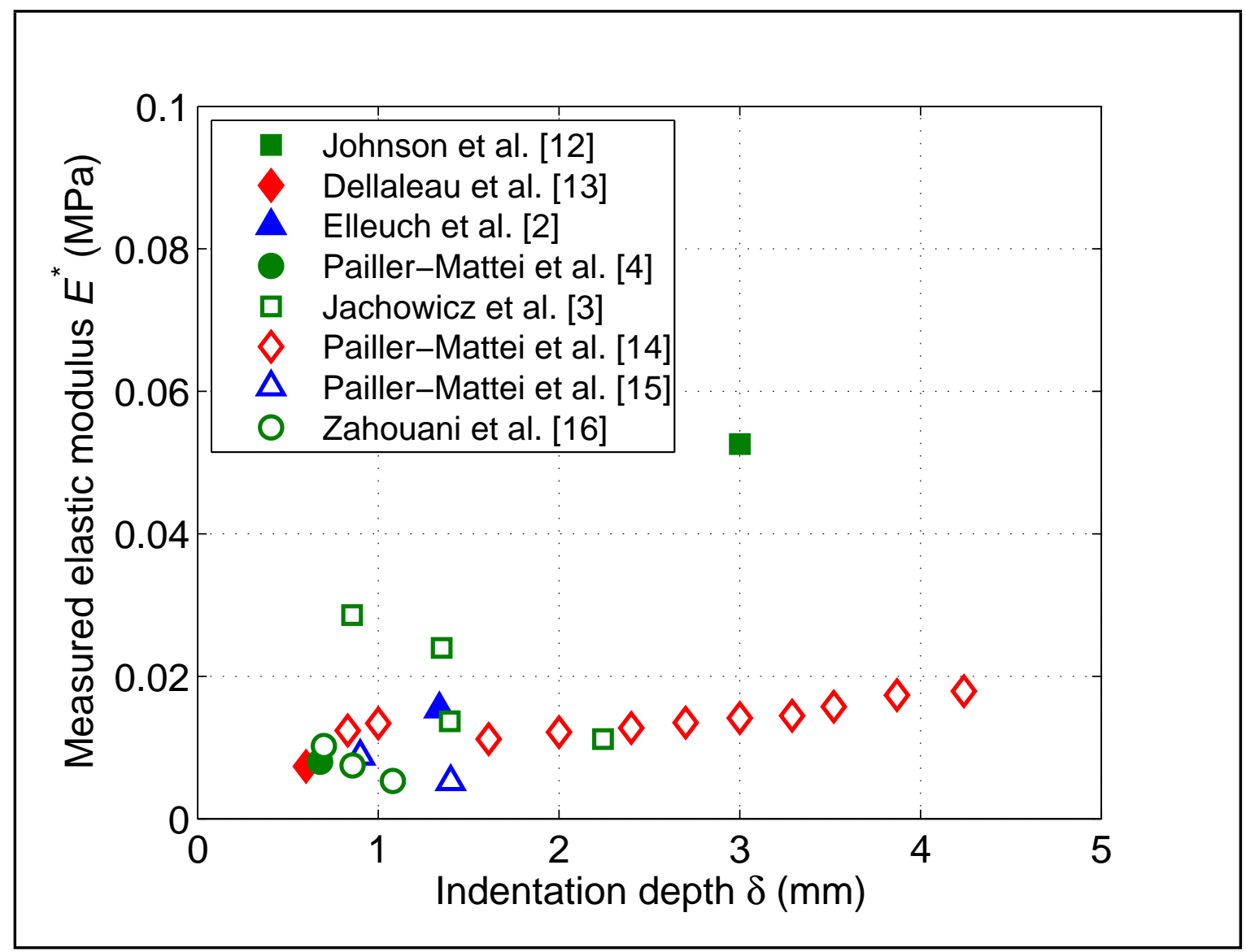

Figure 1. Measured elastic modulus of in vivo skin of the human volar forearm as a function of indentation depth. Data taken from [2-4, 12-16]. 


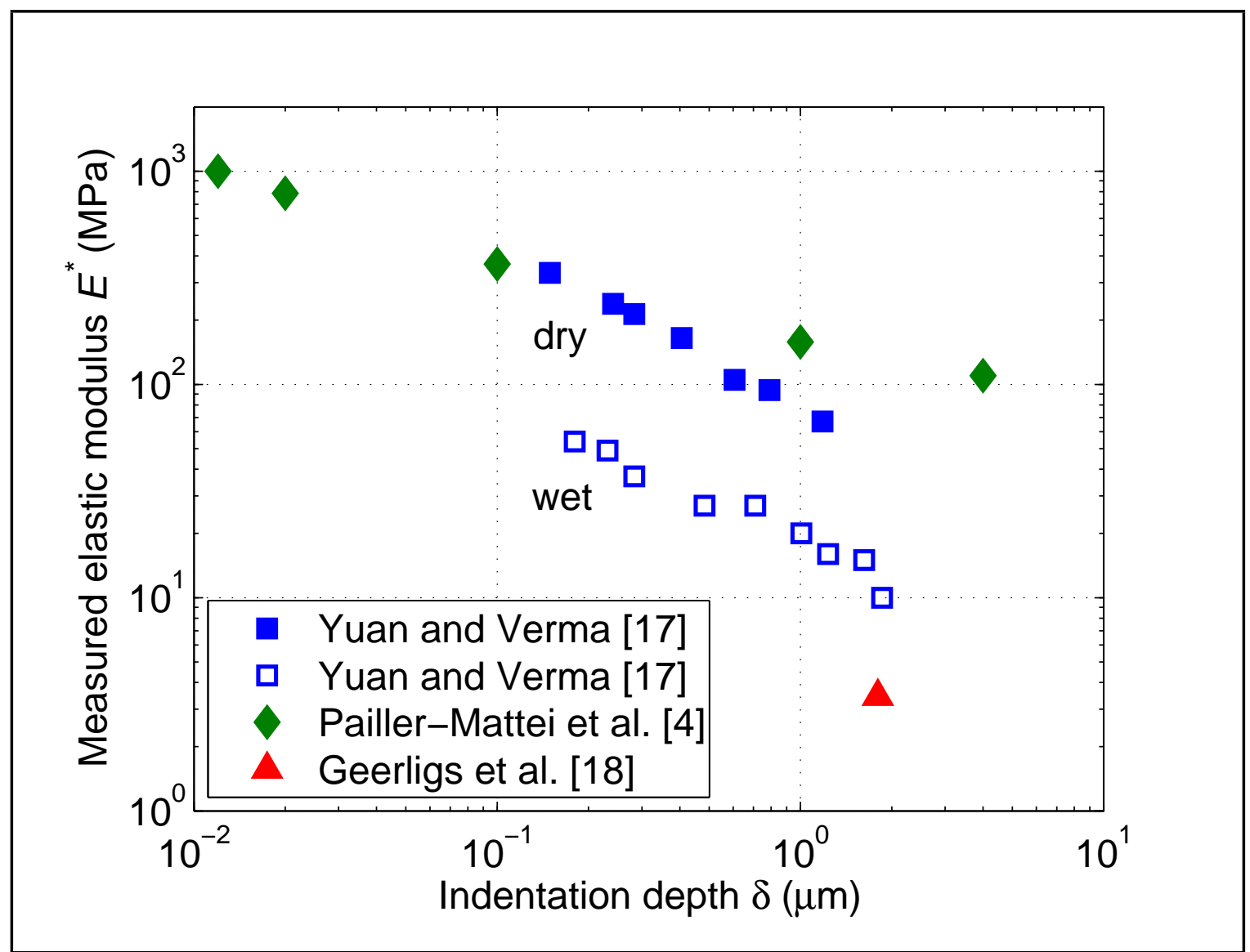

Figure 2. Measured elastic modulus of isolated stratum corneum as a function of indentation depth. Data taken from $[4,17,18]$. 
The first term in equation (1) can be calculated from the shear strength of the interface $\tau$ and the contact radius $a$ by

$$
F_{\mu, a d h}=\tau \pi a^{2}
$$

When a spherical probe indenting the skin is moved forward, work is dissipated due to the viscoelastic nature of the skin. The deformation component equals the amount of work lost by viscoelastic hysteresis per unit sliding distance, thus being proportional to a viscoelastic loss fraction $\beta$ and the indentation $\delta$ of the probe into the skin.

$$
F_{\mu, d e f}=\frac{3}{16} \beta \frac{\delta}{a} F
$$

By regarding the skin as an elastic half-space loaded over a small circular region of its plane surface, the contact radius $a$ and indentation depth $\delta$ can be calculated from the normal load $F$ and radius of curvature of the body $R$ using Hertz's theory as shown in Equations (4) and (5) respectively.

$$
\begin{aligned}
& a=\sqrt[3]{\frac{3}{4} \frac{R F}{E^{*}}} \\
& \delta=\sqrt[3]{\frac{9}{16} \frac{F^{2}}{R E^{* 2}}}
\end{aligned}
$$

In which $E^{*}$ represents the reduced elastic modulus, defined as

$$
\frac{1}{E^{*}}=\frac{1-\nu_{1}^{2}}{E_{1}}+\frac{1-\nu_{2}^{2}}{E_{2}}
$$

with $E_{1}$ and $E_{2}$ the respective elastic moduli and $\nu_{1}$ and $\nu_{2}$ the Poisson ratios of the contacting materials. In the case of contact between skin and a stiff counterbody, $E_{\text {counterbody }} \gg$ $E_{\text {skin }}$ and the reduced elastic modulus depends solely on the properties of the skin so that $E^{*} \approx E_{\text {skin }} /\left(1-\nu_{\text {skin }}^{2}\right)$. However, the skin is a layered material, being composed of three layers; the epidermis, the underlying connective tissue called the dermis and the hypodermis, or subcutis, consisting of fatty connective tissue, that connects the dermis to the underlying muscles or bones. Therefore it is questionable whether a simple elastic parameter such as the elastic modulus can be used to accurately describe the behaviour of a complex material such as the human skin. 


\section{Mechanical properties of the skin}

For measuring the mechanical properties as they apply to a tribological contact in which the skin is one of the contacting surfaces, the indentation technique yields the proper results as discussed in the introduction. In an indentation experiment, an instrument continuously measures force and displacement applied to a rigid indenter. Depending on the positioning accuracy of the instrument used, mechanical properties can be determined down to the submicron level, so for skin at the level of the stratum corneum. The indentation technique can be applied in vitro on isolated skin samples or in vivo, then always measuring the combined response of the different skin layers. For any axisymmetric indenter, the reduced elastic modulus $E^{*}$ of the indented material can be obtained from the initial unloading slope of the load-displacement curve, which plots the normal load $F$ versus indentation $\delta$ as proposed by Oliver and Pharr [20].

$$
E^{*}=\frac{\sqrt{\pi}}{2 \sqrt{A}} \frac{\partial F}{\partial \delta}
$$

The projected area of the elastic contact $A$ can be determined from the indenter geometry and the indentation depth. For a spherical indenter, the contact radius $a$ is a function of indentation depth $\delta$ and tip radius $R$ given by Hertz as

$$
a=\sqrt{\delta R}
$$

For a conical indenter, the contact radius is related to the indentation depth through the half angle $\alpha$ of the tip so that

$$
a=\frac{2}{\pi} \frac{\delta}{\tan \alpha}
$$

For a Berkovich indenter, the projected area $A$ is obtained from the area function $A=$ $24.5 \delta^{2}$, which relates the area to the indentation depth [20]. For the purpose of scaling the results obtained with a Berkovich indenter to those obtained using an axisymmetric indenter, an equivalent contact radius is obtained from $A=\pi a^{2}$ so that $a \approx 2.8 \delta$.

A less common method to obtain the reduced elastic modulus is to fit the loaddisplacement data to the Hertz Equation [3, 12]. Some studies calculate the elastic modulus by numerical analysis of the indentation curve $[2,13]$. 


\section{Stratum corneum and epidermis}

At the length scale of surface roughness, i.e. smaller than $10 \mu \mathrm{m}$, the elastic properties of the skin are determined by the top layer of the skin. Several studies report the mechanical properties of isolated stratum corneum as a function of indentation depth determined from indentation experiments, shown in Figure 2. Yuan and Verma [17] obtained elastic moduli for dry and wet isolated porcine stratum corneum. Load-displacement curves were measured using a Berkovich tip. For dry stratum corneum, the reduced elastic modulus decreased from order $300 \mathrm{MPa}$ at $0.2 \mu \mathrm{m}$ indentation to about $75 \mathrm{MPa}$ at $1 \mu \mathrm{m}$. For wet stratum corneum, a considerably lower elasticity was measured decreasing from about 50 $\mathrm{MPa}$ to $10 \mathrm{MPa}$ at $0.2 \mu \mathrm{m}$ and $2 \mu \mathrm{m}$ indentation, respectively.

Pailler-Mattei et al. [4] compared mechanical and tribological properties of isolated stratum corneum with in vivo measurements. Isolated human stratum corneum with a thickness around 10-20 $\mu \mathrm{m}$ was attached to a glass plate. Indentation experiments were carried out using a spherical diamond tip with $7.8 \mu \mathrm{m}$ radius. By extrapolation, the elastic modulus of the skin outer surface was obtained, being around 1 GPa. The elastic modulus then decreased with increasing indentation depth to around $100 \mathrm{MPa}$ at $4 \mu \mathrm{m}$.

Geerligs et al. [18] carried out indentation tests on isolated human stratum corneum and isolated human epidermis using a spherical sapphire tip with $500 \mu \mathrm{m}$ radius. For the stratum corneum, a reduced elastic modulus of $3.44 \pm 0.8 \mathrm{MPa}$ was measured at about $2 \mu \mathrm{m}$ indentation. For the epidermis, a reduced elastic modulus of $1.46 \pm 0.26 \mathrm{MPa}$ was measured at about $8 \mu \mathrm{m}$ indentation.

\section{Hydration}

Depth profiles of water content of the stratum corneum can be measured in vivo using confocal Raman spectroscopy. The moisture content of non-hydrated stratum corneum of the inner forearm can be seen to increase from below $30 \%$ at the outer surface to approximately $70 \%$ at a depth of $20 \mu \mathrm{m}$, showing a steep gradient from 5 to $15 \mu \mathrm{m}[24,25]$. Mechanical properties of the stratum corneum are strongly dependent on environmental temperature and relative humidity $(\mathrm{RH})$. Wildnauer et al. [21] carried out stress-strain measurements on isolated human stratum corneum samples which were kept at different relative humidities for 48 hours. Load-elongation curves were measured on $2 \mathrm{~mm}$ wide strips. Assuming a thickness of the stratum corneum of $20 \mu \mathrm{m}$, tensile elastic moduli can be calculated decreasing from about $4 \mathrm{GPa}$ at $32 \% \mathrm{RH}$ to $2 \mathrm{GPa}$ at $76 \% \mathrm{RH}$ and $1 \mathrm{GPa}$ at $98 \% \mathrm{RH}$. Using a tensile tester, Park and Baddiel [22] measured the tensile elastic modulus of $2 \mathrm{~mm}$ wide isolated stratum corneum strips as a function of relative humidity. The tensile elastic modulus was found to decrease from $2 \mathrm{GPa}$ at $30 \% \mathrm{RH}$ 




Figure 3. Tensile elastic modulus of isolated stratum corneum as a function of relative humidity. Data adapted from [21-23]. 
to $3 \mathrm{MPa}$ at $100 \% \mathrm{RH}$. Papir et al. [23] investigated the influence of relative humidity and temperature on the tensile properties of isolated newborn rat stratum corneum. A continuous decrease of the elastic modulus was observed with an increase in either relative humidity or temperature. In a semilogarithmic plot of the relative humidity versus tensile elastic modulus, two regimes can be observed separated by a clear discontinuity. At low relative humidity a moderate decline is observed from $8.87 \mathrm{GPa}$ at $26 \% \mathrm{RH}$ to $2.44 \mathrm{GPa}$ at $68 \% \mathrm{RH}$, where above $70 \% \mathrm{RH}$ an order of magnitude decrease can be seen to $12 \mathrm{MPa}$ at $100 \%$ RH. More recently, the influence of relative humidity on the mechanical properties of the stratum corneum has been discussed by $\mathrm{Wu}$ et al. [26]. They also report that their own inplane tensile measurements yielded moduli decreasing from approximately $1 \mathrm{GPa}$ to $5 \mathrm{MPa}$ with increasing hydration.

From Figure 3 it can be seen that the elasticity of the stratum corneum tends to decrease with increasing relative humidity. It is therefore important to consider temperature and humidity of the test environment when comparing the results from different experiments.

\section{Dermis and hypodermis}

At larger length scales, i.e. millimeters, the properties of the dermis and hypodermis determine the elastic behaviour of the whole skin. For example, Pailler-Mattei et al. [4] investigated the influence of the stratum corneum on the mechanical properties of in vivo human skin by successive removal of stratum corneum layers by tape-stripping. From indentation experiments carried out on the inner forearm using a $6.35 \mathrm{~mm}$ radius indenter, they found that the elastic modulus was about $7-8 \mathrm{kPa}$, independent of the thickness of the stratum corneum removed. They concluded that, at these length scales, the stratum corneum mechanical properties do not influence the global skin mechanical properties.

Delalleau et al. [13] calculated the skin elastic modulus by numerical analysis of results obtained from indentation of the human forearm using a $6.22 \mathrm{~mm}$ radius spherical indenter. Isotropic, linear elastic behaviour was assumed when analyzing the load-indentation curves. A reduced elastic modulus of $7.37 \mathrm{kPa}$ was measured at $600 \mu \mathrm{m}$ indentation. From numerical analysis of indentation curves measured on the inner forearm using a $6.35 \mathrm{~mm}$ radius indenter, Elleuch et al. [2] obtained a reduced elastic modulus of $15.4 \mathrm{kPa}$ at 80 $\mathrm{mN}$ normal load. It was observed that within the range of conditions tested, skin showed a dominant elastic behaviour.

Pailler-Mattei et al. [15] compared the elastic moduli measured on the inner forearm of two subjects different in age using a $6.35 \mathrm{~mm}$ radius steel indenter. For the 30-year-old subject a reduced elastic modulus of $8.8 \mathrm{kPa}$ was measured at $900 \mu \mathrm{m}$ indentation. At the same normal load for the 60-year-old subject, the reduced elastic modulus was $5.2 \mathrm{kPa}$ at $1400 \mu \mathrm{m}$. These results show a clear decrease of the elastic modulus with age. The same 
Table 1. Variation between subjects.

\begin{tabular}{llll}
\hline Study & Subjects & Age & Data elastic modulus \\
\hline$[3]$ & 4 males & $28-65$ years & $28.6 \pm 4 \mathrm{kPa}$ \\
& & & $24 \pm 3.8 \mathrm{kPa}$ \\
& 6 females & $28-65$ years & $13.7 \pm 5.3 \mathrm{kPa}$ \\
& & & $11.2 \pm 2.8 \mathrm{kPa}$ \\
{$[14]$} & 10 males & about 30 years & $18 \pm 4 \mathrm{kPa}$ \\
{$[15]$} & 1 female & 30 years & $8.8 \mathrm{kPa}$ \\
& 1 female & 60 years & $5.2 \mathrm{kPa}$ \\
{$[16]$} & 20 females & $55-70$ years & $8.3 \pm 1.74 \mathrm{kPa}$ \\
{$[18]$} & 3 females & $43 \pm 4$ years & $3.44 \pm 0.8 \mathrm{MPa}$ \\
& & & $1.46 \pm 0.26 \mathrm{MPa}$ \\
\hline
\end{tabular}

observations were made in a study by Zahouani et al. [16]. At $20 \mathrm{mN}$ normal load, the reduced elastic modulus decreases with age from $10.2 \mathrm{kPa}$ at 30 years to 7.5 and $5.3 \mathrm{kPa}$ at 60 and 80 years respectively.

\section{Variation between subjects}

Age and gender cause the effective elastic modulus to deviate from an average value. Besides age and gender, variation in measured mechanical properties between subjects arises from variation in skin properties such as thickness of the different skin layers or variation in aging. Differences in used test equipment, test conditions and the location on the inner forearm on which the measurements are carried out give further rise to the observed variation in results. Some studies report results obtained from a larger population of test results, so give insight in the variation of the measured elastic properties. From an extensive study of the load-displacement response of the forearm skin, Flynn et al. [27] observed a large variation within a population of 21 subjects between 21 and 52 years old. Analysis of the measurement data yielded a standard deviation of about $50 \%$. Jachowicz et al. [3] observed that mechanical properties changed with gender. Elastic moduli were measured on the forearm of 6 female and 4 male subjects between 28 and 65 years old using a $7.94 \mathrm{~mm}$ diameter spherical probe. The reduced elastic modulus was calculated by fitting the loading part of the load-indentation curve to Hertz theory. At normal loads of 0.06 and $0.1 \mathrm{~N}$, the average reduced elastic modulus was 13.7 and $11.2 \mathrm{kPa}$ for the female subjects, where for the male subjects 28.6 and $24 \mathrm{kPa}$ were measured.

The data reported in the studies described earlier were obtained from experiments carried out on subjects of different age and gender as shown in Table 1. From the results reported in the literature it is evident that the subject age $[15,16]$ and gender $[3]$ have a 
considerable influence on the measured elastic properties.

\section{Subcutaneous tissue}

With the length scale increasing further, the influence of underlying tissues such as muscles and bones becomes apparent. Pailler-Mattei et al. [14] measured the elastic properties of the skin as a function of indentation depth using a conical steel indenter with $45^{\circ}$ half angle and $10 \mathrm{~mm}$ height. Elastic moduli were calculated from the normal contact stiffness obtained from the initial part of the unloading curve. From about $1.5 \mathrm{~mm}$ depth, the elastic modulus increases with indentation depth from $12 \mathrm{kPa}$ to $18 \mathrm{kPa}$ at about $4 \mathrm{~mm}$. It was stated that this increase is due to the increasing contribution of subcutaneous tissues such as the muscles. From data found in the literature by Pailler-Mattei et al. [14], a reduced elastic modulus for muscle was calculated varying between 32 and $96 \mathrm{kPa}$, which is larger than the elasticity measured for whole skin. Below $1.5 \mathrm{~mm}$ indentation, the elastic modulus tends to increase with decreasing indentation.

In a pioneering study on the tribology of human skin, Johnson et al. [12] investigated the sliding contact of a glass probe on the inner forearm under different conditions. The mechanical response of the skin to normal loading was measured for a glass sphere with $8 \mathrm{~mm}$ radius of curvature. The loading curve followed a power law with an exponent of 1.44, very close to the Hertz equation (5). At $1 \mathrm{~N}$ normal load and $3 \mathrm{~mm}$ indentation, a reduced elastic modulus of $52.6 \mathrm{kPa}$ was calculated using Hertz's theory. Iivarinen et al. [28] obtained elastic moduli of the skin from numerical analysis of the results from experiments using a $2 \mathrm{~mm}$ diameter flat indenter. At rest, the elastic modulus for skin was $210 \mathrm{kPa}$, increasing to $446 \mathrm{kPa}$ and $651 \mathrm{kPa}$ under flexor and extensor loading of the muscle, respectively. Although in most studies the forearm is at rest during the experiments, in in vivo experiments on the volar forearm, the mechanical response at large length scales might be influenced by the tension of the underlying muscle.

\section{What value to use for the elastic modulus?}

To account for the multilayered and nonhomogeneous structure of the skin, as well as the relatively large deformations and any nonlinear effects in a relatively straightforward manner, it is suggested to use the effective elastic modulus $E_{e f f}$, sometimes referred to as the apparent or equivalent elastic modulus. Although it might formally not be correct from a physical point of view, the concept of an effective elastic modulus is very useful in contact and friction modelling as it reduces a multiparameter expression to a single parameter. The effective elastic modulus is a combination of the respective moduli of the skin layers and of the underlying tissue such as muscles and bones. The relative contribution of each 
individual skin layer to the effective elastic modulus is determined by the ratio of the thickness of the layer $t$ to the length scale of the contact $a$. Generally, the effective elastic modulus can be expressed as

$$
E_{\text {eff }}=E_{\text {substrate }}+\left(E_{\text {layer }}-E_{\text {substrate }}\right) \Phi(z)
$$

in which $\Phi$ is a weight function depending on the relative penetration $z$. The relative penetration $z$ being defined as the ratio of the indentation depth to the layer thickness $\delta / t$, or as the ratio of the length scale of the contact to the film thickness $a / t$, which is actually a relative length scale. Several methods to determine the relation between the effective modulus and the moduli of which it is composed can be found in the literature. An analytical solution to calculate the effective modulus of a layered material developed by Swain and Menčik [29], based on the work of Gao et al. [30], was used succesfully in the modelling of the contact between a rough surface and a layered material by Pasaribu and Schipper [31]. However, this model is valid only for modulus ratios up to 2. For a layered material such as the skin, the modulus ratios vary between 0.01 and 100, and the evolution of the effective elastic modulus with the ratio $a / t$ can be calculated following the numerical procedure of Perriot and Barthel [32]. Based on the calculated results, they presented an empirical function $E_{\text {eff }}^{*}(a / t)$ for a single-layer system. An analytical single-layer model developed by Bec et al. [33] was used to analyze the elastic behaviour of skin by PaillerMattei et al. [14], who described the effective elastic modulus of the skin by considering the skin as a soft elastic layer on a less compliant substrate, the muscle. To account for the relative contributions of the dermis and hypodermis they extended the Bec-model to a two-layer model. With this model, realistic values for the elastic moduli of the skin layers were obtained from the measured effective elastic modulus for relatively large length scales.

In the current study, the effects of the epidermis including the high modulus stratum corneum are incorporated by extending the two-layer model to a four-layer model. The global stiffness of the skin $K_{g}$ is given as the reciprocal sum of the stiffnesses of the different skin layers $K_{i}$ and of the underlying tissue $K_{n}$. From the definition of the stiffnesses $K_{i}=\pi a^{2} E_{i}^{*} / t$ and $K_{n}=2 E_{n}^{*} a$, the effective elastic modulus is given as

$$
\frac{1}{E_{e f f}^{*}}=2 \sum_{i=1}^{n-1} \frac{t_{i}}{f_{i}(a) \pi a E_{i}^{*}}+\frac{1}{f_{n}(a) E_{n}^{*}}
$$

To ensure correct boundary conditions, the polynomial functions $f_{i}(a)$ were introduced in the expressions of the different moduli $[14,33]$.

- When the contact size is very small as compared to the thickness of the upper layer, the 
effective elastic modulus equals the elastic modulus of the upper layer.

- If the properties of the different layers are the same as those of the substrate, the effective elastic modulus equals the elastic modulus of the substrate.

- If the properties of neighbouring layers are the same, those layers respond as one thick layer with the same elastic properties.

In analogy to the work of Bec et al. [33] and Pailler-Mattei et al. [14] for the four-layer model, this leads to the following expressions for the functions $f_{i}(a)$ :

$$
\begin{aligned}
f_{1}(a) & =1+\frac{2 t_{1}}{\pi a} \\
f_{i}(a) & =\left(1+\frac{2}{\pi a} \sum_{j=1}^{i-1} t_{j}\right)\left(1+\frac{2}{\pi a} \sum_{j=1}^{i} t_{j}\right), \text { for } i=2 \ldots n-1 \\
f_{n}(a) & =\left(1+\frac{2}{\pi a} \sum_{j=1}^{n-1} t_{j}\right)
\end{aligned}
$$

From Equations (11) and (12) it can be seen that the relation between the effective elastic modulus of the skin $E_{e f f}^{*}$ and the size of the contact $a$ depends solely on the respective thicknesses $t_{i}$ and elastic moduli $E_{i}^{*}$ of the different skin layers, indicated by the subscripts $i=1 \ldots 4$, and the underlying tissue indicated by the subscript $n=5$. Figure 4 gives a summary of the foregoing.



Figure 4. Schematic overview of the contact between a rough indenter and the skin simplified to the contact of a smooth spherical indenter and the skin. 
Table 2. Parameters used for calculating the skin effective elastic modulus: average values and ranges (between brackets).

\begin{tabular}{|c|c|c|c|c|}
\hline$\imath$ & Skin layer, tissue & $\begin{array}{l}\text { Elastic modulus } \\
E_{i}^{*}(\mathrm{MPa})\end{array}$ & $\begin{array}{l}\text { Thickness } \\
t_{i}(\mathrm{~mm})\end{array}$ & References \\
\hline 1 & $\begin{array}{ll}\text { Stratum corneum } & \text { dry } \\
& \text { wet }\end{array}$ & $\begin{array}{l}500(3.5-1000) \\
30(10-50)\end{array}$ & $0.025(0.01-0.04)$ & {$[4,17,18]$} \\
\hline 2 & Viable epidermis & 1.5 & $0.095(0.04-0.15)$ & {$[4,9,18]$} \\
\hline 3 & Dermis & $0.02\left(8-35 \times 10^{-3}\right)$ & $1.4(0.8-2)$ & {$[4,9,14,16]$} \\
\hline 4 & Hypodermis & $2 \times 10^{-3}$ & 0.8 & {$[14]$} \\
\hline 5 & Muscle & $0.25(0.1-0.4)$ & & {$[14,28]$} \\
\hline
\end{tabular}

\section{Skin properties}

For the properties of the different skin layers, a wide range of values are reported in the literature. The surface of the skin is formed by the stratum corneum, which is actually the outermost layer of the epidermis, the skin top layer. The thickness of the stratum corneum varies between 10 and $40 \mu \mathrm{m}[4,17,18]$. Values for the elastic modulus, measured from indentation experiments on isolated samples, vary from $3.5 \mathrm{MPa}$ to $2000 \mathrm{MPa}$ as shown in the paragraph Mechanical properties of the skin. For wet stratum corneum, the measured elastic modulus is considerably lower than for dry stratum corneum $[4,17,18]$. The viable epidermis has a thickness varying from 40 to $150 \mathrm{\mu m}[4,9]$ and an elastic modulus of about 1.5 MPa [18]. The second skin layer is the dermis, for which a thickness between 800 and $2000 \mu \mathrm{m}$ is reported [4, 14]. Elastic moduli are found ranging from 8.5 and $35 \mathrm{kPa}$ to $1.7 \mathrm{MPa}[14,16]$. Below the dermis lies the hypodermis, or subcutaneous fat layer. The thickness of the hypodermis varies considerably with anatomical site and between persons. At the volar forearm, the thickness and elastic modulus are approximately $800 \mu \mathrm{m}$ and 2 $\mathrm{kPa}$, respectively [14]. Finally, for the underlying muscle, the elastic modulus is found to vary between 100 and $400 \mathrm{kPa}$ [14, 28].

Table 2 shows the parameter values for layer thickness $t_{i}$ and elastic modulus $E_{i}^{*}$ that have been used to calculate the effective elastic modulus of the skin of the volar forearm $E_{e f f}^{*}$ as a function of contact length scale $a$. Average values were calculated from the ranges found in the literature.

\section{Results and discussion}

Figure 5 shows the effective elastic modulus as a function of length scale calculated using the data from Table 2. The complex behaviour of the skin of the volar forearm can be 
characterized by a single curve describing the elastic behaviour as a function of the length scale of the contact. As discussed in the paragraph Skin properties, different values for the respective layer thicknesses and elastic moduli can be found in the literature. The influence of the variation in these parameters on the effective elastic modulus has been indicated by dashed lines. The effective elastic modulus of the skin decreases several orders of magnitude when the length scale increases.

The evolution of the effective elastic modulus with contact length scale depicted in Figure 5 is the consequence of the rather complex anatomy of the skin. At different length scales different layers contribute to the apparent elasticity. The elastic moduli of the respective layers decrease with depth. With increasing length scale, the contribution of the lower and softer skin layers to the effective elastic modulus increases, its magnitude being determined by the ratio of elastically deformed volumes $a^{3} / t_{i} a^{2}$, or relative length scale $a / t_{i}$. Figure 6 plots the effective elastic modulus as a function of indentation depth $\delta$ for different indenter radii $R$ at indentations $\delta \leq 0.25 R$. At small indentation depths, the effective elastic modulus decreases with increasing indenter radius or, in other words, increasing elastically deformed volume due to the increasing contribution of the lower and softer skin layers. At larger indentation depths, the increasing contribution of the stiffer underlying tissue leads to an increase of the effective elastic modulus with increasing indenter radius.

\section{Stratum corneum and epidermis}

The stratum corneum serves a barrier function, preventing exogenous materials from penetrating into the body and vital components such as water from leaving the body [10]. The stratum corneum is the outermost layer of the viable epidermis which consists of keratinocytes, viable cells which are migrating towards the outer surface, eventually forming the stratum corneum. This top layer is composed of cornified dead cells, which have a diameter of 20-40 $\mathrm{mm}$ and a thickness of $1 \mu \mathrm{m}$. The amount of keratin, a tough structural protein, ranges from $30 \%$ at the base layer of the epidermis to $80 \%$ in the stratum corneum [9]. The corneocytes are held together by lipids and corneodesmosomes, protein structures acting as 'rivets'. The structure of the corneocytes being embedded in an intercellular lipid matrix is commonly referred to as a brick-and-mortar structure [34]. From a mechanical point of view, the corneocytes provide the stratum corneum with rigidity, whereas the lipids give it its flexibility.

Although, in the studies described in the paragraph Mechanical properties of the skin,

the elastic modulus is reported as a function of indentation depth, using Equations (8) and (9) the reported values can be plotted as a function of the length scale of the contact. 


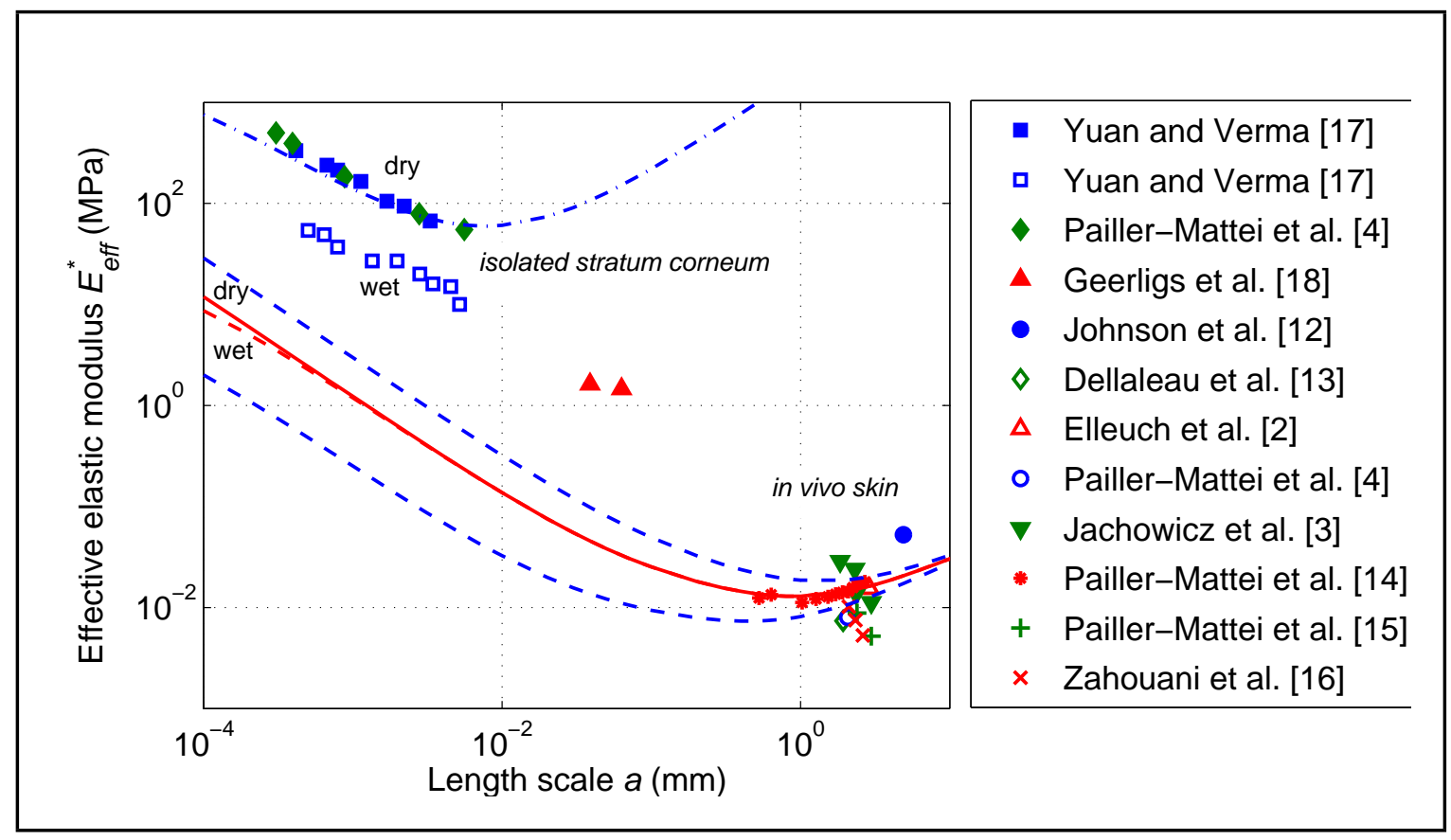

Figure 5. Effective elastic modulus of the skin of the volar forearm $E_{\text {eff }}^{*}$ as a function of length scale $a$ : calculated using average values (solid line), calculated using extreme values (dashed lines) and results from the literature [2-4, 12-18]. 


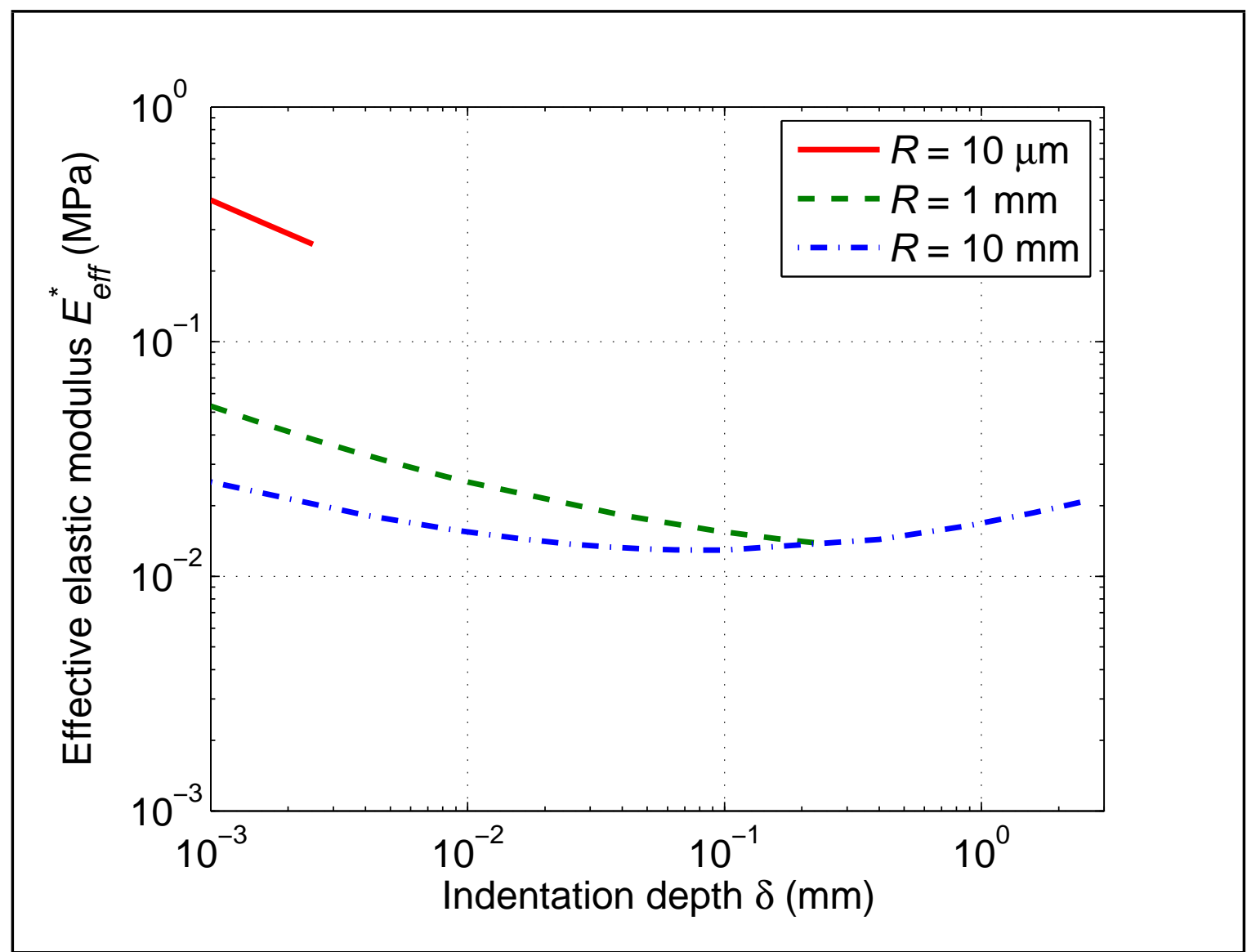

Figure 6. Effective elastic modulus of the skin of the volar forearm $E_{\text {eff }}^{*}$ as a function of indentation depth $\delta$ for different indenter radii $R$. 
The elastic properties from stratum corneum obtained from the literature are plotted as a function of contact length scale in Figure 5. Compared to the high elastic modulus measured for isolated stratum corneum, the calculated effective elastic modulus at small length scales is rather low. Perriot and Barthel [32] calculated equivalent elastic moduli as a function of relative length scale $a / t$ for substrate to layer elastic moduli ratios ranging from $10^{-2}$ to $10^{2}$. Their results show that the mechanical response of the layered system is dominated by the softer material, whether the softer material is the layer or the substrate, which explains the difference observed from Figure 5. The proposed model can be applied to a soft layer supported by a harder substrate, i.e. $E_{n}^{*}=70 \mathrm{GPa}$, as indicated by the dash-dotted line in Figure 5. Although it can be seen in Figure 5 that the elastic modulus of wet isolated stratum corneum is considerably lower than for dry stratum corneum [17], hydration has only a minor influence on the magnitude of the effective elastic modulus.

When the length scale is of the same order as the thickness of the stratum corneum, the influence of the epidermis and lower skin layers increases and the effective elastic modulus is found to decrease due to the lower elasticity of these layers.

\section{Dermis and hypodermis}

The dermis forms the second layer of the skin. The dermis houses several of the skin's appendages, such as the hair follicles, sweat and sebaceous glands and different nerve cells. Adhesion between the dermis and the epidermis is provided by the dermal-epidermal junction appearing like fingerlike projections providing mechanical strength by increasing the contact area and by mechanical interlocking. The dermis is the main mechanical component of the skin, maintaining spatial organization of the underlying tissues and protecting against mechanical stresses from the outside, so acting as a shock absorber. It is composed of collagen and elastin fibers in a viscous fluid, the extrafibrillar matrix. The collagen is the load-carrying element with intramolecular crosslinks giving strength to the dermis. The elastin provides the skin with its elasticity. With age, the density and orientation of these fibres change, leading to a considerable decrease in elasticity.

Underlying the dermis is the hypodermis, or subcutis, connecting the dermis to the underlying skeletal components. The hypodermis houses blood vessels and nerves. Furthermore, this subcutaneous fat layer provides energy storage, thermal insulation and is a cushion, protecting against mechanical shock. The thickness of the hypodermis is $1 \mathrm{~mm}$ to $5 \mathrm{~cm}$ and depends strongly on anatomical site and other factors such as a person's diet. The hypodermis is an extremely viscous and soft layer.

The results for the elastic moduli measured in vivo are plotted as a function of contact length scale in Figure 5. Although the model omits the nonlinear characteristics of the skin 
and does not take into account the presence of hair follicles, the different glands and blood vessels, the curve describes the evolution of the effective elastic modulus with the length scale of the contact rather well. Variation of properties of the lower and softer skin layers with age, gender or anatomical site give rise to the reported variations in elastic properties. Figure 7 shows the data reported earlier in Figures 1 and 5 for the reduced elastic modulus of in vivo human skin as a function of subject gender and age. With increasing age, the reduced elastic modulus at the larger length scales tends to decrease; for females, a lower elasticity is measured than for males. The variation in elastic properties can be attributed to a variation in elastic properties of the dermis.

After a decrease of several orders of magnitude with the length scale increasing from the micron to millimeter range, from a length scale of several millimeters a slight increase of the effective elastic modulus of the skin is caused by the underlying tissues such as muscle.

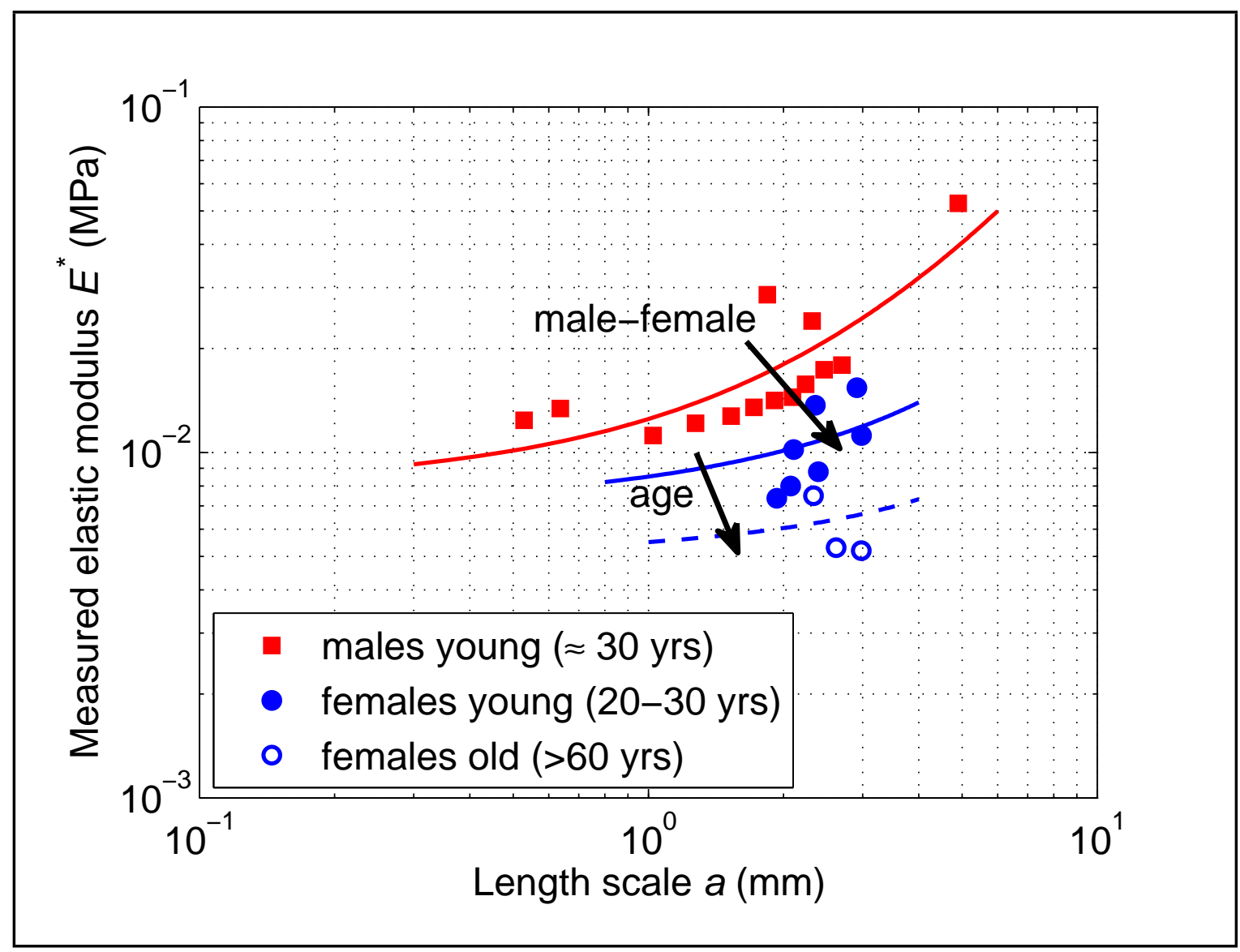

Figure 7. Measured elastic modulus of in vivo skin of the human volar forearm as a function of length scale. Data adapted from $[2-4,12-16]$. 
Paper B

\section{Contact and friction}

As shown in Equations (2) and (3), the friction force of a spherical probe sliding against the human skin is a function of the contact radius and the indentation depth. These contact parameters can be calculated iteratively from the indenter geometry and contact load using Hertz's Equations (4) and (5) as depicted schematically in Figure 8. The effective elastic modulus of the skin decreases with increasing length scale to a minimum value, after which a slight increase can be observed. Obviously, the evolution of the effective elastic modulus with the length scale of the contact leads to a deviation from the relation predicted by Hertz, which assumes a constant elastic modulus. With decreasing elastic modulus, the contact radius shows a stronger increase with increasing indenter radius and contact load. The indentation depth shows a stronger increase with increasing contact load and, as it is inversely proportional to the indenter radius, a weaker decrease with increasing indenter radius.

Although the solution provided by Hertz is based on several assumptions [see e.g. 5, 6], Hertz's theory is commonly used in all contact problems involving spherical bodies. Dintwa et al. [35] investigated the accuracy of the Hertz model under conditions in which some of these assumptions were violated using the finite element method. They found that friction between the contacting bodies (Hertz's theory assumes frictionless contacts) had no significant effect on the force-indentation relation predicted by Hertz, although the contact radius is slightly smaller [6]. Furthermore, no significant errors were found when the contact surface is not flat, as is the case with a rigid body indenting the softer skin. However, at large indentations, the Hertz model appears to underestimate the normal force, the error increasing with increasing indentation. At a ratio $\delta / R=0.025$ an error of $9.2 \%$ was found. They proposed an equation for the correction of the Hertz model.

For a layered material the relation between contact radius and indentation as shown in Equations (8) and (9), which are valid for homogeneous materials, breaks down. Perriot and Barthel [32] calculated the contact force and indentation as a function of contact radius for the elastic contact of an axisymmetric indenter and a coated substrate. For substrate to layer elastic moduli ratios ranging from $10^{-2}$ to $10^{2}$, equivalent elastic moduli were calculated. For a spherical indenter they showed that the relation between indentation and contact radius is given by $\delta=\left(a^{2} / R\right) \cdot \Phi(a / t)$ in which $\Phi(a / t)$ deviates from one when the contact radius $a$ approaches the layer thickness $t$. For soft layers on a harder substrate, as in the experiments on isolated stratum corneum attached to a glass or metal substrate, $\Phi<1$. For large moduli ratio this leads to a maximum error of around $30 \%$.

Thus the curve depicted in Figure 5 can be used to estimate contact parameters of a spherical probe sliding against the nonglabrous skin of the inner forearm with reasonable accuracy. When data for the thickness and elastic modulus of the individual skin layers 


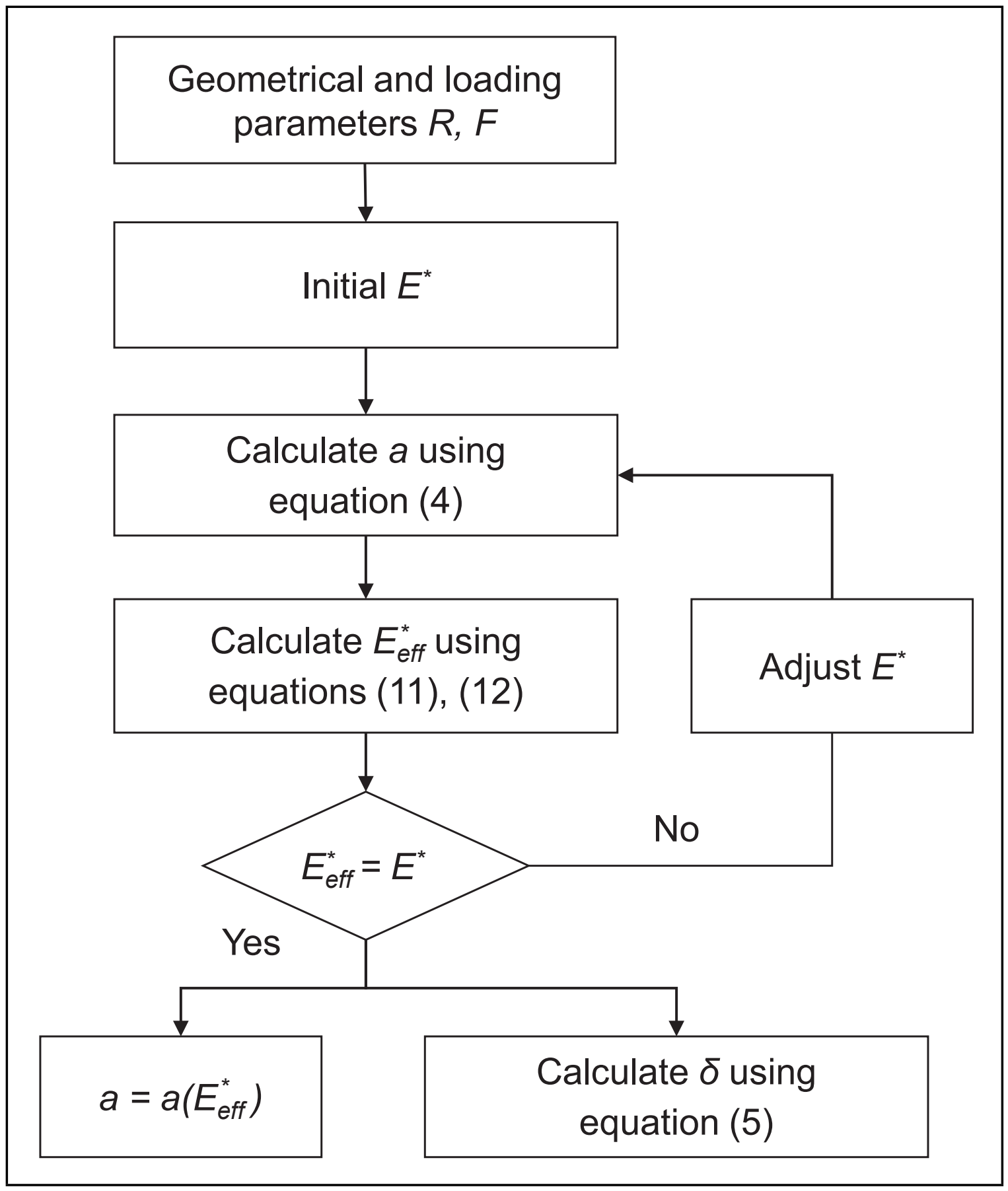

Figure 8. Algorithm for calculating the contact radius $a$ and indentation depth $\delta$ from the indenter radius $R$ and normal load $F$ taking into account the length scale effect of the effective elastic modulus $E_{\text {eff }}^{*}$ of the skin. 
are known, the Equations (11) and (12) can be used to predict the elastic behaviour at other anatomical sites. Contact models, such as the model proposed by Archard [36], can be used to incorporate the role of the surface topography of the nonglabrous, or hairy, skin in the contact and friction behaviour. However, describing the contact behaviour of the glabrous skin of, for example, the fingerpad requires a more complex model than the one presented in this study, which was based on the sphere-on-flat geometry. Although at first sight the fingertip seems to approximate a soft sphere, its contact behaviour is not that simple, as was shown by Derler et al. [37]. They found that at low loads the pressure distribution showed a round profile corresponding to the distribution predicted by Hertzian theory. At larger loads, however, the pressure distribution was conical, which was attributed to the influence of the bony structure underneath the softer tissues of the finger pad. The contact and friction behaviour is further complicated by the particular surface texture of the fingerpad skin, the contact area being determined by the number of fingerprint ridges rather than by deformation of the ridges [38].

\section{Conclusions}

Although an exact description of the mechanical behaviour of the skin requires an anisotropic, nonlinear, viscoelastic model, for contact modelling involving relatively small deformations, the mechanical behaviour can be described accurately by a single parameter: the effective elastic modulus. The complex behaviour of the skin of the volar forearm can be described using a closed-form expression $E=f(a)$ giving the elastic modulus $E$ as a function of the contact length scale $a$. Data collected from the literature shows that the measured elastic modulus of the skin varies orders of magnitude with varying contact length scale. The calculated effective elastic modulus of the skin describes the evolution of the elastic modulus with contact length scale very well. The length scale of the contact, which in the case of a spherical contact equals the contact radius $a$, is the parameter from which the effective elastic modulus is calculated. For a given load and geometry, geometrical parameters such as the contact radius and the indentation depth can be iteratively calculated using a set of analytical expressions.

The effective elastic modulus varies considerably with length scale. At the micro scale, for example when investigating the effect of textures or surface roughness, length scales are in the order of micrometers. The effective elastic modulus decreases several orders of magnitude with increasing length scale, from about $1 \mathrm{GPa}$ at the outer surface to $1 \mathrm{MPa}$ at a length scale of $1 \mu \mathrm{m}$. Although the elastic modulus of the stratum corneum decreases considerably with increasing hydration, there is only a minor influence of hydration on the magnitude of the effective elastic modulus. For indentation at the macro scale, for 
example in determining the apparent contact area in a tribological test, length scales are in the order of millimeters. The reduced elastic modulus is in the order of kilopascals, and varies with age and gender.

In this work a curve is presented, which describes the elastic response of the average inner forearm as a function of contact length scale. Because the expressions that were used to calculate the effective elastic modulus account for the elastic and geometrical properties of the skin layers, they can be used to predict the elastic behaviour at any other anatomical site for which these data are available. However, a study to confirm this was not conducted.

For the contact and friction modelling of human skin, a closed-form expression based on the anatomy of the skin exists, which yields the magnitude of the effective elastic modulus of the skin as a function of the length scale of the contact depending on variables such as age, gender and environmental conditions.

\section{Acknowledgements}

This research was carried out under project number M63.2.10409 in the framework of the Research Program of the Materials innovation institute M2i (www.m2i.nl).

\section{References}

1. H. Czichos. Tribology: A systems approach to the science and technology of friction, lubrication and wear. Elsevier, Amsterdam, 1978.

2. Khaled Elleuch, Riadh Elleuch, and Hassan Zahouani. Comparison of elastic and tactile behavior of human skin and elastomeric materials through tribological tests. Pol Eng Sci, 46(12):1715-1720, 2006.

3. J. Jachowicz, R. McMullen, and D. Prettypaul. Indentometric analysis of in vivo skin and comparison with artificial skin models. Skin Res Technol, 13(3):299-309, 2007.

4. C. Pailler-Mattei, S. Pavan, R. Vargiolu, F. Pirot, F. Falson, and H. Zahouani. Contribution of stratum corneum in determining bio-tribological properties of the human skin. Wear, 263(7-12):1038-1043, 2007.

5. H. Hertz. Über die Berührung fester elastischer Körper. J Reine Angewandte Math, 92:156-171, 1882.

6. K. L. Johnson. Contact mechanics. Cambridge University Press, Cambridge, 1999. 
7. C. P. Hendriks and S. E. Franklin. Influence of surface roughness, material and climate conditions on the friction of human skin. Tribol Lett, 37(2):361-373, 2009.

8. J. Van Kuilenburg, M.A. Masen, M.N.W. Groenendijk, V. Bana, and E. Van der Heide. An experimental study on the relation between surface texture and tactile friction. Tribol Int, 48:15-21, 2012.

9. Desmond J. Tobin. Biochemistry of human skin - our brain on the outside. Chem Soc Rev, 35(1):52-67, 2006.

10. K. Subramanyan, M. Misra, S. Mukherjee, and K. P. Ananthapadmanabhan. Advances in the materials science of skin: A composite structure with multiple functions. MRS Bulletin, 32(10):770-778, 2007.

11. J. A. Greenwood and J. B. P. Williamson. Contact of nominally flat surfaces. Proc $R$ Soc Lond A, 295(1442):300-319, 1966.

12. S.A. Johnson, D.M. Gorman, M.J. Adams, and B.J. Briscoe. The friction and lubrication of human stratum corneum. In C.M. Taylor D. Dowson, editor, Thin Films in Tribology, Proceedings of the 19th Leeds-Lyon Symposium on Tribology, held at the Institute of Tribology, University of Leeds, volume 25, pages 663-672. Elsevier, 1993.

13. Alexandre Delalleau, Gwendal Josse, Jean-Michel Lagarde, Hassan Zahouani, and Jean-Michel Bergheau. Characterization of the mechanical properties of skin by inverse analysis combined with the indentation test. J Biomech, 39(9):1603-1610, 2006.

14. C. Pailler-Mattei, S. Bec, and H. Zahouani. In vivo measurements of the elastic mechanical properties of human skin by indentation tests. Med Eng Phys, 30(5):599-606, 2008 .

15. C. Pailler-Mattei, S. Nicoli, F. Pirot, R. Vargiolu, and H. Zahouani. A new approach to describe the skin surface physical properties in vivo. Colloids Surf B, 68(2):200-206, 2009 .

16. H. Zahouani, C. Pailler-Mattei, B. Sohm, R. Vargiolu, V. Cenizo, and R. Debret. Characterization of the mechanical properties of a dermal equivalent compared with human skin in vivo by indentation and static friction tests. Skin Res Technol, 15(1):6876,2009 .

17. Yonghui Yuan and Ritu Verma. Measuring microelastic properties of stratum corneum. Colloids Surf B, 48(1):6-12, 2006. 
18. Marion Geerligs, Lambert van Breemen, Gerrit Peters, Paul Ackermans, Frank Baaijens, and Cees Oomens. In vitro indentation to determine the mechanical properties of epidermis. J Biomech, 44(6):1176-1181, 2011.

19. M. J. Adams, B. J. Briscoe, and S. A. Johnson. Friction and lubrication of human skin. Tribol Lett, 26(3):239-253, 2007.

20. W.C. Oliver and G.M. Pharr. An improved technique for determining hardness and elastic modulus using load and displacement sensing indentation experiments. J Mater Res, 7(06):1564-1583, 1992.

21. Richard H Wildnauer, James W Bothwell, and Alexander B Douglass. Stratum corneum biomechanical properties I. Influence of relative humidity on normal and extracted human stratum corneum. J Inv Dermatol, 56(1):72-78, 1971.

22. A. C. Park and C. B. Baddiel. Rheology of stratum corneum - I: A molecular interpretation of the stress-strain curve. J Soc Cosm Chem, 23:3-12, 1972.

23. Yoram S. Papir, Kuo-Hung Hsu, and Richard H. Wildnauer. The mechanical properties of stratum corneum: I. The effect of water and ambient temperature on the tensile properties of newborn rat stratum corneum. Biochim Biophys Acta, 399(1):170-180, 1975.

24. Mariko Egawa and Tomoko Kajikawa. Changes in the depth profile of water in the stratum corneum treated with water. Skin Res Technol, 15(2):242-249, 2009.

25. Noriaki Nakagawa, Masayuki Matsumoto, and Shingo Sakai. In vivo measurement of the water content in the dermis by confocal raman spectroscopy. Skin Res Technol, 16(2):137-141, 2010.

26. Kenneth S. Wu, William W. van Osdol, and Reinhold H. Dauskardt. Mechanical properties of human stratum corneum: Effects of temperature, hydration, and chemical treatment. Biomaterials, 27(5):785-795, 2006.

27. Cormac Flynn, Andrew Taberner, and Poul Nielsen. Measurement of the forcedisplacement response of in vivo human skin under a rich set of deformations. Med Eng Phys, 33(5):610-619, 2011.

28. Jarkko T. Iivarinen, Rami K. Korhonen, Petro Julkunen, and Jukka S. Jurvelin. Experimental and computational analysis of soft tissue stiffness in forearm using a manual indentation device. Med Eng Phys, 33:1245-1253, 2011. 
29. M.V. Swain and J. Menčik. Mechanical property characterization of thin films using spherical tipped indenters. Thin Solid Films, 253(1-2):204-211, 1994.

30. Huajian Gao, Cheng-Hsin Chiu, and Jin Lee. Elastic contact versus indentation modeling of multi-layered materials. Int J Sol Struct, 29(20):2471-2492, 1992.

31. H. R. Pasaribu and D. J. Schipper. Application of a deterministic contact model to analyze the contact of a rough surface against a flat layered surface. $J$ Tribol, 127(2):451, 2005.

32. A. Perriot and E. Barthel. Elastic contact to a coated half-space: Effective elastic modulus and real penetration. J Mater Res, 19(02):600-608, 2004.

33. S. Bec, A. Tonck, J. -M. Georges, E. Georges, and J. -L. Loubet. Improvements in the indentation method with a surface force apparatus. Phil Mag A, 74(5):1061-1072, 1996.

34. Kenneth S. Wu, William W. Van Osdol, and Reinhold H. Dauskardt. Mechanical and microstructural properties of stratum corneum. MRS Online Proceedings Library, 724:27-33, 2002.

35. Edward Dintwa, Engelbert Tijskens, and Herman Ramon. On the accuracy of the Hertz model to describe the normal contact of soft elastic spheres. Granular Matter, 10:209-221, 2007.

36. J. F. Archard. Elastic deformation and the laws of friction. Proceedings of the Royal Society of London. Series A. Mathematical and Physical Sciences, 243(1233):190 -205, 1957.

37. S. Derler, J. Süess, A. Rao, and G.-M. Rotaru. Influence of variations in the pressure distribution on the friction of the finger pad. Tribology International, (0), in press.

38. R. T. Spurr. Fingertip friction. Wear, 39(1):167-171, 1976.

\section{Appendix}

\section{Notation}

a contact radius, length scale $(\mathrm{m})$

A contact area $\left(\mathrm{m}^{2}\right)$ 
Paper B

$E \quad$ elastic modulus (Pa)

$E^{*} \quad$ reduced elastic modulus $(\mathrm{Pa})$

$E_{\text {eff }}^{*}$ effective elastic modulus $(\mathrm{Pa})$

$F \quad$ normal load $(\mathrm{N})$

$F_{\mu} \quad$ friction force (N)

$F_{\mu, a d h}$ friction force due to adhesive shear (N)

$F_{\mu, d e f}$ friction force due to deformation losses (N)

$R$ radius of curvature $(\mathrm{m})$

$t \quad$ layer thickness $(\mathrm{m})$

$z \quad$ relative penetration $(-)$

$\alpha \quad$ angle $\left(^{\circ}\right)$

$\delta \quad$ indentation depth, deformation (m)

$\beta \quad$ viscoelastic loss fraction (-)

$\nu \quad$ Poission ratio (-)

$\tau \quad$ shear strength of the interface $(\mathrm{Pa})$ 


\section{Paper C}

The role of the skin microrelief in the contact behaviour of human skin:

\section{Contact between the human finger and regular surface textures}

J. van Kuilenburg, M.A. Masen and E. van der Heide

Tribology International, Vol. 65, 2013, pp. 81-90

Special issue: 39th Leeds-Lyon Symposium on Tribology

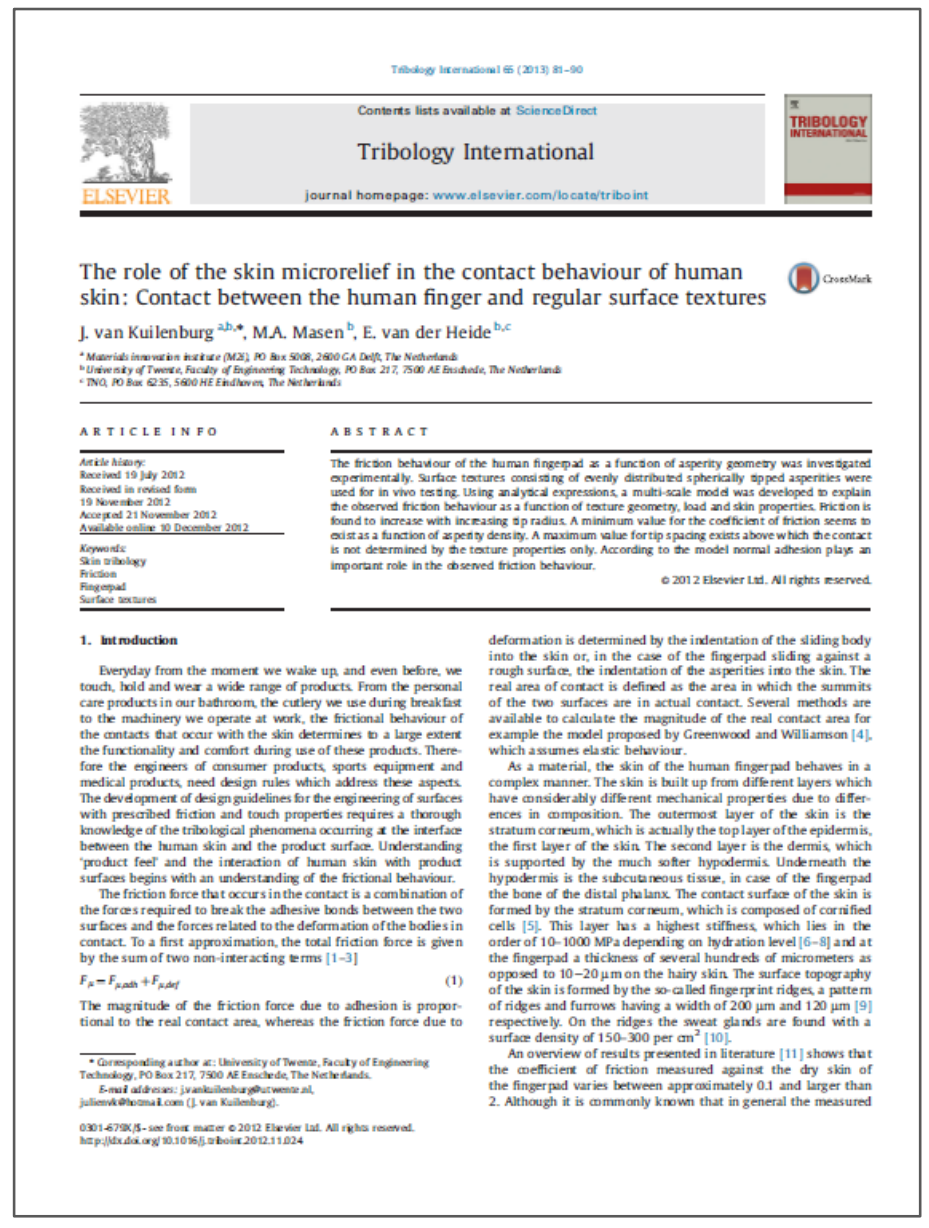





\title{
The role of the skin microrelief in the contact behaviour of human skin: Contact between the human finger and regular surface textures
}

\author{
J. van Kuilenburg, M.A. Masen and E. van der Heide
}

\begin{abstract}
The friction behaviour of the human fingerpad as a function of asperity geometry was investigated experimentally. Surface textures consisting of evenly distributed sphericallytipped asperities were used for in vivo testing. Using analytical expressions, a multiscale model was developed to explain the observed friction behaviour as a function of texture geometry, load and skin properties. Friction is found to increase with increasing tip radius. A minimum value for the coefficient of friction seems to exist as a function of asperity density. A maximum value for tip spacing exists above which the contact is not determined by the texture properties only. According to the model, normal adhesion plays an important role in the observed friction behaviour.
\end{abstract}

\section{Keywords}

Skin tribology, friction, contact mechanics, fingerpad, surface textures

\section{Introduction}

Every day from the moment we wake up, and even before, we touch, hold and wear a wide range of products. From the personal care products in our bathroom, the cutlery we use during breakfast to the machinery we operate at work, the frictional behaviour of contact that occurs with the skin determines to a large extent the functionality and comfort during use of these products. Therefore, the engineers of consumer products, sports equipment and medical products, need design rules which address these aspects. The development of design guidelines for the engineering of surfaces with prescribed friction and touch properties requires a thorough knowledge of the tribological phenomena occurring at the interface between the human skin and the product surface. Understanding 'product 
feel'and the interaction of human skin with product surfaces begins with an understanding of the frictional behaviour.

The friction force that occurs in the contact is a combination of the forces required to break the adhesive bonds between the two surfaces and the forces related to the deformation of the bodies in contact. To a first approximation, the total friction force is given by the sum of two non-interacting terms [1-3].

$$
F_{\mu}=F_{\mu, a d h}+F_{\mu, d e f}
$$

The magnitude of the friction force due to adhesion is proportional to the real contact area, whereas the friction force due to deformation is determined by the indentation of the sliding body into the skin or, in the case of the fingerpad sliding against a rough surface, the indentation of the asperities into the skin. The real area of contact is defined as the area in which the summits of the two surfaces are in actual contact. Several methods are available to calculate the magnitude of the real contact area such as, for example, the model proposed by Greenwood and Williamson [4], which assumes elastic behaviour.

As a material, the skin of the human fingerpad behaves in a complex manner. The skin is built up from different layers which have considerably different mechanical properties due to differences in composition. The outermost layer of the skin is the stratum corneum, which is actually the top layer of the epidermis, the first layer of the skin. The second layer is the dermis, which is supported by the much softer hypodermis. Underneath the hypodermis is the subcutaneous tissue, in the case of the fingerpad the bone of the distal phalanx. The contact surface of the skin is formed by the stratum corneum, which is composed of cornified cells. [5] This layer has the highest stiffness, which lies in the order of 10 to $1000 \mathrm{MPa}$ depending on hydration level [6-8], and at the fingerpad a thickness of several hundreds of micrometers as opposed to 10-20 $\mathrm{mm}$ on the hairy skin. The surface topography of the skin is formed by the so-called fingerprint ridges, a pattern of ridges and furrows having a width of $200 \mu \mathrm{m}$ and $120 \mu \mathrm{m}$ [9] respectively. On the ridges the sweat glands are found with a surface density of 150-300 per $\mathrm{cm}^{2}$ [10].

An overview of results presented in the literature [11] shows that the coefficient of friction measured against the dry skin of the fingerpad varies between approximately 0.1 and larger than 2. Although it is commonly known that, in general, the measured coefficient of friction is dependent on factors such as environmental conditions and measurement method, the frictional behaviour of the fingerpad is also influenced by the inherent properties of the finger, which vary considerably among persons. Several studies show that, as a function of normal load, the measured friction force follows a power law with exponents ranging between 0.62 and 0.89 [10, 12-15]. The evolution of the friction force with 
normal load can be explained partly by the growth of the apparent contact area, which follows a power law with exponents between 0.2 and 0.77 [12, 13, 15-18]. When in contact with a smooth surface, the real contact area of the fingerpad is formed by the fingerprint ridges. Soneda and Nakano [17] measured the apparent contact area and real contact area against smooth glass. For the apparent contact area exponents between 0.478 and 0.641 were measured as a function of normal load, whereas for the real contact area exponents between 0.583 and 0.806 were obtained. It thus appears that the real contact area grows slightly faster with normal load than does the apparent contact area, which was confirmed by other authors [19-21]. Comparing the different studies shows that the magnitude of the measured contact area at constant load might vary by a factor two between persons, which is mainly due to the aforementioned variation in geometrical and mechanical properties of the fingertip. When sliding against a rough surface, the friction can be seen to decrease with roughness amplitude as reported by several authors [10, 22-25], which can be attributed to the real area of contact decreasing with increasing roughness. From a certain point, when the roughness amplitude increases further, increased ploughing leads to an increase of the friction as reported by Tomlinson et al. [26]. Although the observed trends in the different studies are consistent, it is the surface geometry rather than the surface roughness amplitude that determines the friction behaviour. Gee et al. [23] showed a considerable difference in measured friction as a function of finishing method for surfaces having the same $R_{a}$-roughness.

In this work, the contact between an asperity and the skin of the fingerpad was investigated. When developing a contact model which predicts the area and indentation depth of the surface asperities in contact with the fingerpad, several difficulties are encountered. An essential input parameter in such a model is the elastic modulus, which is highly scaledependent due to the layered composition of the skin. Furthermore, the surface topography of the fingerpad formed by the fingerprint ridges hinders the use of statistical models to calculate the real area of contact. In vivo experimental validation of the contact model at the level of a single surface asperity is deemed impossible. Therefore, to investigate the friction behaviour as a function of asperity geometry, surface textures consisting of evenly distributed spherically-tipped asperities were produced using ultra-short pulsed laser technology. Analytical models available from contact mechanics theory were used to explain the observed friction behaviour of different textures sliding against a polymeric sphere in a model experiment. These models were extended to study the friction behaviour of the fingerpad measured in in vivo experiments. 


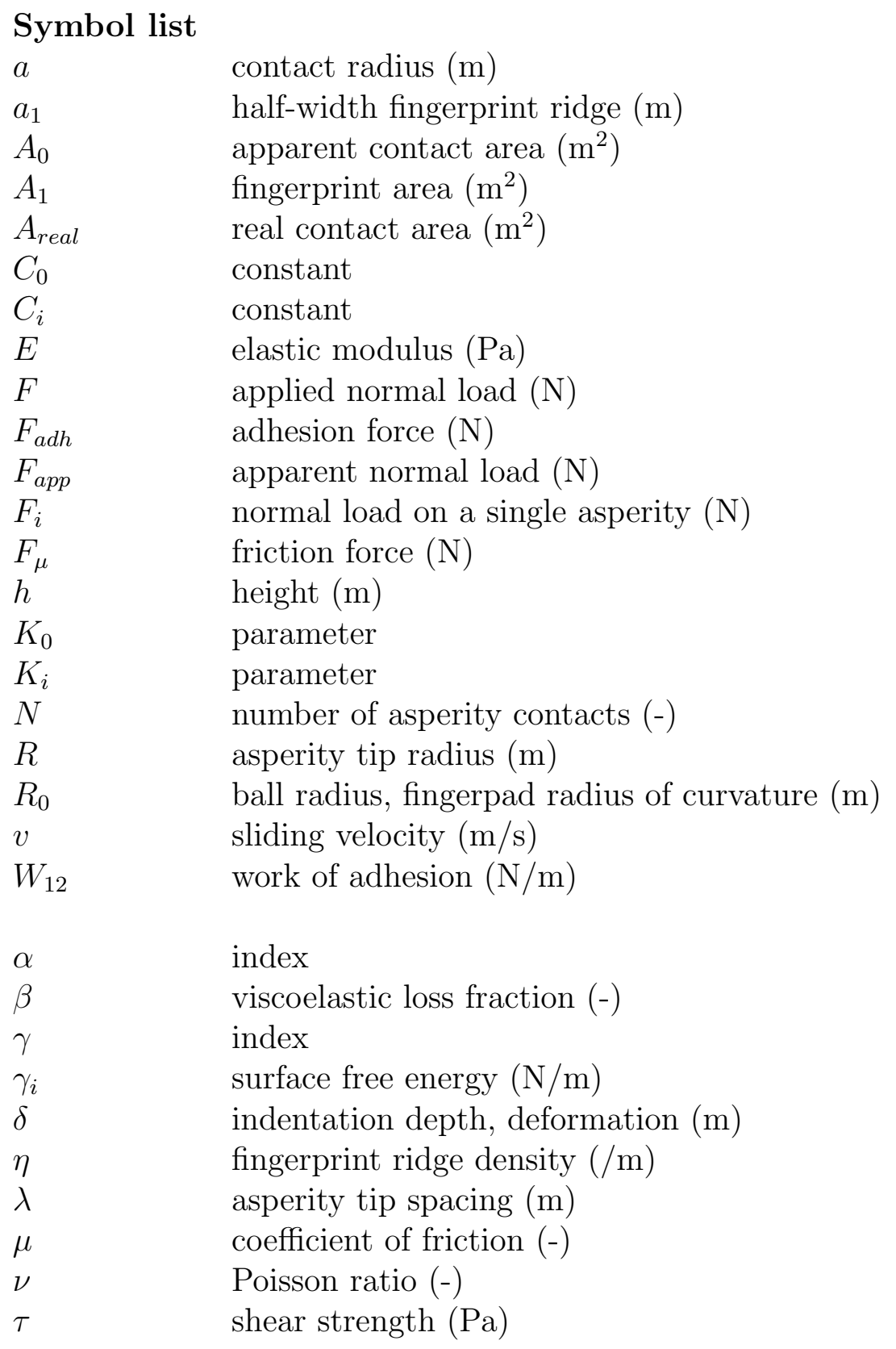




\section{Model system}

\subsection{Ball-on-disk experiments}

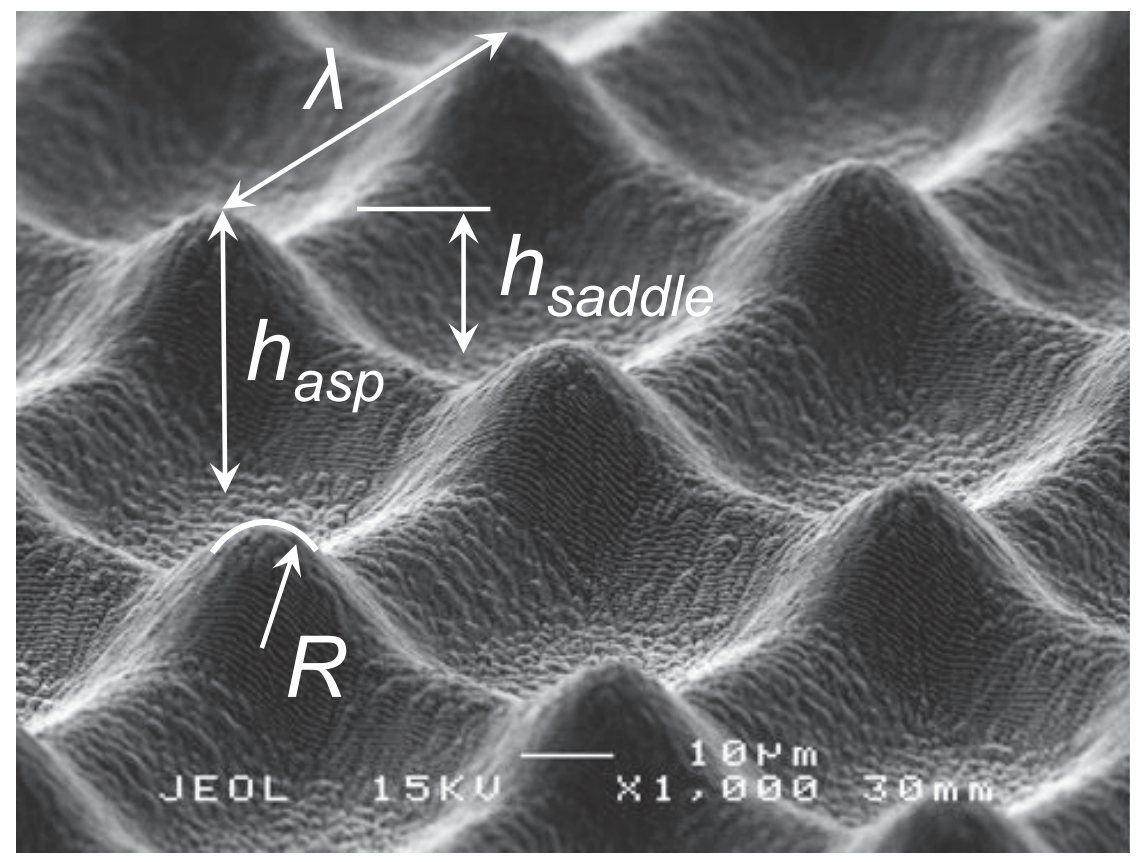

Figure 1. SEM image of a laser-textured sample with tip radius $R=5 \mu \mathrm{m}$ and spacing $\lambda=50 \mu \mathrm{m}$ (courtesy of Lightmotif, the Netherlands).

The frictional behaviour of two surfaces that are in sliding contact depends on the combination of the two contacting materials, their surfaces and micro-geometry, any lubricants and the environmental conditions, as well as the operational conditions of the contact [27]. As the friction of skin is known to vary considerably with environmental conditions, bodily state and time [22], to reduce the number of variables influencing the measured friction behaviour, a first series of model experiments was carried out using a ball-on-disk set-up. In several tribological studies polymers, such as TPU, PUR and silicone-based materials, are used as a substitute material to simulate the mechanical behaviour of the whole skin [28-31]. Because the stratum corneum in its dry state has a much higher elastic modulus than the whole skin [8], in this study polypropylene (PP) with an elastic modulus of 1.5 GPa was choosen as a countersurface material.

Surface textures consisting of evenly distributed spherically-tipped asperities were produced using ultra-short pulsed laser technology (Lightmotif, the Netherlands). The textures were applied onto flat stainless steel samples measuring 12 by $12 \mathrm{~mm}$. Figure 1 shows a SEM image of a representative sample. The surface textures are composed from 
bumps having a spherical tip with radius $R$, which are evenly distributed with a spacing $\lambda$ between the tips. A series of 16 samples was produced with tip radii of $2,5,10$ and 20 $\mu \mathrm{m}$ and spacings varying between 10 and $200 \mu \mathrm{m}$. The height of the asperities is $20 \pm 2$ $\mu \mathrm{m}$, the height of the saddle points is $10 \pm 2 \mu \mathrm{m}$. Surface topography was measured using a 3D Laser Confocal Microscope model VK-9700 (Keyence, USA). Tip radii of the asperities were obtained from line scans of the 3D surface profile. The asperities have a conical shape with a spherical tip, as can be seen from Fig. 1. The height of the spherical geometry, measured from the top, is approximately 2, 3, 7 and $8 \mu \mathrm{m}$ for respectively the 2, 5, 10 and $20 \mu \mathrm{m}$ radius tips. All samples were cleaned ultrasonically using ethanol before and in between the experiments.

Sliding friction between a polypropylene ball (6 mm diameter, ground) and textured samples was measured using a pin-on-disk tribometer (Nanotribometer, CSM, Switzerland). The tribometer was equipped with a force sensor (type ST-051) which measures normal load in the range $0.2-600 \mathrm{mN}$ and lateral (friction) loads in the range $0.3-1000 \mathrm{mN}$. Measurements were carried out at $0.1 \mathrm{~N}$ normal load and $2 \mathrm{~mm} / \mathrm{s}$ sliding velocity at a 10 $\mathrm{mm}$ track diameter. The coefficient of friction $\mu$ was calculated as the ratio of the measured normal load and the tangential load. The average of 3 revolutions was taken as the coefficient of friction $\mu$ of a single measurement. No running-in behaviour was observed during the $94 \mathrm{~mm}$ sliding distance. After the experiments the PP balls were inspected visually. No wear was observed. All tests were carried out at a room temperature of $20 \pm 2^{\circ} \mathrm{C}$ and relative humidity $45 \pm 5 \%$.

\subsection{Contact model}

Adopting the two-term friction model, for the polypropylene ball sliding against a regular surface texture, the first term in Eq. (1) can be calculated from

$$
F_{\mu, a d h}=\tau A_{\text {real }}
$$

in which $\tau$ is the shear strength of the interface and $A_{\text {real }}$ is real contact area. When the surface asperities indenting the softer polymer are moved forward, work is dissipated due to the viscoelastic nature of the material. The deformation component equals the amount of work lost by viscoelastic hysteresis per unit sliding distance thus being proportional to a viscoelastic loss fraction $\beta$ and the relative indentation of the asperities $\delta / a=a / R$ into the polypropylene [32].

$$
F_{\mu, d e f}=\frac{3}{16} \beta \frac{a}{R} F
$$


For an elastic contact, the contact radius $a$ and indentation depth or deformation $\delta$ can be calculated from the applied normal load $F$ and radius of curvature of the body $R$ using Hertz's theory [33, 34] as shown in Eqs. (4) and (5) respectively.

$$
\begin{aligned}
a_{\mathrm{H}} & =\left(\frac{3}{4} \frac{R F}{E^{*}}\right)^{1 / 3} \\
\delta_{\mathrm{H}} & =\left(\frac{9}{16} \frac{F^{2}}{R E^{* 2}}\right)^{1 / 3}
\end{aligned}
$$

In which $E^{*}$ represents the reduced elastic modulus, defined as

$$
\frac{1}{E^{*}}=\frac{1-\nu_{1}^{2}}{E_{1}}+\frac{1-\nu_{2}^{2}}{E_{2}}
$$

with $E_{1}$ and $E_{2}$ the respective elastic moduli and $\nu_{1}$ and $\nu_{2}$ the Poisson ratios of the contacting materials. Although the solution provided by Hertz is based on several assumptions [see e.g. 33, 34], Hertz's theory is commonly used in all contact problems involving spherical bodies. Dintwa et al. [35] investigated the accuracy of the Hertz model under conditions in which some of the assumptions were violated using the finite element method. They found no significant errors when the contact surface is not smooth. However, at large indentations, the Hertz model appears to overestimate the indentation depth at given normal load, the error increasing with increasing indentation. At a ratio $\delta / R=0.025$ an error of $9.2 \%$ was found.

The apparent contact area between a ball with radius $R_{0}$ and a textured surface can be calculated as $A_{0}=\pi a_{0}^{2}$ in which $a_{0}$ is given by Eq. (4). The contact area and indentation depth of each individual asperity can be calculated from the normal load $F$ acting on this asperity. Since the asperities are arranged in a regular pattern having a spacing $\lambda$, the number of surface features $N$ in contact with the ball can be calculated from the contact area $A_{0}$ following

$$
A_{0}=N \lambda^{2}
$$

so that the normal load acting on each individual surface asperity $F_{i}$ can be calculated from

$$
F=N F_{i}
$$

The contact area and indentation of each individual asperity can now be calculated from Eqs. (4) and (5) using Hertz's theory. However, at high asperity densities the applied 
normal load on each individual asperity decreases, so that the force exerted by normal adhesion between the two surfaces will play a role [36]. The contact theory developed by Johnson, Kendall and Roberts [37] takes into account the surface forces due to adhesion. The JKR theory is applied to contacts of large, soft bodies with high surface energies. The adhesive force acting on a rigid asperity with tip radius $R$ equals the normal force necessary to separate the two surfaces and is given as

$$
F_{a d h, i}=\frac{3}{2} \pi R W_{12}
$$

The work of adhesion $W_{12}$ can be measured from indentation experiments or be approximated from the surface energy $\gamma_{i}$ of the materials [38] following

$$
W_{12} \approx 2 \sqrt{\gamma_{1} \gamma_{2}}
$$

From Eq. (9) it can be seen that the adhesive force acting on an asperity is independent of the elastic properties and the applied normal load. The apparent normal force acting on the asperity is given by [37] as

$$
F_{a p p, i}=F_{i}+2 F_{a d h, i}+2 \sqrt{F_{a d h, i}\left(F_{i}+F_{a d h, i}\right)}
$$

where $F_{i}$ is the externally applied normal load, so that for an asperity with radius $R$, the contact radius $a_{i}$ and indentation depth $\delta_{i}$ are given by

$$
\begin{aligned}
a_{i} & =\sqrt[3]{\frac{3}{4} \frac{R F_{a p p, i}}{E^{*}}} \\
\delta_{i} & =\frac{a_{i}^{2}}{3 R}\left(1+2 \frac{F_{i}}{F_{a p p, i}}\right)
\end{aligned}
$$

Due to the additional load on the contact, the radius of the adhesive contact is larger than the radius of the non-adhesive contact. From the contact radius $a_{i}$ the real area of contact between the ball and the surface texture can be approximated using

$$
A_{\text {real }}=\pi a_{i}^{2} N
$$

so that the contact ratio and relative indentation can be expressed as, respectively

$$
\frac{A_{\text {real }}}{A_{0}}=\pi\left(\frac{3}{4 E^{*}}\right)^{\frac{2}{3}}\left(\frac{R}{\lambda}\right)^{\frac{2}{3}}\left(\frac{F_{a p p}}{A_{0}}\right)^{\frac{2}{3}}
$$


and

$$
\frac{a_{i}}{R}=\left(\frac{3}{4 E^{*}}\right)^{\frac{1}{3}}\left(\frac{\lambda}{R}\right)^{\frac{2}{3}}\left(\frac{F_{a p p}}{A_{0}}\right)^{\frac{1}{3}}
$$

\section{$2.3 \quad$ Results}

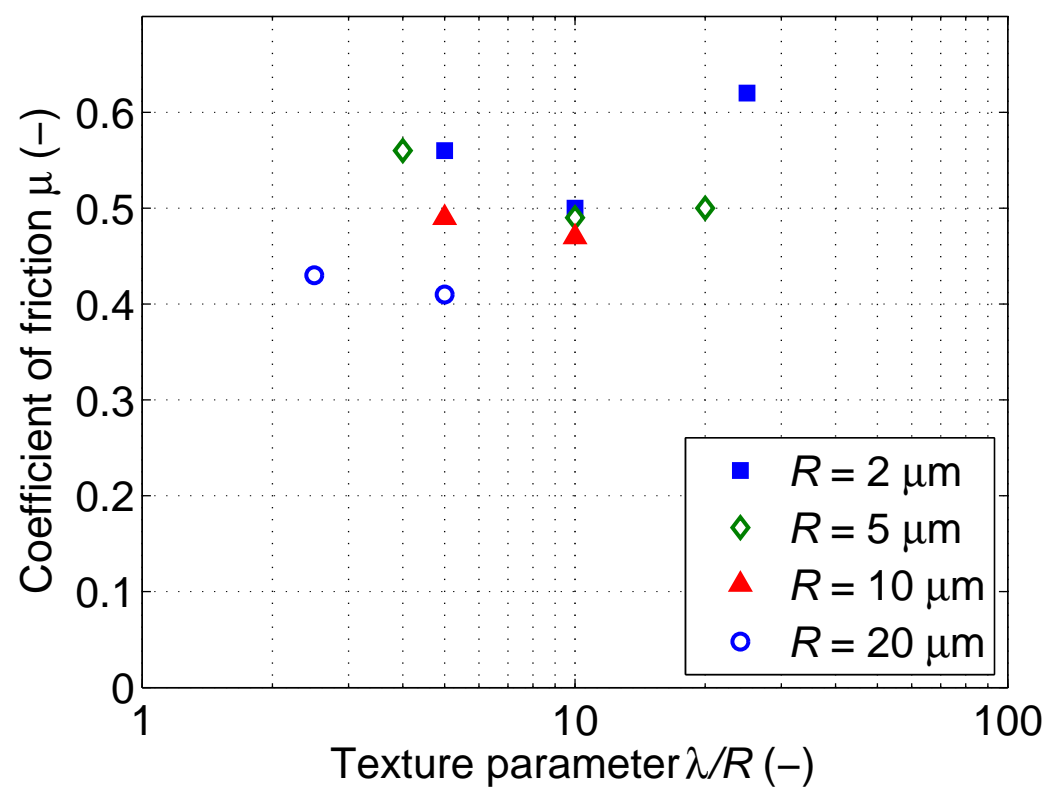

Figure 2. Measured coefficient of friction as a function of dimensionless spacing $\lambda / R$ for different tip radii $R$ measured against a polypropylene ball $(\varnothing=6 \mathrm{~mm})$ at $F=0.1 \mathrm{~N}$ and $v=2 \mathrm{~mm} / \mathrm{s}$.

Figure 2 shows the coefficient of friction between a polypropylene ball and different surface textures as a function of dimensionless tip spacing $\lambda / R$. Figure 3 shows the contact ratio and relative indentation as a function of dimensionless spacing $\lambda / R$ for different asperity tip radii calculated using Eqs. (15) and (16) and the data summarized in Table 1. Comparing Figs. 2 and 3 shows that trends observed in the experiments can be explained from the contact model. For low values of tip spacing the friction behaviour is determined mainly by the adhesion component, thus by the magnitude of the real contact area. The measured coefficient of friction as well as the predicted real contact area decrease with increasing spacing. The dependence of the evolution of both the measured coefficient of friction and the calculated contact area on the tip radius can be attributed to normal adhesion. The calculations show that with increasing spacing the normal load on a single 

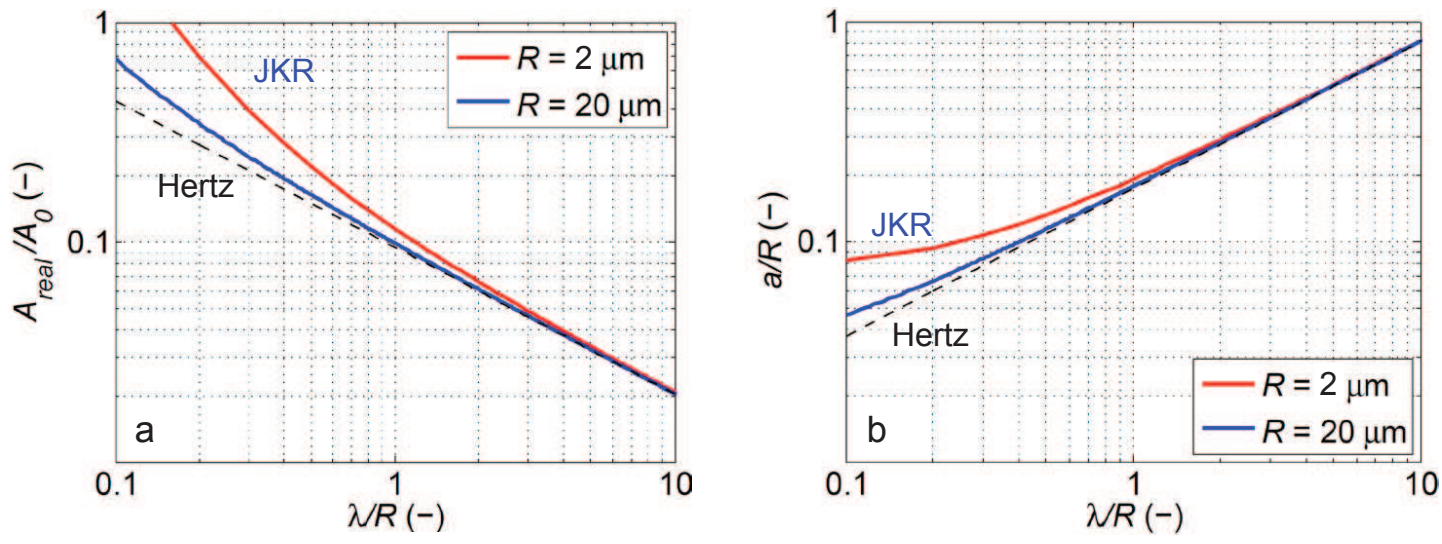

Figure 3. Calculated contact ratio $A_{\text {real }} / A_{0}$ (a) and relative indentation $a / R(\mathrm{~b})$ as a function of dimensionless spacing $\lambda / R$ for different tip radii $R$.

Table 1. Parameter values used in calculations

\begin{tabular}{ll}
\hline Parameter & Value \\
\hline Elastic modulus steel $E_{1}$ & $210 \mathrm{GPa}$ \\
Poisson ratio steel $\nu_{1}$ & 0.3 \\
Elastic modulus PP $E_{2}$ & $1.5 \mathrm{GPa}$ \\
Poisson ratio PP $\nu_{2}$ & 0.45 \\
Ball radius $R_{0}$ & $3 \mathrm{~mm}$ \\
Applied normal load $F$ & $0.1 \mathrm{~N}$ \\
Work of adhesion $W_{12}$ & $60 \mathrm{mN} / \mathrm{m}$ \\
\hline
\end{tabular}


Table 2. Characteristics of the textured samples used in in vivo experiments

\begin{tabular}{ccccc}
\hline$R(\mu \mathrm{m})$ & $\lambda(\mu \mathrm{m})$ & $\lambda / R(-)$ & $h_{\text {asp }}(\mu \mathrm{m})$ & $h_{\text {saddle }}(\mu \mathrm{m})$ \\
\hline 2 & 20 & 10 & 18 & 9 \\
5 & 20 & 4 & 18 & 9 \\
5 & 50 & 10 & 22 & 12 \\
5 & 100 & 20 & 24 & 12 \\
10 & 100 & 10 & 24 & 12 \\
20 & 200 & 10 & 25 & 11 \\
\hline
\end{tabular}

asperity increases, which leads to a decrease in the relative contribution of the load due to normal adhesion. When the contribution of normal adhesion decreases the curves converge to the Hertz solution, shown in Fig. 3 as a dotted line. Figure 2 shows that after decreasing to a minimum value the coefficient of friction increases with increasing spacing. The contribution of the deformation component of friction becomes apparent, which is proportional to the relative indentation of the asperities. The indentation increases with increasing spacing as shown in Fig. 3.

From the friction results depicted in Fig. 2 the viscoelastic loss modulus $\beta$ and surface shear strength $\tau$ can be approximated using Eqs. (15) and (16). With $\beta$ varying between 0 and 0.5 the shear strength has a value in the order of 250-300 MPa.

It can be concluded that the two-term friction model in combination with basic contact models can be used to explain the observed friction behaviour. Within the range of tested parameters, the relative contributions of the adhesion and the deformation component can be distinguished. A selection of texture parameters was made for in vivo testing.

\section{Fingerpad contact}

\subsection{In vivo experiments}

A second series of six samples was produced for in vivo friction experiments. Table 2 summarizes the characteristics of the textured samples. Sliding friction between the fingerpad and textured samples was measured using a load cell model ATI Gamma (ATI Industrial Automation, USA) as shown in Fig. 4. Resolution of the 3 -axis force/torque sensor is 25 $\mathrm{mN}$ in the normal direction and $12.5 \mathrm{mN}$ in tangential direction, with a sample frequency of $100 \mathrm{~Hz}$. Textured samples measuring 40 by $10 \mathrm{~mm}$ were attached to the load cell using double- sided adhesive tape. In vivo experiments were carried out using the fingerpad of the left (dominant) index finger of a 37-year-old male subject. Measurements were carried 
out by sliding the index finger over the sample. The finger is moved three times back and forth in the horizontal plane. Measurements were carried out at normal loads around 1 and $2 \mathrm{~N}$. The normal load was applied by attaching dead weights of $100 \mathrm{~g}$ and $200 \mathrm{~g}$ respectively to the finger using double-sided adhesive tape. All tests were carried out at a room temperature of $20 \pm 2^{\circ} \mathrm{C}$ and relative humidity $45 \pm 5 \%$. Before the experiments the hands were washed using water and soap and air dried. After a rest period of approximately 10 min. to allow the washed skin to regain its normal condition the experiments were carried out. Before and during the measurements the hydration level of the skin surface was monitored using a Corneometer CM 825 (Courage+Khazaka GmbH, Germany). The Corneometer measures the changing capacitance of a precision capacitor in arbitrary units $\mathrm{AU}$, which represent the change in the dielectric constant due to hydration of the skin surface. The measurement depth is $10-20 \mu \mathrm{m}$. The Corneometer is an established equipment for measuring hydration in skin tribology studies, [see e.g. 39-41].

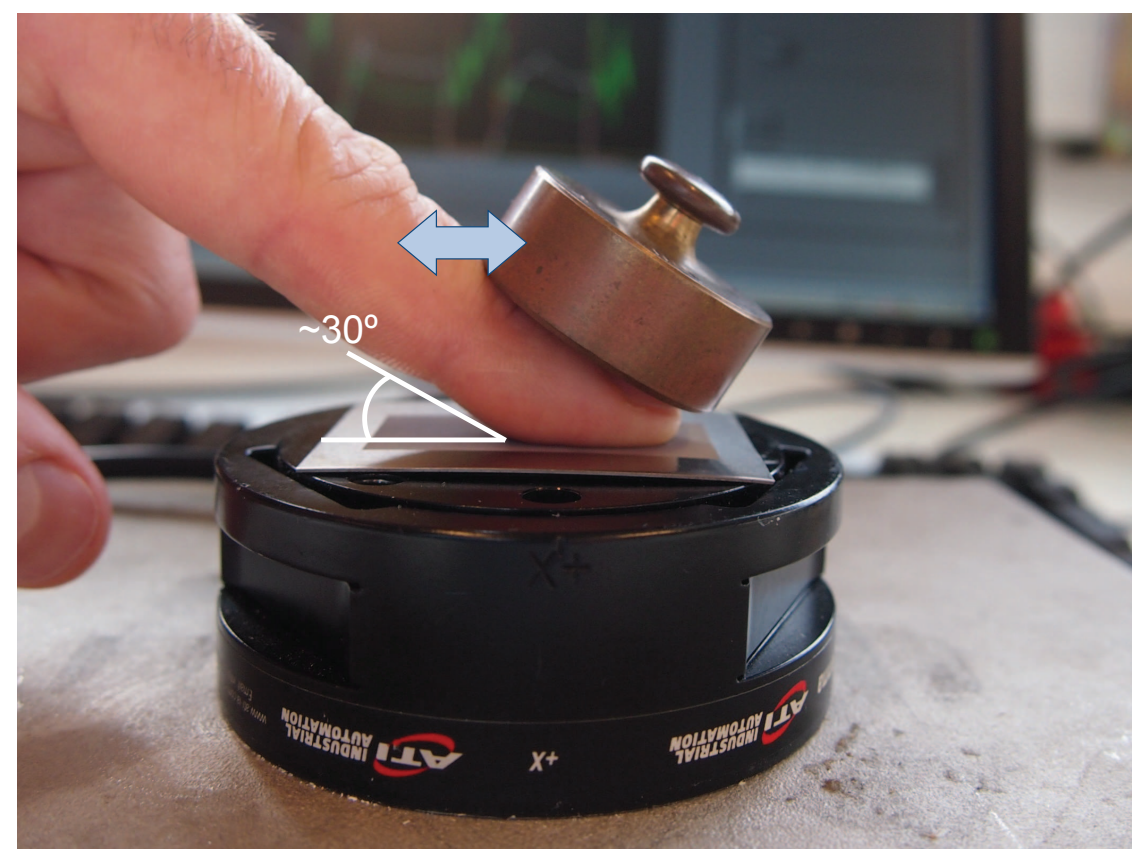

Figure 4. Experimental set-up for measuring the sliding friction between the fingerpad and textured samples.

\subsection{Contact model}

To investigate the friction behaviour observed in the experiments the two-term model Eq. (1) was adopted. Several authors [11, 42] argue that in the sliding contact between 
a rough surface and the skin ploughing or interlocking terms contribute to the friction. Since the spacing between the asperities is smaller than the width of the fingerprint ridges, interlocking was not considered in this study. Bowden et al. [43] showed that the ploughing term is proportional to the relative indentation of the asperities as is the hysteresis term given in Eq. 3, so that the contact parameters of interest are the contact ratio $A_{\text {real }} / A_{0}$ and the relative indentation of the asperities into the skin $a_{i} / R$.

macro scale

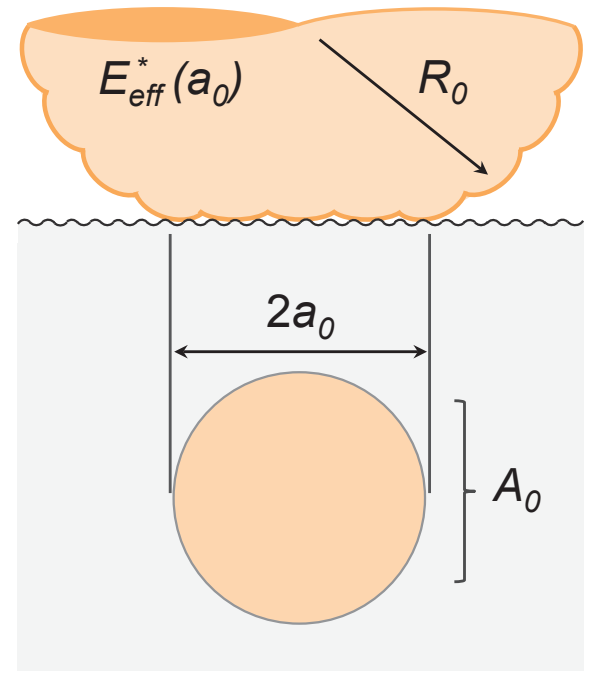

meso scale

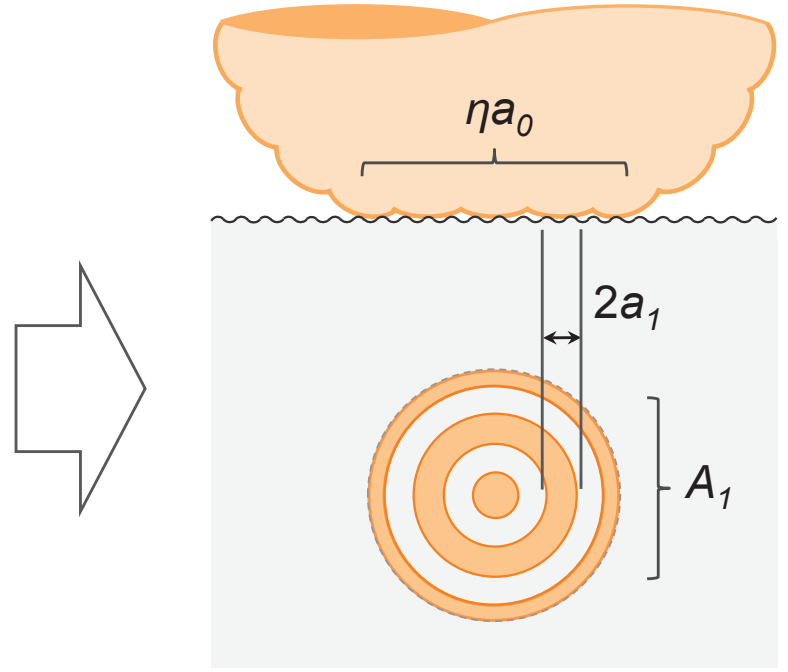

Figure 5. Schematic overview of the contact dimensions of the contact formed by the fingerpad (macro scale) and the fingerprint ridges (meso scale).

In the case of contact between skin and a much stiffer counterbody, $E_{\text {counterbody }} \gg E_{\text {skin }}$ and the reduced elastic modulus depends solely on the properties of the skin so that $E^{*} \approx E_{\text {skin }} /\left(1-\nu_{\text {skin }}^{2}\right)$. The elastic behaviour of the skin depends on the length scale of the contact as reported by Van Kuilenburg et al. [8]. It was shown for a spherical probe in contact with the skin that the elastic behaviour can be described as a function of the length scale of the contact by a closed-form expression by using the concept of an effective elastic modulus. Due to the large variation in finger properties, consistent data sets are not available. Given the layered structure of the skin, which is supported by the stiffer bone of the distal phalanx, for the fingerpad the same mechanical behaviour can be expected. When considering different size levels, for a spherical contact the evolution of the elastic modulus can be described as a function of the contact radius by a power law. At the macro scale, i.e. the fingertip, which is characterized by a contact radius in the order of millimeters, the elastic modulus increases with increasing contact radius due to 
the increasing contribution to the effective elastic modulus of the much stiffer bone. At the scale of surface roughness, at contact radii of several $\mu \mathrm{m}$, the elastic modulus is determined mainly by the stratum corneum, thus being almost independent of the length scale $a$. In between, the meso scale is the level of the fingerprint ridges.

At the macro scale the elastic modulus of the fingerpad varies with the contact radius following

$$
E_{0}^{*}=C_{0} a_{0}^{\alpha}
$$

Assuming that when normal loads are small the fingertip behaves as an elastic sphere, the nominal contact area of the fingerpad can be obtained from Eqs. (4) and (17) by

$$
A_{0}=\pi K_{0}^{2} F^{\frac{2}{3+\alpha}}
$$

where

$$
K_{0}=\left(\frac{3 R_{0}}{4 C_{0}}\right)^{\frac{1}{3+\alpha}}
$$

\subsection{Fingerprint ridges}

Although in most mechanical components the contact between two rough surfaces can be described by the contact between a flat and a surface having a so-called equivalent roughness, for skin contacts this assumption does not hold. In the contact between the skin and, in most cases, the harder product surface length scales and mechanical properties of the surface features are considerably different. Therefore, the contact areas formed by the fingerprint ridges and the topography of the countersurface are calculated in subsequent steps.

Surface topography measurements show that the shape of the fingerprint ridges is approximately trapezoidal. Although different ridge patterns (such as arch, loop, symmetrical and spiral) exist, to a first approximation the contacting areas between the fingerprint ridges and the countersurface are calculated by describing these contacts as annulus shaped contacts. Assuming that the contact area is determined by the number of ridges that are in contact rather than deformation of the ridges [9], the contact area can be determined from the ridge density $\eta$ and ridge width $2 a_{1}$. From the number of fingerprint ridges in contact with the skin $\eta a_{0}$ the total length of the contacts is calculated as

$$
l=\pi \eta a_{0}^{2}
$$


Paper C

which gives for the apparent contact area of the fingerprint ridges

$$
A_{1}=2 \pi \eta a_{1} K_{0}^{2} F^{\frac{2}{3+\alpha}}
$$

\subsection{Regular surface textures}

The contact between the asperities and the fingerprint ridges is formed by the stratum corneum, the top layer of the skin. Whereas the thickness of the stratum corneum is 10-20 $\mu \mathrm{m}$ for hairy skin, for glabrous skin it is much thicker. Values between 210 and $640 \mu \mathrm{m}$ were measured at the fingerpad by Liu et al. [21]. The composition and hydration level of the stratum corneum varies with depth. Hence the mechanical properties change; the elastic modulus decreasing with increasing indentation depth as shown by Yuan and Verma [6]. They measured the elastic modulus of isolated pig stratum corneum in an indentation test at depths ranging from about 0.15 to $1.2 \mu \mathrm{m}$. The elastic modulus was found to decrease with indentation depth and, when plotted against the contact radius, follows a power law $E \approx C a^{\gamma}$ with exponent $\gamma \approx-0.79$. The same trend was observed by Pailler-Mattéi et al. [7] who measured elastic moduli at depths ranging from 0.01 to $5 \mu \mathrm{m}$. An exponent $\gamma \approx$ -0.77 can be calculated from their results. In the contact region of a single asperity the elastic modulus of the stratum corneum of the fingerpad is assumed to follow a power law analogous to Eq. (17). Because of the high thickness, the role of the lower skin layers in the mechanical behaviour of the fingerpad stratum corneum is considered negligible at this length scale.

Since untreated human skin is covered with a lipid film, a mixture of water and sebum, when studying the contact behaviour of the skin adhesive forces cannot be neglected. Pailler-Mattéi and Zahouani [44] used Eq. 9 to calculate the work of adhesion from indentation experiments on in vivo skin. Experiments were carried out on normal, i.e. untreated, and dry, i.e. treated with ether, skin of the inner forearm. Results showed that a thin surface film on the skin is responsible for the adhesive behaviour. A dependence of the measured adhesive force on indentation velocity confirmed this conclusion. They found that JKR theory can only be used to predict adhesion force and contact geometry when the contact between the indenter and the skin approaches a sphere-on-flat geometry. So only at low normal loads, when the strains are less than $20 \%$, is the theory applicable. For untreated skin they found that the work of adhesion varies between 16 and $28 \mathrm{~mJ} / \mathrm{m}^{2}$ for radii of curvature of 2.25 to $6.35 \mathrm{~mm}$ and normal loads between 20 and $80 \mathrm{mN}$. Water treatment increased the work of adhesion considerably $\left(W_{12}=107 \mathrm{~mJ} / \mathrm{m}^{2}\right)$, whereas it returned to normal values after the skin regained its normal condition. The sebum, sometimes referred to as skin grease, is produced by the sebaceous glands, which are associated with hairs [5]. Hence the glabrous skin of the fingerpad does not contain any sebaceous glands. However, 
the fingerpad has a high density of sweat ducts. Between 150 and 1000 sweat ducts per $\mathrm{cm}^{2}[10,21]$ supply the skin of the fingerpad with a small amount of sweat to enhance grip by increasing adhesion. The JKR model is used to account for normal adhesion.


Figure 6. Schematic overview of the contact dimensions of the contact formed by the asperities at the micro scale.

The number of asperities in contact with the skin of the fingerprint ridges is obtained analogous to Eq. (7) so that

$$
A_{1}=N \lambda^{2}
$$

The contact area between an individual asperity and the skin is obtained from

$$
A_{i}=\pi K_{i}^{2} \cdot\left(\frac{F_{a p p}}{N}\right)^{\frac{2}{3+\gamma}}
$$

where $K_{i}$ is defined analogous to Eq. 19 and $F_{a p p}$ is given by Eq. (11). The contact ratio and relative indentation for the contact between the fingerpad and a regular surface texture can now be described following

$$
\frac{A_{\text {real }}}{A_{0}}=\pi\left(2 a_{1} \eta\right)^{\frac{1+\gamma}{3+\gamma}}\left(\frac{3}{4 C_{i}}\right)^{\frac{2}{3+\gamma}} R^{\frac{2}{3+\gamma}} \lambda^{\frac{-2(1+\gamma)}{3+\gamma}}\left(\frac{F_{a p p}}{A_{0}}\right)^{\frac{2}{3+\gamma}}
$$


Table 3. Parameter values used in calculations

\begin{tabular}{ll}
\hline Parameter & Value \\
\hline Elastic modulus steel $E_{1}$ & $210 \mathrm{GPa}$ \\
Poisson ratio steel $\nu_{1}$ & 0.3 \\
Fingerpad radius of curvature $R_{0}$ & $11 \mathrm{~mm}$ \\
Applied normal load $F$ & $1,2 \mathrm{~N}$ \\
Work of adhesion $W_{12}$ & $20 \mathrm{mN} / \mathrm{m}$ \\
Fingerprint ridge density $\eta$ & $1.680 / \mathrm{mm}$ \\
Half-width fingerprint ridge $a_{1}$ & $175 \mu \mathrm{m}$ \\
Constant $C_{i}$ & 5000 \\
Index $\gamma$ & -0.7 \\
\hline
\end{tabular}

$$
\frac{a}{R}=\left(2 a_{1} \eta\right)^{\frac{-1}{3+\gamma}}\left(\frac{3}{4 C_{i}}\right)^{\frac{1}{3+\gamma}} R^{\frac{-(2+\gamma)}{3+\gamma}} \lambda^{\frac{2}{3+\gamma}}\left(\frac{F_{a p p}}{A_{0}}\right)^{\frac{1}{3+\gamma}}
$$

\section{Results and discussion}
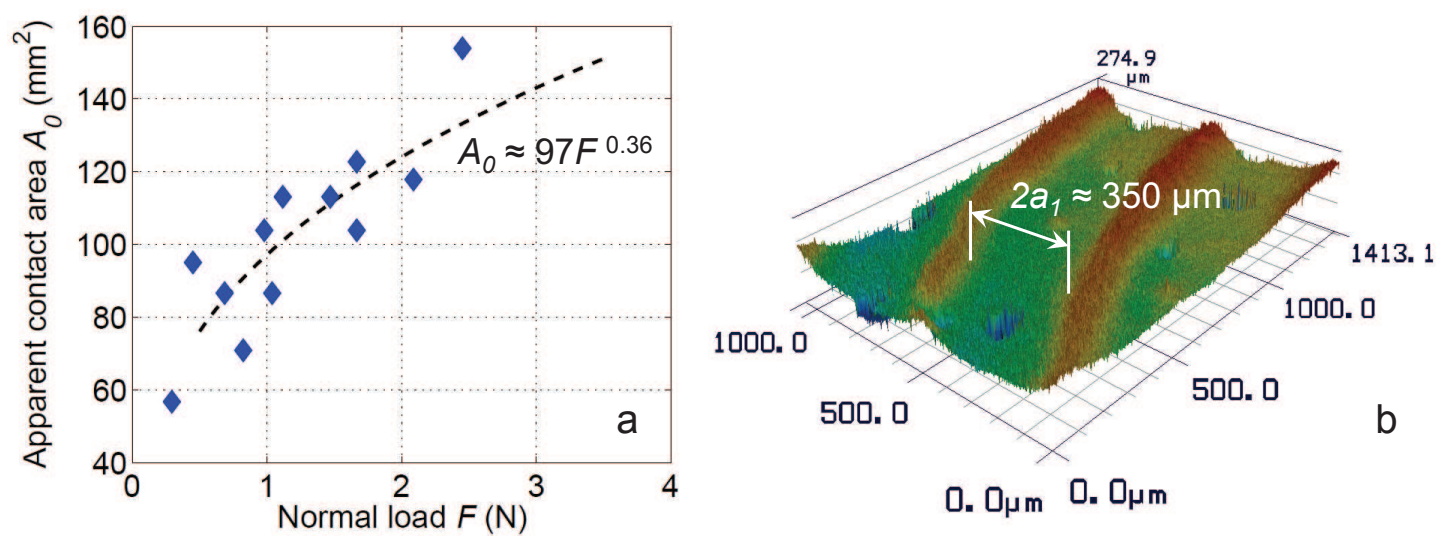

Figure 7. Apparent contact area $A_{0}$ of the fingerpad measured as a function of normal load $F$ (a) and width and topography of a (replica of a) fingerprint ridge (b).

Geometrical parameters were measured from the left (dominant) index finger of a 37year-old male subject. The radius of curvature of the undeformed finger was measured in two directions by fitting the fingertip in a circle template which is normally used in technical drawing. The reduced radius of curvature $R_{0} \approx 11 \mathrm{~mm}$. The relation between 
the apparent contact area and normal load was measured by pressing the inked finger on a piece of paper supported by a digitial kitchen scale, after which the outer dimensions of the fingerprints were measured. The orientation angle of the finger was approximately $30^{\circ}$. Figure 7 a plots the apparent contact area $A_{0}$ as a function of normal load $F$. A power law fitted through the results yields an exponent $n=0.36$. Using Eq. (18) this gives $\alpha=2.56$, showing that at this scale the effective elastic modulus increases strongly with the contact length scale. From Eq. (19) the effective elastic modulus can be calculated, which at 1 $\mathrm{N}$ normal load, is approximately $47 \mathrm{kPa}$, which is comparable to values obtained by e.g. Childs et al. [19].

The topography of the skin of the fingerpad, the fingerprint ridges, was obtained from a replica made using a synthetic rubber replica compound (Compound 101RF, Microset Products Ltd, UK) as shown in Fig. 7b. The surface geometry was measured using a 3D Laser Confocal Microscope model VK-9700 (Keyence, USA). The width of the fingerprint ridges varies between 300 and $400 \mu \mathrm{m}$, whereas the width of the furrows is $210-280 \mu \mathrm{m}$. The average density of the fingerprint ridges obtained from these values $\eta \approx 1.680 / \mathrm{mm}$. The amplitude of the surface features is 60-80 $\mu \mathrm{m}$.

The results from in vivo experiments are shown in Figs. 8-10. The standard deviation of the measurements is shown by error bars. For the textured surfaces a significantly lower friction was measured than for the smooth polished reference sample. The coefficient of friction measured against a smooth metal surface was 0.87 with a standard deviation $\sigma=$ 0.23. Stick-slip effects were clearly noticed during these measurements causing a large variation within and between measurements. Experiments against the textured surfaces showed smooth friction signals and standard deviations lower than 0.05. This variation is rather low when compared to the variation usually observed in in vivo skin friction measurements. To exclude the effect of factors such as the hydration level of the skin measurements were carried out in a random order. In between measurements the samples and the skin were cleaned to assure the surface condition of both sample and skin being the same in all measurements. Pasumarty et al. [45] investigated the role of sweat secretion and contact occlusion in the tribology of the human fingerpad. They found that occlusion might lead to very high coefficients of friction, especially when sliding against smooth surfaces. However, in this study occlusion time is very short, so occlusion is not expected to play a role in the observed friction behaviour. Sweat secretion brings the skin in a damp state, which is characterized by a higher moisture level than when the skin is in the dry state. During the experimental program the hydration level of the test area measured using the Corneometer CM 825 was $64 \pm 4$ AU, so that the skin of the fingerpad can be considered slightly hydrated. The variation in the hydration level is considered to be too small to have a significant influence on the observed friction behaviour [39-41]. 


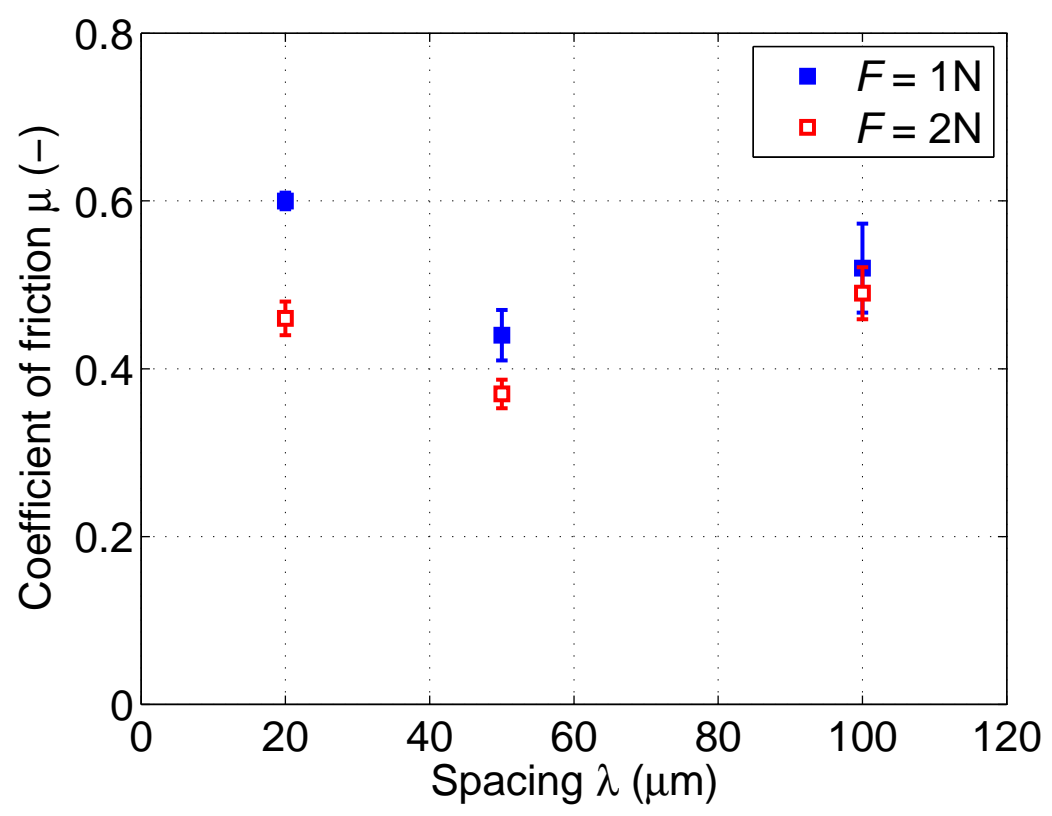

Figure 8. Measured coefficient of friction of the fingerpad (average of 3 measurements) as a function of tip spacing $\lambda$ and normal load $F$ for tip radius $R=5 \mu \mathrm{m}$.



Figure 9. Measured coefficient of friction of the fingerpad (average of 3 measurements) as a function of tip radius $R$ for different spacing $\lambda$ and normal load $F$. 


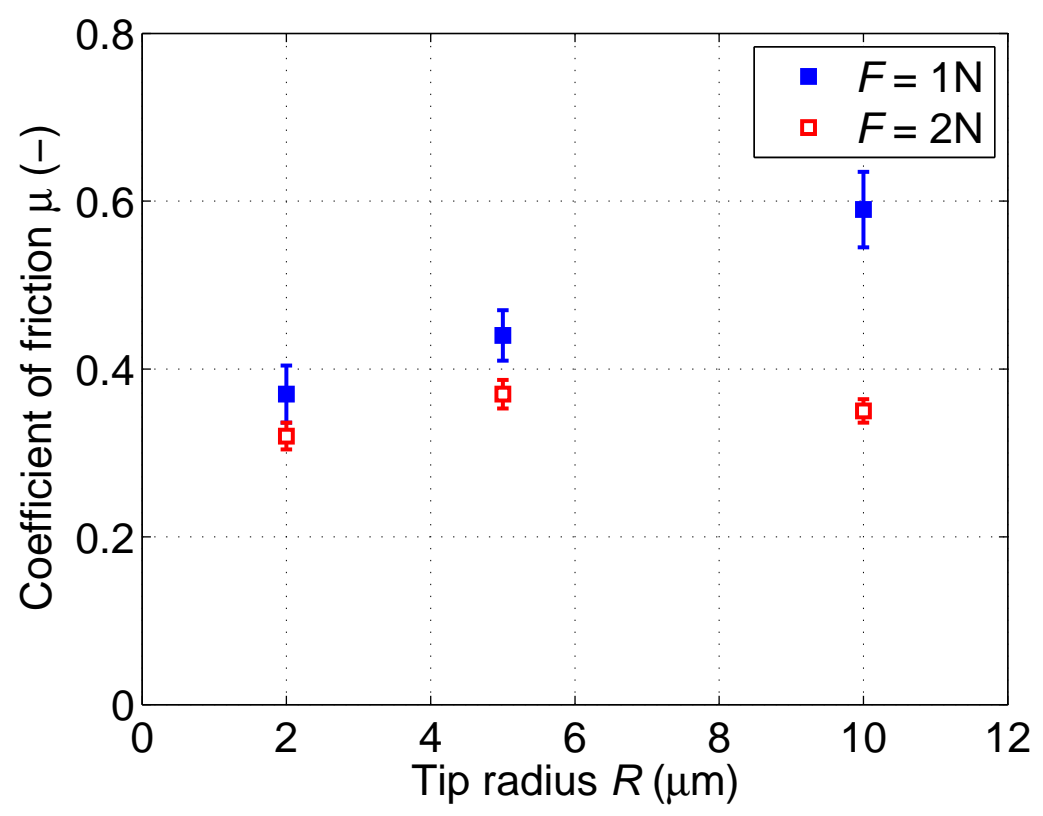

Figure 10. Measured coefficient of friction of the fingerpad (average of 3 measurements) as a function of tip radius $R$ at $\lambda / R=10$ for different normal load $F$.

Figure 8 shows the coefficient of friction as a function of tip spacing $\lambda$. For constant tip radius $R=5 \mu \mathrm{m}$ the coefficient of friction decreases with increasing spacing to a minimum after which an increase of the friction is observed. The measured coefficient of friction is lower for a higher applied normal load, which can be explained from the adhesion term in the two-term friction model. The contribution of normal adhesion between the two surfaces decreases with increasing applied normal load. This effect is more pronounced at small tip spacing, or high asperity density.

Figure 9 shows the coefficient of friction as a function of asperity tip radius. At $1 \mathrm{~N}$ normal load the coefficient of friction shows an increase with increasing tip radius $R$, which can be explained from the increasing contribution of normal adhesion, being proportional to the tip radius. However, at $2 \mathrm{~N}$ normal load and $100 \mu \mathrm{m}$ tip spacing the coefficient of friction appears to decrease with tip radius. With increasing normal load the relative contribution of the normal adhesion decreases. Furthermore, calculations show that with increasing tip spacing, the real contact area decreases, whereas the relative indentation increases as shown in Fig. 11. The relative indentation decreases with increasing tip radius.

For a constant value of $\lambda / R=10$ the coefficient of friction increases with increasing tip radius $R$ as shown in Fig. 10, except for measurement with spacing $100 \mu \mathrm{m}$ at normal 

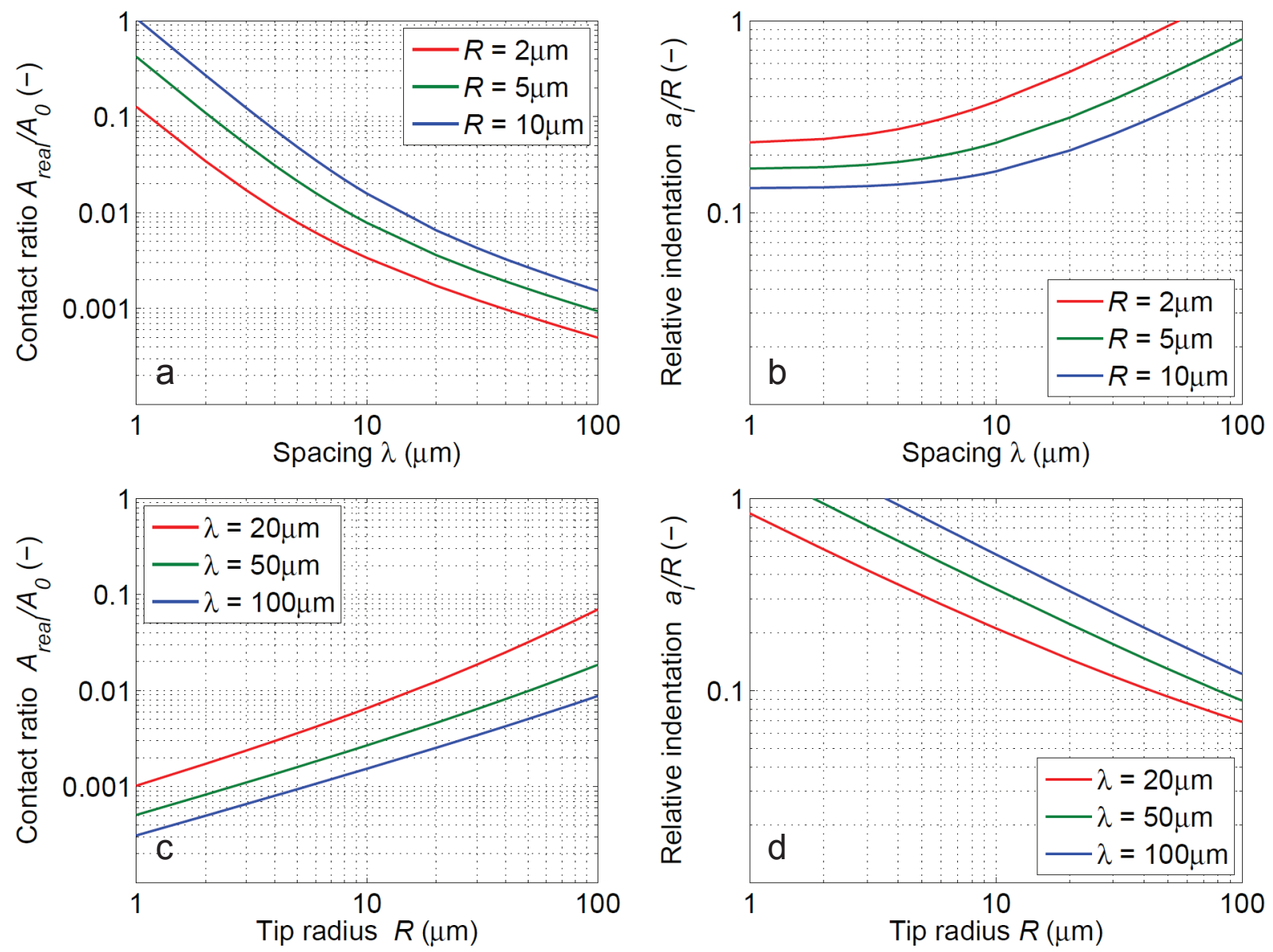

Figure 11. Contact ratio $A_{\text {real }} / A_{0}$ and relative indentation $a_{i} / R$ calculated as a function of spacing $\lambda$ for different tip radii $R(\mathrm{a}, \mathrm{b})$ and as a function of tip radius $R$ for different spacing $\lambda(\mathrm{c}, \mathrm{d})$ at $F=1 \mathrm{~N}$.

load 2 N. Figures 8-10 show that the measured coefficient of friction is lower for a higher applied normal load.

Figure 11 shows the calculated contact ratio and relative indentation. Considering the fact that adhesive shear is the dominant friction mechanism, the observed friction behaviour can be explained from the contact model, which shows that the real contact area increases with asperity tip radius and decreases with tip spacing. Furthermore, the contribution of normal adhesion to the effective normal load on the asperities increases with increasing tip radius, thus increasing the coefficient of friction. This effect becomes less pronounced for larger spacing. At a constant normal load the force acting on each asperity increases with increasing spacing so that the relative contribution of the normal adhesion to the effective normal load decreases. Since the indentation depth increases with 
spacing, after reaching a minimum the coefficient of friction will increase with spacing due to the growing contribution of deformation to the total friction force.

When the spacing between the asperity tips becomes smaller than the tip radius the surface texture approaches the limit case of a flat, smooth surface. With the spacing increasing to large lengths, deformation of the skin becomes so large, that contact between the skin and the lower surface occurs, thus defining another limit case. This actually was the case with the $200 \mu \mathrm{m}$ spacing sample, which was therefore not considered any further in this study. The validity of the contact model is limited by the contact ratio $A_{\text {real }} / A_{0}<1$ and the relative indentation $a / R \ll 1$.

The relationship between surface roughness and friction behaviour within the scope of skin friction was discussed earlier by Masen [25]. It was stated that for many engineering surfaces the product of asperity density, tip radius and standard deviation of the asperity height distribution is constant. The asperity density does not change much with changing roughness, from which it was concluded that asperity radius $R$ and roughness $R_{a}$ are inversely proportional. Several authors [10, 22-25] report an increase of the measured coefficient of friction with decreasing roughness amplitude, which might thus be attributed to the increase in asperity radius. This theory is confirmed by the results obtained using textured surfaces in this study.

From the friction results depicted in Figs. 8 and 9 the viscoelastic loss modulus $\beta$ and surface shear strength $\tau$ can be approximated using Eqs. (24) and (25). Yuan and Verma measured the viscoelastic loss modulus $\beta$ from dynamic indentation experiments finding a value of 0.31 for dry and 0.79 for wet skin [6]. Since the skin of the fingerpad is slightly hydrated as shown earlier, a value for $\beta=0.55$ was used in the calculations. A surface shear strength varying between 1.4 and $4.5 \mathrm{MPa}$ is calculated from the measured friction coefficients, which is comparable to values reported by e.g. Persson et al. [46]. The calculated shear strength does not change considerably as a function of $\beta$. A closer examination of the deformation component of friction shows that the coefficient of deformation friction varies between 0.03 and 0.08 for $\beta=0.5$ and between 0.06 and 0.15 for $\beta=1$.

\section{Conclusions}

The friction behaviour of the human fingerpad as a function of asperity geometry was investigated experimentally. Surface textures consisting of evenly distributed sphericallytipped asperities were produced using ultra-short pulsed laser technology to enable in vivo testing. Using analytical expressions available from contact mechanics theory, a multiscale model was developed to explain the observed friction behaviour as a function of texture geometry, normal load and skin mechanical properties. 
Since the skin is a rather complex material, its properties changing between persons, with anatomical site and with the environment, the friction behaviour of textured surfaces was first investigated in an idealized model system. Ball-on-disk experiments using a polypropylene ball enabled the characterization of the friction mechanisms occurring between a rigid textured surface sliding against a softer compliant material, thus confirming the observations from in vivo experiments on the fingerpad.

The observations of in vivo measurements can be explained using a relatively simple analytical model and show that the coefficient of friction increases with increasing tip radius. This effect can be attributed to the normal adhesion force acting on each asperity, which is proportional to the tip radius. The coefficient of friction increases with increasing asperity density. With the number of asperities in contact increasing, the contribution of the normal adhesion leads to an increase of the measured coefficient of friction.

A minimum value for the coefficient of friction seems to exist as a function of asperity density. When the number of asperities in contact decreases, the increase of the indentation depth leads to an increase of the deformation component of friction. A maximum value for tip spacing exists above which the contact is not determined by the texture properties only. This maximum tip spacing is related to the width of the fingerprint ridges.

The friction force is approximately proportional to the contact load. Due to the contribution of normal adhesion forces, which are dependent on surface properties only, increasing the externally applied normal load leads to a decrease in the measured coefficient of friction.

This study showed that complex behaviour of the fingerpad sliding against a textured surface can be explained using relatively simple analytical expressions, thus increasing our understanding. Furthermore, the developed model can be used to estimate the friction behaviour as a function of asperity geometry and operational conditions. The use of simple expressions enables the translation to design guidelines for the engineering of surfaces with prescribed friction behaviour.

\section{Acknowledgements}

This research was carried out under project number M63.2.10409 in the framework of the Research Program of the Materials innovation institute M2i (www.m2i.nl).

\section{References}

1. L.J. Wolfram. Friction of skin. Journal of the Society of Cosmetic Chemists, 34:465476, 1983. 
2. S. A. Johnson, D. M. Gorman, M. J. Adams, and B. J. Briscoe. The friction and lubrication of human stratum corneum. In C.M. Taylor D. Dowson, editor, Thin Films in Tribology, Proceedings of the 19th Leeds-Lyon Symposium on Tribology held at the Institute of Tribology, University of Leeds, volume 25, pages 663-672. Elsevier, 1993.

3. M. J. Adams, B. J. Briscoe, and S. A. Johnson. Friction and lubrication of human skin. Tribology Letters, 26(3):239-253, 2007.

4. J. A. Greenwood and J. B. P. Williamson. Contact of nominally flat surfaces. Proceedings of the Royal Society of London. Series A, Mathematical and Physical Sciences, 295(1442):300-319, 1966.

5. Desmond J. Tobin. Biochemistry of human skin - our brain on the outside. Chemical Society Reviews, 35(1):52-67, 2006.

6. Yonghui Yuan and Ritu Verma. Measuring microelastic properties of stratum corneum. Colloids and Surfaces B: Biointerfaces, 48(1):6-12, 2006.

7. C. Pailler-Mattéi, S. Pavan, R. Vargiolu, F. Pirot, F. Falson, and H. Zahouani. Contribution of stratum corneum in determining bio-tribological properties of the human skin. Wear, 263(7-12):1038-1043, 2007.

8. J. Van Kuilenburg, M. A. Masen, and E. Van der Heide. Contact modelling of human skin: What value to use for the modulus of elasticity? Proceedings of the Institution of Mechanical Engineers, Part J: Journal of Engineering Tribology.

9. R. T. Spurr. Fingertip friction. Wear, 39(1):167-171, 1976.

10. O. S. Dinç, C. M. Ettles, S. J. Calabrese, and H. A. Scarton. Some parameters affecting tactile friction. Journal of Tribology, 113(3):512-517, 1991.

11. S. Derler and L.-C. Gerhardt. Tribology of skin: Review and analysis of experimental results for the friction coefficient of human skin. Tribology Letters, 45:1-27, 2011.

12. H. Y. Han, A. Shimada, and S. Kawamura. Analysis of friction on human fingers and design of artificial fingers. In Proceedings of the 1996 IEEE International Conference on Robotics and Automation, volume 4, pages 3061-3066. IEEE, 1996.

13. P. H. Warman and A. R. Ennos. Fingerprints are unlikely to increase the friction of primate fingerpads. Journal of Experimental Biology, 212(13):2016-2022, 2009. 
14. Thibaut André, Philippe Lefévre, and Jean-Louis Thonnard. A continuous measure of fingertip friction during precision grip. Journal of Neuroscience Methods, 179(2):224229, 2009.

15. Makoto Tomimoto. The frictional pattern of tactile sensations in anthropomorphic fingertip. Tribology International, 44(11):1340-1347, 2011.

16. T. Maeno, K. Kobayashi, and N. Yamazaki. Relationship between the structure of human finger tissue and the location of tactile receptors. JSME International Journal Series C, 41:94-100, 1998.

17. Toshiharu Soneda and Ken Nakano. Investigation of the vibration detectability of human fingers supported by the observation of contact zones. In Proceedings of the 2nd International Conference on Advanced Tribology, Singapore, 2008, paper no. iCAT288, 2008 .

18. S. Derler, L.-C. Gerhardt, A. Lenz, E. Bertaux, and M. Hadad. Friction of human skin against smooth and rough glass as a function of the contact pressure. Tribology International, 42(11-12):1565-1574, 2009.

19. T H C Childs and B Henson. Human tactile perception of screen-printed surfaces: Selfreport and contact mechanics experiments. Proceedings of the Institution of Mechanical Engineers, Part J: Journal of Engineering Tribology, 221(3):427-441, 2007.

20. S. E. Tomlinson. Understanding the friction between human fingers and contacting surfaces. PhD thesis, The University of Sheffield, 2009.

21. X. Liu, Z. Lu, R. Lewis, M.J. Carr, and S.J. Matcher. Feasibility of using optical coherence tomography to study the influence of skin structure on finger friction. Tribology International, (0).

22. T. Sasada. The friction of human skin. In Proceedings of the 21st Biotribology Symposium, JSME, Fukuoka, 2000, pages 46-52, 2000.

23. M. G. Gee, P. Tomlins, A. Calver, R. H. Darling, and M. Rides. A new friction measurement system for the frictional component of touch. Wear, 259(712):1437-1442, 2005.

24. X Liu, Z Yue, Z Cai, D G Chetwynd, and S T Smith. Quantifying touch-feel perception: tribological aspects. Measurement Science and Technology, 19(8):084007, 2008. 
25. M. A. Masen. A systems based experimental approach to tactile friction. Journal of the Mechanical Behavior of Biomedical Materials, 4:1620-1626, 2011.

26. S. E. Tomlinson, R. Lewis, and M. J. Carré. The effect of normal force and roughness on friction in human finger contact. Wear, 267(58):1311-1318, 2009.

27. Horst Czichos, editor. Tribology: A systems approach to the science and technology of friction, lubrication and wear. Elsevier, 1978.

28. Khaled Elleuch, Riadh Elleuch, and Hassan Zahouani. Comparison of elastic and tactile behavior of human skin and elastomeric materials through tribological tests. Polymer Engineering \& Science, 46(12):1715-1720, 2006.

29. S. Derler, U. Schrade, and L.-C. Gerhardt. Tribology of human skin and mechanical skin equivalents in contact with textiles. Wear, 263(7-12):1112-1116, 2007.

30. E. Van der Heide, C. M. Lossie, K. J. C. Van Bommel, S. A. F. Reinders, and H. B. M. Lenting. Experimental investigation of a polymer coating in sliding contact with skin-equivalent silicone rubber in an aqueous environment. Tribology Transactions, $53(6): 842,2010$.

31. C. Guerra and C. J. Schwartz. Development of a synthetic skin simulant platform for the investigation of dermal blistering mechanics. Tribology Letters, August 2011.

32. J A Greenwood and D Tabor. The friction of hard sliders on lubricated rubber: The importance of deformation losses. Proceedings of the Physical Society, 71(6):989-1001, 1958.

33. H. Hertz. Über die Berührung fester elastischer Körper. Journal für die reine und angewandte Mathematik, 92:156-171, 1882.

34. K. L. Johnson. Contact mechanics. Cambridge University Press, Cambridge, 1999.

35. Edward Dintwa, Engelbert Tijskens, and Herman Ramon. On the accuracy of the Hertz model to describe the normal contact of soft elastic spheres. Granular Matter, 10:209-221, 2007.

36. K. L. Johnson and J. A. Greenwood. An adhesion map for the contact of elastic spheres. Journal of Colloid and Interface Science, 192(2):326-333, 1997.

37. K. L. Johnson, K. Kendall, and A. D. Roberts. Surface energy and the contact of elastic solids. Proceedings of the Royal Society of London. A. Mathematical and Physical Sciences, 324(1558):301 -313, 1971. 
38. J S̆kvarla. Hydrophobic interaction between macroscopic and microscopic surfaces. unification using surface thermodynamics. Advances in Colloid and Interface Science, 91(3):335-390, 2001.

39. Anastasia B Cua, K. P. Wilhelm, and H. I. Maibach. Frictional properties of human skin: relation to age, sex and anatomical region, stratum corneum hydration and transepidermal water loss. British Journal of Dermatology, 123(4):473-479, 1990.

40. L.-C Gerhardt, V Strässle, A Lenz, N. D. Spencer, and S Derler. Influence of epidermal hydration on the friction of human skin against textiles. Journal of The Royal Society Interface, 5(28):1317 -1328, 2008.

41. M. Kwiatkowska, S. E. Franklin, C. P. Hendriks, and K. Kwiatkowski. Friction and deformation behaviour of human skin. Wear, 267(5-8):1264-1273, 2009.

42. S. E. Tomlinson, M. J. Carré, R. Lewis, and S. E. Franklin. Human finger contact with small, triangular ridged surfaces. Wear, 271(9-10):2346-2353, 2011.

43. F. P. Bowden, A. J. W. Moore, and D. Tabor. The ploughing and adhesion of sliding metals. Journal of Applied Physics, 14(2):80-91, 1943.

44. C. Pailler-Mattéi and H. Zahouani. Analysis of adhesive behaviour of human skin in vivo by an indentation test. Tribology International, 39(1):12-21, 2006.

45. Subrahmanyam Pasumarty, Simon Johnson, Simon Watson, and Michael Adams. Friction of the human finger pad: Influence of moisture, occlusion and velocity. Tribology Letters, pages 1-21, 2011.

46. B. N. J. Persson, A. Kovalev, and S. N. Gorb. Contact mechanics and friction on dry and wet human skin. Tribology Letters. 



\section{Paper D}

An experimental study on the relation between surface texture and tactile friction

J. van Kuilenburg, M.A. Masen, M.N.W. Groenendijk, V. Bana and E. van der Heide

Tribology International, Vol. 48, 2012, pp. 15-21

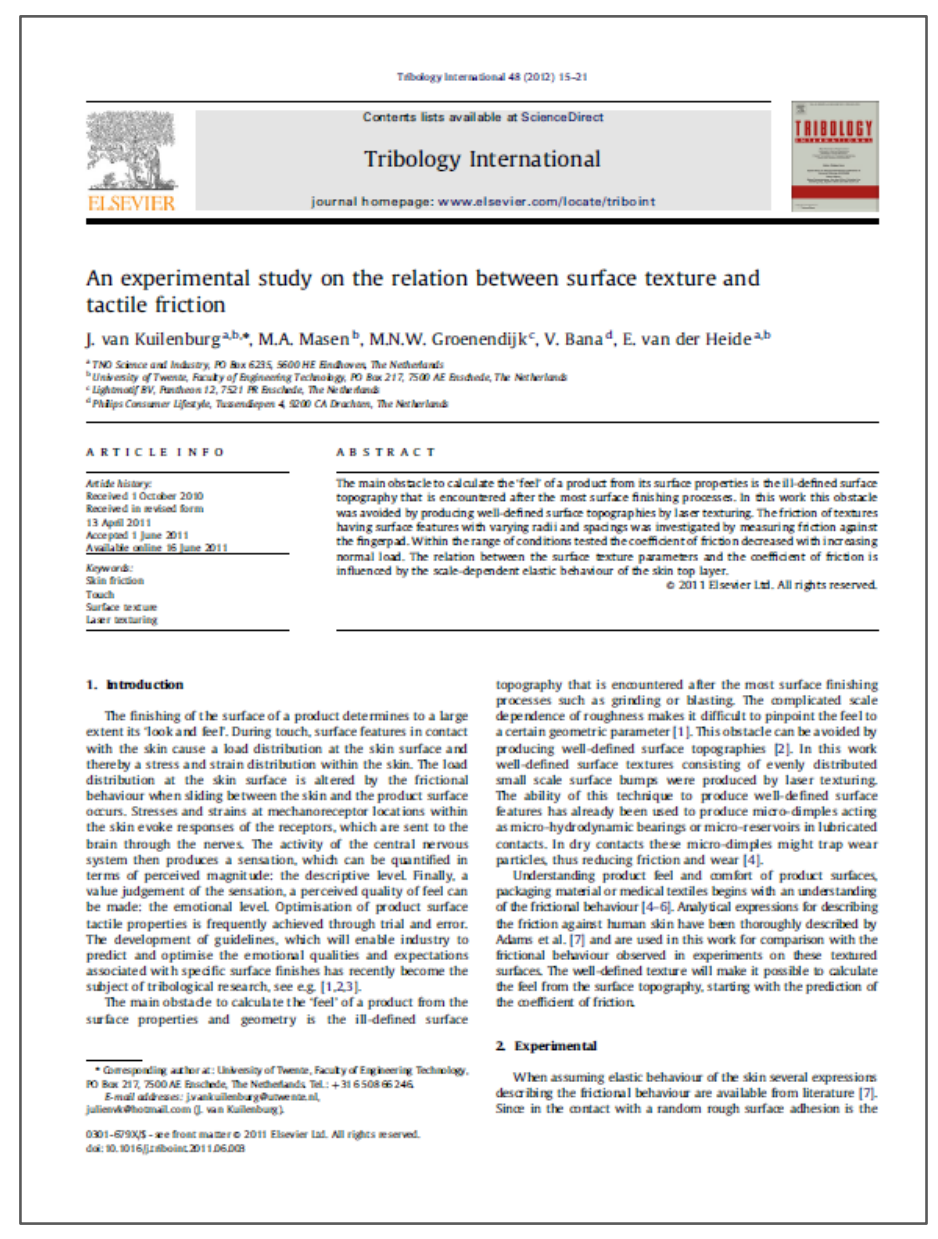





\title{
An experimental study on the relation between surface texture and tactile friction
}

\author{
J. van Kuilenburg, M.A. Masen, M.N.W. Groenendijk, V. Bana and E. van der Heide
}

\begin{abstract}
The main obstacle to calculating the 'feel' of a product from its surface properties is the illdefined surface topography that is encountered after most surface finishing processes. In this work, this obstacle was avoided by producing well-defined surface topographies by laser texturing. The friction of textures that have surface features with varying radii and spacings was investigated by measuring friction against the fingerpad. Within the range of conditions tested, the coefficient of friction decreased with increasing normal load. The relation between the surface texture parameters and the coefficient of friction is influenced by the scaledependent elastic behaviour of the skin top layer.
\end{abstract}

\section{Keywords}

skin friction; touch; surface texture; laser texturing

\section{Introduction}

The finishing of the surface of a product determines to a large extent its 'look and feel'. During touch, surface features in contact with the skin cause a load distribution at the skin surface and thereby a stress and strain distribution within the skin. The load distribution at the skin surface is altered by the frictional behaviour when sliding between the skin and the product surface occurs. Stresses and strains at mechanoreceptor locations within the skin evoke responses of the receptors, which are sent to the brain through the nerves. The activity of the central nervous system then produces a sensation which can be quantified in terms of perceived magnitude: the descriptive level. Finally, a value judgement of the sensation, a perceived quality of feel can be made: the emotional level. Optimization of product surface tactile properties is frequently achieved through trial and error. The development of guidelines, which will enable industry to predict and optimize the emotional qualities and expectations associated with specific surface finishes, has recently become the subject of tribological research, see e.g. [1, 2, 3].

The main obstacle to calculating the 'feel' of a product from the surface properties and geometry is the ill-defined surface topography that is encountered after most surface finishing processes such as grinding or blasting. The complicated scale dependence of roughness makes it difficult to pinpoint the feel to a certain geometric parameter [1]. This obstacle can be avoided by producing well-defined surface topographies [2]. In this work, well-defined surface textures consisting of evenly distributed small-scale surface bumps were 
produced by laser texturing. The ability of this technique to produce well-defined surface features has already been used to produce micro-dimples acting as micro-hydrodynamic bearings or micro-reservoirs in lubricated contacts. In dry contacts these micro-dimples might trap wear particles, thus reducing friction and wear [4].

Understanding product feel and comfort of product surfaces, packaging material or medical textiles begins with an understanding of the frictional behaviour [4, 5, 6]. Analytical expressions for describing the friction against human skin have been thoroughly described by Adams et al. [7] and are used in this work for comparison with the frictional behaviour observed in experiments on these textured surfaces. The well-defined texture will make it possible to calculate the feel from the surface topography, starting with the prediction of the coefficient of friction.

\section{Experimental}

When assuming elastic behaviour of the skin, several expressions describing the frictional behaviour are available from the literature [7]. Since, in the contact with a random rough, surface adhesion is the dominant friction mechanism the coefficient of friction is determined by the magnitude of the real contact area. Following, for example, Greenwood and Williamson theory for the contact of random rough surfaces, the coefficient of friction can be expressed as a function of roughness amplitude and mean asperity radius. The roughness parameters amplitude and asperity radius are mean values taken from a set of measured amplitudes and radii. Furthermore, these roughness amplitudes and radii depend strongly on measuring equipment and method. By using regular surface textures these uncertainties are avoided and the coefficient of friction can be predicted with greater accuracy.

\subsection{Materials}

Four different surface structures were made with picosecond laser pulses. Metal samples were produced directly on stainless strip material using laser ablation [8]. Polymer samples were produced by applying the structures on mould inserts and reproduction in a thermoplastic polyurethane (TPU). The macro-geometry of the samples is a circular flat with a $30 \mathrm{~mm}$ diameter. Micro-geometrical parameters of the different surface textures are summarized in Table 1.

The surface texture is composed from bumps having a spherical tip with radius $R$, which are evenly distributed with a spacing $\lambda$ between the tips as depicted in Figure 1. Four different surface textures were composed from two different radii and two different spacings. Radii and spacings were chosen such that the minimum and maximum values of the radius over spacing $R / \lambda$ differ by one order of magnitude, while the tip radii are small. A small tip radius was expected to result in a low contact area and thus relatively low friction.

SEM images of the metal and TPU samples are shown in Figs. 2 and 3 respectively. Figure 2 is representative for all metal samples and the injection moulded textures with tip radii $R=5$ 


\begin{tabular}{|c|c|c|c|c|}
\hline No. & $\begin{array}{c}\text { Radius } \\
R(\mu \mathrm{m})\end{array}$ & $\begin{array}{c}\text { Spacing } \\
\lambda(\mu \mathrm{m})\end{array}$ & $\begin{array}{c}R / \lambda \\
(-)\end{array}$ & $\begin{array}{c}\text { Height } \\
h(\mu \mathrm{m})\end{array}$ \\
\hline 1 & 1 & 30 & 0.033 & 30 \\
\hline 2 & 1 & 60 & 0.017 & 30 \\
\hline 3 & 5 & 60 & 0.083 & 20 \\
\hline 4 & 5 & 30 & 0.167 & 20 \\
\hline
\end{tabular}

Table 1. Surface textures produced by picosecond laser pulses. All textures are applied on metal and TPU (denoted as $\mathrm{m} 1, \mathrm{~m} 2, \ldots$ and TPU1, TPU2,...).

$\mu \mathrm{m}$. The two-dimensional waved surface described by a spacing and tip radius approaches a bi-sinusoidal surface with its crests, valleys and saddle points. With the injected moulded textures with tip radii $R=1 \mu \mathrm{m}$ the saddle points are stretched, appearing as ridges surrounding relatively large square valleys with the crests as small-scale surface features superposed on this grated pattern as can be seen from Fig. 3. It needs no explanation that the mechanisms responsible for the frictional behaviour of such a grated texture might be completely different than those of the bi-sinusoidal texture of Fig. 2. A further analysis of the contact mechanisms for such grated textures is not considered relevant within the scope of this work.

The metal and TPU samples were tested against the fingerpad skin of the left (dominant) index finger of a 35-year-old male subject.

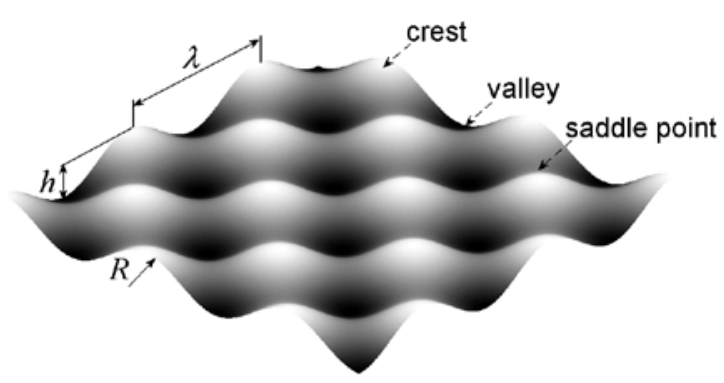

Figure 1. Definition of texture parameters of test samples produced by laser texturing. 


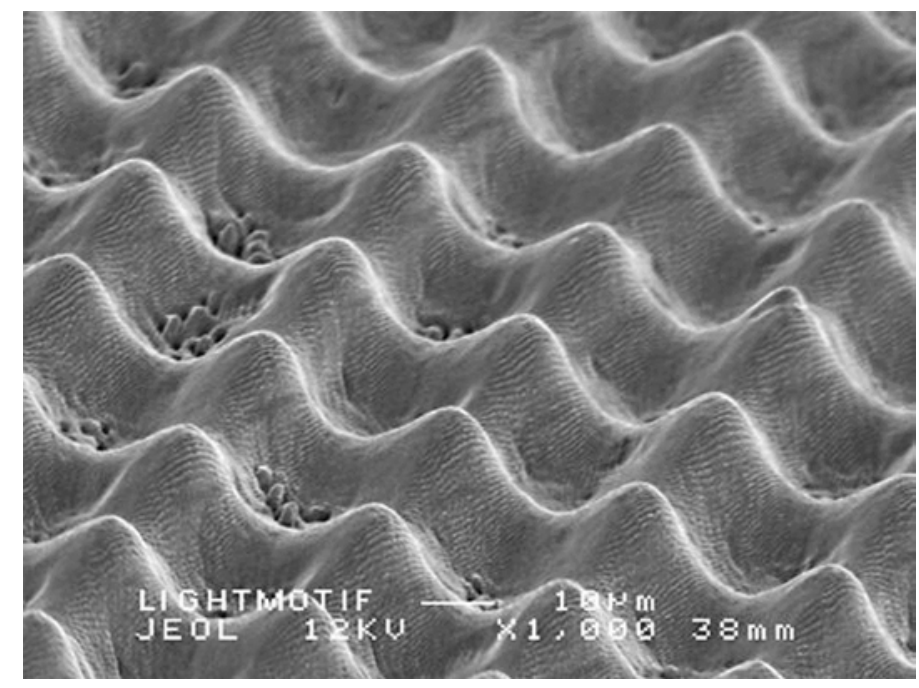

Figure 2. SEM image of metal sample (m4) with tip radius $R=5 \mu \mathrm{m}$ and spacing $\lambda=30 \mu \mathrm{m}$.

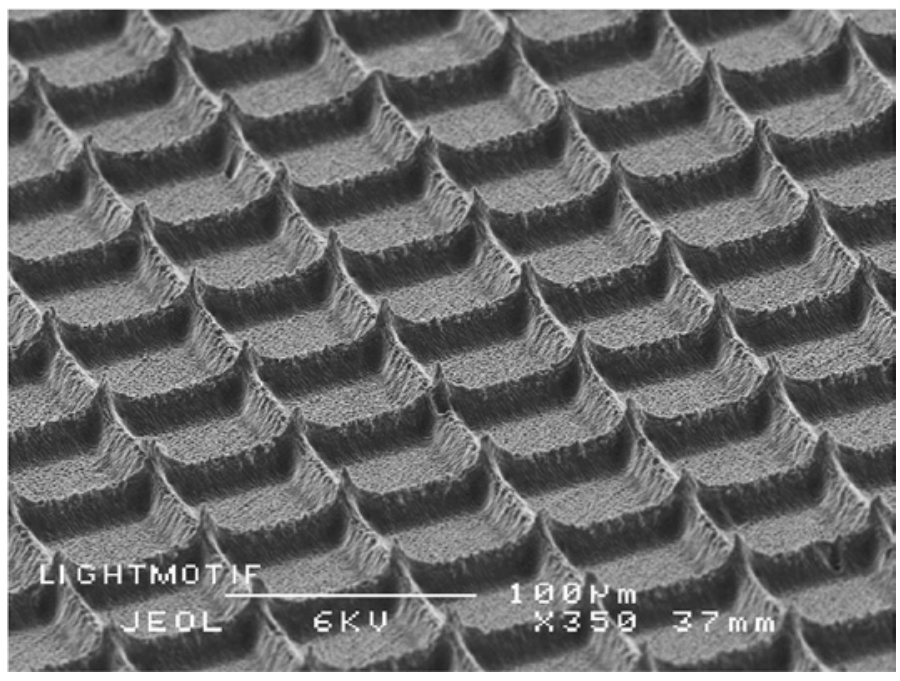

Figure 3. SEM image of TPU sample (TPU2) with tip radius $R=1 \mu \mathrm{m}$ and spacing $\lambda=60 \mu \mathrm{m}$.

\subsection{Methods}

Sliding friction was measured in vivo against human skin using the Skin Micro-Tribometer model UMT (CETR, USA) described by e.g. Sivamani et al. [9]. The tribometer was equipped with a 6-axis torque /force sensor (type TFH-6), which measures normal load in the range $0.5-60 \mathrm{~N}$ (7.5 $\mathrm{mN}$ resolution) and lateral (friction) loads in the range $0.2-20 \mathrm{~N}(2.5 \mathrm{mN}$ resolution). The normal loads used were $0.5,1$ and $2 \mathrm{~N}$. Stroke distance in fingerpad measurements was $12 \mathrm{~mm}$ at a sliding velocity of $12 \mathrm{~mm} / \mathrm{s}$. For the measurements of friction 
of the fingerpad the left index finger of the experimenter was positioned on a support under the sliding probe at an inclination of about $30^{\circ}$.

All tests were carried out at room temperature $20 \pm 2 \mathrm{C}^{\circ}$ and relative humidity $45 \pm 4 \%$. Each measurement consists of 3 cycles back and forth. The mean of the 6 strokes was taken as the mean coefficient of friction $\mu$.

During the test program the hydration level of the fingerpad skin surface was determined using a Corneometer ${ }^{\circledR}$ CM 825 (CK electronic GmbH, Germany). This device measures the hydration level of the upper 10-20 $\mu \mathrm{m}$ of the stratum corneum through capacitance. The hydration level is expressed in arbitrary units AU, <30 AU corresponding to dry skin, >50 AU corresponding to hydrated skin. The strong correlation between skin hydration level measured using the Corneometer ${ }^{\circledR}$ CM 825 and skin friction is illustrated by Gerhardt et al. [10].

\subsection{Sample preparation}

All samples were cleaned using ethanol before and in between the experiments. Before carrying out the experiments, the hands were washed using water and soap and airdried. After a rest period of approximately 10 minutes to allow the washed skin to regain its normal condition, the experiments on dry skin were carried out. Experiments on hydrated skin were carried out after submersion in water for at least 10 minutes. The fingerpad skin was hydrated by submersion, the skin of the forearm was hydrated by using a wet sponge. Verrillo et al. [11] showed that after submersion for 5 to 10 minutes hydration returns to normal levels between 5 to 15 minutes, much longer than the time needed for one experiment. Before the experiments excess water was removed using a paper tissue.

\section{Results and Discussion}

An overview of the coefficients of friction measured against dry skin is shown in Fig. 4. The mean coefficient of friction is given for the metal (m) and TPU samples for different normal loads. Smooth, non-textured samples were measured for reference and are noted m0 and TPU0. 


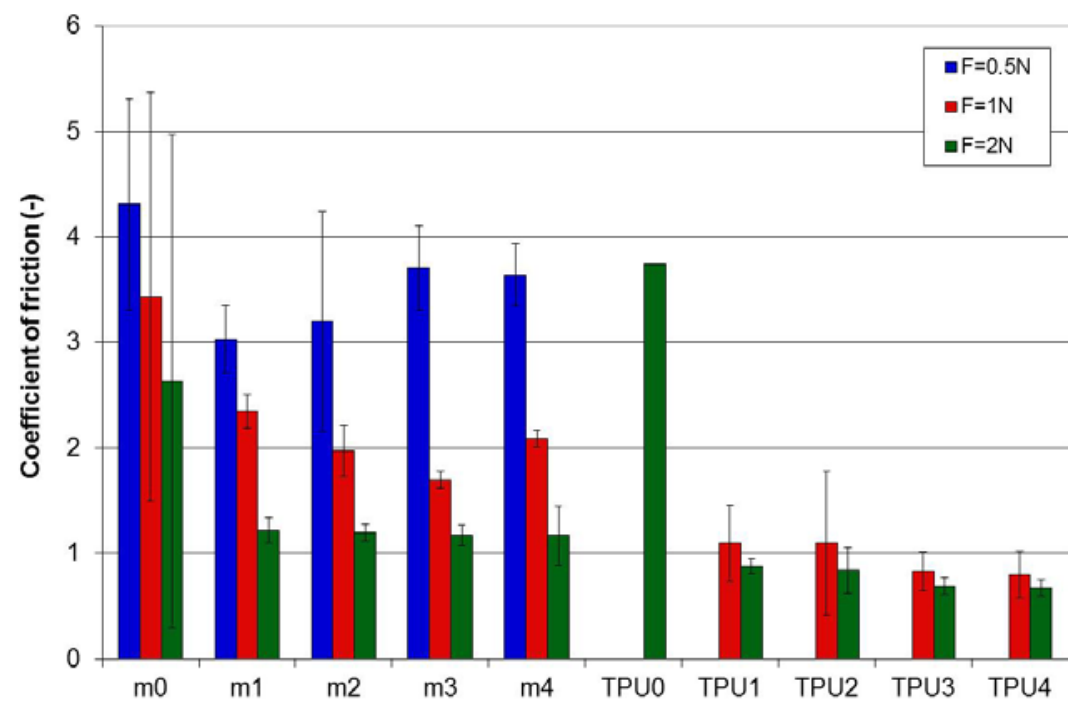

Figure 4. Overview of the coefficients of friction (mean $\pm 2 \bullet$ st.dev.) measured for metal (m) and TPU samples, $\mathrm{m} 0$ and TPU0 being smooth reference samples.

\subsection{General discussion}

The development of the contact area of a fingertip and a smooth surface was investigated by several authors $[2,12]$. Soneda and Nakano [12] measured the apparent and real contact areas of fingerpads as a function of contact load. For normal loads between 0.1 and $5 \mathrm{~N}$ they found the real contact area to increase with contact load following a power law with exponent 0.68 . They defined the real contact area as the area of the fingerprint ridges in contact with the smooth, flat surface and the apparent contact area as the area within the outline of the real contact area. In this work the apparent, or nominal, contact area $A_{0}$ is defined as the area of the fingerprint ridges in macroscopic contact with the countersurface. The real contact area $A_{r}$ is defined as the total area of the surface features which are in actual contact with the skin.

\subsubsection{Friction of regular surface textures}

The coefficient of friction is composed of a deformation and an adhesion component

$\mu=\mu_{\text {def }}+\mu_{\text {adh }}$

Expressions for the deformation and adhesion components of friction for a spherical indenter sliding against the skin are discussed by e.g. Adams et al. [7] and can be rewritten for the textures produced in this study. Since the surface features are arranged in a regular pattern having a pillar spacing $\lambda$, the number of surface features $N$ in contact with the skin can be approximated from the contact area $A_{0}$ using

$A_{0}=N \cdot \lambda^{2}$ 
The total normal load $F_{n}$ is composed of the normal loads on each surface feature $F_{n, i}$ following

$F_{n}=N \cdot F_{n, i}$

Assuming that the individual surface features of the textures are perfectly spherical and assuming elastic behaviour of the skin, the equations adopted from [6] can be rewritten by substituting Equations (2) and (3).

The coefficent of friction for deformation can now be expressed as a function of normal force $F_{n}$, real contact area $A_{0}$ and a surface texture parameter $R / \lambda$, the quotient of surface feature radius and spacing, following

$\mu_{\text {def }} \propto \beta \cdot E^{*-1 / 3} \cdot\left(\frac{R}{\lambda}\right)^{-2 / 3} \cdot\left(\frac{A_{0}}{F_{n}}\right)^{-1 / 3}$

where $E^{*}$ is the effective elastic modulus, in the case of a countersurface having a high elastic modulus determined mainly by the skin elastic modulus and $\beta$ is the skin viscoelastic loss fraction. This viscoelastic loss fraction can be estimated from the hysteresis loop, the area between the loading and unloading curve in an indentation experiment.

The coefficient of friction for adhesion can be expressed as

$\mu_{a d h} \propto \tau \cdot E^{*-2 / 3} \cdot\left(\frac{R}{\lambda}\right)^{2 / 3} \cdot\left(\frac{A_{0}}{F_{n}}\right)^{1 / 3}$

where $\tau$ is the shear stress of the skin-texture interface thus giving the friction force due to adhesion when being multiplied with the total area of the surface features in contact with the skin. The total coefficient of friction for the textures produced in this study can be expressed as a function of surface texture parameter $R / \lambda$ by substituting Equations (4) and (5) into Equation (1):

$\mu_{\text {text }} \propto C_{d e f} \cdot\left(\frac{R}{\lambda}\right)^{-2 / 3}+C_{a d h} \cdot\left(\frac{R}{\lambda}\right)^{2 / 3}$

Values for real contact area of the fingerpad as a function of normal load are measured by e.g. Childs et al. [2] and Soneda and Nakano [12]. At normal loads around $F_{n}=2 \mathrm{~N}$, the real contact area $A_{0}$ varies from 30 to $60 \mathrm{~mm}^{2}$. For $A_{0}$ a value of $60 \mathrm{~mm}^{2}$ is adopted. Mechanical properties from dry stratum corneum can be found in the literature $\beta \sim 0.31, E_{s c} \sim 100 \mathrm{MPa}$ [13] and $\tau \sim 10 \mathrm{MPa}$ [14]. Using these values the parameters $C_{d e f}$ and $C_{a d h}$ can be calculated. It is found that $C_{a d h} \sim 1.4$, thus being much larger than $C_{d e f} \sim 0.02$. From this it is expected that adhesion is the dominant mechanism within the range of texture parameters investigated in this work.

\subsubsection{Elastic behaviour of the contact zone}

The validity of equations (4) and (5) is based on the assumption of elastic behaviour of the contact zone. Furthermore, it is assumed that the real contact area is determined mainly through the properties of the stratum corneum, having a high stiffness, as opposed to the 
much more compliant epidermis. For this assumption to be valid, the contact radius of each individual surface feature, the Hertzian contact radius, $a$ should be smaller than the radius of this surface feature:

$a=\left(\frac{3 F_{n, i} \cdot R}{4 E^{*}}\right)^{1 / 3}<R$

Expressing the normal force on the contact in texture parameters $R$ and $\lambda$ using Equations (2) and (3) and setting the contact radius equal to the tip radius yields the following expression for the minimum elasticity for the above assumption to be valid:

$$
E^{*}>\frac{3}{4} \cdot\left(\frac{R}{\lambda}\right)^{-2} \cdot \frac{F_{n}}{A_{0}}
$$

When adopting the same values for normal load and nominal contact area as in Paragraph 2.1 ( $2 \mathrm{~N}$ and $60 \mathrm{~mm}^{2}$ respectively), for $R / \lambda=0.017$, the smallest value of all samples used in the present study, an equivalent elastic modulus of the skin is required that exceeds $86 \mathrm{MPa}$. Pailler-Mattei et al. [15] and Yuan and Verma [13] report values for the stratum corneum elastic modulus obtained from indentation measurements, which for dry skin exceed the value of $86 \mathrm{MPa}$. From this it may be concluded that the assumption of elastic behaviour at the scale of the surface features is valid.

\subsection{Friction of metal samples}

The coefficient of friction measured of metal samples against the fingerpad is plotted as a function of normal load in Fig. 5. The frictional behaviour of a smooth, non-textured, metal sample was measured for reference.



Figure 5. Coefficient of friction as a function of normal load for metal samples. 


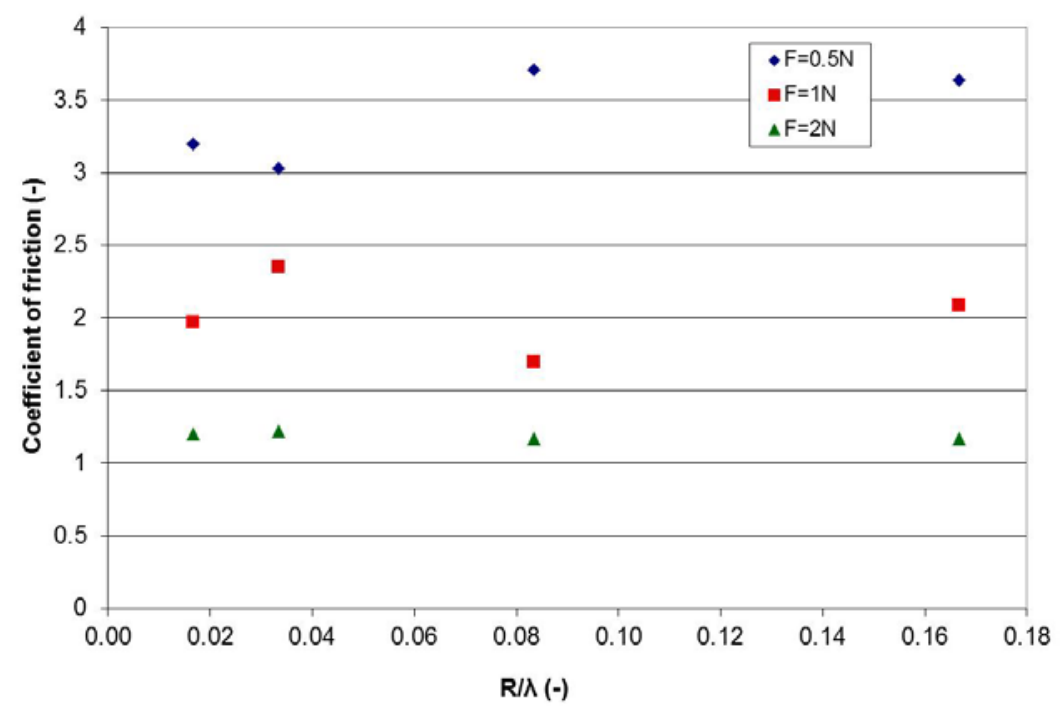

Figure 6. Coefficient of friction as a function of texture parameter $R / \lambda$ for metal samples.

Since we know from e.g. Adams et al. [7] and the theory in Section 3.1 that adhesion is the dominant mechanism in the majority of contacts involving human skin, from Equation (5) a clear relation between the coefficient of friction and $R / \lambda$ is expected. The theory of adhesion being the dominant mechanism is supported by the dependence of the coefficient of friction on the normal load, as shown in Fig. 5. For the smooth surface, the coefficient of friction decreases with normal load following a power law with power -0.36. Assuming that adhesion is the dominant friction mechanism the measured friction force is directly proportional to the real contact area, so that the coefficient of friction is given by

$\mu=\frac{\tau \cdot A_{r}}{F_{N}}$

where the real contact area $A_{r}$ is known to depend on the normal load following $A_{r} \propto F_{N}{ }^{a}$

The coefficient of friction between the skin of the fingerpad and a smooth surface is thus expected to depend on the normal load as

$\mu \propto{F_{N}}^{a-1}$

From the experimental results for $F_{N}$ an exponent $a-1=-0.36$ was measured, so in equation (10) $a=0.64$. This value corresponds well to the exponent $0.68 \pm 0.09$ found by Soneda and Nakano [12] for normal loads between 0.1 and $5 \mathrm{~N}$.

A nonlinear decrease of the coefficient of friction with normal load is observed for random roughness as well $[5,16,17]$. The coefficient of friction of the textured surfaces is related with normal force through a power law with the power $n$ ranging from -0.83 to -0.65 , which is much stronger than we would expect from Equation (5). From this equation and Equation 
(10) describing the relation between contact area and normal load the coefficient of friction is expected to depend on the normal load following

$\mu \propto\left(\frac{A_{0}}{F_{N}}\right)^{1 / 3} \propto F_{N}^{1 / 3(a-1)}$

Since the contact area increases with normal load the exponent in Equation (10) $a>0$, so according to Equation (12) the coefficient of friction of the textured surfaces will depend on the normal load with an exponent $n>-1 / 3$. However, an exponent $n$ ranging from -0.83 to 0.65 was measured. The strong dependence of the measured coefficient of friction on the applied normal load is expected to be caused by normal adhesion, which increases the effective normal load on the contact spots. The measured friction load $F_{\mu \text {,meas }}$ is related to the applied normal load $F_{N}$ through the measured coefficient of friction $\mu_{\text {meas }}$

$F_{\mu, \text { meas }}=\mu_{\text {meas }} \cdot F_{N}$

The measured coefficient of friction can also be expressed as the product of the coefficient of friction $\mu$ and the effective normal load at the contact area which is composed of the applied normal load $F_{N}$ and the load due to normal adhesion $F_{a d h}$ :

$F_{\mu, \text { meas }}=\mu \cdot\left(F_{N}+F_{a d h}\right)$

The measured coefficient of friction thus depends on the applied normal load following

$\mu_{\text {meas }}=\frac{F_{\mu, \text { meas }}}{F_{N}}=\mu \cdot\left(1+\frac{F_{a d h}}{F_{N}}\right)$

which expression also shows that the relative contribution of adhesion decreases with increasing normal load.

Figure 6 shows the coefficient of friction as a function of texture parameter $R / \lambda$. For the surface texture parameter $R / \lambda$ no relation with the coefficient of friction can be determined from Fig. 6. From Equation (5) the coefficient of friction would be expected to increase with increasing parameter $R / \lambda$. However, up to this point the equivalent elastic modulus is assumed to have a constant value. From indentation measurements (e.g. Yuan and Verma [13]) a clear length-scale effect can be observed: The effective elastic modulus decreases with increasing indentation depth at constant indenter radius and decreases with decreasing indenter radius at constant indentation depth. An increase of the surface parameter $R / \lambda$ thus involves an increase in effective elastic modulus $E^{*}$. The total effect of these mechanisms depends on the magnitude of radius, spacing and normal load, but observation of Equation (5) reveals that an increase in both $R / \lambda$ and $E^{*}$ conceals a clear effect of the surface texture parameter.

\subsection{Friction of TPU samples}

Figure 7 shows the coefficient of friction as a function of normal load. Figure 8 shows the coefficient of friction as a function of texture parameter $R / \lambda$ for dry and hydrated skin. 


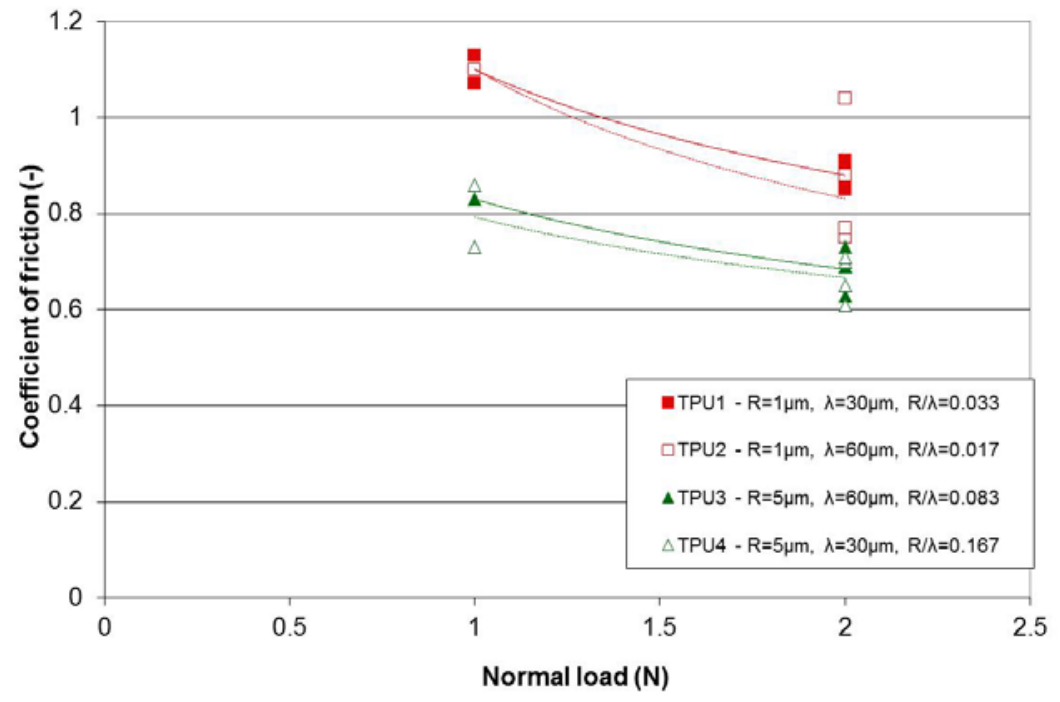

Figure 7. Coefficient of friction as a function of normal load for TPU samples.

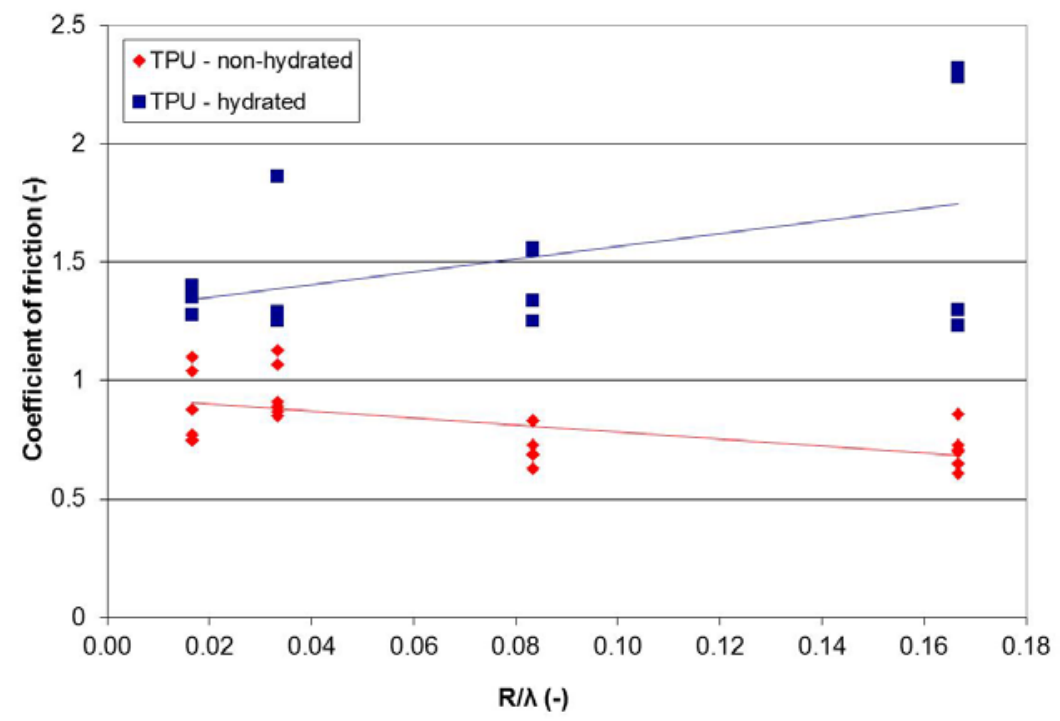

Figure 8. Coefficient of friction as a function of texture parameter $R / \lambda$ and for TPU samples sliding against non-hydrated and hydrated skin.

From Figs. 7 and 8 a decrease of the coefficient of friction with surface texture parameter $R / \lambda$ can be observed for non-hydrated skin. From Fig. 6 it can be seen that this decrease is mainly a dependence on tip radius $R$. The injection moulded textures with tip radii $R=5 \mu \mathrm{m}$ have a 
two-dimensional waved surface, whereas the textures with tip radii $R=1 \mu \mathrm{m}$ have a grated pattern with small-scale surface features superposed on this pattern as shown in Fig. 3 . The theory proposed in paragraph 2.1 assumes the contact area being built up from Hertzian contacts at the asperity level, which applies for the two-dimensional waved surface with tip radius $R=5 \mu \mathrm{m}$. For the textures with tip radii $R=1 \mu \mathrm{m}$ the grating is in contact with the skin as well, demanding a more complicated contact model as discussed in paragraph 3.1.1. Comparison of the mechanisms responsible for the frictional behaviour of these two different sets of textures is therefore considered irrelevant within the scope of this work.

Figure 8 shows the difference in frictional behaviour between non-hydrated and hydrated skin. The hydration level of non-hydrated skin during the test program was $70 \pm 8 \mathrm{AU}$, for hydrated skin it was higher $110 \pm 6$ AU. This increase in hydration level of the skin of the fingerpad corresponds to observations by Verrillo et al. [11], who reported an increase of $35 \%$ after submersion in water. This relatively small increase in hydration level as compared to the differences observed at other anatomical sites is due to the relatively high baseline level of the fingerpad. The biological function of this higher level of hydration is to increase the coefficient of friction during manipulating tasks. Coefficients of friction for the nonhydrated fingerpad range from 0.6 tot 1.1. For the hydrated fingerpad coefficients of friction between 1.25 and 2.3 were measured. The increase in friction with the skin surface hydration level is mainly caused by softening of the stratum corneum. The increase of friction with hydration measured against TPU corresponds to earlier observations by e.g. Derler et al. [5], Sasada [16] and André et al. [17, 18].

\section{Conclusions}

Friction of well-defined regular surface textures produced with picosecond laser pulses was measured against the skin of the fingerpad. The coefficient of friction decreased strongly with normal load for both metal and TPU surfaces supporting the theory that adhesive friction is the dominant friction mechanism. The strong dependence of the measured coefficient of friction on the applied normal load is expected to be caused by normal adhesion, which increases the effective normal load on the contact spots.

Experiments with TPU against hydrated skin showed an increase of the coefficient of friction with a factor two compared to dry skin. Within the range of surface feature geometries investigated, no clear relation was found between feature geometry and coefficient of friction. This is probably due to scale-effects of the elastic behaviour of the stratum corneum counteracting with the influence of variation in feature geometry.

Fabrication of surface structures by laser texturing using picosecond laser pulses has proven to be a useful technique for producing well-defined micro-scale surface textures. The control of texture parameters for the injection moulded surfaces needs further work. The frictional behaviour of these micro-scale textures is determined by the properties of the stratum 
corneum. Considering that the stratum corneum has a high elastic modulus optimization of textures produced by laser texturing is expected to bring forth surfaces having very low friction.

Further work will involve a parameter study to gain more understanding of the relation of surface feature geometry and scale-effects in the elastic behaviour of the stratum corneum. An experimental program involving a larger number of subjects should give insight in the robustness of the theories describing the frictional behaviour.

Furthermore, this study will be extended to measuring the response of subjects to the different surface textures to provide a basis for calculation of the 'feel' of these surfaces.

\section{Acknowledgements}

This research was carried out under the project number MA.08113 in the framework of the Research Program of the Materials innovation institute M2i (www.m2i.nl).

\section{References}

[1] Barnes CJ, Childs THC, Henson B, and Southee CH. Surface finish and touch - a case study in a new human factors tribology. Wear 2004;257:740-50.

[2] Childs THC and Henson B. Human tactile perception of screen-printed surfaces: selfreport and contact mechanics experiments. Proc IMechE Part J: J Eng Trib 2007;221:427-41. [3] Liu X, Yue Z, Cai Z, Chetwynd DG and Smith ST. Quantifying touch-feel perception: tribological aspects, Meas Sci Tech 2008;19:08007-1-9.

[4] Etsion J, State of the art in laser surface texturing, J of Tribology, Trans ASME 2005;127:248-53.

[5] Derler S, Gerhardt L-C, Lanez A, Bertaux E and Hadad M. Friction of human skin against smooth and rough glass as a function of the contact pressure. Trib Int 2009;42:156574.

[6] Skedung L, Danerlöv K, Olofsson U, Aikala M, Niemi K, Kettle J and Rutland MW. Finger friction measurements on coated and uncoated printing paper, Trib Letts 2010;37:38999.

[7] Adams MJ, Briscoe BJ and Johnson SA. Friction and lubrication of human skin. Trib Letters 2007;263:239-53.

[8] Römer GRBE, Huis in’t Veld AJ, Meijer J, Groenendijk MNW. On the formation of laser induced self-organizing nanostructures, CIRP Annals 2009 - Manuf Techn;58:201-204.

[9] Sivamani RK, Goodman J, Gitis NV and Maibach HI. Friction coefficient of skin in realtime. Skin Res Tech 2003;9:235-9.

[10] Gerhardt L-C, Strässle V, Lenz A, Spencer ND and Derler S. Influence of epidermal hydration on the friction of human skin against textiles. J R Soc Interface 2008;5:1317-28. 
[11] Verrillo RT, Bolanowski SJ, Checkosky CM and McGlone FP. Effects of hydration on tactile sensation. Som Mot Res 1998;15:93-108.

[12] Soneda $\mathrm{T}$ and Nakano K. Investigation of vibrotactile sensation of human fingerpads by observation of contact zones. Trib Int 2009;43:210-7.

[13] Yuan Y and Verma R. Measuring microelastic properties of stratum corneum. Coll Surf B: Bioint 2006;48:6-12.

[14] Wu KS, Van Osdol, WW and Dauskardt RH. Mechanical properties of human stratum corneum: Effects of temperature, hydration, and chemical treatment. Biomat 2006;27:785-95. [15] Pailler-Mattei C, Pavan S, Vargiolu R, Pirot F, Falson F and Zahouani H. Contribution of stratum corneum in determining bio-tribological properties of the human skin. Wear 2007;263:1038-43.

[16] Sasada, T. The friction of human skin, In: Proc $21^{\text {st }}$ Biotrib Symp Fukuoka Japan; 2000, p.46-52.

[17] André T, Lefèvre P and Thonnard J-L. A continuous measure of fingertip friction during precision grip. J Neurosci Meth 2009;179:224-9.

[18] André T, De Wan M, Lefèvre P and Thonnard J-L. Moisture Elevator: a direct measure of fingertip skin hydration during object manipulation. Skin Res Tech 2008;14:385-9. 


\section{Paper E}

Touch properties of surfaces: How to measure tactile friction in vivo

J. van Kuilenburg, M.A. Masen and E. van der Heide

Submitted to Materials \& Design 



\title{
Touch properties of surfaces: How to measure tactile friction in vivo
}

\author{
J. van Kuilenburg, M.A. Masen and E. van der Heide
}

\begin{abstract}
The friction behaviour one observes when exploring a surface by touching is susceptible to sources of variation which are intrinsic to tactile exploration. This paper reports on the results of an experimental study to investigate the sources of this variation. The variation of the observed friction can be explained by the contact mechanics of the fingertip. Furthermore, the frictional behaviour of the skin changes with hydration of the skin. Under conditions where adhesion plays a role, such as with smooth surfaces or textures with high asperity densities, variations in hydration and cleanliness of the skin will result in considerable variations in the adhesion force and thus in variations in the observed friction. Considering the human aspects of the tactile friction measurement will enable researchers to draw the correct conclusions on the relation between physical properties, frictional behaviour and perceived qualities of the surface.
\end{abstract}

\section{Keywords}

Touch perception, friction experiments, surface geometry, skin tribology

\section{Introduction}

Optimization of the tactile properties of product surfaces is frequently achieved through trial and error. Recently, the development of guidelines which will enable product developers in industry to predict and optimize the emotional qualities and expectations associated with specific surface finishes has become the subject of tribological research [1-7]. Tribology being the science of friction, adhesion, lubrication and wear. From the viewpoint of the tribologist, it is the relation between the sensorial and affective responses, frictional behaviour and the surface properties of the material that is of most interest. The sensorial responses, also psychophysical responses, can be related almost directly to physical measurements of material and surface properties. Perceptions of smoothness, warmth, softness 
and slipperiness can be related to measures of roughness, thermal conductivity, compliance and friction [8] using statistical techniques. While the first three properties can be measured directly from the material using various types of advanced equipment, measuring the friction behaviour requires two surfaces, one of which is a living subject. Although several studies investigated the psychophysical relation between touch properties and friction [9-11] the relation between the perceived intensity, slipperiness, and the physical intensity, friction, still needs research [8]. Besides the direct relation which exists between the perceived and physical intensity of friction, the friction that occurs between the skin and a surface plays an important role in the perception of smoothness. Whereas smoothness and slipperiness are the expertise of a psychophysiologist, friction is the expertise of the tribology engineer.

Numerous studies have been reported on the friction behaviour of the human fingerpad. A wide range of coefficients of friction is presented as a function of countersurface material, surface roughness, normal load and skin condition as shown in a review on skin friction studies by Derler and Gerhardt [12]. Different methods have been used to measure the frictional forces in skin-object interactions. A well-accepted method for measuring tactile friction is the principle of a stationary sample that is attached to a load measuring device, over which the subjects slide their fingerpads [13-18]. The execution of these friction experiments is easy, so that several researchers used this principle in combination with questionnaire research to investigate the relation between friction properties and the various aspects of tactile perception $[2,4-6]$. When carrying out experiments in vivo, the condition of the living skin will depend on the temperature and humidity of the environment, which are seldom controlled, the time of day and other personal aspects [19]. Obviously, this variation in conditions will lead to a variation in measured coefficients of friction. To be able to draw the correct conclusions on the relation between the surface parameters and frictional behaviour of the skin, one needs to understand the influence of conditions such as the measuring method and skin cleanliness and hydration level. This work reports on the results of an experimental study to investigate the sources of this variation of the measured friction, with the aim to give recommendations for an experimental procedure for measuring tactile friction. Furthermore, a concise theoretical background is provided for the correct interpretion of the friction results, while taking into account any effects caused by varying measurement conditions. 


\section{Methods and materials}

\subsection{Methods}

\subsubsection{Friction measurement}



Figure 1. Experimental set-up for measuring the sliding friction between the fingerpad and textured samples.

In this study a set-up was used, which was discussed earlier by Masen [17]. Sliding friction between the fingerpad and the samples was measured using a load cell model ATI Gamma (ATI Industrial Automation, USA) as shown schematically in Fig. 1. Resolution of the 3 -axis force/torque sensor is $25 \mathrm{mN}$ in the normal direction and $12.5 \mathrm{mN}$ in tangential direction, with a sample frequency of $100 \mathrm{~Hz}$. Samples were attached to the load cell using double-sided adhesive tape. Measurements were carried out by sliding the index finger over the sample in the horizontal plane. The normal load can be applied by pressing the finger onto the sample, while visually monitoring the measured normal load on the computer display. However, improved load stability has been observed when a dead weight is attached to the finger. In this study, weights of $100 \mathrm{~g}$ and $200 \mathrm{~g}$ respectively were attached to the finger using double-sided adhesive tape, yielding normal loads of approximately $1 \mathrm{~N}$ and 2 N. Normal loads exceeding this value were applied actively by the subject, because these 
weights were too large to attach to the finger. The sliding velocity was approximately 10 $\mathrm{mm} / \mathrm{s}$. All tests were carried out at a room temperature of $20 \pm 2^{\circ} \mathrm{C}$ and relative humidity $45 \pm 5 \%$.

The coefficient of friction was calculated as the quotient of the measured friction force $F_{\mu}$ and normal load $F_{n}$ following

$$
\mu=\frac{F_{\mu}}{F_{n}}
$$

The normal load equals the load measured in vertical direction $F_{n}=F_{z}$, whereas the friction load is calculated from the loads measured in the lateral $x$ - and $y$-directions following

$$
F_{\mu}=\sqrt{F_{x}^{2}+F_{y}^{2}}
$$

The coefficient of friction of a measurement is the average friction coefficient from one single stroke carried out backwards, i.e. towards the body, measured during the steady state part of the sliding motion. Fig. 2 shows an example of a single friction measurement. Steady state means that data around the start and end points of the motion is omitted.

Experiments on various samples were carried out in random order. All measurements were repeated five times, on different days and times, within a period of two months to gain insight in reproducibility of the results. Standard deviations were calculated for single measurements as well as for sets of different experiments following

$$
\sigma=\sqrt{\frac{\sum\left(\mu_{i}-\mu_{\text {mean }}\right)^{2}}{n}}
$$

\subsubsection{Skin preparation}

Before the experiments the hands were washed using water and soap and air-dried. After a rest period of approximately $10 \mathrm{~min}$ to allow the washed skin to regain its normal condition, the experiments were carried out. Experiments on hydrated skin were carried out after submersion in water for at least $10 \mathrm{~min}$. Verrillo et al. [20] showed that after submersion from 5 to 10 min hydration returns to normal levels between 5 and 15 min, much longer than the time needed for one experiment. Before the experiments excess water was removed using a paper tissue. Before and during the measurements the hydration level of the skin surface was monitored using a Corneometer CM 825 (Courage+Khazaka GmbH, Germany). The Corneometer measures the changing capacitance of a precision capacitor in arbitrary 


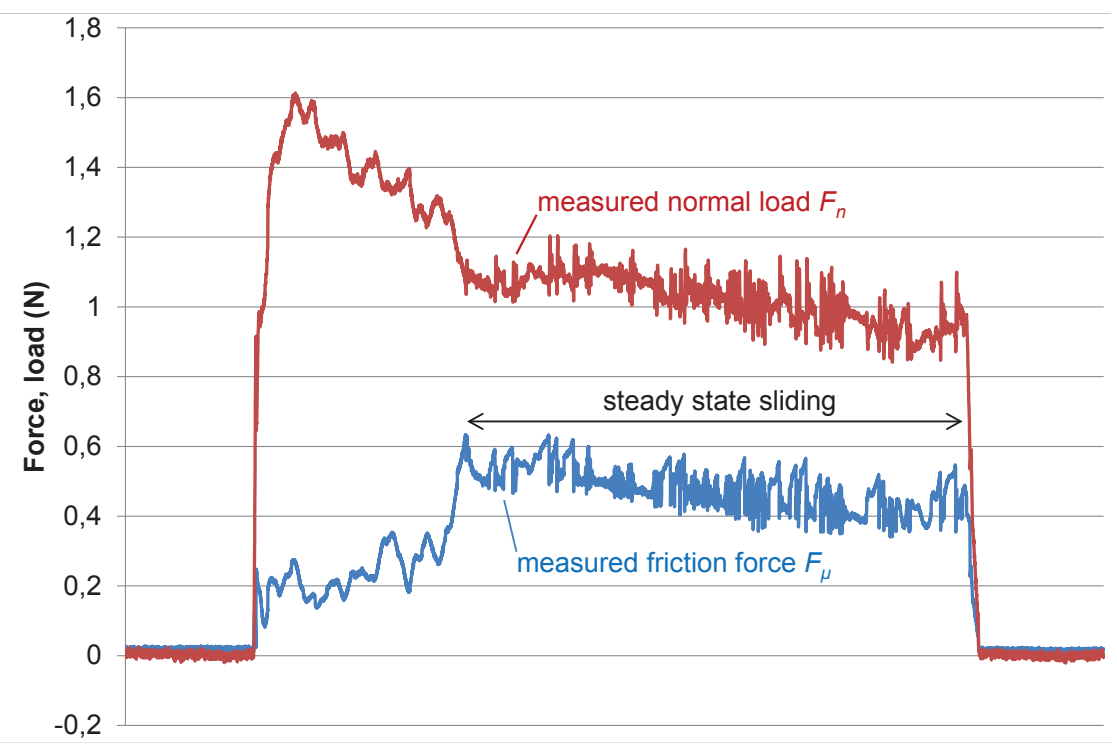

Figure 2. Example of a single measurement result.

units AU, which represents the change in the dielectric constant due to hydration of the skin surface. The hydration of the skin has been linked to increasing friction by e.g. Cua et al. [21], Gerhardt et al. [22] and Kwiatkowska et al. [23].

\subsection{Materials}

In vivo experiments were carried out by sliding the fingerpad against surfaces with regular surface textures and against sandpaper surfaces.

\subsubsection{Skin}

In vivo experiments were carried out using the fingerpad of the left (dominant) index finger of a 38-year-old male subject. For comparison, some experiments were carried out using the middle and ring finger of the left hand of the same subject. In its normal state, i.e. untreated, the skin may be referred to as dry. Damp skin is the skin after submersion and wiping of free water. With damp skin, the top layer of the skin is hydrated, while there is no free water left on the surface of the skin. Hydration of the stratum corneum, the top layer of the skin, influences its physical and mechanical properties, and hence the observed friction behaviour[24-26]. With the hydration level increasing, the elasticity of the stratum corneum reduces by several orders of magnitude, leading to an increase of the contacting area and hence of the measured friction $[10,12,13,17,19]$. 


\subsubsection{Surface textures}



Figure 3. SEM picture of a laser-textured sample with a tip radius $R=5 \mu \mathrm{m}$ and spacing $\lambda=50 \mu \mathrm{m}$ (courtesy of Lightmotif, the Netherlands).

The complicated scale dependence of roughness makes it difficult to pinpoint the feel to a certain geometric parameter [1]. The amplitude parameters which are commonly used to characterize a surface's roughness are in fact statistical parameters describing the height distribution of the measured surface profile. Those parameters do not contain any information about size, shape or density of the asperities. Besides, when investigating friction of rough surfaces, a common approach is the investigation of the friction behaviour of a single asperity as a first step. Because of the motion of the skin caused by for example blood circulation, carrying out experiments in vivo at the level of a single asperity, which is generally at the micrometer scale, is deemed impossible. By producing well-defined surface topographies these obstacles can be avoided [2].

To investigate the friction behaviour as a function of asperity geometry, surface textures consisting of evenly distributed spherically-tipped asperities were produced using ultrashort pulsed laser technology (Lightmotif, the Netherlands). The textures were applied onto flat stainless steel samples measuring 20 by $40 \mathrm{~mm}$. The surface textures are composed of bumps having a spherical tip with radius $R$, which are evenly distributed with a spacing $\lambda$ between the tips [27]. A series of 5 samples was produced with tip radii of 2,5 , and $10 \mu \mathrm{m}$ and spacings varying between 20 and $100 \mu \mathrm{m}$. The samples are characterized by 
a texture parameter $R / \lambda=2 / 20,5 / 20,5 / 50,5 / 100$ and 10/100. Fig. 3 shows a SEM picture of one of the samples. The height of the the asperities is $20 \pm 2 \mu \mathrm{m}$, the height of the saddle points is $10 \pm 2 \mu \mathrm{m}$. Surface topography was measured using a 3D Laser Confocal Microscope model VK-9700 (Keyence, USA). Tip radii of the asperities were obtained from line scans of the 3D surface profile.

\subsubsection{Sandpaper}

A sample material commonly used to investigate perceived roughness is sandpaper, see for instance $[20,28]$. Furthermore, a positive correlation appears to exist between perceived roughness and the measured coefficient of friction [8]. Friction experiments were carried out using different grades of sandpaper. 3M Wetordry Tri-M-ite abrasive paper was used in different grades between p 80 and p2000, so that average particle diameters varied from 201 down to $10.3 \mu \mathrm{m}$. The silicon carbide grit is bonded with a waterproof resin and covers $100 \%$ of the closed coat paper. Fig. 4 shows a microscopic image of a sandpaper sample.

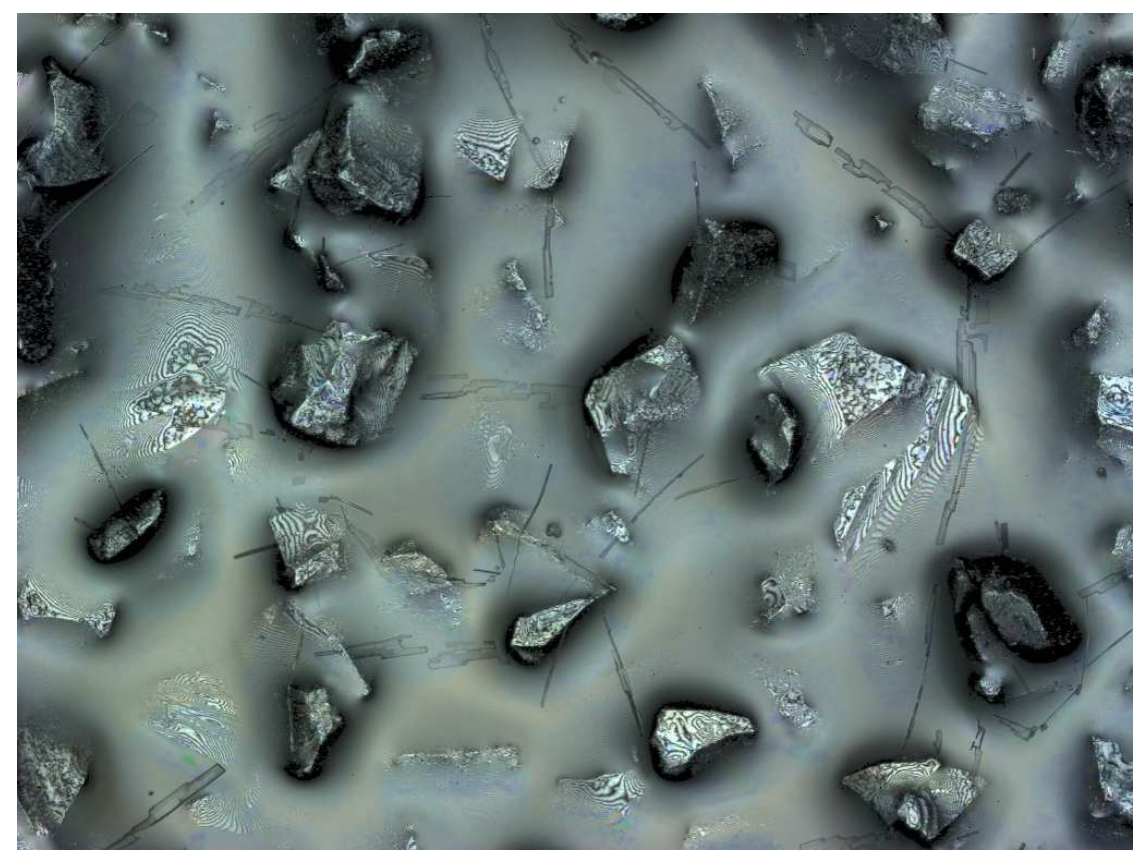

Figure 4. Microscopic picture of a p600 grade sandpaper sample (50× magnification). 


\section{The influence of measurement conditions}

As described in Paragraph 2.1 the tactile friction experiment is carried out in a, preferably controlled, environment which can be characterized by an ambient temperature and relative humidity. These characteristics, together with the bodily state of the subject, determine the condition of the skin. The actual measurement is then executed by positioning a finger on the sample surface of interest and applying a predefined normal load, whereupon a sliding motion of the finger over the surface yields the desired friction signal. When interpreting the measurement results, the variation of measurement conditions, which are characteristic of in vivo experiments, should be taken into account.

\subsection{Hydration level}

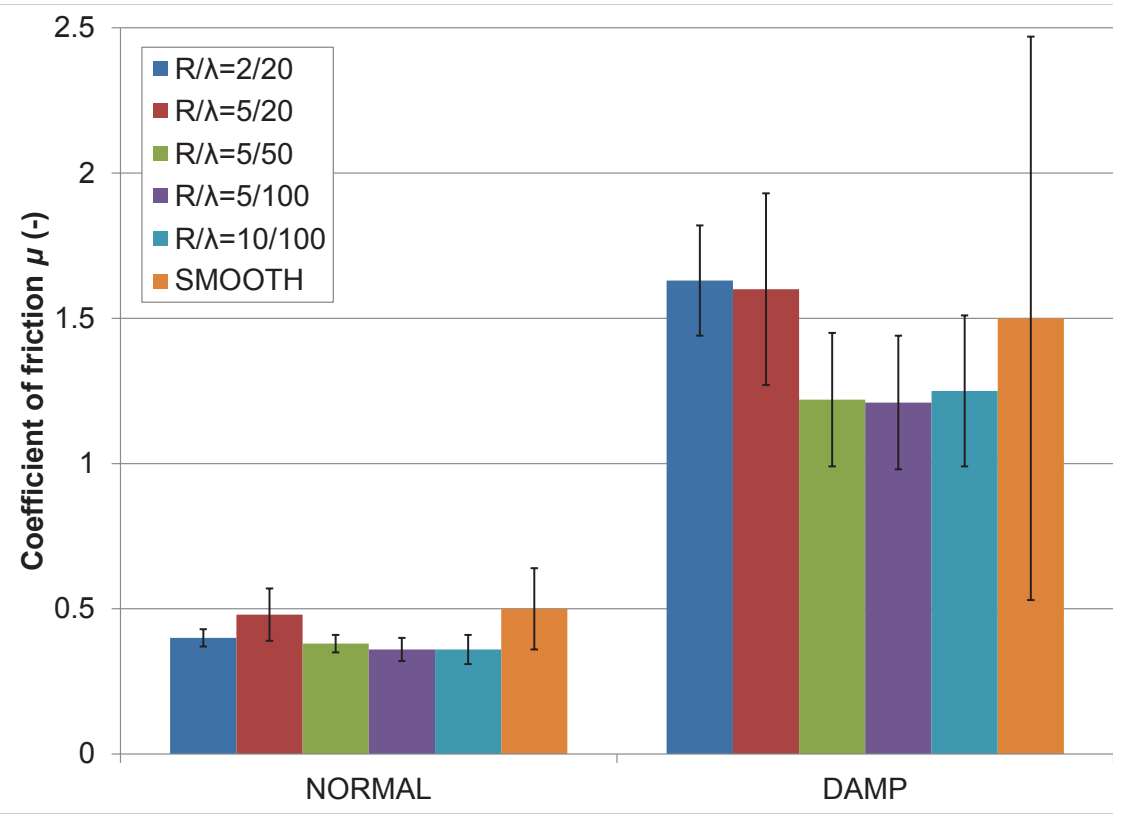

Figure 5. Coefficient of friction for normal and damp skin of the index finger at $1 \mathrm{~N}$ normal load for different texture parameters.

Most of the variation found in the coefficient of friction can be explained by differences in the hydration level as found by, for example, Veijgen et al. [19] in a large study involving over 30 participants. Fig. 5 shows the coefficient of friction measured against textured samples for normal and damp skin. The average hydration level during the tests was 42 AU for normal skin, within the range 34-50 AU, and 97 AU for damp skin, within the range 77-108 AU. The coefficient of friction measured against damp skin is considerably 
larger than against normal, or dry, skin. For damp skin the coefficient of friction decreases from about 1.6 at $20 \mu \mathrm{m}$ tip spacing to about 1.2 at 50 and $100 \mu \mathrm{m}$ and is independent of tip radius. The increase is more than threefold when compared to the skin in its normal state.

\section{$3.2 \quad$ Finger}

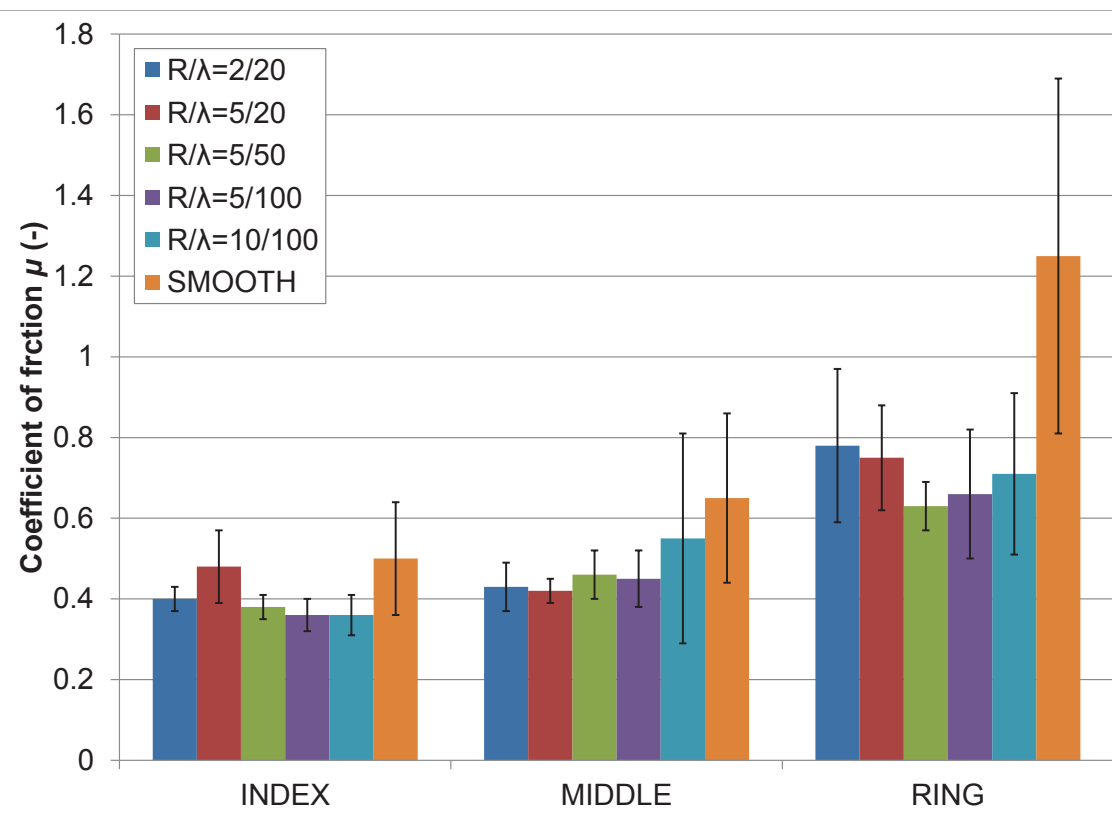

Figure 6. Coefficient of friction of untreated skin for different fingers at $1 \mathrm{~N}$ normal load for different texture parameters.

Since in general we explore surfaces with more fingers than just the index finger, the variation in frictional response has been evaluated. Fig. 6 compares the frictional behaviour as a function of texture characteristics for the index, middle and ring finger of the left hand of a 38-year-old subject. The skin of the fingers is in its normal state. It appears that the friction measured against the ring finger is considerably higher than the friction measured against the other fingers. The average hydration level of the ring finger during the tests was $66 \mathrm{AU}$, as opposed to 42 and $51 \mathrm{AU}$ for the index and middle finger respectively. Furthermore, the obvious difference in geometry of the different fingertips causes a variation in the measured friction behaviour between the different fingers. 


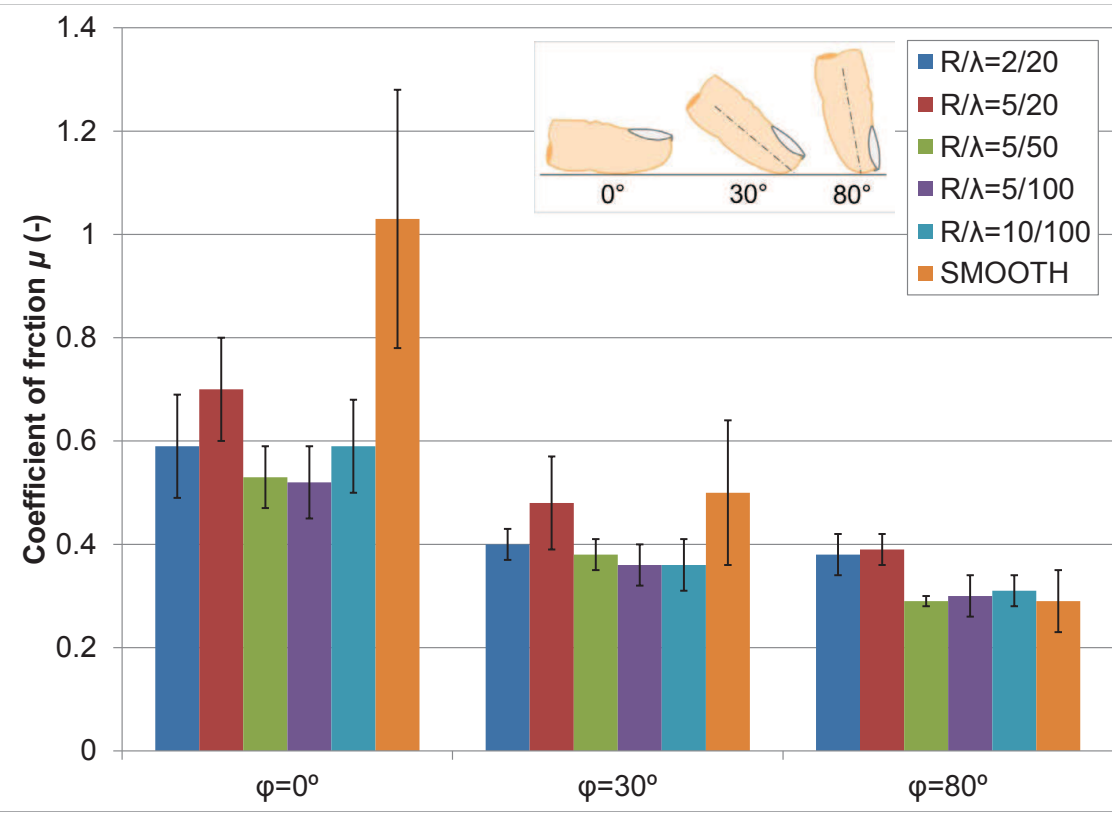

Figure 7. Coefficient of friction of the index finger for different orientation angles of the finger at $1 \mathrm{~N}$ normal load for different texture parameters.

\subsection{Orientation angle}

While sliding over a surface, the finger might have different orientations varying, for instance, from parallel to the surface using the fingerpad to perpendicular using the fingertip. Fig. 7 shows the coefficient of friction as a function of texture characteristics and orientation of the fingerpad. The figure shows that the coefficient of friction increases significantly with decreasing orientation angle. The orientation angle being defined as 0 for the fingerpad and $80^{\circ}$ for the fingertip as defined in Fig. 1. Warman and Ennos [29] and Tomimoto [30], who measured the friction force of the fingerpad as a function of normal load up to $2 \mathrm{~N}$ for different orientation angles, observed that the coefficient of friction decreased with increasing orientation angle of the finger. The decrease in measured friction with increasing orientation angle can be attributed to the reduction of the contact area with increasing angle.

\subsection{Normal load}

Based on data from the literature, in grip-related experiments, where normal load ranges up to $20 \mathrm{~N}$ or more are common, the coefficient of friction is expected to depend significantly on normal load. However, when touching a surface to assess its tactile properties, relatively low loads are applied within a limited range. Fig. 8 gives an overview of the results for 


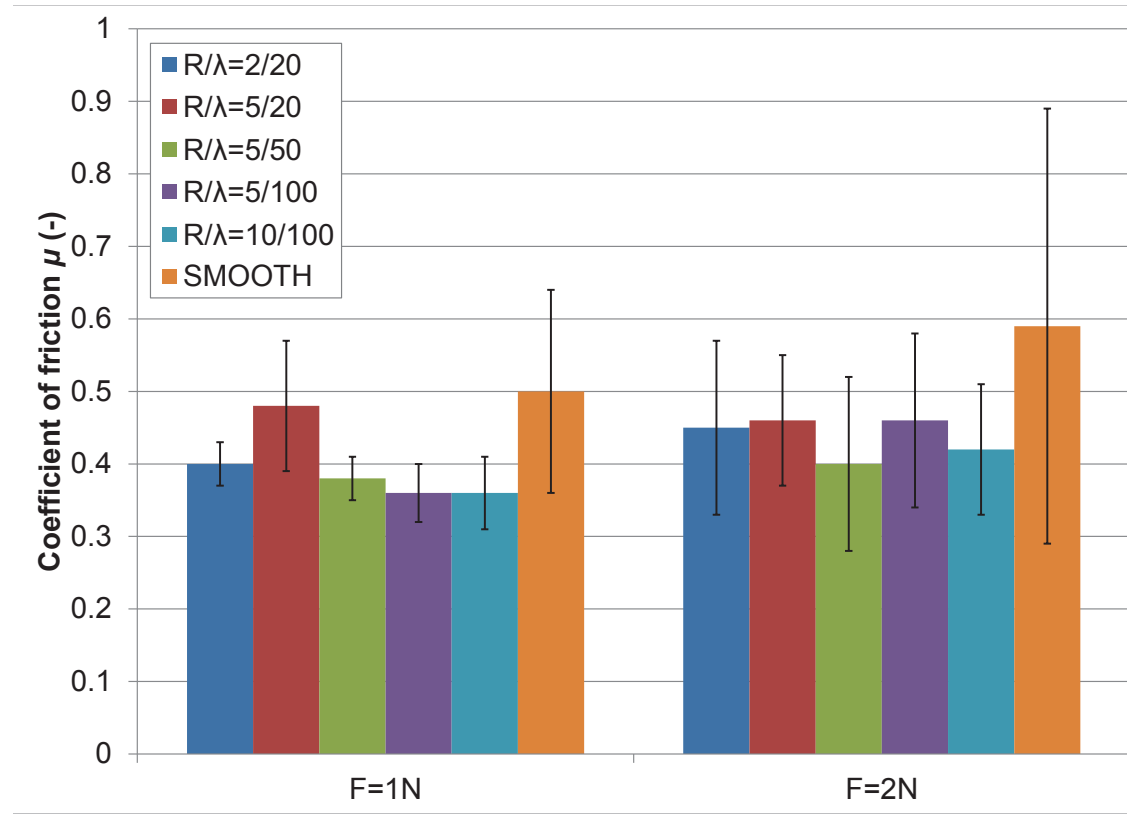

Figure 8. Coefficient of friction of the index finger as a function of normal load for different texture parameters.

different normal loads. Obviously the load range, increasing from 1 to $2 \mathrm{~N}$, is relatively small. There is no significant difference between the normal loads 1 and $2 \mathrm{~N}$. Several studies show that as a function of normal load the measured coefficient of friction follows a power law with exponents ranging between -0.28 and -0.22 within a load range 0 to $2 \mathrm{~N}$ $[29,31]$ and 0 to $12 \mathrm{~N}$ [32]. Some authors report a linear dependence of the friction force on normal load, which corresponds to a constant coefficient of friction $[2,33]$. In touch experiments, at normal loads ranging from 1 to several Newtons, any variations in normal load will not contribute to significant variations in the coefficient of friction.

\subsection{Direction of motion}

Several authors report a dependence of the measured coefficient of friction on the sliding direction $[14,30]$. Fig. 9 shows there is a slight dependence of the measured friction on the stroking direction, the coefficient of friction tending towards lower values in the forward direction. Although the average coefficient of friction was observed to be lower, in the forward direction the sliding of the fingerpad appeared to be prone to stick-slip behaviour. The intermittent sticking, which was clearly noticed during the measurements, causes an irregular measurement signal characterized by large differences in friction between the sticking and the sliding phases. 


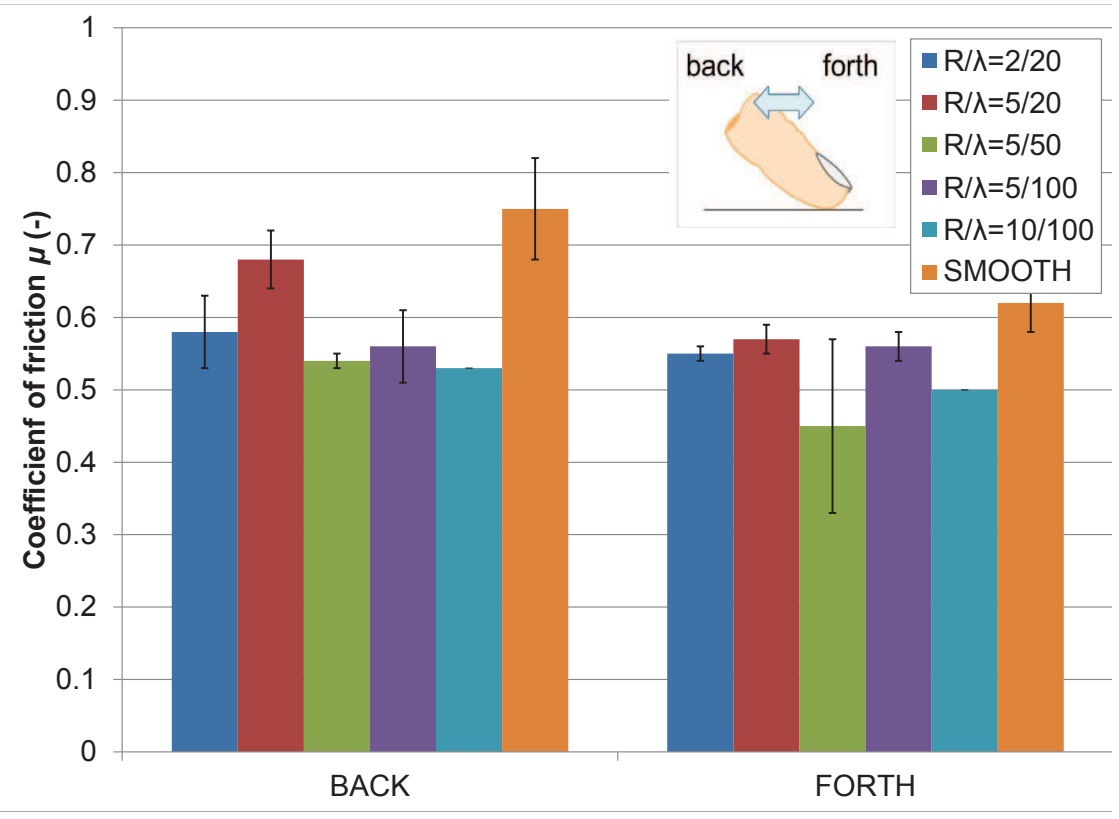

Figure 9. Coefficient of friction of the index finger for sliding directions back and forth.

\subsection{Repeats}

During the experiments sweat, dead cells and possibly other contamination are transferred from the skin of the fingerpad onto the countersurface. Fig. 10 shows the coefficient of friction measured against the fingerpad as a function of the number of repeats. The coefficient of friction appears to decrease with the number of repeats. However, after 20 repeats the coefficient of friction reaches a constant value. Several authors observed the transfer of lipid material from the finger to the sample surface [13, 33, 34]. Multiple use of test samples demands a thorough cleaning to remove this transfer layer, as it influences the friction between the two surfaces. This is achieved by cleaning all samples ultrasonically using ethanol before and in between the experiments.

\subsection{Sources of variation}

The standard deviation of the measurements carried out between the textured samples and dry skin varies between approximately 10 and $20 \%$. The average coefficient of friction was obtained from measurements involving the left index finger of a single subject. The only planned variation in test conditions was the time and day: measurements were carried out on different days within a period of two months, at different times of the day. Analysis of the measurement data revealed that the variation of the normal load is small. The 


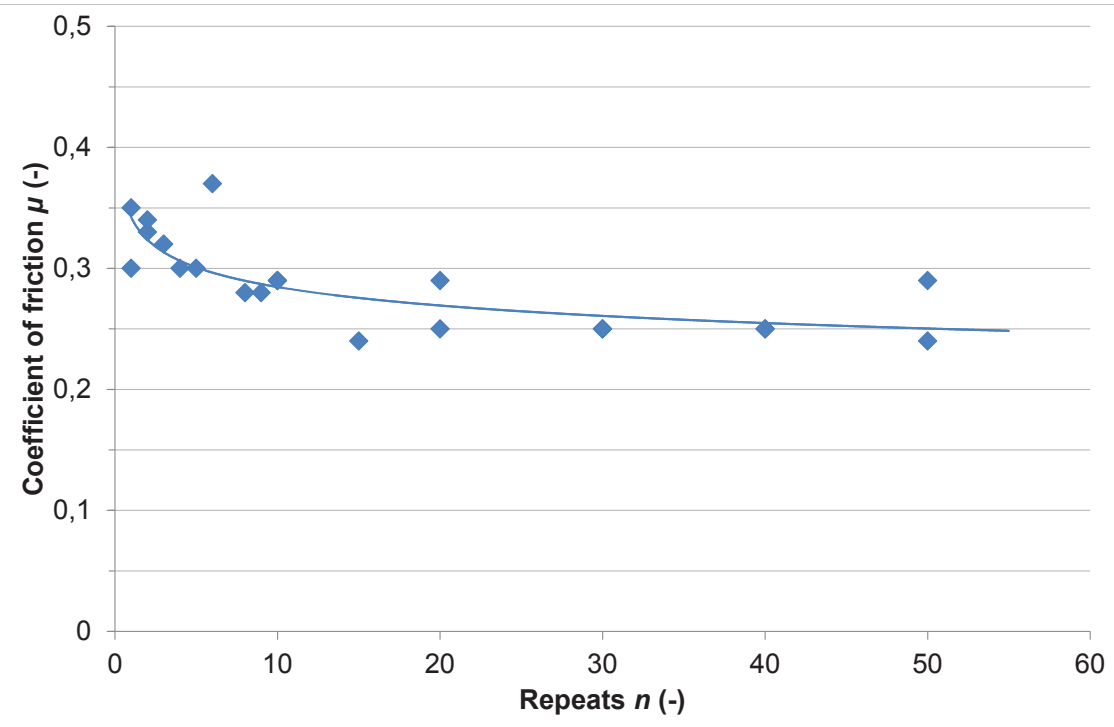

Figure 10. Coefficient of friction between the normal skin of the index finger and a textured sample at $1 \mathrm{~N}$ normal load as a function of the number of repeats.

average normal load is $1.23 \mathrm{~N}$ with $21 \%$ standard deviation. When taking into account the minor effect the normal load has on the friction within the range 1 to $2 \mathrm{~N}$, the influence of variation of normal load on the measured friction is considered to be negligible within this load range, as shown in Paragraph 3.4. The average hydration level measured during the test program is $42 \mathrm{AU}$, with a minimum of $34 \mathrm{AU}$ and a maximum of $49 \mathrm{AU}$. Even though this range is considered too small to be of influence, as shown in Paragraph 3.1, closer observation of the results shows that there is no correlation between the measured hydration level and the friction of skin in its normal state. In this study the intended orientation of the fingerpad is approximately $30^{\circ}$. However, in practice the angle varies between $20^{\circ}$ and $40^{\circ}$, so that the orientation of the fingerpad is expected to contribute considerably to the observed variation in test results.

When the friction is high a relatively high variation between measurements can be observed, for instance when sliding against smooth samples, Fig. 8, and when the skin is damp, Figs. 5 and 6 . In these cases, during several of the individual experiments stick-slip phenomena were observed. During the sticking phase, the friction is considerably higher than during steady sliding, which eventually leads to a large variation in the reported averaged values for the coefficient of friction. One may conclude that, when investigating the relation between friction and tactile perception, the measuring signal contains relevant information. Smith et al. [11] found that the rate of variation in measured friction force plays a significant role in the perception of roughness of grated patterns. More recently, 
Fagiani et al. [31] studied the characteristics, frequency spectra and amplitudes of the vibrations, which are generated in the fingertip when sliding over different surfaces.

It can be concluded that there are many causes of variations in the observed friction behaviour. Many of these are intrinsic to the tactile exploration of product surfaces. However, the real subject of interest is the role of the product surface properties, such as the surface microgeometry, on the friction behaviour.

\section{Influence of the countersurface}

\subsection{Friction between the skin and a textured surface}

The friction force that occurs in the contact is a combination of different forces caused by different mechanisms adhesion and deformation, which gives the so-called two-term model

$$
F_{\mu}=F_{\mu, a d h}+F_{\mu, d e f}
$$

The adhesion component in the friction force can be expressed as the product of the shear strength of the interface and the real area of the contact. When an asperity that indents the skin is moved forward, energy is dissipated due to the viscoelastic nature of the skin. The deformation component equals the amount of work lost by viscoelastic hysteresis per unit sliding distance, thus being proportional to the indentation depth of the asperities into the skin. In an earlier paper, the authors investigated the friction behaviour of the human fingerpad as a function of asperity geometry [27]. In vivo experiments were carried out sliding the fingerpad of a single subject against regular surface textures. Using analytical expressions, a multiscale model was developed to explain the observed friction behaviour as a function of asperity density and geometry, normal load and skin condition. The contact of the fingerpad with a nominally flat, rigid surface is described by considering the fingerpad

as a deformable sphere, which gives a relation between the apparent contact area $A_{0}$ and the applied load $F$ following

$$
A_{0} \propto F_{n}^{\alpha}
$$

Due to the layered structure of the skin and the support by the stiffer bone of the distal phalanx, the mechanical response of the fingerpad, and thus the magnitude of the apparent contact area, changes with applied load. Eq. (5) shows that the growth of the apparent contact area with applied load follows a power law. Exponents range between 0.2 and 0.55 $[29,30,35]$. For the index finger of the subject used in the current study an exponent $\alpha=0.36$ was obtained experimentally by measuring the contact area (using an inkpad) 
for various values of the applied normal load. [27].

In a next step, the fingerprint ridges are modelled as annulus-shaped line contacts, characterized by a width $2 a_{1}$ and the number of ridges per unit length $\eta$, so that for the fingerprint area $A_{1}$

$$
A_{1} \propto 2 a_{1} \cdot \eta \cdot A_{0}
$$

For the index finger of the subject used in the current study for $2 a_{1} \eta$ a value of 0.58 was measured from a silicone replica of the fingerpad skin [27]. When in contact with a surface texture, which is composed of spherical-tipped asperities, the real area of contact is determined by the contact behaviour at the micro scale. In this case, the friction force is calculated by the real area of contact and the indentation of the asperities at the micro scale. Since the contact between the asperities and the fingerprint ridges is formed by the stratum corneum, the top layer of the skin, the size of the real contact area is determined by the properties of this top layer. The most important characteristic of this layer is the dependence of its properties on the hydration level as discussed earlier. Furthermore, since untreated human skin is covered with a mixture of water and sebum, generally referred to as a lipid film, adhesive forces between the asperities and the skin should be taken into account. Pailler-Mattéi and Zahouani [36] measured normal adhesion forces from indentation experiments on in vivo skin. Experiments were carried out on normal, i.e. untreated, and dry, i.e. treated with ether, skin of the inner forearm. Results showed that a thin surface film on the skin is responsible for the adhesive behaviour and that the magnitude of the adhesion forces changed considerably with skin condition. Although the glabrous skin of the hand does not contain any sebaceous glands, it does have a high density of sweat ducts supplying the skin of the fingerpad with a small amount of sweat to enhance grip by increasing adhesion. So, in contrast to the apparent fingerprint area, which is determined by the applied load or pressing force, the real contact area is determined by an apparent normal load $F_{a p p}$ which is a combination of the applied load and adhesive forces between the skin and the contacting surface. For a regular texture such as used in the experiments, the number of asperities in contact with the skin $N$ is determined by the spacing between the asperities $\lambda$ and can be obtained from the fingerprint area $A_{1}=N \cdot \lambda^{2}$. From the contact area between an individual asperity with tip radius $R$ and the skin, the real contact area can now be calculated. For simplicity the mechanical behaviour of the top layer of the skin is assumed to be constant so that straightforward use of available contact mechanics theory [37] leads to the following relations for real contact area $A_{\text {real }}$ and relative indentation $\delta / a$ :

$$
A_{\text {real }} \propto F_{a p p}^{2 / 3} \cdot A_{0}^{1 / 3} \cdot R^{2 / 3} \cdot \lambda^{-2 / 3}
$$




$$
\frac{\delta}{a} \propto F_{a p p}^{1 / 3} \cdot A_{0}^{-1 / 3} \cdot R^{-2 / 3} \cdot \lambda^{2 / 3}
$$

Equations (7) and (8) describe the relation between respectively adhesive and deformation friction and the texture characteristics tip radius $R$ and spacing $\lambda$, thus guiding the optimization of surface textures with respect to friction behaviour.

\subsection{Experiments using textured surfaces}

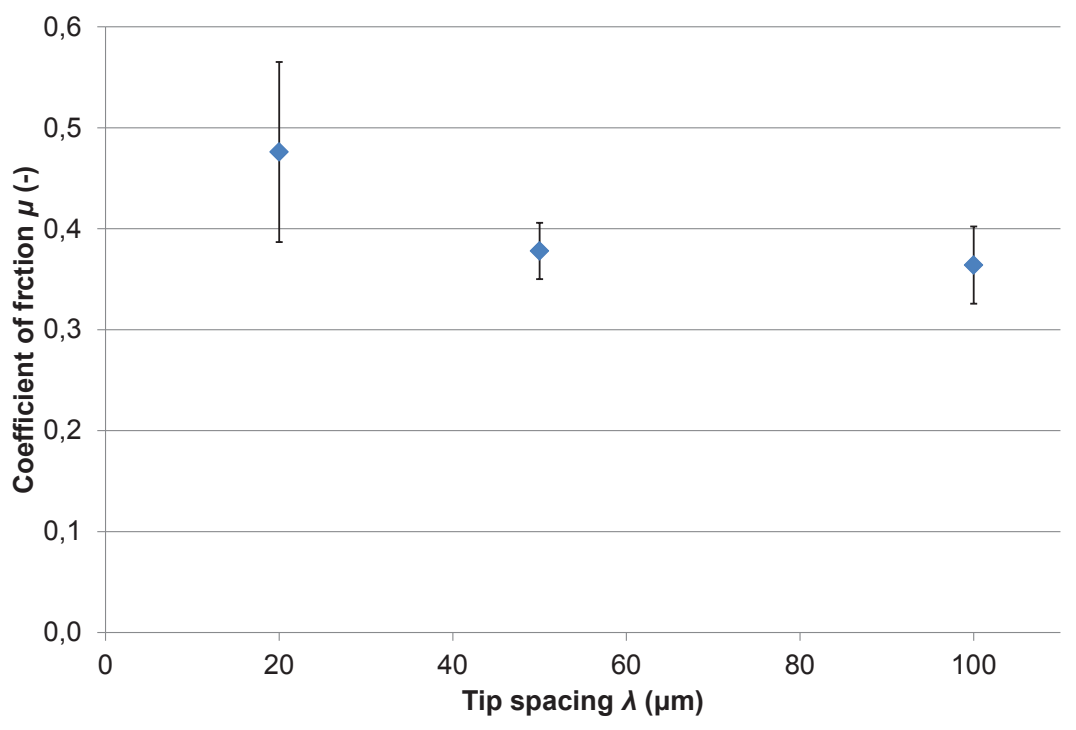

Figure 11. Coefficient of friction as a function of tip spacing for the normal skin of the index finger at $1 \mathrm{~N}$ normal load and $\approx 30^{\circ}$ orientation: mean values and standard deviations.

Fig. 11 shows the coefficient of friction measured against textured samples as a function of tip spacing and normal load. With the tip spacing increasing from 20 to 50 and $100 \mu \mathrm{m}$, at $1 \mathrm{~N}$ normal load the coefficient of friction, averaged over five measurements, decreases from 0.48 to 0.38 and 0.36 respectively. Standard deviations vary between $8 \%$ and $20 \%$. Fig. 12 shows the coefficient of friction measured against textured samples as a function of tip radius of curvature and normal load. At $1 \mathrm{~N}$ normal load and $20 \mathrm{\mu m}$ tip spacing the average coefficient of friction increases from 0.4 to 0.48 when the tip radius increases from 2 to $5 \mu \mathrm{m}$. At $100 \mu \mathrm{m}$ tip spacing the average coefficient of friction remains constant at 0.36 when the tip radius increases from 5 to $10 \mu \mathrm{m}$. 


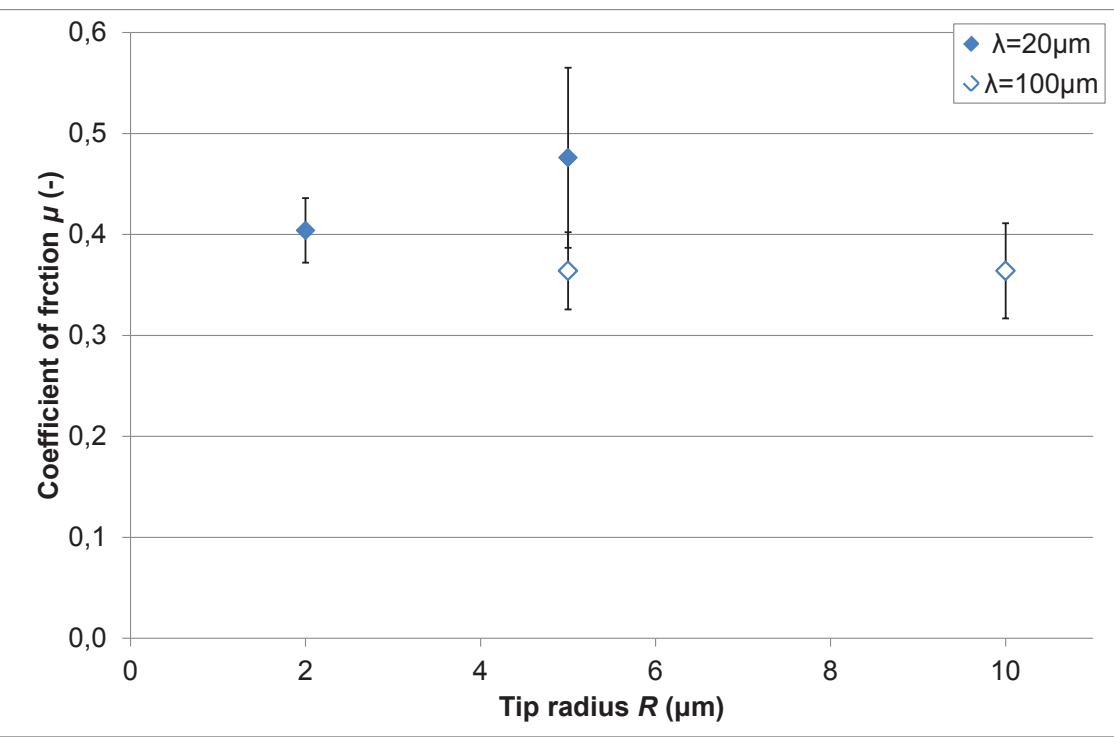

Figure 12. Coefficient of friction as a function of tip radius for the normal skin of the index finger at $1 \mathrm{~N}$ normal load and $\approx 30^{\circ}$ orientation: mean values and standard deviations

\subsection{The role of normal adhesion}

Fig. 11 shows that the coefficient of friction decreases with increasing tip spacing. Since this corresponds to the trend predicted by Eq. (7), it can be concluded that adhesive friction is the dominant mechanism especially at small spacing. At larger spacing the deformation component of friction becomes more important so that the trend of a decreasing coefficient of friction becomes weaker. Adhesive friction being the dominant friction mechanism suggests a clear dependence of the coefficient of friction on tip radius as described by Eq. (7). Fig. 12 shows that for normal skin at a tip spacing of $20 \mu \mathrm{m}$ an increase of the tip radius from 2 to $5 \mu \mathrm{m}$ leads to an increase of the coefficient of friction. At a tip spacing of $100 \mu \mathrm{m}$, an increase of the tip radius from 5 to $10 \mu \mathrm{m}$ appears to have no effect on the average value of the measured coefficient of friction.

Since the number of contacts is high when the distance between the tips is small, the applied load on each individual asperity is low. Therefore, the normal adhesion between the skin and the countersurface contributes considerably to the actual load. The contribution of normal adhesion increases with increasing tip radius, hence the higher coefficient of friction. Following the theory proposed in [27], different mechanisms determine the magnitude of the coefficient of friction for these regular surface geometries. At larger tip spacings the contribution of normal adhesion is not as important as it is at smaller tip spacings. Due to the larger tip spacing the deformation component of friction comes into play. The effect of tip radius on the different mechanisms adhesive and deformation friction is reversed as 
shown in Eqs. (7) and (8). The relative contributions of the different friction mechanisms are sensitive to differences in test conditions such as the state of the skin.

From Figs. 11 and 12 it appears that the variation in results is the largest when, following the theory developed earlier, adhesion has the most influence. It is common to describe the frictional properties of a surface by the coefficient of friction which is obtained by dividing the measured friction force by the applied normal load following Eq. (1). However, what is measured is the friction force which results from the apparent normal load, so that actually

$$
\begin{aligned}
F_{\mu} & =\mu_{\text {real }} \cdot F_{\text {real }} \\
& =\mu_{\text {real }} \cdot\left(F_{n}+F_{a d h}\right)
\end{aligned}
$$

When the measured coefficient of friction $\mu$ is calculated by using Eq. (1), what is actually calculated is

$$
\mu=\mu_{\text {real }} \cdot\left(1+\frac{F_{a d h}}{F_{n}}\right)
$$

Eq. (10) shows that the effect of adhesion becomes important when the normal load is of the same order of magnitude as the adhesion force. For example for a textured sample with $5 \mu \mathrm{m}$ radius asperities at $20 \mu \mathrm{m}$ spacing, at $1 \mathrm{~N}$ normal load the total adhesion force $F_{a d h}$ varies between 0 and $0.3 \mathrm{~N}$, depending on the surface condition of the skin [27]. So under conditions where adhesion plays a role, variations in hydration level and cleanliness of the skin will result in considerable variations in the adhesion force and thus in variations

in the observed friction. This is confirmed by the very poor repeatability of results of measurements carried out against smooth samples where there is no deformation. The thin lipid layer which may be present on the skin could increase the friction through its adhesive properties. However, this same lipid layer might also act as a lubricating layer on the interface, which against a smooth counter surface forms a friction reducing layer.

\subsection{Experiments using sandpaper}

Fig. 13 shows the coefficient of friction between the fingerpad and sandpaper for normal and damp skin. For normal skin friction increases with increasing particle diameter from an approximate coefficient of friction of 0.93 at a diameter of $10.3 \mu$ to 1.48 at a particle diameter of $201 \mu$. Variation is relatively small with standard deviations between 2 and $10 \%(n=5)$. For damp skin the coefficient of friction decreases with increasing particle diameter from about 1.62 at $10.3 \mu \mathrm{m}$ to 1.15 at $201 \mu \mathrm{m}$ with standard deviations varying between 4 and $14 \%$. 


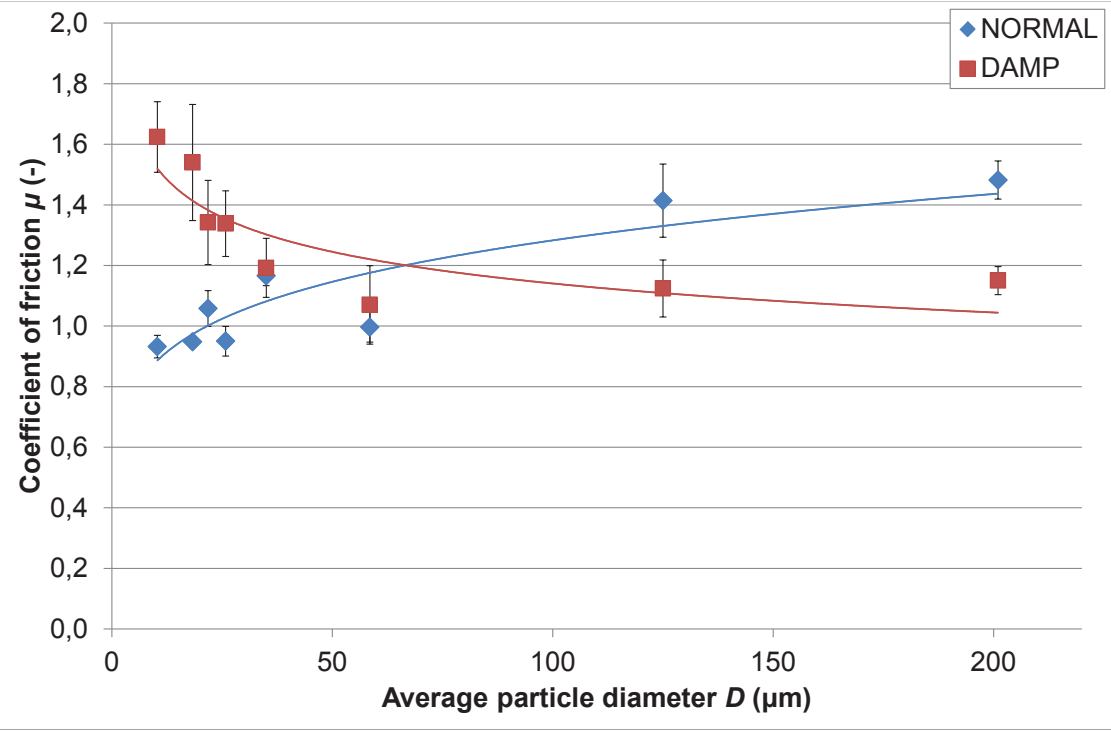

Figure 13. Coefficient of friction measured against sandpaper as a function of particle diameter for normal and damp skin of the index finger at $1 \mathrm{~N}$ normal load and $\tilde{3} 0^{\circ}$ orientation: mean values and standard deviations

Several authors found that friction decreases with roughness amplitude $[3,13,14,17$, 38]. From a certain point, when the roughness amplitude increases further, increased ploughing leads to an increase of the friction as reported by Tomlinson et al. [16]. Although the observed trends in the different studies are consistent, it is the geometry of the surface rather than the surface roughness amplitude that determines the friction behaviour. With increasing particle diameter the density of the abrasive particles decreases, which increases the load on each individual particle. It was shown earlier that when the load on an individual asperity increases, deformation becomes the more important friction mechanism. Increased ploughing explains the increase of the coefficient of friction with particle diameter.

\subsection{The effect of skin hydration}

The decreasing friction observed with damp skin is caused by the dependence of the mechanical properties on the hydration level of the stratum corneum, the top layer of the skin. Softening of the stratum corneum leads to an increased contact area and increased indentation depth. The adhesive component of the friction force increases, especially at small particle diameters, where the density of the particles is high. Whereas for normal skin the deformation component is negligible at small particle diameters, for damp skin deformation friction does play a role. 
Although Fig. 13 plots the coefficient of friction as a function of particle diameter, friction is also determined by other factors, such as the shape and the particle density. Since these properties do not necessarily correlate, the relation between particle diameter and the coefficient of friction plotted in Fig. 13 does not follow a smooth line.

\section{$5 \quad$ Summarizing conclusions}

It can be concluded that the friction behaviour one observes when exploring a surface by touching is susceptible to different sources of variation which are intrinsic to tactile exploration. The variation of the observed friction can be assigned to differences in contacting area which are caused by a combination of orientation of the finger and pressing force. Furthermore, the mechanical response of the skin, which determines the real contact area, changes with the hydration level of the skin.

Under conditions where normal adhesion plays an important role, such as with smooth surfaces or textures with high asperity densities, the existing method of measuring friction of the fingerpad appears not to be not very robust. Adhesion forces, and thus measured friction, change with hydration level and cleanliness of the skin leading to a large variation in observed results.

Since in general tactile exploration involves contact with more fingers as well as different types of motion, the correlation between slipperiness and the coefficient of friction measured in a more or less controlled test environment is no sinecure. Therefore, when carrying out each friction measurement a well-defined experimental procedure should be followed, which is described in the following section.

\section{How to measure tactile friction in vivo}

The principle of a stationary sample over which the subjects slide their fingerpads is a proven method for measuring tactile friction. To assure high levels of repeatability, the samples should be thoroughly cleaned. Soap washing and rinsing with water for the skin of the fingerpad and ultrasonic cleaning in ethanol for the stationary samples suffice. The measurements should be carried out under constant environmental conditions.

Considerable differences might exist in the frictional properties of the different fingers. When the objective is to relate the measured friction properties to the perception of slipperiness or smoothness, which is mostly determined by multifinger contact, it is recommended to measure the frictional behaviour of all the fingers involved in tactile exploration.

The friction measurement should comprise the measurement of the tangential friction force as well as the normal load, or pressing force, on the contact. For low loads, in the 
order of several Newtons, the use of dead weights attached to the fingerpad is preferred to aid the application of a more or less constant load by the subject. Furthermore, the hydration level of the skin should be monitored during the test program. Specifically, when more samples with the same properties are being used, it is highly recommended to measure the surface topography of all samples to check for unexpected differences in geometry of the surface.

When analyzing the measurement results it is important to acknowledge that although the measured coefficient of friction is obtained by dividing the measured friction force by the applied normal load, different mechanisms lie behind this single parameter. Variations in the contact area between the fingerpad and the sample can be caused by the orientation angle and pressing force. For surfaces where normal adhesion becomes important, repeatability of the results will get worse, since changes in skin condition lead to considerable changes in the measured coefficient of friction.

Considering the human aspects of the tactile friction measurement will enable researchers to draw the correct conclusions on the relation between physical properties, frictional behaviour and perceived qualities of the surface.

\section{Acknowledgements}

This research was carried out under project number M63.2.10409 in the framework of the Research Program of the Materials innovation institute M2i (www.m2i.nl).

\section{References}

1. C.J. Barnes, T.H.C. Childs, B. Henson, and C.H. Southee. Surface finish and touch a case study in a new human factors tribology. Wear, 257(7-8):740-750, 2004.

2. T. H. C. Childs and B. Henson. Human tactile perception of screen-printed surfaces: Self-report and contact mechanics experiments. Proceedings of the Institution of $\mathrm{Me}$ chanical Engineers, Part J: Journal of Engineering Tribology, 221(3):427-441, 2007.

3. X. Liu, Z. Yue, Z. Cai, D. G. Chetwynd, and S. T. Smith. Quantifying touch-feel perception: tribological aspects. Measurement Science and Technology, 19(8):084007, 2008.

4. X. Chen, C. J. Barnes, T. H. C. Childs, B. Henson, and F. Shao. Materials' tactile testing and characterisation for consumer products' affective packaging design. Materials \& Design, 30(10):4299-4310, 2009. 
5. Matthew A. Darden and Christian J. Schwartz. Investigation of skin tribology and its effects on the tactile attributes of polymer fabrics. Wear, 267(5-8):1289-1294, 2009.

6. Lisa Skedung, Katrin Danerl ov, Ulf Olofsson, Carl Michael Johannesson, Maiju Aikala, John Kettle, Martin Arvidsson, Birgitta Berglund, and Mark W. Rutland. Tactile perception: Finger friction, surface roughness and perceived coarseness. Tribology International, 44(5):505-512, 2011.

7. Sabrina Schreiner, Marcus Rechberger, and Jürgen Bertling. Haptic perception of friction - correlating friction measurements of skin against polymer surfaces with subjective evaluations of the surfaces' grip. Tribology International, 63:21-28, 2013.

8. Wouter M. Bergmann Tiest. Tactual perception of material properties. Vision Research, 50(24):2775-2782, 2010.

9. M. M. Taylor and S. J. Lederman. Tactile roughness of grooved surfaces: A model and the effect of friction. Perception \& Psychophysics, 17(1):23-36, 1975.

10. A. R. Gwosdow, J. C. Stevens, L. G. Berglund, and J. A. J. Stolwijk. Skin friction and fabric sensations in neutral and warm environments. Textile Research Journal, 56(9):574-580, 1986.

11. Allan M. Smith, C. Elaine Chapman, Mélanie Deslandes, Jean-Sébastien Langlais, and Marie-Pierre Thibodeau. Role of friction and tangential force variation in the subjective scaling of tactile roughness. Experimental brain research, 144(2):211-223, 2002.

12. S. Derler and L.-C. Gerhardt. Tribology of skin: Review and analysis of experimental results for the friction coefficient of human skin. Tribology Letters, 45:1-27, 2011.

13. O. S. Dinç, C. M. Ettles, S. J. Calabrese, and H. A. Scarton. Some parameters affecting tactile friction. Journal of Tribology, 113(3):512-517, 1991.

14. M.G. Gee, P. Tomlins, A. Calver, R.H. Darling, and M. Rides. A new friction measurement system for the frictional component of touch. Wear, 259(7-12):1437-1442, 2005.

15. R. Lewis, C. Menardi, A. Yoxall, and J. Langley. Finger friction: Grip and opening packaging. Wear, 263(7-12):1124-1132, 2007.

16. S.E. Tomlinson, R. Lewis, and M.J. Carré. The effect of normal force and roughness on friction in human finger contact. Wear, 267(5-8):1311-1318, 2009. 
17. M. A. Masen. A systems based experimental approach to tactile friction. Journal of the Mechanical Behavior of Biomedical Materials, 4:1620-1626, 2011.

18. S. Derler, J. Süess, A. Rao, and G.-M. Rotaru. Influence of variations in the pressure distribution on the friction of the finger pad. Tribology International, pages 14-20, 2013.

19. N. K. Veijgen, M. A. Masen, and E. van der Heide. Relating friction on the human skin to the hydration and temperature of the skin. Tribology Letters, 49(1):251-262, 2013.

20. R. T. Verrillo, S. J. Bolanowski, C. M. Checkosky, and F. P. McGlone. Effects of hydration on tactile sensation. Somatosensory $\& 3$ Motor Research, 15(2):93-108, 1998.

21. Anastasia B Cua, K. P. Wilhelm, and H. I. Maibach. Frictional properties of human skin: relation to age, sex and anatomical region, stratum corneum hydration and transepidermal water loss. British Journal of Dermatology, 123(4):473-479, 1990.

22. L.-C Gerhardt, V Strässle, A Lenz, N. D. Spencer, and S Derler. Influence of epidermal hydration on the friction of human skin against textiles. Journal of The Royal Society Interface, 5(28):1317-1328, 2008.

23. M. Kwiatkowska, S. E. Franklin, C. P. Hendriks, and K. Kwiatkowski. Friction and deformation behaviour of human skin. Wear, 267(5-8):1264-1273, 2009.

24. Yonghui Yuan and Ritu Verma. Measuring microelastic properties of stratum corneum. Colloids and Surfaces B: Biointerfaces, 48(1):6-12, 2006.

25. C. Pailler-Mattéi, S. Pavan, R. Vargiolu, F. Pirot, F. Falson, and H. Zahouani. Contribution of stratum corneum in determining bio-tribological properties of the human skin. Wear, 263(7-12):1038-1043, 2007.

26. J. Van Kuilenburg, M.A. Masen, and E. Van der Heide. Contact modelling of human skin: What value to use for the modulus of elasticity? Proceedings of the Institution of Mechanical Engineers, Part J: Journal of Engineering Tribology, 227(4):347-359, 2013.

27. J. Van Kuilenburg, M. A. Masen, and E. Van der Heide. The role of the skin microrelief in the contact behaviour of human skin: Contact between the human fingertip and regular surface textures. submitted to Tribology International. 
28. S. S. Stevens and Judith Rich Harris. The scaling of subjective roughness and smoothness. Journal of Experimental Psychology, 64(5):489-494, 1962.

29. P. H. Warman and A. R. Ennos. Fingerprints are unlikely to increase the friction of primate fingerpads. Journal of Experimental Biology, 212(13):2016-2022, 2009.

30. Makoto Tomimoto. The frictional pattern of tactile sensations in anthropomorphic fingertip. Tribology International, 44(11):1340-1347, 2011.

31. Ramona Fagiani, Francesco Massi, Eric Chatelet, Jean Philippe Costes, and Yves Berthier. Contact of a finger on rigid surfaces and textiles: Friction coefficient and induced vibrations. Tribology Letters, 48(2):145-158, 2012.

32. Thibaut André, Philippe Lefévre, and Jean-Louis Thonnard. A continuous measure of fingertip friction during precision grip. Journal of Neuroscience Methods, 179(2):224$229,2009$.

33. Lisa Skedung, Katrin Danerlöv, Ulf Olofsson, Maiju Aikala, Kari Niemi, John Kettle, and Mark W. Rutland. Finger friction measurements on coated and uncoated printing papers. Tribology Letters, 37(2):389-399, 2010.

34. R. T. Spurr. Fingertip friction. Wear, 39(1):167-171, 1976.

35. S. Derler, L.-C. Gerhardt, A. Lenz, E. Bertaux, and M. Hadad. Friction of human skin against smooth and rough glass as a function of the contact pressure. Tribology International, 42(11-12):1565-1574, 2009.

36. C. Pailler-Mattéi and H. Zahouani. Analysis of adhesive behaviour of human skin in vivo by an indentation test. Tribology International, 39(1):12-21, 2006.

37. K. L. Johnson. Contact mechanics. Cambridge University Press, Cambridge, 1999.

38. T. Sasada. The friction of human skin. In Proceedings of the 21st Biotribology Symposium, JSME, Fukuoka, 2000, pages 46-52, 2000. 


\section{Paper F}

\section{A mechanistic approach to predicting the friction behaviour of human skin}

J. van Kuilenburg, M.A. Masen and E. van der Heide

Proceedings of the ASME/STLE 2012 International Joint Tribology Conference

Denver, USA, 2012

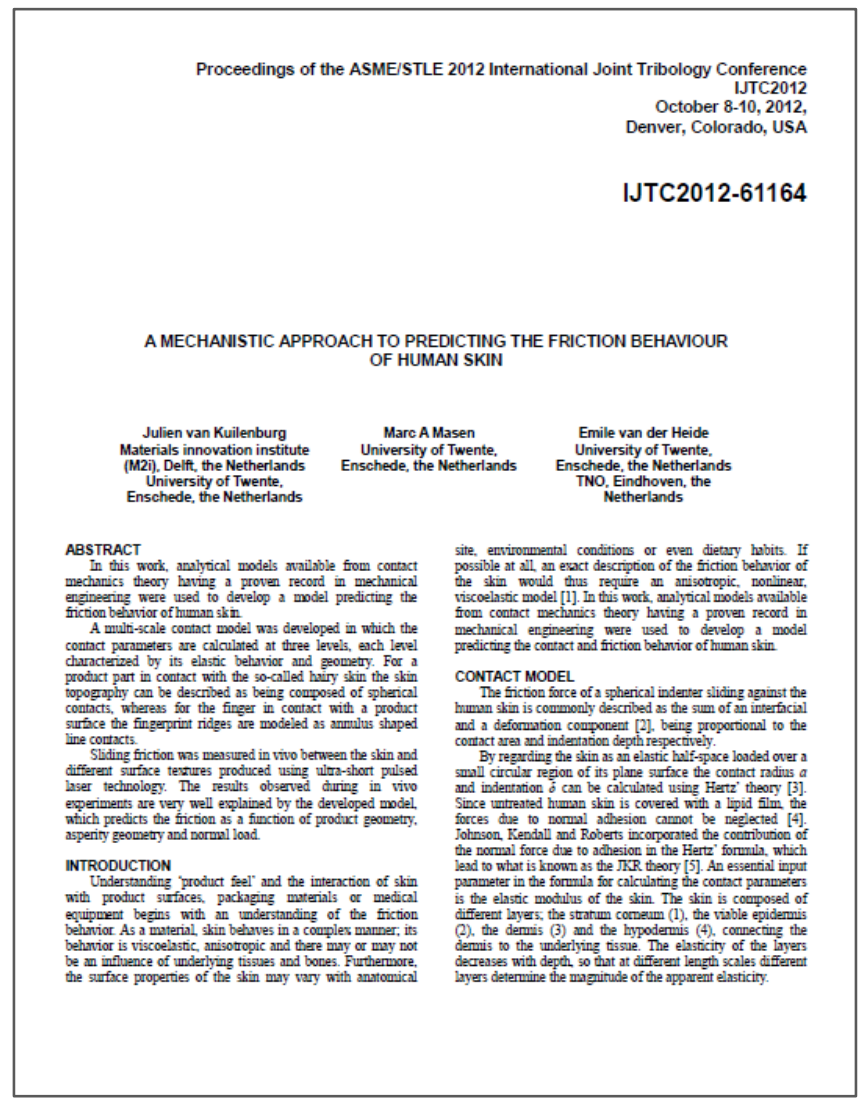





\title{
A MECHANISTIC APPROACH TO PREDICTING THE FRICTION BEHAVIOUR OF HUMAN SKIN
}

\author{
Julien van Kuilenburg, Marc A. Masen and Emile van der Heide
}

\begin{abstract}
In this work, analytical models available from contact mechanics theory having a proven record in mechanical engineering were used to develop a model predicting the friction behavior of human skin.

A multi-scale contact model was developed in which the contact parameters are calculated at three levels, each level characterized by its elastic behaviour and geometry. For a product part in contact with the so-called hairy skin the skin topography can be described as being composed of spherical contacts, whereas for the finger in contact with a product surface the fingerprint ridges are modelled as annulus-shaped line contacts.

Sliding friction was measured in vivo between the skin and different surface textures produced using ultra-short pulsed laser technology. The results observed during in vivo experiments are very well explained by the developed model, which predicts the friction as a function of product geometry, asperity geometry and normal load.
\end{abstract}

\section{INTRODUCTION}

Understanding 'product feel'and the interaction of skin with product surfaces, packaging materials or medical equipment begins with an understanding of the friction behaviour. As a material, skin behaves in a complex manner; its behaviour is viscoelastic, anisotropic and there may or may not be an influence of underlying tissues and bones. Furthermore, the surface properties of the skin may vary with anatomical site, environmental conditions or even dietary habits. If possible at all, an exact description of the friction behaviour of the skin would thus require an anisotropic, nonlinear, viscoelastic model [1]. In this work, analytical models available from contact mechanics theory having a proven record in mechanical engineering were used to develop a model predicting the contact and friction behaviour of human skin. 


\section{CONTACT MODEL}

The friction force of a spherical indenter sliding against the human skin is commonly described as the sum of an interfacial and a deformation component [2], being proportional to the contact area and indentation depth respectively. By regarding the skin as an elastic half-space loaded over a small circular region of its plane surface the contact radius $a$ and indentation $\delta$ can be calculated using Hertz's theory [3]. Since untreated human skin is covered with a lipid film, the forces due to normal adhesion cannot be neglected [4]. Johnson, Kendall and Roberts incorporated the contribution of the normal force due to adhesion in the Hertz formula, which led to what is known as the JKR theory [5]. An essential input parameter in the formula for calculating the contact parameters is the elastic modulus of the skin. The skin is composed of different layers; the stratum corneum (1), the viable epidermis (2), the dermis (3) and the hypodermis (4), connecting the dermis to the underlying tissue. The elasticity of the layers decreases with depth, so that at different length scales different layers determine the magnitude of the apparent elasticity.

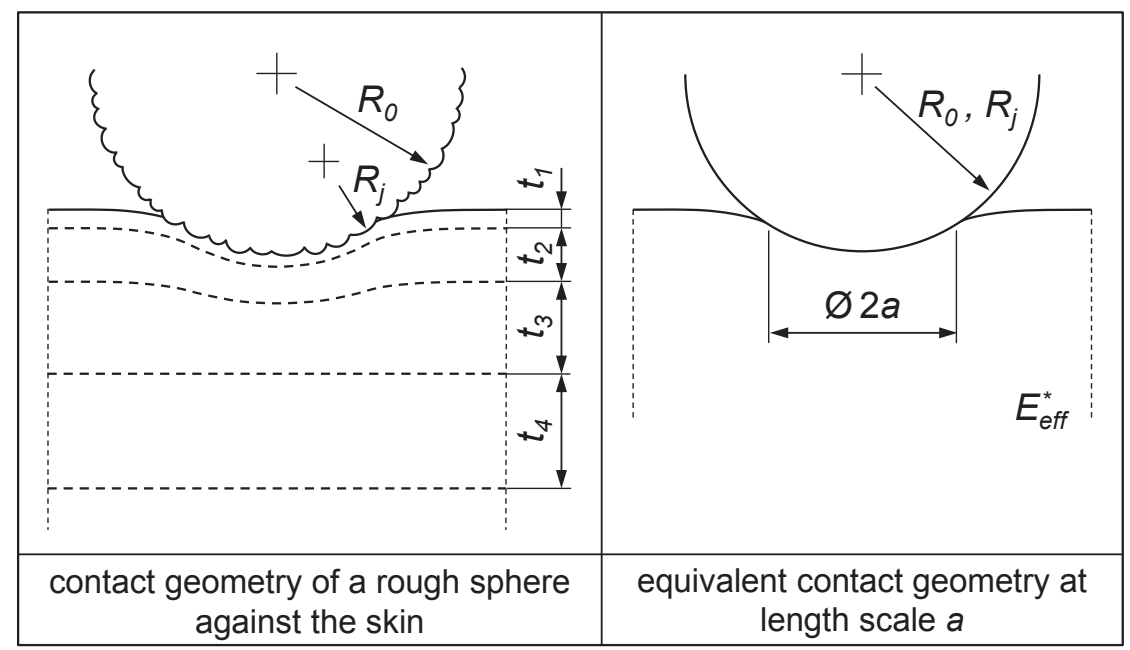

Figure 1. Schematic illustration of the concept of the effective elastic modulus

\section{Elastic properties}

To account for the multilayered and nonhomogeneous structure of the skin, the concept of an effective elastic modulus was adopted, which led to a closed-form expression $E=f(a)$ describing the elasticity of the skin as a function of length scale [6]. This is depicted schematically in Fig. 1. In the case of a spherical contact the length scale of the contact equals the contact radius $a$. Figure 2 shows that the effective elastic modulus of the volar 
forearm varies considerably with length scale. At different scales the evolution of the effective elastic modulus with contact length scale can be described using a power law, which enables substitution into the available contact mechanics formula.



Figure 2. Effective elastic modulus of the skin of the volar forearm as a function of length scale. Adapted from $[6]$

\section{Surface topography}

To incorporate the influence of the skin microrelief a multiscale contact model was developed in which the surface topography of the skin is considered as a nominal flat surface having a bi-sinusoidal profile characterized by a wavelength and amplitude [7]. An average radius of curvature $R_{i}$ was calculated after measuring surface feature density and roughness amplitude $R_{z}$. For a smooth spherical probe in contact with the skin of the volar forearm this simplified geometry is illustrated in Fig. 3. The contact areas of the individual surface features are calculated using the theory proposed by Archard [8]; as a result of the Hertzian pressure distribution the area of the contact spots in the centre of the macro contact is considerably larger than the area of the contact spots at the edge.

\section{Friction}

The friction force is calculated from the real contact area and the indentation at the macro scale. The evolution of the friction force $F$ with normal load $F$ was calculated for nine 


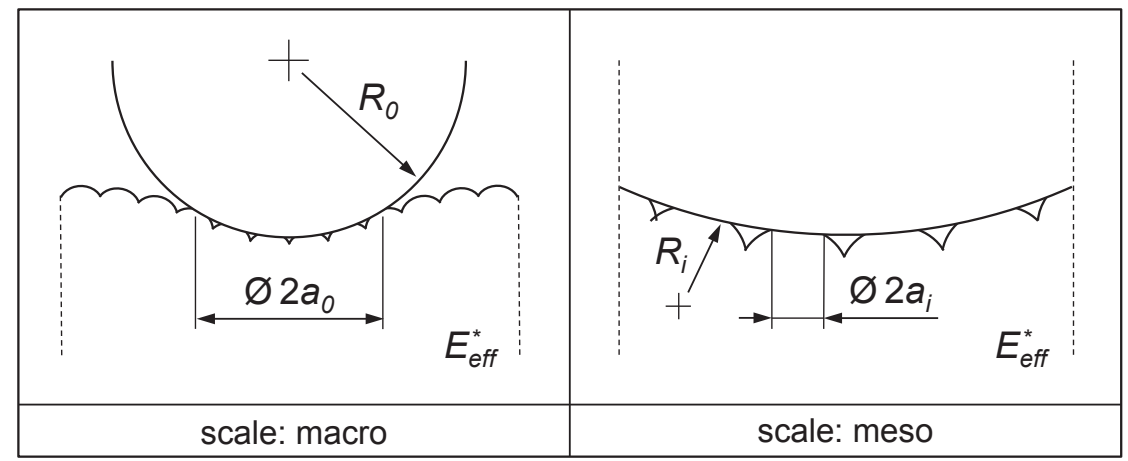

Figure 3. Contact dimensions of a smooth spherical probe in contact with the skin of the volar forearm.

different cases reported in the literature using the two-term friction model [2]:

$$
F_{\mu}=F_{\mu, a d h}+F_{\mu, d e f} \approx k F^{n}
$$

From analysis of the different studies it was found that the power $n$ varies with experimental conditions [9]. For cleaned skin $n$ is larger than 1 . For untreated skin $n$ is smaller than 1 due to the increasing contribution of normal adhesion, and for wet skin eventually. Comparison of calculated and reported values for $\mathrm{n}$ shows good agreement as shown in Fig. 4.

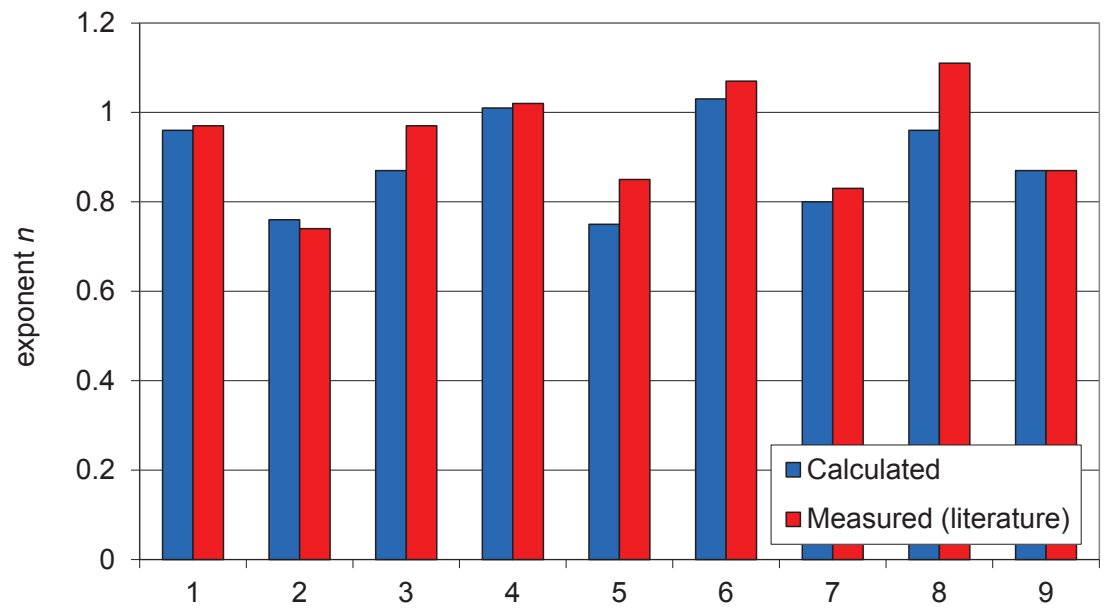

Figure 4. Comparison of model predictions with experimental results collected from literature. Adapted from [9]. 


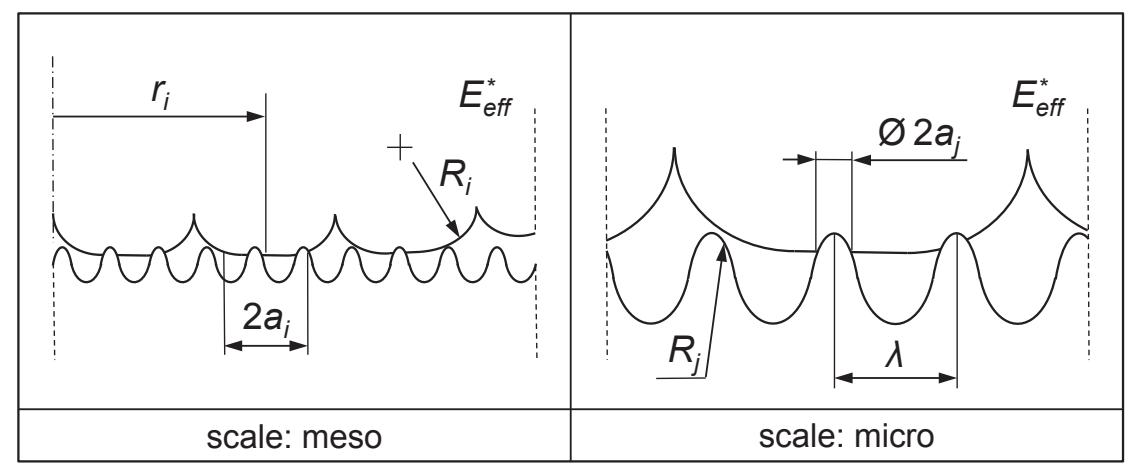

Figure 5. Contact dimensions of the fingertip ridges in contact with a regular surface texture.

\section{TEXTURED SURFACES}

To investigate the friction behaviour at the asperity-level, surface textures consisting of evenly distributed spherically-tipped asperities were produced using ultra-short pulsed laser technology (www.lightmotif.nl). The texture parameters tip radius $R$ and spacing $\lambda$ varied between 2 and $10 \mu \mathrm{m}$ and 20 and $100 \mu \mathrm{m}$ respectively. Sliding friction was measured in vivo between the skin of the fingerpad and textured metal samples measuring $20 \times 40$ $\mathrm{mm}^{2}$ using a 6 DOF load cell (ATI Gamma, www.ati-ia.com). The surface topography of the fingerpad was measured from a silicone replica using a $3 \mathrm{D}$ optical profilometer (VK9700, www.keyence.com). Hydration level of the skin and thickness of the lipid layer were monitored during the experiments using a Corneometer CM825 and Sebumeter SM815 (www.courage-khazaka.de). The contact of the fingerpad with a nominally flat, rigid surface can be described analogous to the smooth spherical probe in contact with the flat skin by considering the fingerpad as a deformable sphere. The fingerprint ridges are modelled as annulus-shaped line contacts, characterized by a radius $r_{i}$ and contact width $2 a_{i}$. When in contact with a surface texture which is composed of spherically-tipped asperities, the real area of contact is determined by the contact behaviour at the micro scale as depicted schematically in Fig. 5. In this case the friction force is calculated from the real area of contact and the indentation of the asperities at the micro scale. Figure 6 shows the results obtained from experiments (dots). The calculated coefficient of friction is plotted as a function of tip radius $R$ and spacing between asperities $\lambda$ (lines). The coefficient of friction increases with increasing tip radius. This effect can be attributed to the increasing contribution of normal adhesion, which is confirmed by JKR theory. At constant tip radius the coefficient of friction shows a strong decrease with increasing spacing. At larger spacing the coefficient of friction tends to increase with increasing spacing. The two-term 
friction model shows that at small spacing the friction force arises mainly from interfacial shear, which decreases with increasing spacing, whereas at large spacing the deformation component cannot be neglected anymore.

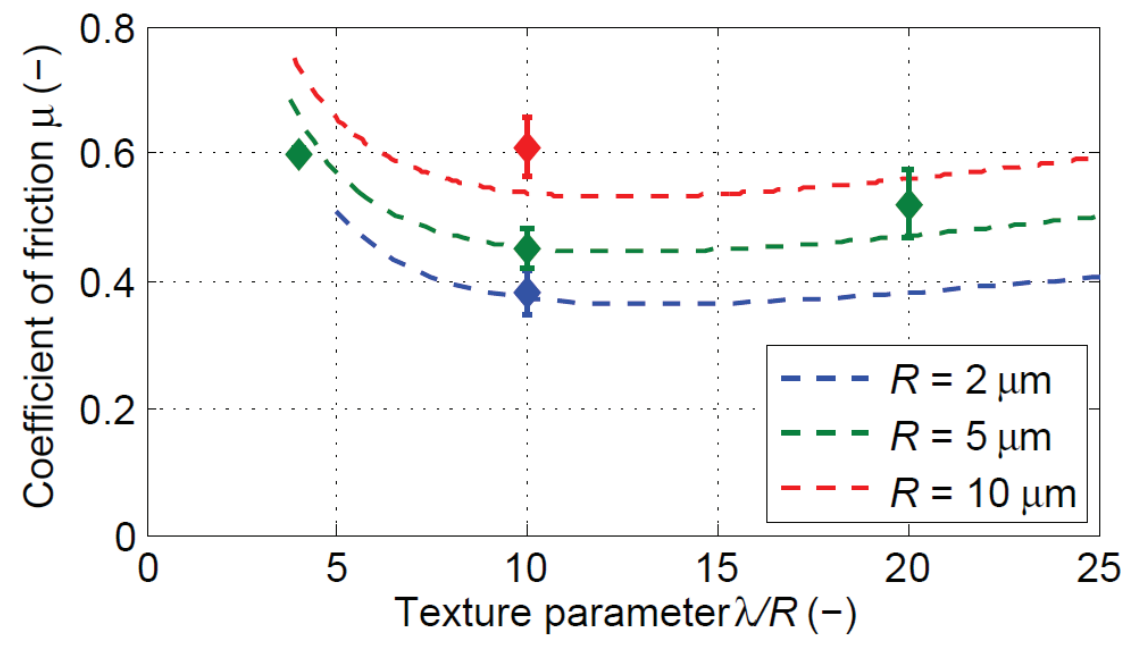

Figure 6. Coefficient of friction of the fingerpad sliding against a surface texture as a function of tip radius $R$ and spacing $\lambda$ : experimental results (dots) and modelling results (lines).

\section{CONCLUSIONS}

The results observed during in vivo experiments are very well explained by the developed contact and friction model, which predicts the friction as a function of product geometry, asperity geometry and normal load. Further work will involve the definition of design rules for the engineering of surfaces in contact with the skin, which are based on the friction model.

\section{ACKNOWLEDGEMENTS}

This research was carried out under the project number M63.2.10409 in the framework of the Research Programme of the Materials innovation institute M2i (www.m2i.nl). 


\section{REFERENCES}

1. S. Derler and L.-C. Gerhardt. Tribology of skin: Review and analysis of experimental results for the friction coefficient of human skin. Tribology Letters, 45:1-27, 2011.

2. M.J. Adams, B.J. Briscoe, and S.A. Johnson. Friction and lubrication of human skin. Tribology Letters, 26(3):239-253, 2007.

3. H. Hertz. Über die Berührung fester elastischer Körper. Journal für die reine und angewandte Mathematik, 92:156-171, 1882.

4. C. Pailler-Mattéi and H. Zahouani. Analysis of adhesive behaviour of human skin in vivo by an indentation test. Tribology International, 39(1):12-21, 2006.

5. K.L. Johnson, K. Kendall, and A.D. Roberts. Surface energy and the contact of elastic solids. Proceedings of the Royal Society of London. A. Mathematical and Physical Sciences, 324(1558):301-313, 1971.

6. J. Van Kuilenburg, M. A. Masen, and E. Van der Heide. Contact modelling of human skin: What value to use for the modulus of elasticity? submitted to the Proceedings of the Institution of Mechanical Engineers, Part J: Journal of Engineering Tribology.

7. K.L. Johnson, J.A. Greenwood, and J.G. Higginson. The contact of elastic regular wavy surfaces. International Journal of Mechanical Sciences, 27(6):383-396, 1985.

8. J.F. Archard. Elastic deformation and the laws of friction. Proceedings of the Royal Society of London. Series A. Mathematical and Physical Sciences, 243(1233):190-205, 1957.

9. J. Van Kuilenburg, M. A. Masen, and E. Van der Heide. The role of the skin microrelief in the contact behaviour of human skin: Contact between a spherical probe and human skin. submitted to Tribology International. 
